\title{
Transversals of graphs
}

\author{
Juan Gutiérrez
}

TESE APRESENTADA

AO

Instituto de Matemática E Estatística

DA

Universidade de São Paulo

PARA

OBTENÇÃO DO TÍTULO

DE

DOUTOR EM CIÊNCIAS

\section{Programa: Doutorado em Ciência da Computação Orientadora: Profa. Dra. Cristina Gomes Fernandes}

Durante o desenvolvimento deste trabalho o autor recebeu auxílio financeiro da

Fundação de Amparo à Pesquisa do Estado de São Paulo (FAPESP) 1

São Paulo, February 25, 2019

\footnotetext{
${ }^{1}$ Processo 2015/08538-5. As opiniões, hipóteses e conclusões ou recomendações expressas neste material são de responsabilidade do autor(es) e não necessariamente refletem a visão da FAPESP.
} 


\section{Transversals of graphs}

Esta tese/dissertação contém as correções e alterações sugeridas pela Comissão Julgadora durante a defesa realizada por Juan Gutiérrez em 20/12/2018.

O original encontra-se disponível no Instituto de Matemática e Estatística da Universidade de São Paulo.

Comissão Julgadora:

- Profa. Dra. Cristina Gomes Fernandes (orientadora) - IME-USP

- Prof. Dr. Fábio Happ Botler - UFRJ

- Profa. Dra. Christiane Neme Campos - UNICAMP

- Prof. Dr. Daniel Morgato Martin - UFABC

- Profa. Dra. Yoshiko Wakabayashi - IME-USP 


\section{Abstract}

The intention of this work is to study problems about transversals of graphs. A transversal of a graph is a set of vertices or edges that intersects every object of some type. We study three types of transversals: of longest paths, of longest cycles, and of triangles. For each such type of transversal, we show upper bounds on the minimum cardinality of a transversal in a given graph class.

The problems we study here have a strong connection with two well-known questions in graph theory: Gallai's question and Tuza's Conjecture. Gallai asked whether all longest paths in a connected graph intersect. In terms of transversals, Gallai was asking whether there is a transversal of longest paths of cardinality one. Although the answer to this question is negative, it is still open for several classes of graphs. One part of this work is as an attempt to solve Gallai's question, and its corresponding analogous question for cycles, on important classes of graphs. In some of these classes we are able to solve the question and in others we present significant advances.

Tuza conjectured whether the minimum cardinality of a transversal of triangles is at most twice the cardinality of a maximum packing of triangles, where a packing of triangles is a set of edge-disjoint triangles in a graph. This conjecture is still open and several related advances have been made in the literature. One part of this work is an attempt to solve Tuza's Conjecture for several classes of graphs. For some of these classes we prove the conjecture. For some other classes, the conjecture was already proved, so we show stronger results.

Keywords: graph theory, transversal, packing, longest paths, longest cycles, triangles. 


\section{Contents}

$\begin{array}{lll}1 & \text { Introduction } & 1\end{array}$

1.1 Longest Paths and Longest Cycles . . . . . . . . . . . . . . . . . . 2

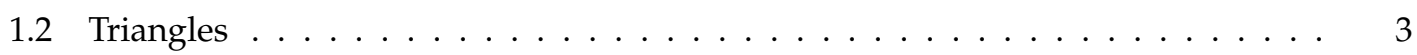

1.3 Outline of the Thesis $\ldots \ldots \ldots \ldots \ldots \ldots \ldots$

\begin{tabular}{|lll}
2 & Definitions, Notation, and Proof Techniques & 7
\end{tabular}

2.1 Basic Graph Theory . . . . . . . . . . . . . . . . . . . . 7

2.2 Tree Decomposition, $k$-trees, and Chordal Graphs . . . . . . . . . . . . . . . 11

2.3 Paths, Cycles, and Attractors . . . . . . . . . . . . . . . . . . . . 13

2.4 Branches . . . . . . . . . . . . . . . . . . . . . . . . . . . . . 15

2.5 Proof Techniques for Longest Paths and Longest Cycles . . . . . . . . . . . . 16

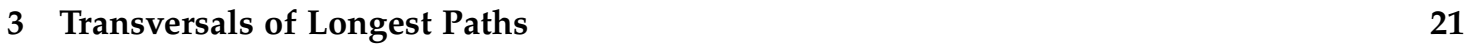

3.1 Chordal Graphs . . . . . . . . . . . . . . . . . . . . . . . . . 21

3.2 Partial $k$-Trees . . . . . . . . . . . . . . . . . . . . . . . 27

3.3 Full Substar Graphs $\ldots \ldots \ldots \ldots$. . . . . . . . . . . . . . . . 30

3.4 Bipartite Permutation Graphs $\ldots \ldots \ldots \ldots$. . . . . . . . . . . . 32

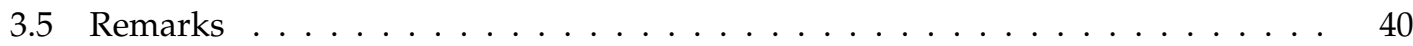

$\begin{array}{|ll|}4 & \text { Transversals of Longest Cycles }\end{array} 41$

$4.1 \quad$ Split Graphs . . . . . . . . . . . . . . . . . . . . . . . . . . . . . . 41

4.2 Chordal Graphs . . . . . . . . . . . . . . . . . . . . . . . . . 43

4.3 Partial $k$-Trees . . . . . . . . . . . . . . . . . . . . . . . . . . . 50

4.4 Partial 3-Trees . . . . . . . . . . . . . . . . . . . . . . . . . . . . . 53

4.4 .1 Proof of the main theorem . . . . . . . . . . . . . . 53

4.4 .2 New definitions . . . . . . . . . . . . . . . . . . 56

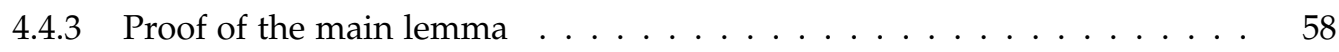

4.4 .4 Proof of the auxiliary lemmas $\ldots \ldots \ldots \ldots \ldots \ldots$

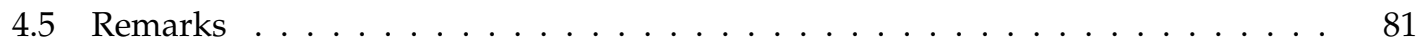

$\begin{array}{|ll|}5 & \text { Transversals of Triangles }\end{array}$ 


\section{vi CONTENTS}

5.1 Rooted Tree Decomposition . . . . . . . . . . . . . . . . . . . . . . 83

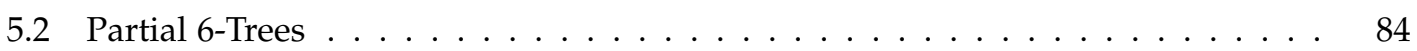

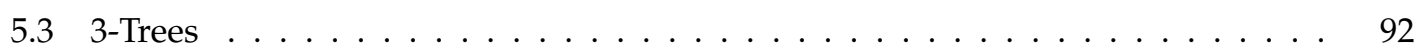

$5.4 \quad$ Planar Triangulations . . . . . . . . . . . . . . . . . . . . . . . 101

5.5 Dense Tripartite Graphs $\ldots \ldots \ldots$. . . . . . . . . . . . . . . . 103

5.6 Complete 4-Partite Graphs. . . . . . . . . . . . . . . . . . . . . . . . . . . 105

5.7 Remarks . . . . . . . . . . . . . . . . . . . 107

$\begin{array}{lll}6 & \text { Conclusions and Future Work } & 109\end{array}$

6.1 Longest Paths and Longest Cycles . . . . . . . . . . . . . . . . . . . . . . . 109

6.2 Triangles . . . . . . . . . . . . . . . . . . . . . . . . . 111

6.3 Other Types of Transversals $\ldots \ldots \ldots \ldots$

\begin{tabular}{ll}
\hline Bibliography & 115
\end{tabular}

$\begin{array}{ll}\text { Index } & 121\end{array}$ 


\section{Chapter 1}

\section{Introduction}

In Graph Theory, a classic problem consists in finding a set of vertices or edges that intersects every object of some type. Such a set is called a transversal, and we look for small transversals, possibly of minimum cardinality. Since determining the cardinality of a minimum transversal can be very hard, other approaches have been considered such as search for good upper bounds for its cardinality. In this thesis, our goal is to study some types of transversals in graphs. For example, transversals of longest paths, of longest cycles, and of triangles. Since it is difficult to obtain good bounds in general, we concentrate on specific graph classes.

By duality of linear programming, minimizing the cardinality of a transversal of some objects is related to maximizing the cardinality of a packing, that is, a set of pairwise disjoint such objects. In fact, it is always the case that the cardinality of a maximum packing is at most the cardinality of a minimum transversal. So, it is interesting to ask for the ratio between them.

If the object is a longest path (cycle), the maximum cardinality of a packing of vertex-disjoint longest paths (cycles) equals one, as a classic result states that every pair of longest paths (cycles) intersect each other in a connected graph (in a 2-connected graph). Hence, it is interesting to ask whether the cardinality of a transversal of longest paths (cycles) in a connected graph (in a 2-connected graph) equals one. Gallai raised this question for longest paths, that resulted in a negative answer from a small example. For longest cycles, the answer to the corresponding question is also negative. It remains open whether these questions have positive answers for some classes of graphs, or how large the cardinality of a minimum transversal can be.

If the object is a triangle, then the cardinality of a packing of edge-disjoint triangles can be arbitrarily large. Observe also that if we chose all the edges of all triangles of an arbitrary maximal packing, then we obtain a (not necessarily minimum) transversal. Hence, the cardinality of a minimum transversal is at most three times the cardinality of a maximum packing of triangles. A conjecture of Tuza states that there is a transversal of triangles of cardinality at most twice the cardinality of a maximum packing of triangles. 


\subsection{Longest Paths and Longest Cycles}

In Chapter 3 and Chapter 4 , we study vertex-transversals of longest paths and of longest cycles in graphs. That is, we look for small sets of vertices that intersect all longest paths, and small sets of vertices that intersect all longest cycles. Gallai [EK68] asked in 1966 if every connected graph has a longest path transversal of cardinality one. The answer to Gallai's question is negative. Figure 1.1 shows the example that was independently found by Walther and Voss [WV74] and Zamfirescu [Zam76].

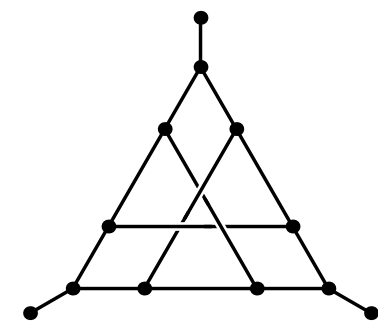

Figure 1.1: The classical 12-vertex example that yields a negative answer to Gallai's question. Observe that, for any vertex of the graph, there is a longest path that does not contain it. Thus, it is not the case that all longest paths intersect.

However, when we restrict attention to some specific classes of graphs, the answer to Gallai's question turns out to be positive. For example, it is well-known that any set of subtrees of a tree satisfies the Helly Property [Hel23, Hor72]. If we consider the set of subtrees consisting on the longest paths of the tree, since they are pairwise intersecting, we conclude that there is a vertex that belongs to all of them.

There are other graph classes which are known to yield a positive answer to Gallai's question. Klavžar and Petkovšek [KP90] proved that this is the case for connected split graphs, connected cacti, and connected graphs whose blocks are Hamilton-connected, almost Hamilton-connected or cycles. Balister et al. [BGLS04] proved the same for the class of connected interval graphs and also for its superclass of connected circular arc graphs, but there was a gap in their proof for the latter, that was then filled in by Joos [Joo15]. De Rezende et al. [dFMW13] proved that the answer to Gallai's question is positive for 2-trees and Chen et al. [CEF $\left.{ }^{+} 17\right]$ extended this result for connected seriesparallel graphs, also known as connected partial 2-trees. Chen [Che15] proved the same for connected graphs with matching number smaller than three, while Cerioli and Lima [CL16, Lim16] proved it for connected $P_{4}$-sparse graphs, connected $\left(P_{5}, K_{1,3}\right)$-free graphs, connected graphs that are the join of any two other graphs, and connected starlike graphs, a superclass of split graphs. Also, Jobson et al. [JKLW16] proved it for connected dually chordal graphs and connected cographs, and Golan and Shan [GS16] for connected $2 K_{2}$-free graphs.

A similar question can be asked, for 2-connected graphs, with cycles instead of paths. For this case, the answer is also negative in general, as the Petersen graph 
shows. Not so much attention has been given to this question as in the case of paths. However, Jobson et al. [JKLW16, Corollary 8] mentioned that their proof for paths in connected dually chordal graphs can be easily adapted to show that all longest cycles intersect in 2-connected dually chordal graphs, which includes doubly chordal, strongly chordal, and interval graphs. They also mention that their proof can be applied to show that all longest cycles intersect in 2-connected split graphs. Also, Fernandes and the author [FG17] showed that all longest cycles intersect in 3-trees. Other questions about intersections of longest cycles have also been raised by several authors [CFG98, Hip08, JS97, ST95].

As a common vertex to all longest paths (cycles) may not always exist, it is interesting to look for a set of vertices such that every longest path (cycle) has at least one vertex in that set. Such a set is called a longest path transversal (longest cycle transversal). The minimum cardinality of a longest path (cycle) transversal is denoted by $\operatorname{lpt}(G)(\operatorname{lct}(G))$. Since determining $\operatorname{lpt}(G)$ and $\operatorname{lct}(G)$ for a graph $G$ can be very hard, other approaches have been considered such as search for good upper bounds for their cardinalities.

In this direction, Thomassen [Tho78] showed that $\operatorname{lct}(G) \leq\lceil n / 3\rceil$ for any graph $G$ on $n$ vertices. Rautenbach and Sereni [RS14] proved that $\operatorname{lpt}(G) \leq\left\lceil\frac{n}{4}-\frac{n^{2 / 3}}{90}\right\rceil$ if $G$ is connected, that $\operatorname{lct}(G) \leq\left\lceil\frac{n}{3}-\frac{n^{2 / 3}}{36}\right\rceil$ if $G$ is 2-connected, that $\operatorname{lpt}(G) \leq 9 \sqrt{n} \lg n$ for planar graphs with at least 2 vertices, and that $\operatorname{lpt}(G) \leq k+1$ if $G$ has treewidth at most $k$. Van Aardt et al. [vABD $\left.{ }^{+} 15\right]$ showed that $\operatorname{lct}(G) \leq n / L$ for graphs whose longest cycles have length $L \leq 8$. Fernandes and the author [FG17] showed that $\operatorname{lct}(G) \leq 2$ if $G$ is a 2-connected partial 3-tree. It was proven by Tutte [Tut56] that every 4-connected planar graph is Hamiltonian, so $\operatorname{lpt}(G)=\operatorname{lct}(G)=1$ when $G$ is planar and 4-connected.

In this thesis, we give results for $\operatorname{lpt}(G)$ and $\operatorname{lct}(G)$ when $G$ is chordal (Section 3.1 and Section 4.2), and when $G$ is a partial $k$-tree (Section 3.2 and Section 4.3). We also give results for $\operatorname{lpt}(G)$ when $G$ is a full substar graph (Section 3.3) or a bipartite permutation graph (Section 3.4), and for $\operatorname{lct}(G)$ when $G$ is a split graph (Section 4.1) or a partial 3-tree (Section 4.4).

\subsection{Triangles}

In Chapter 5, we study edge-transversals of triangles in graphs. That is, we look for small sets of edges that intersect all triangles and their relation with their natural dual. Formally, a triangle transversal of a graph $G$ is a set of edges of $G$ such that all triangles in $G$ have at least one edge in that set. A triangle packing of $G$ is a set of edge-disjoint triangles in $G$. For every graph $G$, define $\tau(G)$ as the cardinality of a minimum triangle transversal in $G$, and $v(G)$ as the cardinality of a maximum triangle packing in $G$. Note that $\tau(G) \geq v(G)$. Indeed, as any triangle transversal of $G$ must have at least one edge in every triangle of a maximum triangle packing in $G$. Note also that $\tau(G) \leq 3 v(G)$. Indeed, the union of the edges of the triangles in any maximum triangle packing is a 
triangle transversal in $G$.

In 1981, Tuza [Tuz81] proposed the following conjecture.

Conjecture 1.1 (Tuza's Conjecture). For every graph $G, \tau(G) \leq 2 v(G)$.

Tuza's Conjecture has been studied broadly. In 1990, Tuza himself [Tuz90] proved his conjecture for planar graphs, graphs with $n$ vertices and at least $\frac{7}{16} n^{2}$ edges (which include complete graphs with at least 8 vertices), and line graphs of triangle-free graphs. For chordal graphs, he proved that his conjecture is true when the graph is $K_{5}$-free. Also, if $G$ is a $K_{4}$-free chordal graph, he proved that $\tau(G)=v(G)$. Tuza's Conjecture is tight in the sense that there exist infinitely many graphs for which $\tau(G)=2 v(G)$. Indeed, $\tau\left(K_{4}\right)=2 v\left(K_{4}\right)=2$, and $\tau\left(K_{5}\right)=2 v\left(K_{5}\right)=4$. So, when the blocks of $G$ are isomorphic to $K_{4}, K_{5}$, or $K_{2}$, the conjecture is tight. However, until now, there is no characterization of the class of graphs for which the equality holds. Haxell [Hax99] showed the first and unique nontrivial bound to Tuza's Conjecture. She showed that $\tau(G) \leq 2.87 \nu(G)$ for every graph $G$.

Tuza [Tuz90] also showed that $\tau(G) \leq 2.34 v(G)$ for a tripartite graph $G$. This result was improved by Haxell and Kohayakawa [HK98], who proved not only Tuza's Conjecture for tripartite graphs, but proved that $\tau(G) \leq 1.956 v(G)$ for every tripartite graph $G$. They remarked that finding a tripartite graph $G$ with $\tau(G) / \nu(G)$ much larger than one seemed difficult. They found tripartite graphs that satisfy $\tau(G)=\frac{5}{4} v(G)$. The best known result on tripartite graphs is by Szestopalow [Sze16]. He showed that $\tau(G) \leq 1.87 v(G)$ if $G$ is tripartite.

In trying to generalize the result for tripartite graphs, Aparna et al. [ALBT11] showed that for odd-wheel-free graphs, Tuza's Conjecture holds. Observe that this is indeed a stronger result because a 4-clique is a wheel with three external vertices. In the same paper, they defined the class of triangle-3-colorable graphs. These are graphs whose edges can be colored with three colors in such a way that each triangle receives three distinct colors on its edges. They proved that Tuza's Conjecture holds for this class and, as a corollary, they showed that 4-colourable graphs satisfy Tuza's Conjecture. Also, they showed that, for every $\left(K_{4}\right.$, gem $)$-free graph $G$ (a gem graph is a graph formed by the union of a star with 5 vertices and a path that joins all vertices of the star that are not the center), the equality $\tau(G)=v(G)$ holds.

In another paper of the same authors, Aparna et al. [ALBT16] showed Tuza's Conjecture for graphs whose triangle graph is perfect. However, they mentioned that Gregory Puleo told them that this result can be derived from another result proved by him. Puleo [Pul15] considered an important measure of sparseness, the maximum average degree of a graph $G$, denoted by $\operatorname{Mad}(G)$, and defined as $\operatorname{Mad}(G)=\max \left\{\frac{2|E(H)|}{V(H)}: H \subseteq G\right\}$. He showed that, if $\operatorname{Mad}(G) \leq 7$, then $G$ satisfies Tuza's Conjecture, by using a known technique called "the discharging method" [CW16]. In the same paper, as an intermedi- 
ate result, Puleo showed that every minimal counterexample to Tuza's Conjecture has minimum degree at least 5 .

As we mentioned, Tuza showed his conjecture for planar graphs [Tuz90]. Cui et al. [CHM09] characterized planar graphs for which Tuza's Conjecture is tight. Haxell et al. [HKT12a] showed that, when $G$ is a $K_{4}$-free planar graph, the stronger inequality $\tau(G) \leq \frac{3}{2} v(G)$ holds. Moreover, equality is attained only for a particular class of graphs. They also proved that the same statement is true if, instead of $G$ being planar, each edge of $G$ belongs to at most two triangles.

A generalization of the result on planar graphs was shown by Krivelevich [Kri95]. He proved that Tuza's Conjecture holds for $K_{3,3}$-free graphs. Another generalization for planar graphs happened as follows. Puleo [Pul15] mentioned that, by a theorem of Wagner [Wag37], the Four-Color Theorem implies that all graphs with no $K_{5}$-minor are 4-colorable. So the proof for 4-colorable graphs by Aparna et al. [ALBT11] implies that Tuza's Conjecture is also valid for graphs with no $K_{5}$-minor. Moreover, Puleo [Pul15] extended this result and showed that Tuza's Conjecture holds for graphs with no $K_{5}$-subdivision.

Krivelevich [Kri95] studied a fractional version of Tuza's Conjecture. Let $v^{*}(G)$ and $\tau^{*}(G)$ be the corresponding fractional versions of $v(G)$ and $\tau(G)$. The fact that $\tau^{*}(G) \leq 2 v^{*}(G)$ is obvious from linear programming duality. He proved that $\tau(G) \leq 2 \tau^{*}(G)$ and that $v^{*}(G) \leq 2 v(G)$. The bound for the latter is tight, since $v^{*}\left(K_{4}\right)=2$ and $v\left(K_{4}\right)=1$. For the former, Chapuy et al. [CDM$\left.{ }^{+} 14\right]$ showed that $\tau^{*}(G) \leq 2 \tau(G)-\frac{1}{\sqrt{6}} \sqrt{\tau^{*}(G)}$, and that this bound is essentially best possible. Haxell $e t$ al. [HKT12b] proved that $\tau^{*}(G) \leq 1.8 \tau(G)$ for every $K_{4}$-free graph $G$.

Chapuy et al. $\left[\mathrm{CDM}^{+} 14\right]$ generalized this conjecture to its natural capacited analogue. That is, they considered integers capacities on the edges, so now the triangles in the collection do not have to be disjoint, but the capacity of each edge bounds the number of triangles with this edge that can exist in the collection. They showed that this new conjecture is equivalent to Tuza's Conjecture in multigraphs instead of simple graphs. In their paper, they showed that the capacited version of the conjecture is true for planar graphs. Also, they generalized the proof given by Haxell and proved a bound slightly less than 3 for general graphs.

In this thesis, we give novel results regarding Tuza's Conjecture (Chapter 5). We prove Tuza's Conjecture for graphs with treewidth at most 6 (Section 5.2). We also show stronger results for other classes of graphs, namely, 3-trees (Section 5.3), planar triangulations (Section 5.4), dense tripartite graphs (Section 5.5), and complete 4-partite graphs (Section 5.6). 


\subsection{Outline of the Thesis}

We begin with a preliminary chapter (Chapter 2). In this chapter we review basic definitions in graph theory and present basic preliminary results on longest paths and longest cycles that are used in the next two chapters (Chapters 3 and 4 ). The following chapters present the results obtained for each of the transversals considered.

In Chapter 3 we present our results on transversals of longest paths. We show results for chordal graphs (Section 3.1), partial $k$-trees (Section 3.2), full substar graphs (Section 3.3) and bipartite permutation graphs (Section 3.4). All these results appear in a joint work with Márcia R. Cerioli, Cristina G. Fernandes, Renzo Gómez and Paloma Lima. The results of this chapter were presented at the IX Latin and American Algorithms, Graphs and Optimization Symposium (LAGOS 2017) under the title "Transversals of Longest Paths" $\mathrm{CFG}^{+} 17$.

In Chapter 4 we present our results on transversals of longest cycles. We give results for split graphs (Section 4.1), chordal graphs (Section 4.2), partial $k$-trees (Section 4.3) and partial 3-trees (Section 4.4). The results of this chapter were presented at the XIII Latin American Theoretical INformatics Symposium (LATIN 2018) under the title "Transversals of longest cycles in chordal and bounded tree-width graphs" [Gut18].

In Chapter 5 we present our results on transversals of triangles. A special tool called "rooted tree decomposition" is presented at the beginning of this chapter (Section 5.1). We then proceed to show our results for partial 6-trees (Section 5.2), 3-trees (Section 5.3), planar triangulations (Section 5.4), dense tripartite graphs (Section 5.5) and complete 4-partite graphs (Section 5.6). All these results are a joint work with Fábio Botler and Cristina G. Fernandes. The result of Section 5.2 was presented at the III Encontro de Teoria da Computação (ETC 2018) do XXXVIII Congresso da Sociedade Brasileira de Computação (CSBC 2018) under the title "On Tuza's conjecture for graphs with treewidth at most 6" [BFG18]. 


\section{Chapter 2}

\section{Definitions, Notation, and Proof Techniques}

This chapter introduces basic concepts that serve as a basis for the next chapters. In Section 2.1 we give basic graph theory definitions. The reader may choose to skip this section and consult it only when necessary. The next sections can be viewed as preliminaries to Chapters 3, 4, and 5. In Section 2.3 we give specific definitions on paths and cycles, that are used in Chapters 3 and 4 . In Section 2.5 we give core lemmas to be used in Chapters 3 and 4

\subsection{Basic Graph Theory}

A graph $G$ is an ordered pair $(V(G), E(G))$ consisting of a set $V(G)$ of vertices and a set $E(G)$ of edges, where an edge is an unordered pair of distinct vertices. For notational simplicity, an edge $\{u, v\}$ is denoted by $u v$ or $v u$. We also say that $u$ and $v$ are adjacent, and that $u$ and $v$ are the ends of $u v{ }^{1}$ (Figure 2.1.(a)).

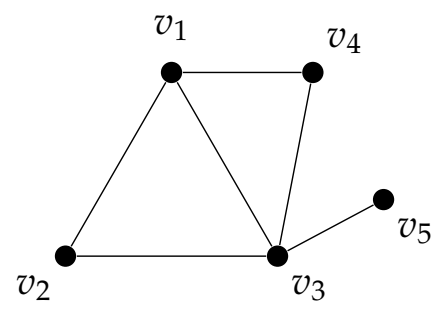

(a)

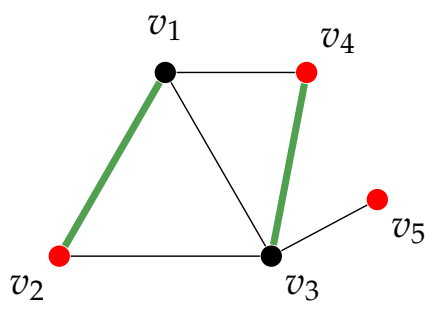

(b)

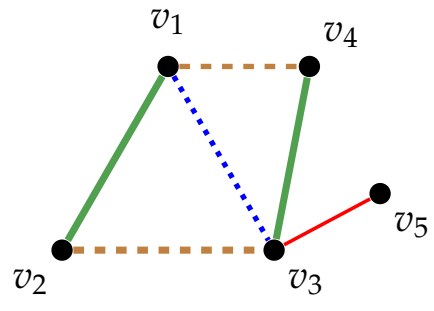

(c)

Figure 2.1: (a) A graph $G$ with $V(G)=\left\{v_{1}, v_{2}, v_{3}, v_{4}, v_{5}\right\}$ and $E(G)=$ $\left\{v_{1} v_{2}, v_{1} v_{3}, v_{1} v_{4}, v_{2} v_{3}, v_{3} v_{4}, v_{3} v_{5}\right\}$. (b) $\left\{v_{2}, v_{4}, v_{5}\right\}$ is an independent set in $G$ and $\left\{v_{1} v_{2}, v_{3} v_{4}\right\}$ is a matching in $G$. (c) $\left\{\left\{v_{1} v_{2}, v_{3} v_{4}\right\},\left\{v_{1} v_{4}, v_{2} v_{3}\right\},\left\{v_{1} v_{3}\right\},\left\{v_{3} v_{5}\right\}\right\}$ is an edge-coloring in $G$. We can think that an edge-coloring is an assignment of colours to the edges of a graph such that no two edges with the same colour share a vertex. In this example, we use four colours for $G$.

The neighbors of a vertex $v$ are the vertices adjacent to $v$ in $G$ and this set is denoted by $N_{G}(v)$. The degree of $v$ is the number of vertices of $N_{G}(v)$ and is denoted by $d_{G}(v)$.

\footnotetext{
${ }^{1}$ In other sources, this object is named as simple graph. The reader may find references when the term graph refers to multigraphs, which are graphs that allow distinct edges with the same ends.
} 
If the context is clear, we write $N(v)$ and $d(v)$. An independent set in a graph $G$ is a set of vertices that are pairwise not adjacent. Two edges in $G$ are adjacent if they have an end in common. A matching in a graph $G$ is a set of edges that are pairwise not adjacent (Figure 2.1(b)). An edge-coloring of a graph $G$ is a collection $\left\{M_{1}, M_{2}, \ldots, M_{k}\right\}$ of pairwise disjoint matchings in $G$ such that $M_{1} \cup M_{2} \cup \cdots \cup M_{k}=E(G)$ (Figure 2.1(c)). Given two graphs $G$ and $H$, we say that $G \simeq H$ if there is a bijection $f: V(G) \rightarrow V(H)$ such that $u v \in E(G)$ if and only if $f(u) f(v) \in E(H)$.

A graph $G$ is called complete if all vertices of $G$ are pairwise adjacent. A complete graph on $n$ vertices is denoted by $K_{n}$. A graph $P$ is called a path if $V(P)$ admits a permutation $\left(v_{1}, v_{2}, \ldots, v_{k}\right)$ such that $E(P)=\left\{v_{1} v_{2}, v_{2} v_{3}, \ldots, v_{k-1} v_{k}\right\}$. We denote such a path by $v_{1} v_{2} \cdots v_{k}$. Vertices $v_{1}$ and $v_{k}$ are the extremes of $P$, and all other vertices are the internal vertices of $P$. We also say that $P$ joins $v_{1}$ and $v_{k}$. A graph $C$ is called a cycle if it has at least three vertices and $V(C)$ admits a permutation $\left(v_{1}, v_{2}, \ldots, v_{k}\right)$ such that $E(C)=\left\{v_{1} v_{2}, v_{2} v_{3}, \ldots, v_{k-1} v_{k}, v_{k} v_{1}\right\}$. We can denote such cycle by $v_{1} v_{2} \cdots v_{k} v_{1}$. The length of a path or a cycle $H$ is the number $|E(H)|$, and it is denoted by $|H|$ (Figure 2.2). Two paths $P_{1}$ and $P_{2}$ are internally disjoint if they do not share any internal vertex. A path $P$ is internally disjoint from a cycle $C$ if $C$ does not contain any internal vertex of $P$.

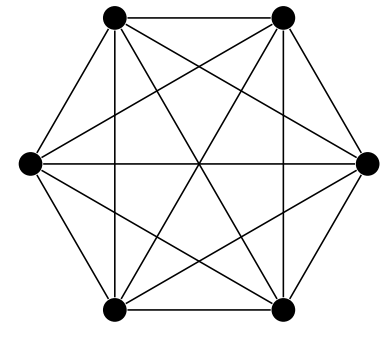

(a)

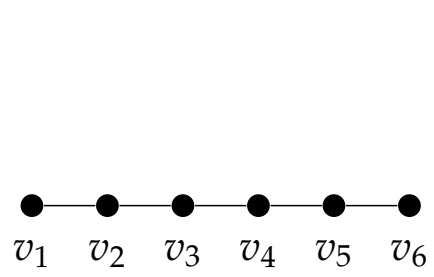

(b)

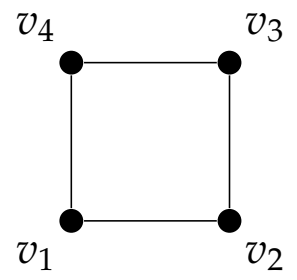

(c)

Figure 2.2: (a) A complete graph on six vertices, denoted by $K_{6}$. (b) A path $v_{1} v_{2} v_{3} v_{4} v_{5} v_{6}$ of length five. (c) A cycle $v_{1} v_{2} v_{3} v_{4} v_{1}$ of length four.

A graph $H$ is a subgraph of a graph $G$ if $V(H) \subseteq V(G)$ and $E(H) \subseteq E(G)$. If $H$ is a subgraph of $G$, we write $H \subseteq G$. Let $U \subseteq V(G)$. The subgraph of $G$ induced by $U$ is the graph $(U, F)$, where $F$ is the set of edges of $G$ with both ends in $U$. Such subgraph is denoted by $G[U]$. The graph $G[V(G) \backslash U]$ is denoted by $G-U$ (Figure 2.3(b)). If $v \in V(G)$, we abbreviate $G-\{v\}$ by $G-v$ (Figure 2.3(c)). Let $X \subseteq E(G)$. The graph $(V(G), E(G) \backslash X)$ is denoted by $G-X$. If $e \in E(G)$, we abbreviate $G-\{e\}$ by $G-e$ (Figure 2.3(d)). The complement of a graph $G$ on $n$ vertices is the graph $K_{n}-E(G)$, and it is denoted by $\bar{G}$ (Figure 2.3(e)). 


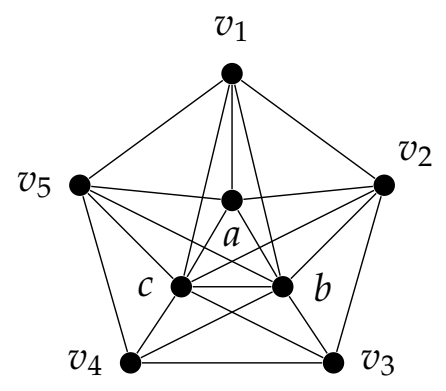

(a)

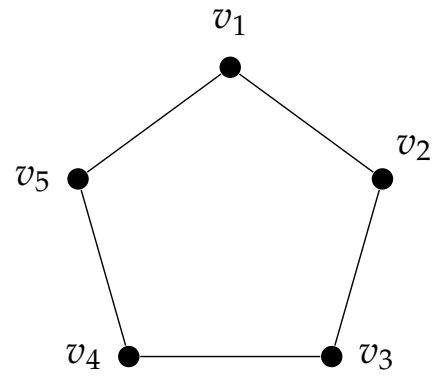

(b)

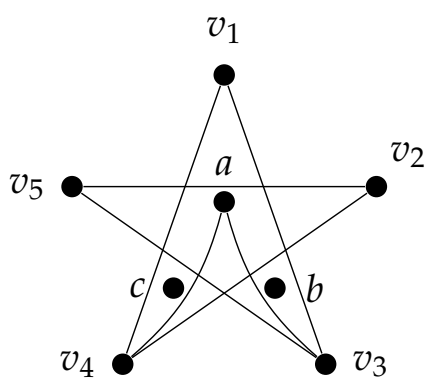

(e)

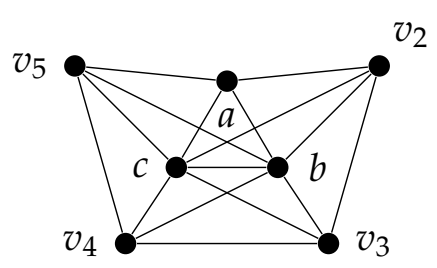

(c)

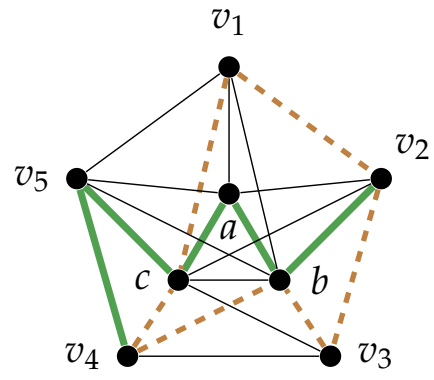

(f)

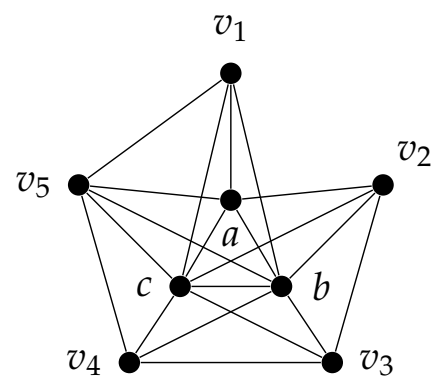

(d)

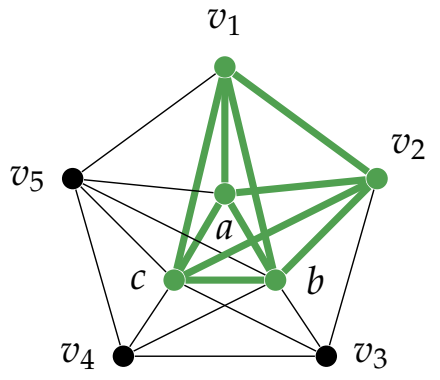

(g)

Figure 2.3: (a) A graph $G$. (b) The graph $G-\{a, b, c\}$. (c) The graph $G-v_{1}$. (d) The graph $G-$ $v_{1} v_{2}$. (e) The graph $\bar{G}$. (f) $v_{4} v_{5} c a b v_{2}$ is a path in $G$ and $v_{1} v_{2} v_{3} b v_{4} c v_{1}$ is a cycle in $G$. (g) $a b c$ is a triangle in $G$ and $\left\{v_{1}, v_{2}, a, b, c\right\}$ is a 5 -clique in $G$. Moreover, $\omega(G)=5$.

Given a graph $G$ and a subgraph $H$ of $G$, we say that $H$ is a path (cycle) in $G$ if $H$ is a path (cycle) (Figure 2.3(f)). If $H$ is a complete graph, we say that $V(H)$ is a clique in $G$. We also abuse notation and say that $H$ is a clique. If $H$ is a complete graph with $k$ vertices, we say that $H$ is a $k$-clique in $G$. It is denoted by $\omega(G)$ the number of vertices of a maximum clique in $G$. If $H$ is a complete graph with three vertices, we say that $H$ is a triangle in $G$. When the context is clear, if $\Delta$ is a triangle in $G$ with $V(\Delta)=\{a, b, c\}$, then we write $\Delta=a b c$ (Figure $2.3(\mathrm{~g})$ ).

A graph $G$ is connected if, for every two vertices $u, v \in V(G)$, there exists a path in $G$ that joins $u$ and $v$. A graph $G$ is 2-connected if, for every two vertices $u, v \in V(G)$, there exists two internally disjoint paths in $G$ that joins $u$ and $v$. A component of a graph $G$ is 
any subgraph of $G$ that is connected and is maximal. An edge $e \in E(G)$ is called a bridge if the number of components of $G-e$ is one more than the number of components of $G$. Let $G$ be a graph and let $S \subset V(G)$. We say that $S$ separates vertices $u$ and $v$ if $u$ and $v$ are in different components of $G-S$. Let $X \subset V(G)$. We say that $S$ separates $X$ if $S$ separates at least two vertices of $X$ (Figure 2.4).

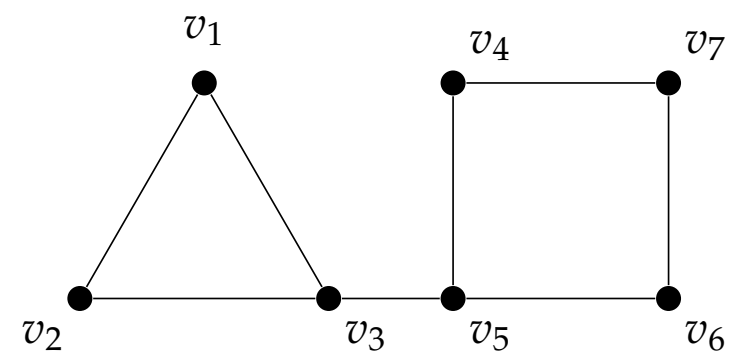

Figure 2.4: A connected graph $G$ with 7 vertices. Let $S=\left\{v_{3}, v_{5}\right\}$. Then $S$ separates $v_{1}$ and $v_{7}$. Let $X_{1}=\left\{v_{1}, v_{3}, v_{7}\right\}$ and $X_{2}=\left\{v_{1}, v_{3}, v_{5}\right\}$. Then $S$ separates $X_{1}$ but not $X_{2}$. Note also that $v_{3} v_{5}$ is a bridge in $G$.

A graph $T$ is called a tree if $T$ is connected and there exists no cycles in $T$. A leaf of $T$ is a vertex in $T$ of degree one. A digraph $G$ is an ordered pair $(V(G), E(G))$ consisting of a set $V(G)$ of nodes and a set $E(G)$ of arcs, where an arc is an ordered pair of distinct nodes. For notational simplicity, an $\operatorname{arc}(u, v)$ is denoted by $u v$. Given a tree $T$, a partial orientation of $T$ is a digraph $T^{\prime}$ such that $V\left(T^{\prime}\right)=V(T)$ and, if $u v \in E\left(T^{\prime}\right)$, then $u v \in E(T)$ (Figure 2.5).

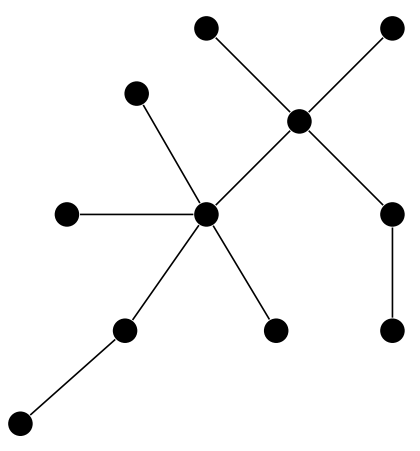

(a)

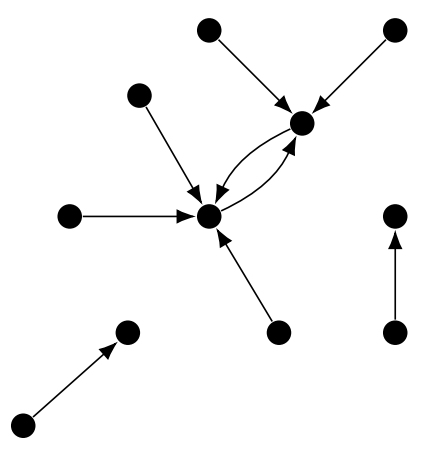

(b)

Figure 2.5: (a) A tree $T$. (b) A partial orientation $T^{\prime}$ of $T$. Note that $V(T)=V\left(T^{\prime}\right)$ but not all edges of $T$ are present in $T^{\prime}$ as arcs.

A graph $G$ is called $k$-partite if $V(G)$ can be partitioned into $k$ independent sets. If $\left\{X_{1}, X_{2}, \ldots, X_{k}\right\}$ is such a partition, then $G$ is denoted by $\left(X_{1}, X_{2}, \ldots, X_{k}, E(G)\right)$. We say that $G$ is complete k-partite if, for every pair $X_{i}, X_{j}$ in the partition, $G\left[X_{i} \cup X_{j}\right]$ has $\left|X_{i}\right|\left|X_{j}\right|$ edges. We also call a 2-partite graph a bipartite graph and a 3-partite graph 
a tripartite graph. A complete bipartite graph on the bipartition $\{A, B\}$ is denoted by $K_{|A|,|B|}$. A star is a complete bipartite graph $K_{1, k}$, for some integer $k$. If $k \geq 2$, we call the unique vertex of degree $k$ the center of the star. If $k=1$, we pick an arbitrary vertex to be the center of the star. Given a subgraph $S$ of $G$, we say that $S$ is a star in $G$ if $S$ is a star (Figure 2.6.).

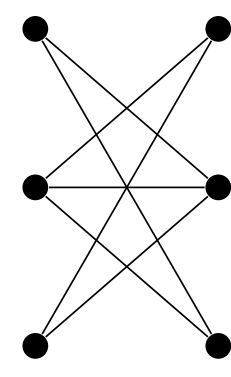

(a)

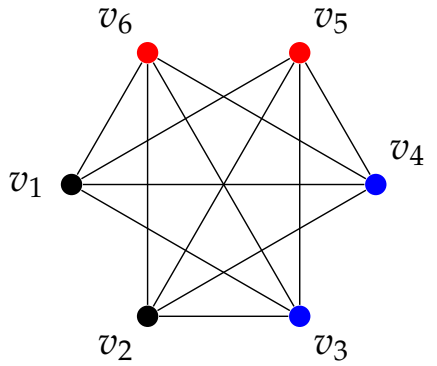

(b)

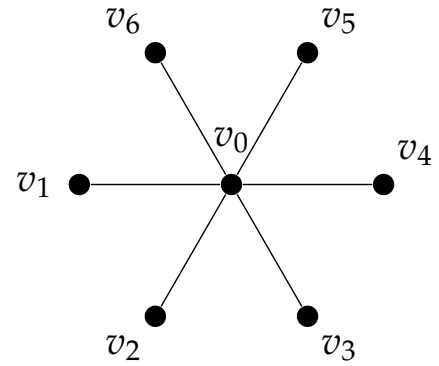

(c)

Figure 2.6: (a) A bipartite graph. (b) A complete tripartite graph with $\left\{\left\{v_{1}, v_{2}\right\},\left\{v_{3}, v_{4}\right\},\left\{v_{5}, v_{6}\right\}\right\}$ as the corresponding partition of its vertex set. (c) A star with center $v_{0}$. We can think that a $k$-partite graph is a graph that can be assigned $k$ colors to its vertices such that no two vertices with the same color are adjacent. In this sense, a color is assigned to each set in the partition of the graph.

\subsection{Tree Decomposition, $k$-trees, and Chordal Graphs}

A tree decomposition [Die10, p. 337] of a graph $G$ is a pair $(T, \mathcal{V})$, consisting of a tree $T$ and a collection $\mathcal{V}=\left\{V_{t}: t \in V(T)\right\}$ of (different) bags $V_{t} \subseteq V(G)$, that satisfies the following three conditions:

(T1) $\bigcup_{t \in V(T)} V_{t}=V(G)$;

(T2) for every $u v \in E(G)$, there exists a bag $V_{t}$ such that $u, v \in V_{t}$;

(T3) if a vertex $v$ is in two different bags $V_{t_{1}}, V_{t_{2}}$, then $v$ is also in any bag $V_{t}$ such that $t$ is on the (unique) path from $t_{1}$ to $t_{2}$ in $T$.

The width of $(T, \mathcal{V})$ is the number

$$
\max \left\{\left|V_{t}\right|-1: t \in V(T)\right\},
$$

and the treewidth $t w(G)$ of $G$ is the minimum width of any tree decomposition of $G$. To avoid confusion, we refer to the vertices of $T$ as nodes.

Sometimes we are interested in particular tree decompositions. If $G$ is a graph with treewidth $k$, then we say that $(T, \mathcal{V})$ is a full tree decomposition of $G$ if $\left|V_{t}\right|=k+1$ for every $t \in V(T)$, and $\left|V_{t} \cap V_{t^{\prime}}\right|=k$ for every $t t^{\prime} \in E(T)$ (Figure 2.7). 
Proposition 2.1 ([Bod98, Lemma 8][Gro14, Theorem 2.6]). Every graph has a full tree decomposition.

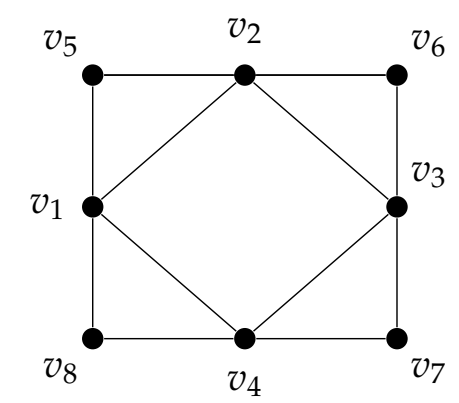

(a)

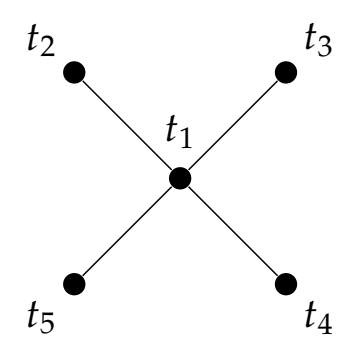

(b)

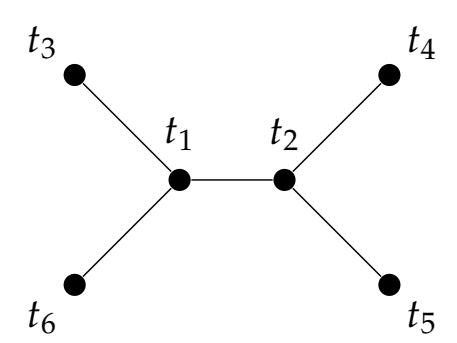

(c)

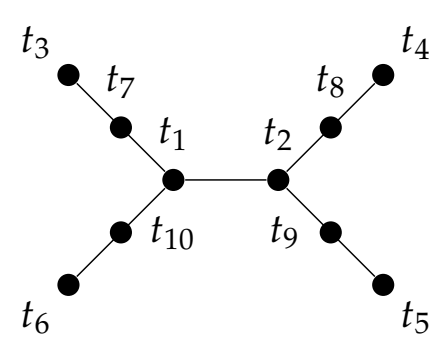

(d)

Figure 2.7: (a) A graph $G$ with treewidth two. We consider three tree decompositions of $G$ : $\left(T_{1}, \mathcal{V}_{1}\right)$ of width $3,\left(T_{2}, \mathcal{V}_{2}\right)$ of width 2 and $\left(T_{3}, \mathcal{V}_{3}\right)$ of width 2 . Only $\left(T_{2}, \mathcal{V}_{2}\right)$ is a full tree decomposition. Next we describe each of the tree decompositions mentioned. (b) $V\left(T_{1}\right)=$ $\left\{t_{1}, t_{2}, t_{3}, t_{4}, t_{5}\right\}, V_{t_{1}}=\left\{v_{1}, v_{2}, v_{3}, v_{4}\right\}, V_{t_{2}}=\left\{v_{1}, v_{2}, v_{5}\right\}, V_{t_{3}}=\left\{v_{2}, v_{3}, v_{6}\right\}, V_{t_{4}}=\left\{v_{3}, v_{4}, v_{7}\right\}$, and $V_{t_{5}}=\left\{v_{4}, v_{1}, v_{8}\right\}$. (c) $V\left(T_{2}\right)=\left\{t_{1}, t_{2}, t_{3}, t_{4}, t_{5}, t_{6}\right\}, V_{t_{1}}=\left\{v_{1}, v_{2}, v_{4}\right\}, V_{t_{2}}=\left\{v_{2}, v_{3}, v_{4}\right\}$, $V_{t_{3}}=\left\{v_{1}, v_{2}, v_{5}\right\}, V_{t_{4}}=\left\{v_{2}, v_{3}, v_{6}\right\}, V_{t_{5}}=\left\{v_{3}, v_{4}, v_{7}\right\}$, and $V_{t_{6}}=\left\{v_{1}, v_{4}, v_{8}\right\}$. (d) $V\left(T_{3}\right)=$ $\left\{t_{1}, t_{2}, t_{3}, t_{4}, t_{5}, t_{6}, t_{7}, t_{8}, t_{9}, t_{10}\right\}, V_{t_{1}}=\left\{v_{1}, v_{2}, v_{4}\right\}, V_{t_{2}}=\left\{v_{2}, v_{3}, v_{4}\right\}, V_{t_{3}}=\left\{v_{1}, v_{2}, v_{5}\right\}, V_{t_{4}}=$ $\left\{v_{2}, v_{3}, v_{6}\right\}, V_{t_{5}}=\left\{v_{3}, v_{4}, v_{7}\right\}, V_{t_{6}}=\left\{v_{1}, v_{4}, v_{8}\right\}, V_{t_{7}}=\left\{v_{1}, v_{2}\right\}, V_{t_{8}}=\left\{v_{2}, v_{3}\right\}, V_{t_{9}}=\left\{v_{3}, v_{4}\right\}$, and $V_{t_{10}}=\left\{v_{1}, v_{4}\right\}$.

A $k$-tree is defined recursively as follows. The complete graph on $k$ vertices is a $k$-tree. Any graph obtained from a $k$-tree by adding a new vertex and making it adjacent to exactly all the vertices of an existing $k$-clique is also a $k$-tree (Figure 2.8). A graph $G$ is a partial $k$-tree if and only if $G$ is the subgraph of a $k$-tree. Partial $k$-trees are closely related to the definition of tree decomposition, as the following proposition shows.

Proposition 2.2 ([Bod98, Theorem 35]). A graph $G$ is a partial $k$-tree if and only if $\operatorname{tw}(G) \leq k$. 


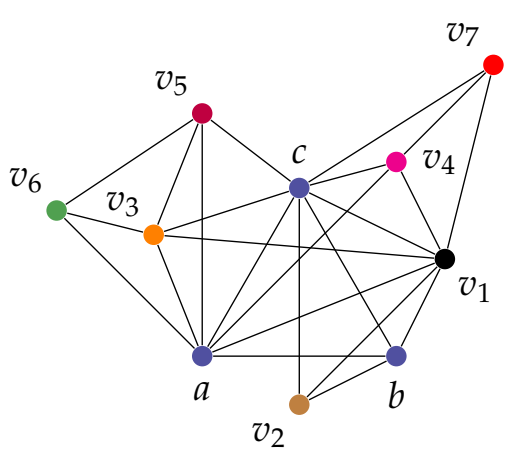

(a)

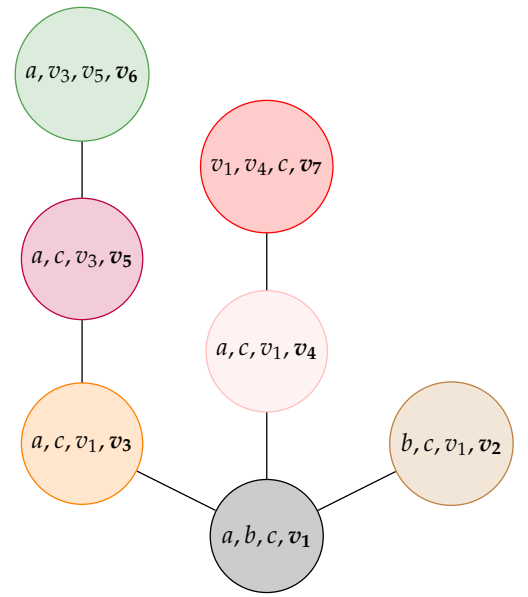

(b)

Figure 2.8: (a) A 3-tree $G$. To construct $G$, we begin with triangle $a b c$ and add the following sequence of vertices: $v_{1}-v_{2}-v_{3}-v_{4}-v_{5}-v_{6}-v_{7}$. (b) We can obtain a tree decomposition for $G$ in the following way: each time we add a new vertex, say $v_{i}$, to an already existing triangle, say $x y z$, we also add a new node, with corresponding bag $\left\{x, y, z, v_{i}\right\}$, to the tree decomposition and we make it adjacent to an already existing node whose corresponding bag contains $x, y$ and $z$. Moreover, the tree decomposition obtained by this proceeding is a full tree decomposition for $G$, as $G$ has treewidth 3 by Proposition 2.4

A graph is called chordal if every induced cycle has length three. The next proposition is a direct consequence of Corollary 12.3.12 in Diestel [Die10].

Proposition 2.3. For every chordal graph $G, \operatorname{tw}(G)=\omega(G)-1$.

Note that $k$-trees are chordal [Gol04, Theorem 4.1]. Thus, by Propositions 2.2 and 2.3 , we have the next proposition.

Proposition 2.4. Any $k$-tree with at least $k+1$ vertices has treewidth $k$.

Next we define a special tree decomposition for chordal graphs. A tree decomposition $(T, \mathcal{V})$ of a graph $G$ is called a clique tree if $\mathcal{V}$ is the set of all maximal cliques in $G$ (Figure 2.9).

Proposition 2.5 ([Gav74, Theorem 2, Theorem 3]). Every chordal graph G has a clique tree.

\subsection{Paths, Cycles, and Attractors}

Given two internally disjoint paths $P$ and $Q$ that share (at least) one of their ends, the path or cycle $(V(P) \cup V(Q), E(P) \cup E(Q))$ is denoted by $P$. Q. For a vertex $v$ in a path $P$, let $P^{\prime}$ and $P^{\prime \prime}$ be the paths such that $P=P^{\prime} \cdot P^{\prime \prime}$ with $V\left(P^{\prime}\right) \cap V\left(P^{\prime \prime}\right)=\{v\}$. We refer to these two paths as the $v$-tails of $P$. For a pair of vertices $\{a, b\}$ in a cycle $C$, let $C^{\prime}$ and $C^{\prime \prime}$ be the paths such that $C=C^{\prime} \cdot C^{\prime \prime}$ with $V\left(C^{\prime}\right) \cap V\left(C^{\prime \prime}\right)=\{a, b\}$. We refer to these two 


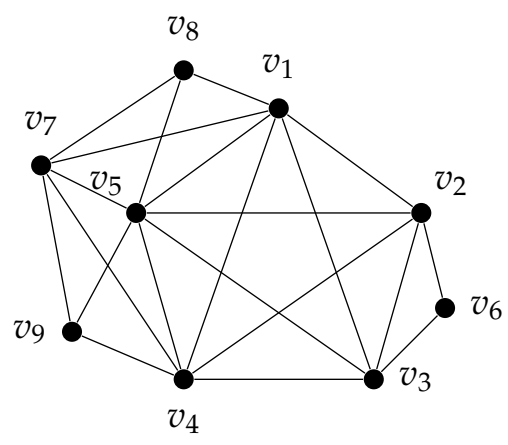

(a)

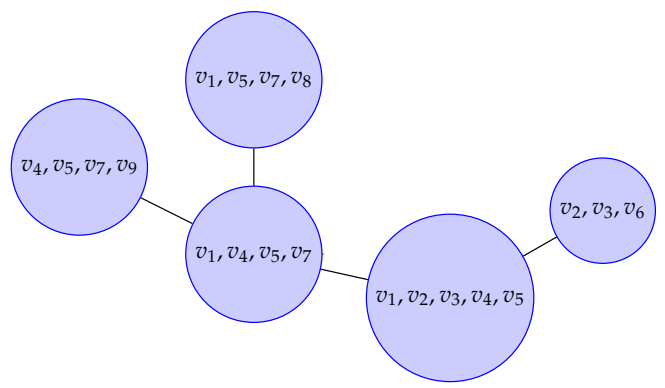

(b)

Figure 2.9: (a) A chordal graph $G$. As $\omega(G)=5, \operatorname{tw}(G)=4$ by Proposition 2.3. (b) A representation of a clique tree of $G$. Observe that the bags correspond to all maximal cliques in $G$.

paths as the $a b$-parts of $C$. Moreover, we can extend this notation and define, for a triple (4-tuple) of vertices $\{a, b, c\}(\{a, b, c, d\})$ in a cycle $C$, the abc-parts of $C$ (abcd-parts) of $C$. Given a path $P$ that contains vertices $a$ and $b$, when the context is clear, it is denoted by $P_{a}$ the $a$-tail of $P$ that does not contain $b$ and by $P_{b}$ the $b$-tail of $P$ that does not contain $a$. Also, if the context is clear, it is denoted by $\widetilde{P}$ the subpath of $P$ that has $a$ and $b$ as its extremes. In other words, $P=P_{a} \cdot \widetilde{P} \cdot P_{b}$. Given a cycle $C$ that contains vertices $a, b$ and $c$, when the context is clear, it is denoted by $C_{a b}$ the $a b$-part of $C$ that does not contain $c$, by $C_{b c}$ the $b c$-part of $C$ that does not contain $a$, and by $C_{a c}$ the $a c$-part of $C$ that does not contain $b$.

Let $S$ be a set of vertices in a graph $G$. We say that a path (cycle) $P$ k-intersects $S$ if $|V(P) \cap S|=k$. Moreover, we also say that $P k$-intersects $S$ at $V(P) \cap S$. A path (cycle) $P$ crosses a set $S$ of vertices if $S$ separates $V(P)$ in $G$. Otherwise, $S$ fences $P$. If $P$ crosses $S$ and $k$-intersects $S$, then we say that $P k$-crosses $S$. We also say that $P k$-crosses $S$ at $V(P) \cap S$. If $P$ is fenced by $S$ and $k$-intersects $S$, then we say that $P$ is $k$-fenced by $S$. If two paths (cycles) $P$ and $Q$ are such that $V(P) \cap S=V(Q) \cap S$, then we say they are S-equivalent (Figures 2.10(a) and 2.10(b)).

If $P$ and $Q$ are paths fenced by $S$ in a graph $G$, we write $P \sim_{S} Q$ if there exist vertices $u \in V(P)$ and $v \in V(Q)$ such that $u$ and $v$ are in the same component of $G-S$. Otherwise, we write that $P \nsim_{S} Q$. If the context is clear, we write $P \sim Q$ and $P \nsim Q$. Note that, in particular, if $V(P) \subseteq S$, then $P \nsim_{S} Q$ for every path $Q$. If $P \sim Q$ and $Q \sim R$, then we can abbreviate it by $P \sim Q \sim R$. Analogous abbreviations are given by $P \sim Q \nsim R$ and $P \nsim Q \sim R$. Given a cycle $C$ that 3 -crosses $S$ at $\{a, b, c\}$, we say that $a$ breaks $C$ if $C_{a b} \nsim_{S} C_{a c}$. If the context is clear, we also say that $a$ is a $C$-breaking vertex or that $a$ is a breaking vertex (Figures 2.10(a) and 2.10(c)).

A path (cycle) in a graph $G$ is called a longest path (cycle) if it has maximum length over all paths (cycles) in $G$. Let $S \subseteq V(G)$ and $P$ be a longest path (cycle) in $G$. We say 


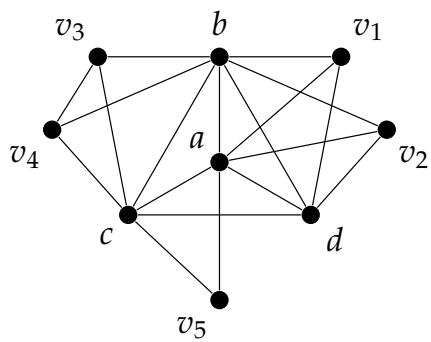

(a)

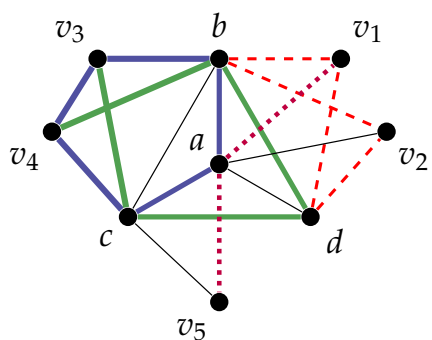

(b)

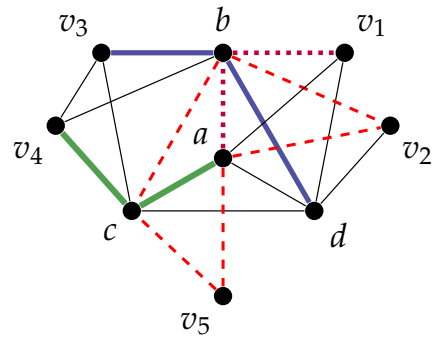

(c)

Figure 2.10: (a) A graph $G$ with $S=\{a, b, c, d\}$. (b) Consider paths $P_{1}=v_{1} a v_{5}$ and $P_{2}=v_{3} c d b v_{4}$, and cycles $C_{1}=v_{1} b v_{2} d v_{1}$ and $C_{2}=v_{3} v_{4} c a b v_{3}$. Then $P_{1}$ and $C_{1}$ cross $S$, and $P_{2}$ and $C_{2}$ are fenced by $S$. Moreover, $P_{1} 1$-crosses $S, P_{2}$ is 3 -fenced by $S, C_{1} 2$-crosses $S$ and $C_{2}$ is 3 -fenced by $S$. (Also note that path $c d$ and cycle $a b d a$ are fenced by $S$.) Paths $P_{2}$ and $v_{1} b c d v_{2}$ are $S$-equivalent. Cycles $C_{2}$ and $v_{1} b c v_{5} a v_{1}$ are $S$-equivalent. (c) Consider paths $P_{1}=v_{3} b d, P_{2}=v_{4} c a, P_{3}=v_{1} b a$ and cycle $C=a v_{2} b c v_{5} a$. Then $P_{1} \sim_{S} P_{2}, P_{1} \nsim_{S} P_{3}, P_{2} \nsim_{S} P_{3}, C_{a b} \nsim_{S} C_{a c}, C_{a b} \nsim_{S} C_{b c}$ and $C_{b c} \nsim_{S} C_{a c}$. Hence, $a$ breaks $C$.

that $P$ is an attractor for $S$ if $P$ is fenced by $S$ and all $S$-equivalent longest paths (cycles) are also fenced by $S$. We say that $P$ is a $k$-attractor if $|S \cap V(P)|=k$. In this case, we also say that $S$ has a $k$-attractor.

\subsection{Branches}

Let $G$ be a graph and $(T, \mathcal{V})$ be a tree decomposition of $G$. Given two different nodes $t, t^{\prime} \in V(T)$, we denote by $\operatorname{Branch}_{t}\left(t^{\prime}\right)$ the component of $T-t$ where $t^{\prime}$ lies. We say that such component is a branch of $T$ at $t$ and that the components of $T-t$ are the branches of $T$ at $t$ [Hei13]. Similarly, for a vertex $v \notin V_{t}$, it is denoted by $\operatorname{Branch}_{t}(v)$ the branch $\operatorname{Branch}_{t}\left(t^{\prime}\right)$ of $T$ at $t$ such that $v \in V_{t^{\prime}}$. In that case, we also say that $v \in \operatorname{Branch}_{t}\left(t^{\prime}\right)$ or that $v$ is in $\operatorname{Branch}_{t}\left(t^{\prime}\right)$.

Let $t \in V(T)$. Let $P$ be a path (cycle) in $G$ fenced by $V_{t}$. If $V(P) \subseteq V_{t}$, we say that $\operatorname{Branch}_{t}(P)=\varnothing$, that is, a subtree of $T$ with empty set or nodes and empty set of edges. Note that, in this case, $\operatorname{Branch}_{t}(P)$ is different from any branch of $T$ at $t$. Otherwise, we say that $\operatorname{Branch}_{t}(P)=\operatorname{Branch}_{t}(v)$, where $v \in V(P) \backslash V_{t}$. Also, we say that $P \in \operatorname{Branch}_{t}\left(t^{\prime}\right)$ or that $P$ is in $\operatorname{Branch}_{t}\left(t^{\prime}\right)$.

Next we show some basic properties of branches. For all the following propositions, we fix a graph $G$ and a tree decomposition $(T, \mathcal{V})$ of $G$. Proposition 2.6 is used to justify that the previous definitions are coherent.

\section{Proposition 2.6.}

(a) Let $t \in V(T)$ and let $v \in V(G) \backslash V_{t}$. Let $t^{\prime}, t^{\prime \prime} \in V(T)$. If $v \in V_{t^{\prime}} \cap V_{t^{\prime \prime}}$, then $\operatorname{Branch}_{t}\left(t^{\prime}\right)=\operatorname{Branch}_{t}\left(t^{\prime \prime}\right)$.

(b) Let $u, v \in V(G)$, and let $t \in V(T)$. If $u, v \notin V_{t}$, and $u$ and $v$ are not separated by $V_{t}$, 
then $\operatorname{Branch}_{t}(u)=\operatorname{Branch}_{t}(v)$.

(c) Let $t \in V(T)$ and $P$ be a path (cycle) in $G$ fenced by $V_{t}$. For every two vertices $u, v \in$ $V(P) \backslash V_{t}, \operatorname{Branch}_{t}(u)=\operatorname{Branch}_{t}(v)$.

Proof. Items $(a)$ and $(b)$ are Lemmas 3.2.2 and 3.2.4 in [Hei13] respectively. For item $(c)$, note that, by definition of fenced, $V_{t}$ does not separate $u$ and $v$, so we can apply item $(b)$.

Proposition 2.7 appears in the book of Diestel [Die10].

Proposition 2.7 ([Die10, Lemma 12.3.1]). Let $t t^{\prime} \in E(T)$. Let $u, v \in V(G)$ be such that $u \notin V_{t}$ and $v \notin V_{t^{\prime}}$. If $u \in \operatorname{Branch}_{t}\left(t^{\prime}\right)$ and $v \in \operatorname{Branch}_{t^{\prime}}(t)$, then $V_{t} \cap V_{t^{\prime}}$ separates $u$ and $v$.

The next proposition is crucial for our results on chordal graphs and partial $k$-trees.

Proposition 2.8. Let $t \in V(T)$. Let $P$ be a path (cycle) fenced by $V_{t}$ such that $\operatorname{Branch}_{t}(P)=\operatorname{Branch}_{t}\left(t^{\prime}\right)$, where $t t^{\prime} \in E(T)$. If $P$ does not have edges in $G\left[V_{t}\right]$, then $V_{t} \cap V(P) \subseteq V_{t^{\prime}}$.

Proof. Suppose by contradiction that there exists a vertex $x \in\left(V_{t} \cap V(P)\right) \backslash V_{t^{\prime}}$. As $P$ is fenced by $V_{t}$ and $\operatorname{Branch}_{t}(P)=\operatorname{Branch}_{t}\left(t^{\prime}\right)$, we have $|P|>0$. Thus, there exists an edge $x y \in E(P)$. Since $P$ does not have edges in $G\left[V_{t}\right], y \notin V_{t}$. Moreover, $\operatorname{Branch}_{t}(y)=\operatorname{Branch}_{t}(P)=\operatorname{Branch}_{t}\left(t^{\prime}\right)$ and, as $x \in V_{t} \backslash V_{t^{\prime}}, \operatorname{Branch}_{t^{\prime}}(x)=\operatorname{Branch}_{t^{\prime}}(t)$. But this is a contradiction to Proposition 2.7. which states that $V_{t} \cap V_{t^{\prime}}$ separates $x \in \operatorname{Branch}_{t^{\prime}}(t)$ from $y \in \operatorname{Branch}_{t}\left(t^{\prime}\right)$.

\subsection{Proof Techniques for Longest Paths and Longest Cycles}

In this section we introduce the main idea for proving our results on chordal graphs and partial $k$-trees when addressing longest paths and cycles. We begin by showing a proof for the well-known Helly Property on trees (see [Hel23] and [Hor72]).

Lemma 2.9. Let $T$ be a tree. Let $\mathscr{C}$ be a set of pairwise vertex-intersecting subtrees of $T$. There exists a vertex $t \in V(T)$ such that every tree in $\mathscr{C}$ contains $t$.

Proof. We define a partial orientation $T^{\prime}$ of $T$ as follows: $t t^{\prime} \in E\left(T^{\prime}\right)$ if and only if there exists a tree $P \in \mathscr{C}$, that does not contain $t$, such that $V(P)$ and $t^{\prime}$ are in the same component of $T-t$. Suppose by contradiction that the lemma is false for $T$. Then every node in $T^{\prime}$ has outdegree at least one. Let $t t^{\prime}$ be the last arc of a maximal directed path in $T^{\prime}$. As $T$ is a tree, $t^{\prime} t$ is also an arc in $T^{\prime}$, which implies that there exist two trees $P$ and $Q$ in $\mathscr{C}$ such that $V(P)$ and $t^{\prime}$ are in the same component of $T-t$, and $V(Q)$ 
and $t$ are in the same component of $T-t^{\prime}$. But then $V(P) \cap V(Q)=\varnothing$, a contradiction (Figure 2.11).

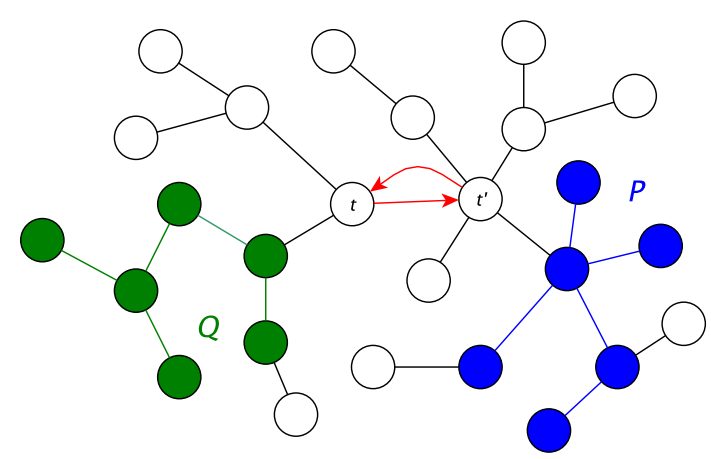

Figure 2.11: The subtrees $P$ and $Q$ that determined the orientation of $t t^{\prime}$ in Lemma 2.9

Observe that, as every two longest paths in a connected graph intersect each other, a direct corollary of Lemma 2.9 states that $\operatorname{lpt}(T)=1$ for every tree $T$. For the sake of completeness, we show the known property that every pair of longest paths intersect each other in connected graphs. We also show the analogous result for longest cycles. These two results (Propositions 2.10 and 2.12) are used many times throughout the text, we sometimes assume they are known properties and do not name them explicitly.

Proposition 2.10. Let $P$ and $Q$ be a pair of longest paths in a connected graph $G$. Then $V(P) \cap V(Q) \neq \varnothing$.

Proof. Suppose by contradiction that $V(P) \cap V(Q)=\varnothing$. Let $R$ be a path, internally disjoint from $P$ and $Q$, with one extreme in $P$ and the other in $Q$. Such a path exists because $G$ is connected ([BM08, Exercise 3.1.4]). Let $\{x\}=V(P) \cap V(R)$ and $\{y\}=$ $V(Q) \cap V(R)$. Let $P^{\prime}$ and $P^{\prime \prime}$ be the two $x$-tails of $P$. Let $Q^{\prime}$ and $Q^{\prime \prime}$ be the two $y$-tails of $Q$. Then $P^{\prime} \cdot R \cdot Q^{\prime}$ and $P^{\prime \prime} \cdot R \cdot Q^{\prime \prime}$ are both paths, one of them longer than $|P|$, a contradiction.

Corollary 2.11. In a tree, all longest paths contain a common vertex.

Proof. This follows from Lemma 2.9 and Proposition 2.10.

Proposition 2.12. Let $C$ and $D$ be a pair of longest cycles in a 2-connected graph $G$. Then $|V(C) \cap V(D)| \geq 2$.

Proof. Suppose by contradiction that $|V(C) \cap V(D)| \leq 1$. As $G$ is 2-connected, there exist two disjoint paths $R$ and $S$, both of them with one extreme in $C$, the other in $D$, and internally disjoint from both $C$ and $D$ ([BM08, Proposition 9.4]). Note 
that, when $|V(C) \cap V(D)|=1$, it can be the case that exactly one of $\{R, S\}$ has zero length. Let $\left\{x_{1}\right\}=V(C) \cap V(R)$ and $\left\{x_{2}\right\}=V(C) \cap V(S)$. Let $\left\{y_{1}\right\}=V(D) \cap V(R)$ and $\left\{y_{2}\right\}=V(D) \cap V(S)$. Let $C^{\prime}$ and $C^{\prime \prime}$ be the two $x_{1} x_{2}$-parts of $C$. Let $D^{\prime}$ and $D^{\prime \prime}$ be the two $y_{1} y_{2}$-parts of $D$. Then $C^{\prime} \cdot R \cdot D^{\prime} \cdot S$ and $C^{\prime \prime} \cdot R \cdot D^{\prime \prime} \cdot S$ are both cycles, one of them longer than $|C|$, a contradiction.

When considering chordal graphs and partial $k$-trees, our main technique is inspired on the idea of orienting the edges of the tree, used in Lemma 2.9. so we apply it to the corresponding tree decomposition of the graph. This is shown in Lemma 2.14. Before it, we show a useful property.

Proposition 2.13. Let $(T, \mathcal{V})$ be a tree decomposition of a graph $G$. If $P$ is a path (cycle) in $G$ fenced by $V_{t}$, for some $t \in V(T)$, then either $V(P) \subseteq V_{t}$ or there exists an edge $t t^{\prime} \in E(T)$ such that $\operatorname{Branch}_{t}(P)=\operatorname{Branch}_{t}\left(t^{\prime}\right)$.

Proof. If $V(P) \subseteq V_{t}$, then there is nothing to prove. Otherwise, let $u \in V(P) \backslash V_{t}$. As $u \notin V_{t}$, there exists a bag $V_{t^{\prime \prime}}$ that contains $u$. Clearly $t^{\prime \prime} \neq t$. Let $t^{\prime}$ be the neighbor of $t$ in $T$ such that $t^{\prime}$ is in the (unique) path from $t$ to $t^{\prime \prime}$ in $T$. Then $\operatorname{Branch}_{t}(P)=$ $\operatorname{Branch}_{t}(u)=\operatorname{Branch}_{t}\left(t^{\prime \prime}\right)=\operatorname{Branch}_{t}\left(t^{\prime}\right)$.

The next lemma is crucial for all proofs on chordal graphs and partial $k$-trees.

Lemma 2.14. Let $(T, \mathcal{V})$ be a tree decomposition of a graph $G$. For every node $t$, let $\mathscr{P}(t)$ be a set of paths (cycles) in $G$ fenced by $V_{t}$ but not contained in $G\left[V_{t}\right]$. If $\mathscr{P}(t) \neq \varnothing$ for every node $t \in V(T)$, then there exists an edge $t t^{\prime} \in E(T)$ and two paths (cycles) $P \in \mathscr{P}(t)$ and $Q \in \mathscr{P}\left(t^{\prime}\right)$ such that $\operatorname{Branch}_{t}(P)=\operatorname{Branch}_{t}\left(t^{\prime}\right)$ and $\operatorname{Branch}_{t^{\prime}}(Q)=\operatorname{Branch}_{t^{\prime}}(t)$.

Proof. We define a partial orientation $T^{\prime}$ of $T$ as follows: $t t^{\prime} \in E\left(T^{\prime}\right)$ if and only if $t t^{\prime} \in E(T)$ and there exists a path (cycle) $P \in \mathscr{P}(t) \operatorname{such}$ that $\operatorname{Branch}_{t}(P)=\operatorname{Branch}_{t}\left(t^{\prime}\right)$. For every $t \in V(T)$, as $\mathscr{P}(t) \neq \varnothing$, there exists a path (cycle) $P$ fenced by $V_{t}$ and such that $V(P) \nsubseteq V_{t}$. Thus, by Proposition 2.13, there exists a neighbor $t^{\prime}$ of $t$ in $T$ such that $\operatorname{Branch}_{t}(P)=\operatorname{Branch}_{t}\left(t^{\prime}\right)$. Hence every node in $T^{\prime}$ has outdegree at least one. Let $t t^{\prime}$ be the last arc of a maximal directed path in $T^{\prime}$. As $T$ is a tree, $t^{\prime} t$ is also an arc in $T^{\prime}$, which implies that there exist two paths (cycles) $P \in \mathscr{P}(t)$ and $Q \in \mathscr{P}\left(t^{\prime}\right)$ such that $\operatorname{Branch}_{t}(P)=\operatorname{Branch}_{t}\left(t^{\prime}\right)$ and $\operatorname{Branch}_{t^{\prime}}(Q)=\operatorname{Branch}_{t^{\prime}}(t)$.

We use Lemma 2.14 repeatedly to obtain results for longest paths and longest cycles in chordal graphs and partial $k$-trees. It is not the first time this idea is used. According to Diestel [Die10], given a graph $G$, a bramble in $G$ is a set $\mathscr{C}$ with the following properties: (i) every element $H \in \mathscr{C}$ is a subset $H \subseteq V(G)$ such that $G[H]$ is connected, and (ii) every pair $H_{1}, H_{2} \in \mathscr{C}$ is such that either $H_{1} \cap H_{2} \neq \varnothing$ or there exists an edge $u v \in E(G)$ 
with $u \in H_{1}$ and $v \in H_{2}$. The order of $\mathscr{C}$ is the cardinality of a minimum set of vertices that intersects every set in $\mathscr{C}$.

The following theorem is due to Seymour and Thomas, and appears in the book of Diestel.

Theorem 2.15 ([Die10, Theorem 12.3.9]). Let $k \geq 0$ be an integer. A graph has treewidth at least $k$ if and only if it contains a bramble of order larger than $k$.

We can derive the following corollary, which was also observed by Rautenbach and Sereni [RS14, Proposition 2.6] but only for longest paths.

Corollary 2.16. Let $G$ be a connected (2-connected) graph. Then $\operatorname{lpt}(G) \leq \operatorname{tw}(G)+1$ $(\operatorname{lct}(G) \leq \operatorname{tw}(G)+1)$.

Proof. Let $\mathscr{C}=\{V(P): P$ is a longest path (cycle) in $G\}$. By Proposition 2.10 (Proposition 2.12), every pair of elements in $\mathscr{C}$ intersect each other, so $\mathscr{C}$ is a bramble in $G$. By Theorem 2.15, $\mathscr{C}$ has order at most $\operatorname{tw}(G)+1$, that is, $\operatorname{lpt}(G) \leq \operatorname{tw}(G)+1$ $(\operatorname{lct}(G) \leq \operatorname{tw}(G)+1)$.

By Proposition 2.3. $\operatorname{tw}(G)=\omega(G)-1$ when $G$ is chordal. Hence, we can easily conclude the following corollary, which was also observed by Balister et al. [BGLS04], but only for longest paths.

Corollary 2.17. Let $G$ be a connected (2-connected) chordal graph. Then $\operatorname{lpt}(G) \leq \omega(G)$ $(\operatorname{lct}(G) \leq \omega(G))$.

Proof. It follows from Corollary 2.16 and Proposition 2.3 .

One of the main tasks in this thesis is to improve the bounds of Corollary 2.16 and Corollary 2.17. For that, as we said, we mimic the idea for trees with the help of Lemma 2.14. First, we focus on an arbitrary bag $V_{t}$ of the tree decomposition (if the graph is chordal, we focus on a maximal clique). Then, unless the graph is complete, we try to find a longest path (cycle) fenced by $V_{t}$ that satisfies a particular property, which will make our set $\mathscr{P}(t)$ nonempty for every $t \in V(T)$, to finally apply our Lemma 2.14 .

The main difficulty in every case is to prove that such a path (cycle) exists. But we will see that it is very unlikely that a set of longest paths (cycles) that cross a particular bag, and intersect the bag in a small quantity of vertices, can coexist. Hence, we will obtain at least one fenced path (cycle), for each node of the tree decomposition, in order to a partial orientation of the tree. In the next chapters (Chapter 3 and Chapter 4), we will see the details of how this path (cycle) is obtained.

To finish this section, we give a proof of a result that is used directly in Theorem 4.22 . 
Proposition 2.18. Let $G$ be a 2-connected graph. Let $L(G)$ be the length of a longest cycle in $G$. If $L(G) \leq 4$, then $\operatorname{lct}(G)=1$.

Proof. Suppose that $L:=L(G)=3$. Let abca be a longest cycle in $G$. Suppose by contradiction that $\operatorname{lct}(G)>1$. Then, there exists a longest cycle $C$ that does not contain $a$. By Proposition 2.12, as $G$ is 2-connected, $b, c \in V(C)$. Let $C^{\prime}$ and $C^{\prime \prime}$ be the two $b c$-parts of $C$, with $\left|C^{\prime}\right| \geq\left|C^{\prime \prime}\right|$. As $L=3$, we have $\left|C^{\prime}\right|=2$. But then $b a \cdot a c \cdot C^{\prime}$ is a cycle of length 4 , a contradiction.

So we may assume that $L=4$. Let $C=a b c d a$ be a longest cycle in $G$. We show that $\{a, b\}$ is a transversal of $G$. Suppose by contradiction that this is not the case. Let $D$ be a longest cycle in $G$ such that $a, b \notin V(D)$. As $G$ is 2-connected, $c, d \in V(D)$. Let $D^{\prime}$ and $D^{\prime \prime}$ be the two $c d$-parts of $D$, with $\left|D^{\prime}\right| \geq\left|D^{\prime \prime}\right|$. As $L=4$, we have $\left|D^{\prime}\right| \geq 2$. But then $a b \cdot b c \cdot D^{\prime} \cdot d a$ is a cycle of length at least 5 , a contradiction. Thus, $\{a, b\}$ is a transversal of $G$. Analogously, $\{b, c\},\{c, d\}$ and $\{a, d\}$ are also transversals of $G$.

Suppose by contradiction that $\operatorname{lct}(G)>1$. Then, there exists a longest cycle $D$ that does not contain $a$. As both $\{a, b\}$ and $\{a, d\}$ are transversals of $G$, then $b, d \in V(D)$. Let $D^{\prime}$ and $D^{\prime \prime}$ be the two $b d$-parts of $D$. As these two parts are internally disjoint, we may assume that $c \notin V\left(D^{\prime}\right)$. Also, observe that $\left|D^{\prime}\right| \geq 2$, otherwise $a b \cdot D^{\prime \prime} \cdot d a$ is a cycle of length five, a contradiction. Moreover, $\left|D^{\prime}\right|=2$, otherwise $a b \cdot D^{\prime} \cdot d a$ is a cycle of length five, a contradiction. As $\operatorname{lct}(G)>1$, there exists a longest cycle $F$ that does not contain $b$. By a similar analysis, with $b$ instead of $a$, we may assume that $F$ has an $a c$ part $F^{\prime}$ that does not contain $d$ and $\left|F^{\prime}\right|=2$. If $D^{\prime}$ and $F^{\prime}$ are disjoint, then $a b \cdot D^{\prime} \cdot d c \cdot F^{\prime}$ is a cycle of length six, a contradiction. Hence, there exists a vertex $x \in V\left(D^{\prime}\right) \cap V\left(F^{\prime}\right)$. In this case, $a b x c d a$ is a cycle of length five, again a contradiction. 


\section{Chapter 3}

\section{Transversals of Longest Paths}

In this chapter we address transversals of longest paths in some graph classes. Recall that lpt is the minimum cardinality of a transversal of longest paths. We prove the following:

- $\operatorname{lpt}(G) \leq \max \{1, \omega(G)-2\}$ for every connected chordal graph $G$ (Section 3.1).

- $\operatorname{lpt}(G) \leq k$ for every connected partial $k$-tree $G$ (Section 3.2).

- $\operatorname{lpt}(G)=1$ for every connected full substar graph $G$ (Section 3.3).

- $\operatorname{lpt}(G)=1$ for every connected bipartite permutation graph $G$ (Section 3.4).

Throughout this chapter, we use $L=L(G)$ for the length of a longest path in $G$. To recall the basic definitions on longest paths, see Section 2.3 .

\subsection{Chordal Graphs}

In this section, we prove that $\operatorname{lpt}(G) \leq \max \{1, \omega(G)-2\}$ for every connected chordal graph $G$ (Theorem 3.5). Our main idea is to generalize Lemma 2.9 with the help of Lemma 2.14 (see Section 2.5). This idea has been successfully used for 2-trees [dFMW13]. To achieve this for chordal graphs, we use a corresponding clique tree of the graph (that is, a tree decomposition as in Proposition 2.5). First, we focus on an arbitrary bag (a maximal clique) of such a tree decomposition and analyze the behaviour of longest paths whose extremes are separated or not by the clique (Lemma 3.1 and Lemma 3.2). Then we use these results to prove that there exists a longest path that is fenced by that clique and satisfies some special properties (Lemma 3.4). Finally, with the help of this longest path, we use Lemma 2.14 to obtain our final contradiction (Theorem 3.5.

We start by analyzing the behaviour of longest paths, whose extremes are not separated, in a particular clique. The next lemmas are valid for any graph (not necessarily chordal). 
Lemma 3.1. Let $G$ be a graph with a clique K. Let $\mathscr{C}$ be the set of all longest paths in $G$ that 2 -cross $K$ and whose extremes are not separated by $K$. For every three paths in $\mathscr{C}$ there are two of them that are equivalent.

Proof. Suppose by contradiction that there are (at least) three longest paths $P, Q$, and $R$ in $\mathscr{C}$ that are pairwise not $K$-equivalent. Say $V(P) \cap K=\{a, b\}, V(Q) \cap K=\{c, d\}$, and $V(R) \cap K=\{e, f\}$, where $\{a, b\},\{c, d\}$, and $\{e, f\}$ are pairwise distinct but not necessarily pairwise disjoint. We may assume that either $\{a, b\} \cap\{c, d\}=\varnothing$ or $\{a, b\} \cap$ $\{c, d\}=\{b\}=\{d\}$. If $\tilde{P}$ is internally disjoint from $Q_{c}$ and from $Q_{d}$, and $\tilde{Q}$ is internally disjoint from $P_{a}$ and from $P_{b}$, then $P_{a} \cdot a c \cdot \tilde{Q} \cdot d b \cdot P_{b}$ and $Q_{c} \cdot c a \cdot \tilde{P} \cdot b d \cdot Q_{d}$ are paths whose lengths sum more than $2 L$, a contradiction, as at least one of them would have length greater than $L$ (Figure 3.1(a)). Observe also that, as $P$ is a longest path, it has no extremes in $K$, so both $P_{a}$ and $P_{b}$ have vertices not in $K$ and, as $P \in \mathscr{C}$, we have $P_{a} \sim P_{b}$. Analogously, $Q_{c} \sim Q_{d}$ and $R_{e} \sim R_{f}$.

So,

$$
\text { either } \tilde{P} \sim Q_{c} \sim Q_{d} \text { or } \tilde{Q} \sim P_{a} \sim P_{b} \text {. }
$$

Applying the same reasoning to paths $P$ and $R$, and to paths $Q$ and $R$, we conclude that

$$
\text { either } \tilde{P} \sim R_{e} \sim R_{f} \text { or } \tilde{R} \sim P_{a} \sim P_{b}
$$

and that

$$
\text { either } \tilde{Q} \sim R_{e} \sim R_{f} \text { or } \tilde{R} \sim Q_{c} \sim Q_{d} \text {. }
$$

Also, as $P, Q$, and $R$ cross $K$, from (3.1), (3.2), and (3.3), we have that

$$
\tilde{P} \nsim \tilde{Q}, \tilde{P} \nsim \tilde{R} \text {, and } \tilde{Q} \nsim \tilde{R} \text {. }
$$

Without loss of generality, we may assume that $\tilde{P} \sim Q_{c} \sim Q_{d}$ (Otherwise, interchange $P$ with $Q$, and $\{a, b\}$ with $\{c, d\}$.) Now, if $\tilde{P} \sim R_{e}$, then $\tilde{Q} \nsim R_{e}$ by $(3.4)$, and thus $\tilde{R} \sim Q_{c}$ by (3.3). But then $\tilde{R} \sim \tilde{P}$, and we contradict 3.4. Hence, $\tilde{P} \nsim R_{e} \sim R_{f}$, and $\tilde{R} \sim P_{a}$ by 3.2. Similarly, one can deduce that $\tilde{R} \nsim Q_{c} \sim Q_{d}$. Thus, $\tilde{Q} \sim R_{e}$ by 3.3, and, again, we can deduce that $\tilde{Q} \nsim P_{a} \sim P_{b}$ (Figure 3.1 (b). We conclude that

$$
P_{a} \sim P_{b} \nsim \tilde{Q}, Q_{c} \sim Q_{d} \nsim \tilde{R} \text { and } R_{e} \sim R_{f} \nsim \tilde{P} .
$$

We may assume that either $\{a, b\} \cap\{e, f\}=\varnothing$ or $\{a, b\} \cap\{e, f\}=\{b\}=\{f\}$, and that either $\{c, d\} \cap\{e, f\}=\varnothing$ or $\{c, d\} \cap\{e, f\}=\{d\}=\{f\}$ (the proofs for other cases are analogous). Therefore, by (3.5), we have three paths, $P_{a} \cdot a c \cdot \widetilde{Q} \cdot d b \cdot P_{b}$, $Q_{c} \cdot c e \cdot \widetilde{R} \cdot f d \cdot Q_{d}$, and $R_{e} \cdot e a \cdot \widetilde{P} \cdot b f \cdot R_{f}$, whose lengths sum more than $3 L$, which leads to a contradiction. 


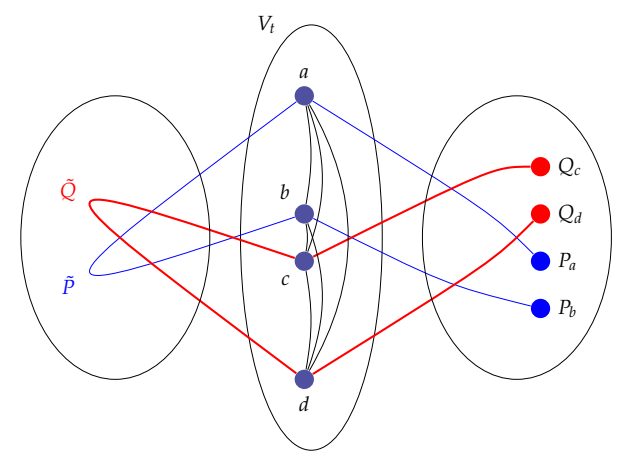

(a)

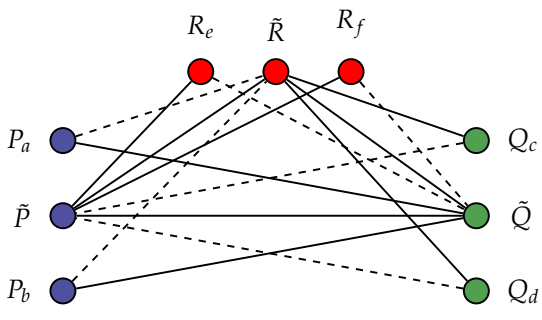

(b)

Figure 3.1: (a) Paths $P$ and $Q$ from the proof of Lemma 3.1. (b) Paths $P, Q$, and $R$, and a tripartite graph representing the interaction between their parts. The vertex set of the graph has three vertices for each of the paths, one for each part. The edge set contains two types of edges: straight edges connect parts that are in different components of $G-K$ and dashed edges connect parts that are in the same component of $G-K$. When the interaction between two parts is not determined, we omit the edge between them.

The previous lemma treats how longest paths whose extremes are not separated by a clique behave. The next lemma treats the case in which the longest paths have their extremes separated by the clique. Observe that in both cases we are considering only longest paths that cross the clique and intersect it at most twice.

Lemma 3.2. Let $G$ be a graph with a clique $K$. Let $\mathscr{C}$ be the set of all longest paths in $G$ that intersect $K$ at most twice and whose extremes are separated by $K$. Every two elements of $\mathscr{C}$ have a common vertex of $K$.

Proof. Let $P$ and $Q$ be two arbitrary paths in $\mathscr{C}$. Suppose by contradiction that $V(P) \cap V(Q) \cap K=\varnothing$. As $K$ separates the extremes of $P$, path $P$ crosses $K$ and therefore $P$ either 1-intersects or 2-intersects $K$. To address these two possibilities at once, let $x$ and $y$ be such that $P$ intersects $K$ at $x$ and $y$, with $x \neq y$ if $P$ 2-intersects $K$. Also, if $x=y$, then let $\widetilde{P}$ be the path consisting of only vertex $x$, and we abuse notation and consider that $P_{x}$ and $P_{y}$ are different $x$-tails of $P$. Let $w$ and $z$, and possibly $Q_{w}, Q_{z}$, and $\widetilde{Q}$, be defined similarly for $Q$.

As $K$ separates the extremes of both $P$ and $Q$, we may assume without loss of generality that $P_{x} \nsim Q_{w}$ and $P_{y} \nsim Q_{z}$. (Otherwise interchange $w$ and $z$.) Observe also that either $\widetilde{P} \nsim Q_{w}$ or $\widetilde{P} \nsim Q_{z}$. Without loss of generality, assume that $\widetilde{P} \nsim Q_{w}$. (Otherwise interchange $x$ and $y$, and $w$ and $z$ simultaneously.)

Note that either $\widetilde{Q} \nsim P_{x}$ or $\widetilde{Q} \nsim P_{y}$. Suppose that $\widetilde{Q} \nsim P_{y}$. (See a representation of the interactions between the parts of $P$ and $Q$ in Figure 3.2(a).) Then, one of the paths $P_{x} \cdot \widetilde{P} \cdot y w \cdot Q_{w}$ or $P_{y} \cdot y w \cdot \widetilde{Q} \cdot Q_{z}$ is longer than $L$, a contradiction. So, we may assume that $\widetilde{Q} \sim P_{y}$ and that $\widetilde{Q} \nsim P_{x}$. 
If $\widetilde{P} \nsim \widetilde{Q}$ (Figure 3.2(b)), then one of $P_{x} \cdot \widetilde{P} \cdot y z \cdot \widetilde{Q} \cdot Q_{w}$ or $P_{y} \cdot y z \cdot Q_{z}$ is longer than $L$, a contradiction. If $\widetilde{P} \sim \widetilde{Q}$, then $\widetilde{P} \sim \widetilde{Q} \sim P_{y} \nsim Q_{z}$. Hence $Q_{z} \nsim \widetilde{P}$ (Figure 3.2.(c)). Thus, one of the paths $P_{x} \cdot x z \cdot \widetilde{Q} \cdot Q_{w}$ or $P_{y} \cdot \widetilde{P} \cdot x z \cdot Q_{z}$ is longer than $L$, a contradiction.

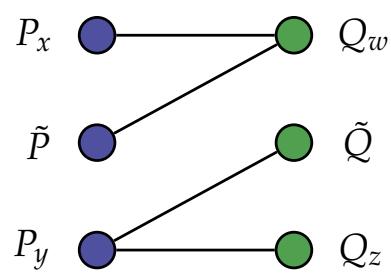

(a)

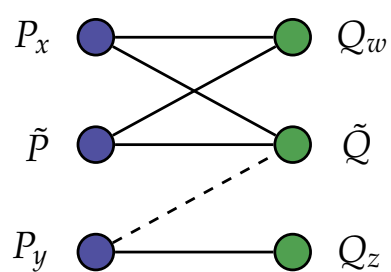

(b)

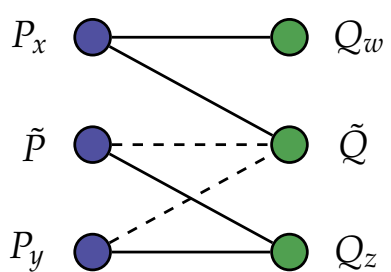

(c)

Figure 3.2: Each bipartite graph represents the situation of paths $P$ and $Q$ in one of the cases of the proof of Lemma 3.2. Each side of the bipartition has three vertices that represent the parts of each path. There is a straight edge in the graph if the two corresponding vertices are in different components of $G-K$ and a dashed edge if they are in the same component.

The next lemma synthesizes the two previous lemmas when the graph is chordal and connected. It says that, for every clique, when there is no transversal totally contained in it, we would have a longest path that is an attractor for the clique. Recall that a longest path $P$ is an attractor for a clique $K$ if $P$ is fenced by $K$ and all $K$-equivalent longest paths are also fenced by $K$ (Section 2.3).

For the proof of the lemma, we use the next result on connected graphs with treewidth at most two.

Proposition $3.3\left(\left[\mathrm{CEF}^{+} 17\right]\right)$. If $G$ is a connected graph with $\operatorname{tw}(G) \leq 2$, then $\operatorname{lpt}(G)=1$.

Lemma 3.4. Let $G$ be a connected chordal graph such that $\operatorname{lpt}(G)>\max \{1, \omega(G)-2\}$. Let $k$ be an integer with $k \geq 2$. For each clique of cardinality $k$ in $G$, there exists an $\ell$-attractor, with $\ell \leq \min \{2, k-2\}$.

Proof. If $\omega(G) \leq 3$, then $\operatorname{tw}(G) \leq 2$ by Proposition 2.3, so, by Proposition 3.3, we have $\operatorname{lpt}(G)=1=\max \{1, \omega(G)-2\}$, and the proof follows. Therefore, $\omega(G) \geq 4$ and $\max \{1, \omega(G)-2\}=\omega(G)-2$. Let $K$ be a clique in $G$ and $k \geq 2$ be its cardinality. If $k<\operatorname{lpt}(G)$, then there is a longest path that does not intersect $K$. Such path is a 0 -attractor for $K$. Hence, there is an $\ell$-attractor, with $\ell \leq \min \{2, k-2\}$ for $K$. Thus, $k \geq \operatorname{lpt}(G)$. Since $\operatorname{lpt}(G)>\max \{1, \omega(G)-2\}=\omega(G)-2$, we have $k \in\{\omega(G)-1, \omega(G)\}$.

If $k=\omega(G)-1$, then, as $\operatorname{lpt}(G)>\omega(G)-2$, for every vertex in $K$, there exists a longest path that 1 -intersects $K$ at that vertex. Suppose by contradiction that none of these paths is a 1-attractor for $K$. Then, for every vertex in $K$, there exists a longest path that crosses $K$ at that vertex. As $k=\omega(G)-1 \geq 3$, two of these paths do not 
intersect each other at $K$, a contradiction to Lemma 3.2. Thus, there is a 1-attractor for $K$. As $k \geq 3$, there is an $\ell$-attractor, with $\ell \leq \min \{2, k-2\}$ for $K$ and we are done. Hence, we may assume that $k=\omega(G) \geq 4$.

Case 1: There exists a longest path that 1-intersects $K$.

Let $v$ be the corresponding vertex. Suppose by contradiction that $K$ has no $\ell$-attractor, with $\ell \leq 2$. Then, there exists a longest path $P$ that crosses $K$ at $v$. As $\operatorname{lpt}(G)>k-2$, for every $(k-2)$-clique in $K$ containing $v$, there exists a longest path that does not contain any vertex in that clique. If any of these longest paths 1 -intersects $K$ at a vertex $w$, then, as $K$ has no 1-attractor, there exists a longest path that crosses $K$ at $w$, contradicting Lemma 3.2 .

Hence, for every edge in $K$ not incident to $v$, there exists a longest path that 2intersects $K$ at the ends of that edge. Again, by Lemma 3.2. as $P$ crosses $K$ at $v$, none of these paths has its extremes separated by $K$. As $k \geq 4$, there are at least three such paths. By Lemma 3.1. there exists an edge $e$ of $K-v$ such that no longest path 2-crosses $K$ at the ends of $e$. Moreover, we know that there exists a longest path that 2-intersects $K$ at the ends of $e$. Hence, such path is a 2-attractor for $K$.

Case 2: Every longest path intersects $K$ at least twice.

$\operatorname{As} \operatorname{lpt}(G)>k-2$, for every edge $e$ of $K$, there exists a longest path that 2-intersects $K$ at the ends of $e$. As $k \geq 4$, there are at least six not $K$-equivalent longest paths that 2 -intersect $K$. Suppose by contradiction that there is no $\ell$-attractor for $K$, with $\ell \leq 2$. Then, for every edge of $K$, there exists a longest path that 2-crosses $K$ at the ends of that edge. Let $A$ be a subgraph of $K$ with four vertices. By Lemma 3.1, at least four of the corresponding longest paths in $A$ have their extremes separated by $K$. As at least two of the corresponding edges of $A$ are disjoint, by Lemma 3.2, we have a contradiction.

This concludes the proof of the lemma.

We can finally prove our main result.

Theorem 3.5. For every connected chordal graph $G, \operatorname{lpt}(G) \leq \max \{1, \omega(G)-2\}$.

Proof. Let $(T, \mathcal{V})$ be a clique tree of $G$, which exists by Proposition 2.5. If one of such cliques have cardinality one, then $|V(G)|=1$ and we are done. Thus, every clique in $\mathcal{V}$ has cardinality at least two. For every $t \in V(T)$, let $\mathscr{P}(t)$ be the set of longest paths in $G$ which are $\ell$-attractors for $V_{t}$, with $\ell \leq \min \left\{2,\left|V_{t}\right|-2\right\}$. Suppose by contradiction that $\operatorname{lpt}(G)>\max \{1, \omega(G)-2\}$. Then, $\mathscr{P}(t) \neq \varnothing$ for every $t \in V(T)$ by Lemma 3.4. As every $P \in \mathscr{P}(t)$ is a longest path, $V(P) \nsubseteq V_{t}$. Thus, by Lemma 2.14, there exists an edge $t t^{\prime} \in E(T)$ and two paths $P \in \mathscr{P}(t)$ and $Q \in \mathscr{P}\left(t^{\prime}\right)$ 
such that $\operatorname{Branch}_{t}(P)=\operatorname{Branch}_{t}\left(t^{\prime}\right), \operatorname{Branch}_{t^{\prime}}(Q)=\operatorname{Branch}_{t^{\prime}}(t), P$ is an $\ell$-attractor for $V_{t}$, with $\ell \leq \min \left\{2,\left|V_{t}\right|-2\right\}$, and $Q$ is an $\ell^{\prime}$-attractor for $V_{t^{\prime}}$, with $\ell^{\prime} \leq \min \left\{2,\left|V_{t^{\prime}}\right|-2\right\}$.

Let $u \in V_{t} \backslash V_{t^{\prime}}$. Suppose for a moment that $u \in V(P)$. Let $u v \in E(P)$. By Proposition 2.7, $\operatorname{Branch}_{t}(v) \neq \operatorname{Branch}_{t}\left(t^{\prime}\right)$, so $v \in V_{t}$. This implies that $u v$ is an edge in $V_{t}$ and, as $V_{t}$ is a clique, $P$ contains all vertices of $V_{t}$, a contradiction to the fact that $\ell \leq \min \left\{2,\left|V_{t}\right|-2\right\}$. Thus, $\left(V_{t} \backslash V_{t^{\prime}}\right) \cap V(P)=\varnothing$ and $\left(V_{t^{\prime}} \backslash V_{t}\right) \cap V(Q)=\varnothing$, which implies, by Proposition 2.7, that $V_{t} \cap V_{t^{\prime}}$ separates a vertex in $V(P) \backslash V_{t}$ from a vertex in $V(Q) \backslash V_{t^{\prime}}$.

Suppose for a moment that $\left|V_{t} \cap V_{t^{\prime}}\right| \leq \omega(G)-2$. As $\operatorname{lpt}(G)>\max \{1, \omega(G)-2\}$, there exists a longest path $R$ that does not contain any vertex of $V_{t} \cap V_{t^{\prime}}$. This leads to a contradiction, because, as $G$ is connected, $R$ must intersect a vertex in $V(P) \backslash V_{t}$ and a vertex in $V(Q) \backslash V_{t^{\prime}}$. Hence $\left|V_{t} \cap V_{t^{\prime}}\right| \geq \omega(G)-1$. Moreover, as both $V_{t}$ and $V_{t^{\prime}}$ are maximal (and different), we conclude that $\left|V_{t}\right|=\left|V_{t^{\prime}}\right|=\omega(G)$ and $\left|V_{t} \cap V_{t^{\prime}}\right|=\omega(G)-1$. Let $\{u\}=V_{t} \backslash V_{t^{\prime}}$ and $\{w\}=V_{t^{\prime}} \backslash V_{t}$.

Since $G$ is connected, $V(P) \cap V(Q) \neq \varnothing$, therefore $\ell \geq 1$ and $\ell^{\prime} \geq 1$. Indeed, suppose for example that $\ell=0$. Then, as $V(Q) \cap\left(V_{t^{\prime}} \backslash V_{t}\right)=\varnothing$, we would have that $P$ and $Q$ do not intersect, a contradiction. First suppose that $P$ 1-intersects $V_{t}$ at a vertex $a$. That is, $P$ is a 1-attractor for $V_{t}$. So $V(P) \cap V(Q)=\{a\}$. That is, $P$ and $Q$ only intersect each other at $a$, which implies that $a$ divides both longest paths in half. Let $P^{\prime}$ and $P^{\prime \prime}$ be the two $a$-tails of $P$, and let $Q^{\prime}$ and $Q^{\prime \prime}$ be the two $a$-tails of $Q$. As $P 1$-intersects $V_{t}$, we may assume without loss of generality that $w \notin P^{\prime}$.

Suppose that $Q$ also 1-intersects $V_{t^{\prime}}$. Then, we may assume without loss of generality that $u \notin Q^{\prime}$, which means that $P^{\prime} \cdot Q^{\prime}$ is a longest path that crosses $V_{t}$ at $\{a\}$, a contradiction to the fact that $P$ is a 1-attractor for $V_{t}$ and intersects $V_{t}$ at $\{a\}$ (Figure 3.3(a)). Now suppose that $Q$ 2-intersects $V_{t^{\prime}}$ at $\{a, b\}$. Note that either $Q_{a}=Q^{\prime}$ or $Q_{a}=Q^{\prime \prime}$. If $u \notin Q_{a}$ then $P^{\prime} \cdot Q_{a}$ is a longest path that crosses $V_{t}$ at $\{a\}$, again a contradiction. Hence, $u \in Q_{a}$. But then $P^{\prime} \cdot \tilde{Q} \cdot Q_{b}$ is a longest path that 2-intersects $V_{t^{\prime}}$ and crosses $V_{t^{\prime}}$, a contradiction to the fact that $Q$ is a 2-attractor for $V_{t}$ and intersects $V_{t}$ at $\{a, b\}$. Therefore, $P$ 2-intersects $V_{t}$.

By a similar reasoning, we conclude that $Q$ 2-intersects $V_{t^{\prime}}$. Suppose that $P 2-$ intersects $V_{t}$ at the ends of edge $a b$. First suppose that $Q$ also 2-intersects $V_{t^{\prime}}$ at the same vertices. Then, $\left|P_{a}\right|=\left|Q_{a}\right|,\left|P_{b}\right|=\left|Q_{b}\right|$, and $|\tilde{P}|=|\tilde{Q}|$. If $u \notin Q_{a}$, then $P_{b} \cdot \tilde{P} \cdot Q_{a}$ is a longest path that 2-intersects $V_{t}$ and crosses $V_{t}$, a contradiction to the fact that $P$ is a 2-attractor for $V_{t}$ and intersects $V_{t}$ at $\{a, b\}$ (Figure 3.3 (b)). Hence, $u \in Q_{a}$ and $u \notin \tilde{Q}$. Thus $P_{a} \cdot \tilde{Q} \cdot P_{b}$ is a longest path that 2-intersects $V_{t}$ and crosses $V_{t}$, again a contradiction. Hence, we may assume that $Q$ 2-intersects $V_{t^{\prime}}$ at the ends of an edge $b c$ with $c \neq a$. Therefore $P_{a} \cdot a c \cdot \tilde{Q} \cdot P_{b}$ and $Q_{c} \cdot c a \cdot \tilde{P} \cdot Q_{b}$ are paths, because $a \neq u$ and $c \neq w$, and one of them is longer than $L$, yielding the final contradiction (Figure 3.3. (c)). 


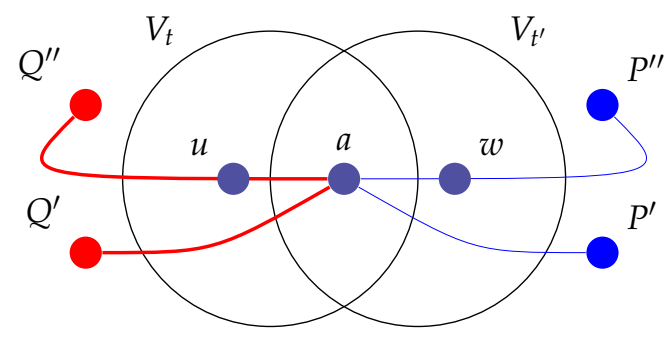

(a)

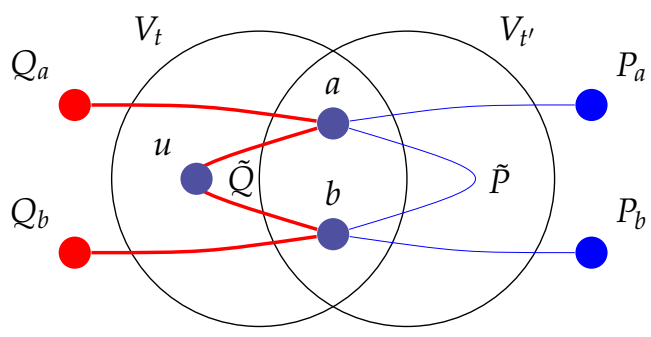

(b)

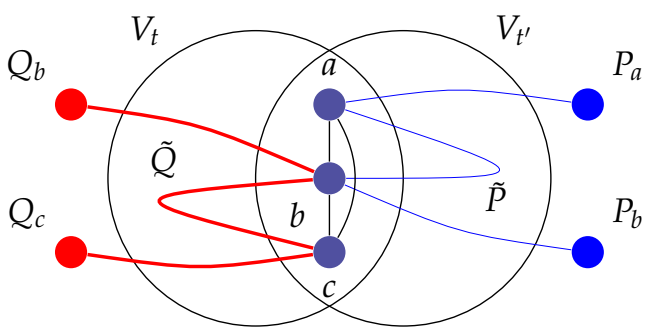

(c)

Figure 3.3: Cases in the proof of Theorem 3.5

The previous theorem implies the following results.

Corollary 3.6. If $G$ is a $k$-tree, with $k>1$, then $\operatorname{lpt}(G) \leq k-1$.

Corollary 3.7. If $G$ is a 3-tree or a connected chordal planar graph, then $\operatorname{lpt}(G) \leq 2$.

\subsection{Partial $k$-Trees}

The aim of this section is to prove that $\operatorname{lpt}(G) \leq k$ for any connected partial $k$-tree $G$ (Theorem 3.10). Recall that, by Proposition 2.2, partial $k$-trees are exactly those graphs with treewidth at most $k$. Rautenbach and Sereni [RS14] mentioned that $\operatorname{lpt}(G) \leq k+1$ for any such $G$. This result is a direct corollary of a theorem of Seymour and Thomas. (See Theorem 2.15 and Corollary 2.16). In fact, we need only the statement corresponding to the first part of the proof of Theorem 2.15. Next we state this as a previous lemma that is used in the proof of our main theorem.

Lemma 3.8. Let $G$ be a connected graph. Let $(T, \mathcal{V})$ be a tree decomposition of $G$. There exists a node $t \in V(T)$ such that $V_{t}$ is a longest path transversal.

Proof. Suppose by contradiction that this is not the case. For every $t \in V(T)$, let $\mathscr{P}(t)$ be the set of longest paths in $G$ that do not intersect $V_{t}$. Then, $\mathscr{P}(t) \neq \varnothing$ for every $t \in V(T)$. Thus, by Lemma 2.14, there exists an edge $t t^{\prime} \in E(T)$ and two paths $P \in \mathscr{P}(t)$ and $Q \in \mathscr{P}\left(t^{\prime}\right)$ such that $\operatorname{Branch}_{t}(P)=\operatorname{Branch}_{t}\left(t^{\prime}\right)$ and $\operatorname{Branch}_{t^{\prime}}(Q)=\operatorname{Branch}_{t^{\prime}}(t), P$ does not intersect $V_{t}$, and $Q$ does not intersect $V_{t^{\prime}}$. Therefore, $P$ and $Q$ do not intersect each other, a contradiction. 
The following proposition is useful for our main result.

Proposition 3.9. Let $G$ be a graph. Let $(T, \mathcal{V})$ be a tree decomposition of $G$. Let $t t^{\prime} \in E(T)$. Let $P^{\prime}$ be a path fenced by $V_{t}$ that 1-intersects $V_{t}$ such that $\operatorname{Branch}_{t}\left(P^{\prime}\right)=\operatorname{Branch}_{t}\left(t^{\prime}\right)$. Let $Q^{\prime}$ be a path fenced by $V_{t}$ that 1-intersects $V_{t}$ at a vertex in $V_{t} \backslash V_{t^{\prime}}$. Then $V\left(P^{\prime}\right) \cap V\left(Q^{\prime}\right)=\varnothing$.

Proof. Suppose by contradiction that $V\left(P^{\prime}\right) \cap V\left(Q^{\prime}\right) \neq \varnothing$. As $V\left(P^{\prime}\right) \cap V\left(Q^{\prime}\right) \cap V_{t}=\varnothing$, we must have that $\operatorname{Branch}_{t}\left(Q^{\prime}\right)=\operatorname{Branch}_{t}\left(P^{\prime}\right)=\operatorname{Branch}_{t}\left(t^{\prime}\right)$. By Proposition 2.8. $V_{t} \cap V\left(Q^{\prime}\right) \subseteq V_{t^{\prime}}$, a contradiction.

We can finally prove our main result. Recall that if $G$ is a graph with treewidth $k$, then $(T, \mathcal{V})$ is a full tree decomposition of $G$ if $\left|V_{t}\right|=k+1$ for every $t \in V(T)$, and $\left|V_{t} \cap V_{t^{\prime}}\right|=k$ for every $t t^{\prime} \in E(T)$ (Section 2.2). Recall also that $L=L(G)$ is the length of a longest path in $G$.

Theorem 3.10. For every connected partial $k$-tree $G$, we have $\operatorname{lpt}(G) \leq k$.

Proof. Let $(T, \mathcal{V})$ be a full tree decomposition of $G$, which exists by Proposition 2.1. By Lemma 3.8, there exists a node $t \in V(T)$ such that $V_{t}$, of cardinality $k+1$, is a longest path transversal. Suppose by contradiction that $\operatorname{lpt}(G)>k$. Then, no set of $k$ vertices in $V_{t}$ is a longest path transversal. As every longest path intersects $V_{t}$ at least once, for every vertex in $V_{t}$, there exists a longest path that 1-intersects $V_{t}$ at that vertex. Let $P$ be a longest path that intersects $V_{t}$ at $x$, let $P^{\prime}$ and $P^{\prime \prime}$ be the two $x$-tails of $P$. We show that

$$
\left|P^{\prime}\right|,\left|P^{\prime \prime}\right|>0 \text {, and } \operatorname{Branch}_{t}\left(P^{\prime}\right) \neq \operatorname{Branch}_{t}\left(P^{\prime \prime}\right) .
$$

Proof of (3.6): If $\left|P^{\prime}\right|=\left|P^{\prime \prime}\right|=0$, then $L=0$ and the proof follows trivially. Hence, we may assume without loss of generality that $\left|P^{\prime}\right|>0$. So, by Proposition 2.13 , there exists a node $t^{\prime} \in N(t)$ such that $\operatorname{Branch}_{t}\left(P^{\prime}\right)=\operatorname{Branch}_{t}\left(t^{\prime}\right)$. As $(T, \mathcal{V})$ is a full tree decomposition, there exists a vertex $y \in V_{t} \backslash V_{t^{\prime}}$. By Proposition 2.8, $x \in V_{t^{\prime}}$, so $y \neq x$. Let $Q$ be a longest path that 1-intersects $V_{t}$ at $y$. Let $Q^{\prime}$ and $Q^{\prime \prime}$ be the two $y$-tails of $Q$. By Proposition 3.9. both $V\left(P^{\prime}\right) \cap V\left(Q^{\prime}\right)$ and $V\left(P^{\prime}\right) \cap V\left(Q^{\prime \prime}\right)$ are empty. Hence, $V\left(P^{\prime \prime}\right) \cap V(Q) \neq \varnothing$, which implies that $\left|P^{\prime \prime}\right|>0$. Moreover, $\operatorname{Branch}_{t}\left(P^{\prime}\right) \neq \operatorname{Branch}_{t}\left(P^{\prime \prime}\right)$, otherwise, by Proposition 3.9, both $V\left(P^{\prime \prime}\right) \cap V\left(Q^{\prime}\right)$ and $V\left(P^{\prime \prime}\right) \cap V\left(Q^{\prime \prime}\right)$ are empty.

By (3.6), there exist two different nodes $t^{\prime}, t^{\prime \prime} \in N(t)$ such that $\operatorname{Branch}_{t}\left(P^{\prime}\right)=\operatorname{Branch}_{t}\left(t^{\prime}\right)$ and $\operatorname{Branch}_{t}\left(P^{\prime \prime}\right)=\operatorname{Branch}_{t}\left(t^{\prime \prime}\right)$. As $(T, \mathcal{V})$ is a full tree decomposition, there exists a vertex $a \in V_{t} \backslash V_{t^{\prime}}$ and a vertex $b \in V_{t} \backslash V_{t^{\prime \prime}}$. As $t^{\prime} \neq t^{\prime \prime}$, we have that $V_{t^{\prime}} \neq V_{t^{\prime \prime}}$ and $a \neq b$. Let $Q$ and $R$ be corresponding longest paths that 1-intersect $V_{t}$ at $a$ and $b$ respectively. By (3.6) (with either $Q$ or $R$ instead of $P$ ), both $Q$ and $R$ cross $V_{t}$. Observe that $x \in V_{t^{\prime}} \cap V_{t^{\prime \prime}}$ by Proposition 2.8, hence $a \neq x$ and $b \neq x$. 
Let $Q^{\prime}$ and $Q^{\prime \prime}$ be the two $a$-tails of $Q$, and let $R^{\prime}$ and $R^{\prime \prime}$ be the two $b$-tails of $R$. By Proposition 3.9. $V\left(P^{\prime}\right) \cap V(Q)=\varnothing$. So, $V\left(P^{\prime \prime}\right) \cap V(Q) \neq \varnothing$. We may assume, without loss of generality, that $Q^{\prime \prime}$ intersects $P^{\prime \prime}$, thus $\operatorname{Branch}_{t}\left(Q^{\prime \prime}\right)=\operatorname{Branch}_{t}\left(P^{\prime \prime}\right)=\operatorname{Branch}_{t}\left(t^{\prime \prime}\right)$. Analogously, with a similar analysis with $R$ instead of $Q$, we may assume that $\operatorname{Branch}_{t}\left(R^{\prime}\right)=\operatorname{Branch}_{t}\left(P^{\prime}\right)=\operatorname{Branch}_{t}\left(t^{\prime}\right)$. Applying (3.6) with $Q$ and $R$ instead of $P$, one can show that $\operatorname{Branch}_{t}\left(Q^{\prime}\right) \neq \operatorname{Branch}_{t}\left(Q^{\prime \prime}\right)$ and that $\operatorname{Branch}_{t}\left(R^{\prime}\right) \neq \operatorname{Branch}_{t}\left(R^{\prime \prime}\right)$. Thus, $Q^{\prime}$ is disjoint from $P$, and $R^{\prime \prime}$ is disjoint from $P$. Also, as $\operatorname{Branch}_{t}\left(R^{\prime}\right)=\operatorname{Branch}_{t}\left(t^{\prime}\right)$, by Proposition 3.9 paths $Q$ and $R^{\prime}$ do not intersect. Analogously, $R$ and $Q^{\prime \prime}$ do not intersect (Figure $3.4(\mathrm{a})$ ).

Let $a^{\prime} \in V\left(P^{\prime \prime}\right) \cap V\left(Q^{\prime \prime}\right)$ be such that the subpath of $P$ with extremes $x$ and $a^{\prime}$ is internally disjoint from $Q^{\prime \prime}$. Let $Q_{1}$ and $Q_{2}$ be the two $a^{\prime}$-tails of $Q$, with $Q_{1}$ containing $a$. Let $b^{\prime} \in V\left(P^{\prime}\right) \cap V\left(R^{\prime}\right)$ be such that the subpath of $P$ with extremes $x$ and $b^{\prime}$ is internally disjoint from $R^{\prime}$. Let $R_{1}$ and $R_{2}$ be the two $b^{\prime}$-tails of $R$, with $R_{1}$ containing $b$. Let $\tilde{P}$ be the subpath of $P$ that has $a^{\prime}$ and $b^{\prime}$ as extremes. As $\tilde{P}$ is internally disjoint from both $Q$ and $R$, we have that $R_{1} \cdot \tilde{P} \cdot Q_{2}$ and $Q_{1} \cdot \tilde{P} \cdot R_{2}$ are paths (Figure 3.4(b)), whose lengths sum more than $2 L$, a contradiction.

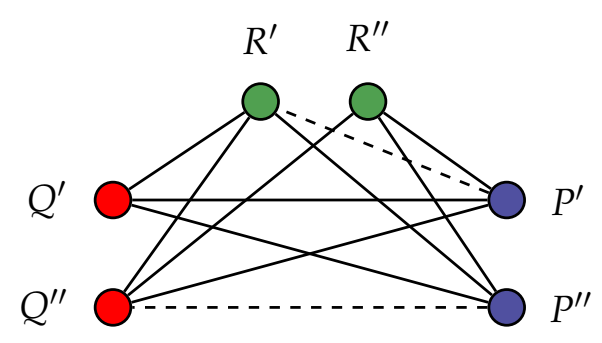

(a)

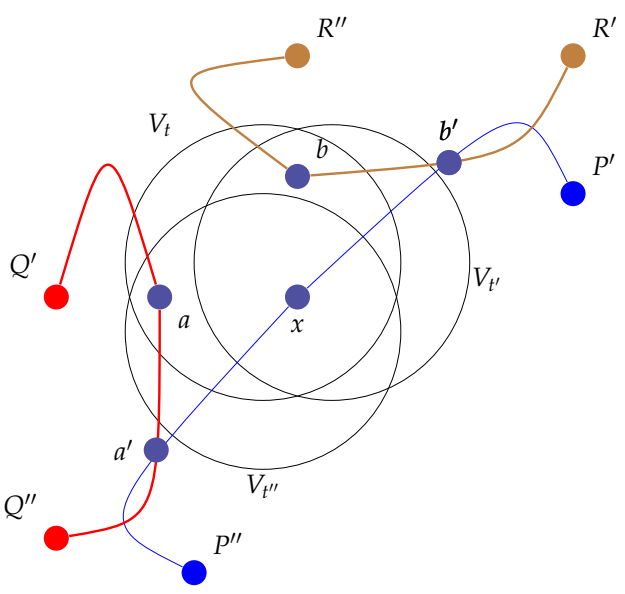

(b)

Figure 3.4: (a) Paths $P, Q$ and $R$ from the proof of Theorem 3.10 and a tripartite graph representing the interaction between their parts. The vertex set of the graph has two vertices for each of the paths, one for each part. The edge set contains two types of edges: the straight edges connect parts that are in different components of $G-V_{t}$ and the dashed edges connect parts that are in the same component of $G-V_{t}$. When the interaction between two parts is not determined, we omit the edge between them. (b) Vertices $a^{\prime}$ and $b^{\prime}$ used to obtain the final contradiction.

Planar graphs do not have bounded treewidth, because a grid graph on $n$ vertices has treewidth $\sqrt{n}$. However, Fomin and Thilikos [FT06] showed that any planar graph $G$ on $n$ vertices has treewidth at most $3.182 \sqrt{n}$. More generally, Alon, Seymour, and 
Thomas [AST90] showed that any $K_{r}$-minor free graph on $n$ vertices has treewidth at most $r^{1.5} \sqrt{n}$. Hence, we have the following corollary for Theorem 3.10. It improves the upper bound of $9 \sqrt{n} \lg n$ given by Rautenbach and Sereni when the graph is planar [RS14].

Corollary 3.11. For every connected planar graph $G$ on $n$ vertices, we have $\operatorname{lpt}(G) \leq 3.182 \sqrt{n}$, and for every connected $K_{r}$-minor free graph $G$, we have $\operatorname{lpt}(G) \leq r^{1.5} \sqrt{n}$.

\subsection{Full Substar Graphs}

In this section, we prove that all longest paths intersect in connected full substar graphs (Theorem 3.14). A graph $G$ is a full substar graph if there exists a tree $T$ and a collection $\mathscr{S}=\left\{S_{v}: v \in V(G)\right\}$ of stars in $T$ such that: $(i)\left|V\left(S_{v}\right)\right| \geq d_{T}\left(x_{v}\right)$ for every $v \in V(G)$, where $x_{v}$ is the center of $S_{v}$; and $(i i) V\left(S_{u}\right) \cap V\left(S_{v}\right) \neq \varnothing$ for every $u v \in E(G)$. We call the pair $(T, \mathscr{S})$ the intersection model of $G$. To avoid confusion, we call the vertices of $T$ as nodes. An example of a full substar graph can be seen in Figure 3.5 .
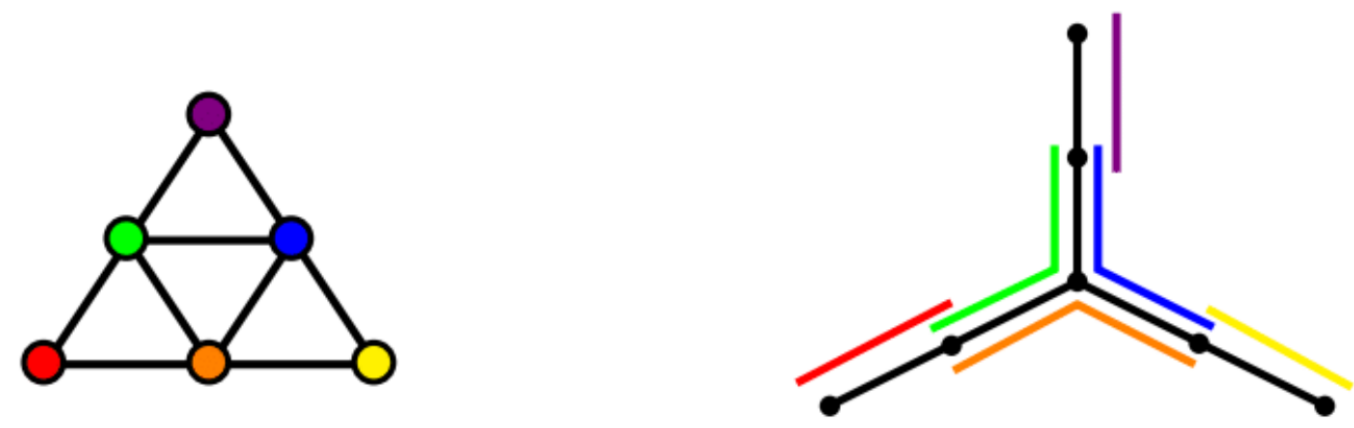

Figure 3.5: A full substar graph and a corresponding intersection model for the graph.

The definition of branch by Heinz [Hei13] (Section 2.4) applies naturally to an arbitrary tree. Here we applied it to the tree $T$. For a node $t \in V(T)$, let $\mathcal{C}_{t}$ be the set of vertices of $G$ whose corresponding stars have $t$ as center and $\mathcal{D}\left(t, t^{\prime}\right)$ be the set of vertices of $G$ whose stars are centered in a node that belongs to $\operatorname{Branch}_{t}\left(t^{\prime}\right)$.

In what follows, $G$ is a connected full substar graph and $(T, \mathscr{S})$ is an intersection model of $G$.

Lemma 3.12. Let $t \in V(T)$ and $x \in \mathcal{C}_{t}$. If $P$ is a longest path in $G$ such that $x \notin V(P)$, then there exists a node $t^{\prime} \in N_{T}(t)$ such that the following conditions hold:

(i) $V(P) \subseteq \mathcal{C}_{t} \cup \mathcal{D}\left(t, t^{\prime}\right)$ ，

(ii) $\left|V(P) \cap \mathcal{C}_{t}\right| \leq 1$ 
Moreover, if $\left|V(P) \cap \mathcal{C}_{t}\right|=1$, then $t^{\prime} \notin V\left(S_{x}\right)$.

Proof. First suppose that $V(P) \cap \mathcal{C}_{t}=\varnothing$. Suppose by contradiction that $(i)$ is false. Then $P$ has vertices whose substars are centered in two different branches of $T$ at $t$. Since $P$ contains no vertex of $\mathcal{C}_{t}$, then $P$ must contain two consecutive vertices whose stars are centered in $N_{T}(t)$. That is, $P$ contains two consecutive vertices that are adjacent to $x$, a contradiction. This implies that $(i)$ holds in this case and, since $\left|V(P) \cap \mathcal{C}_{t}\right|=0$, (ii) also holds.

Now assume that $V(P) \cap \mathcal{C}_{t} \neq \varnothing$. Suppose by a contradiction that $(i)$ does not hold. Then $P$ has vertices whose substars are centered in two different branches of $T$ at $t$. Let $u$ and $v$ be such vertices. Moreover, suppose that the subpath $\tilde{P}$ of $P$ with extremes $u$ and $v$ is minimum over all choices of $u$ and $v$. If $|\tilde{P}|=1$ then we are in a previous case. Hence, by the choice of $u$ and $v$, every internal vertex of $\tilde{P}$ is in $\mathcal{C}_{t}$. Since $\left|S_{x}\right| \geq d_{T}(t)$, one of $\{u, v\}$ is adjacent to $x$. Without loss of generality, let $u$ be such vertex and let $y$ be such that $u y \in E(\tilde{P})$. As $y \in \mathcal{C}_{t}$, we have that $y$ is also adjacent to $x$, a contradiction, because $P$ is a longest path. We conclude that $(i)$ holds in this case. Let $t^{\prime}$ be as stated in $(i)$.

For showing (ii), suppose by contradiction that $\left|V(P) \cap \mathcal{C}_{t}\right|>1$. Let $b, f \in V(P) \cap \mathcal{C}_{t}$. If $b f \in E(P)$, then we can add $x$ to $P$ between these two vertices, a contradiction to the fact that $P$ is a longest path. Observe also that $b$ is not an extreme of $P$. Hence, we may assume that $P=P_{a} \cdot a b \cdot b c \cdot P_{c d} \cdot d f \cdot P_{f}$, where $P_{a}$ and $P_{f}$ contain the extremes of $P$, and possibly $c=d,\left|P_{a}\right|=0$ or $\left|P_{f}\right|=0$. Note that $a, c, d \notin N(x)$, hence $t \notin S_{a}$, $t \notin S_{c}$, and $t \notin S_{d}$. This implies, as $a b, b c, d f \in E(G)$, that $S_{a} \cap S_{b}=\left\{t^{\prime}\right\}, S_{b} \cap S_{c}=\left\{t^{\prime}\right\}$ and $S_{d} \cap S_{f}=\left\{t^{\prime}\right\}$. So, $t^{\prime} \in S_{a} \cap S_{b} \cap S_{c} \cap S_{d} \cap S_{f}$ and $\{a, b, c, d, f\}$ induces a clique. But then, $P_{a} \cdot a c \cdot P_{c d} \cdot d b \cdot b x \cdot x f \cdot P_{f}$ is a path longer than $L$, a contradiction (Figure 3.6).

To finish the proof, suppose by contradiction that $\left|V(P) \cap \mathcal{C}_{t}\right|=1$ and $t^{\prime} \in S_{x}$. Let $\{a\}=V(P) \cap \mathcal{C}_{t}$. Since $|P| \geq 1$ and $V(P) \subseteq \mathcal{C}_{t} \cup \mathcal{D}\left(t, t^{\prime}\right)$, there exists an edge $a b \in E(P)$ such that $b \in \mathcal{D}\left(t, t^{\prime}\right)$. This implies that $t^{\prime} \in S_{b}$, so $x$ is adjacent to both $a$ and $b$, a contradiction.

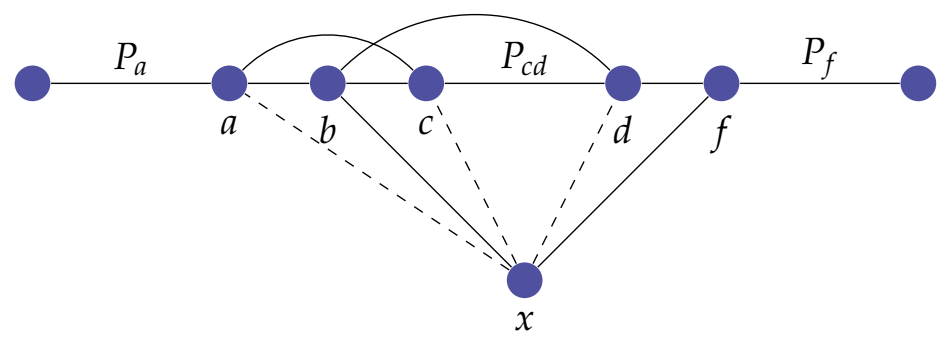

Figure 3.6: Situation in the proof of Lemma 3.12 
Lemma 3.13. Let $t \in V(T)$. If $\operatorname{lpt}(G)>1$, then there exists a longest path $P$ in $G$ and $a$ node $t^{\prime} \in N_{T}(t)$ such that $V(P) \subseteq \mathcal{D}\left(t, t^{\prime}\right)$.

Proof. Suppose for a moment that $\mathcal{C}_{t}=\varnothing$. If there is no vertex $v \in V(G)$ such that $t \in S_{v}$, then any longest path satisfies the condition and the proof follows. Thus, let $x \in V(G)$ such that $t \in S_{x} . \operatorname{As} \operatorname{lpt}(G)>1$, there exists a longest path $P$ that does not contain $x$. If there exists a node $t^{\prime} \in N_{T}(t)$ such that $V(P) \subseteq \mathcal{D}\left(t, t^{\prime}\right)$, we are done. So, as $\mathcal{C}_{t}=\varnothing$, there exists an edge $u v \in E(P)$ such that $u \in \mathscr{C}_{t_{1}}, v \in \mathscr{C}_{t_{2}}, t_{1} \neq t_{2}$, and $t_{1}, t_{2} \in N_{T}(t)$. As $t \in S_{u} \cap S_{v} \cap S_{x}$, we have that $x$ is adjacent to both $u$ and $v$, a contradiction. Thus, we may assume that $\mathcal{C}_{t} \neq \varnothing$.

Let $x \in \mathcal{C}_{t}$. Moreover, suppose that $\left|V\left(S_{x}\right)\right|$ is maximum over all such $x$. Since $\operatorname{lpt}(G)>1$, there exists a longest path $P$ in $G$ that does not contain $x$. By Lemma 3.12, there exists a node $t^{\prime} \in N_{T}(t)$ such that $V(P) \in \mathcal{C}_{t} \cup \mathcal{D}\left(t, t^{\prime}\right)$. If $V(P) \cap \mathcal{C}_{t}=\varnothing$, the statement holds. Otherwise, $P$ has a vertex $a$ such that $a \in \mathcal{C}_{t}$. Also, by Lemma 3.12, $V(P) \cap \mathcal{C}_{t}=\{a\}$. Moreover, by the last part of Lemma 3.12 . $t^{\prime} \notin S_{x}$. This implies that $\left|V\left(S_{x}\right)\right|=d_{T}(t)$. And, by the choice of $x,\left|V\left(S_{a}\right)\right|=d_{T}(t)$. That is, both $S_{x}$ and $S_{a}$ miss a node in the neighborhood of $t$. As $S_{x} \neq S_{a}$, there exists a node $t^{\prime \prime} \in S_{x} \backslash S_{a}$.

Since $\operatorname{lpt}(G)>1$, there exists a longest path $Q$ in $G$ that does not contain $a$. By Lemma 3.12, there exists a node $p \in N_{T}(t)$ such that $V(Q) \subseteq \mathcal{C}_{t} \cup \mathcal{D}(t, p)$. If $V(Q) \subseteq \mathcal{D}(t, p)$ we are done. Hence, $\left|V(Q) \cap \mathcal{C}_{t}\right|=1$ and, as $t^{\prime \prime} \notin S_{a}$, by the last part of Lemma 3.12, $p=t^{\prime \prime}$. Then $V(P) \subseteq\{a\} \cup \mathcal{D}\left(t, t^{\prime}\right)$ and $V(Q) \subseteq\{b\} \cup \mathcal{D}\left(t, t^{\prime \prime}\right)$. As $a \neq b$ and $\mathcal{D}\left(t, t^{\prime}\right) \cap \mathcal{D}\left(t, t^{\prime \prime}\right)=\varnothing, P$ and $Q$ do not intersect each other, a contradiction.

We are now ready to prove the main result of this section.

Theorem 3.14. If $G$ is a connected full substar graph, then $\operatorname{lpt}(G)=1$.

Proof. Suppose by a contradiction that $\operatorname{lpt}(G)>1$. Let $(T, \mathscr{S})$ be an intersection model of $G$. We define a partial orientation $T^{\prime}$ of $T$ as follows: $t t^{\prime} \in E\left(T^{\prime}\right)$ if and only if $t t^{\prime} \in E(T)$ and there exists a path $P$ of $G$ such that $V(P) \subseteq \mathcal{D}\left(t, t^{\prime}\right)$. Hence every node in $T^{\prime}$ has outdegree at least one by Lemma 3.13. Let $t t^{\prime}$ be the last arc of a maximal directed path in $T^{\prime}$. As $T$ is a tree, $t^{\prime} t$ is also an arc in $T^{\prime}$, which implies that there exist two longest paths $P$ and $Q$ in $G$ such that $V(P) \subseteq \mathcal{D}\left(t, t^{\prime}\right)$ and $V(Q) \subseteq \mathcal{D}\left(t^{\prime}, t\right)$. But then, as $\mathcal{D}\left(t, t^{\prime}\right) \cap \mathcal{D}\left(t^{\prime}, t\right)=\varnothing, P$ and $Q$ do not intersect each other, a contradiction.

\subsection{Bipartite Permutation Graphs}

In this section, we prove that all longest paths intersect in connected bipartite permutation graphs (Theorem 3.25). We begin by introducing the corresponding definition. 
Let $\mathcal{L}_{1}$ and $\mathcal{L}_{2}$ be two parallel lines in the plane. Consider two sets $X=\left\{x_{1}, x_{2}, \ldots, x_{n}\right\}$ and $Y=\left\{y_{1}, y_{2}, \ldots, y_{m}\right\}$ of segments that join points in $\mathcal{L}_{1}$ with points in $\mathcal{L}_{2}$, such that the extremes of every two elements in $X \cup Y$ are pairwise disjoint. Moreover, every two elements in $X$ do not intersect each other and every two elements in $Y$ do not intersect each other.

Let $\sigma$ be the function that maps the extreme in $\mathcal{L}_{1}$ of a segment to the other extreme. That is, if $r_{i}$ is the extreme in $\mathcal{L}_{1}$ of a segment $x_{i}$ in $X$, then the other extreme is $\sigma\left(r_{i}\right)$; and if $s_{i}$ is the extreme in $\mathcal{L}_{1}$ of a segment $y_{i}$ in $Y$, then the other extreme is $\sigma\left(s_{i}\right)$. Consider an associated bipartite graph $G=(X, Y, E)$ where $x y \in E$ if and only if the segments $x$ and $y$ intersect each other. We call the tuple $\left(\mathcal{L}_{1}, \mathcal{L}_{2}, X \cup Y, \sigma\right)$ a line representation of $G$. A graph is called a bipartite permutation graph if it has a line representation.

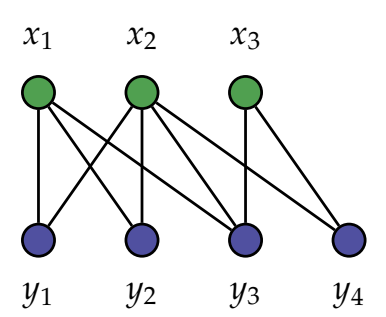

(a)

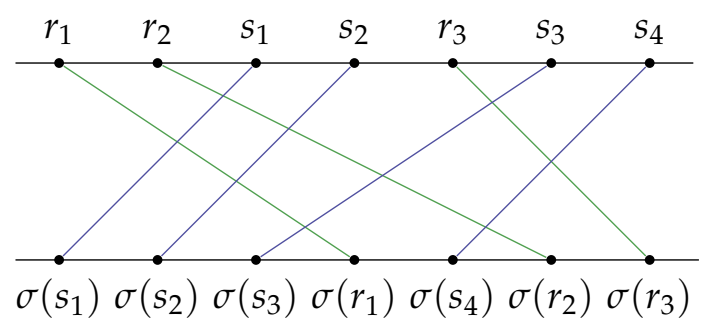

(b)

Figure 3.7: (a) A bipartite permutation graph. (b) A corresponding line representation.

In what follows, we assume that $G=(X, Y, E)$ is a connected bipartite permutation graph, with a line representation $\left(\mathcal{L}_{1}, \mathcal{L}_{2}, X \cup Y, \sigma\right)$, where $X=\left\{x_{1}, x_{2}, \ldots, x_{n}\right\}$ and $Y=$ $\left\{y_{1}, y_{2}, \ldots, y_{m}\right\}$. We also assume that $r_{i}$ is the extreme of $x_{i}$ in $\mathcal{L}_{1}$ and that $s_{i}$ is the extreme of $y_{i}$ in $\mathcal{L}_{1}$. Moreover, we consider that the sets $X$ and $Y$ are ordered by their extremes in $\mathcal{L}_{1}$. That is, $r_{i}<r_{j}$ if and only if $i<j$, for every $x_{i}$ and $x_{j}$ in $X$; and $s_{i}<s_{j}$ if and only if $i<j$, for every $y_{i}$ and $y_{j}$ in $Y$ (Figure 3.7). For two elements $x_{i}$ and $x_{j}$ in $X$ with $i<j$, we also say that $x_{i}<x_{j}$, and we do the same for $Y$. Next we show some basic properties of bipartite permutation graphs.

Proposition 3.15. $\sigma\left(r_{1}\right)<\sigma\left(r_{2}\right)<\cdots<\sigma\left(r_{n}\right)$ and $\sigma\left(s_{1}\right)<\sigma\left(s_{2}\right)<\cdots<\sigma\left(s_{m}\right)$.

Proof. Suppose by contradiction that there exist $i$ and $j$ such that $r_{i}<r_{j}$ and $\sigma\left(r_{i}\right)>\sigma\left(r_{j}\right)$. Hence $x_{i}$ and $x_{j}$ would be adjacent, a contradiction to the fact that $G$ is bipartite. A similar proof applies for $s_{i}$ and $s_{j}$.

The next proposition says that the neighborhood of a vertex is either completely to its left or completely to its right.

Proposition 3.16. If $x_{i}, x_{j} \in N\left(y_{k}\right)$ for some $i, j, k$, then $\left(r_{i}-s_{k}\right)\left(r_{j}-s_{k}\right)>0$. In a similar way, if $y_{i}, y_{j} \in N\left(x_{k}\right)$ for some $i, j, k$, then $\left(s_{i}-r_{k}\right)\left(s_{j}-r_{k}\right)>0$. 
Proof. The two statements are symmetric, so we only analyze the first one. Suppose by contradiction that there exist $i, j, k$ such that $r_{i}<s_{k}<r_{j}$ and $r_{i}, r_{j} \in N\left(s_{k}\right)$. Then $\sigma\left(r_{j}\right)<$ $\sigma\left(s_{k}\right)<\sigma\left(r_{i}\right)$, a contradiction to Proposition 3.15 .

Proposition 3.17. If $x_{i}$ is adjacent to $y_{j_{1}}$ and $y_{j_{2}}$, with $j_{1} \leq j_{2}$, then $x_{i}$ is adjacent to every $y_{j}$ with $j_{1} \leq j \leq j_{2}$. If $x_{i_{1}}$ and $x_{i_{2}}$, with $i_{1} \leq i_{2}$, are adjacent to $y_{j}$, then $y_{j}$ is adjacent to every $x_{i}$ with $i_{1} \leq i \leq i_{2}$.

Proof. The two statements are symmetric, so we only analyze the first one. The case in which $j \in\left\{j_{1}, j_{2}\right\}$ is immediate, so we may assume that $j_{1}<j<j_{2}$. By Proposition 3.16, either $x_{i}<y_{j_{1}}<y_{j}<y_{j_{2}}$ or $y_{j_{1}}<y_{j}<y_{j_{2}}<x_{i}$. Consider the first case. As $x_{i} y_{j_{2}} \in E$, we have that $\sigma\left(s_{j_{2}}\right)<\sigma\left(r_{i}\right)$. Also, $\sigma\left(s_{j}\right)<\sigma\left(s_{j_{2}}\right)$ by Proposition 3.15. So $\sigma\left(s_{j}\right)<\sigma\left(r_{i}\right)$, implying that $x_{i}$ and $y_{j}$ are adjacent.

The next two properties are very important, as they are used repeatedly throughout the next proofs.

Proposition 3.18. If $x_{i_{1}} y_{j_{2}}, x_{i_{2}} y_{j_{1}} \in E$, with $i_{1} \leq i_{2}$ and $j_{1} \leq j_{2}$, then $x_{i_{1}} y_{j_{1}}, x_{i_{2}} y_{j_{2}} \in E$. In other words, $\left\{x_{i_{1}}, x_{i_{2}}, y_{j_{1}}, y_{j_{2}}\right\}$ induces a complete bipartite graph.

Proof. The case in which $j_{1}=j_{2}$ or $i_{1}=i_{2}$ is clear, so let us assume that $i_{1}<i_{2}$ and $j_{1}<j_{2}$. First suppose that $r_{i_{1}}<s_{j_{2}}$. Then $\sigma\left(s_{j_{2}}\right)<\sigma\left(r_{i_{1}}\right)$ because $x_{i_{1}} y_{j_{2}} \in E$. By Proposition 3.15, we have that $\sigma\left(r_{i_{1}}\right)<\sigma\left(r_{i_{2}}\right)$ and $\sigma\left(s_{j_{1}}\right)<\sigma\left(s_{j_{2}}\right)$. So

$$
\sigma\left(s_{j_{1}}\right)<\sigma\left(s_{j_{2}}\right)<\sigma\left(r_{i_{1}}\right)<\sigma\left(r_{i_{2}}\right)
$$

Hence, as $x_{i_{2}} y_{j_{1}} \in E$, we have that $r_{i_{2}}<s_{j_{1}}$, and

$$
r_{i_{1}}<r_{i_{2}}<s_{j_{1}}<s_{j_{2}}
$$

By (3.7) and (3.8), we derive that $x_{i_{1}} y_{j_{1}}, x_{i_{2}} y_{j_{2}} \in E$ (Figure 3.8(a)).

Now suppose that $r_{i_{1}}>s_{j_{2}}$. Then

$$
s_{j_{1}}<s_{j_{2}}<r_{i_{1}}<r_{i_{2}}
$$

As $x_{i_{2}} y_{j_{1}} \in E$, we have that $\sigma\left(r_{i_{2}}\right)<\sigma\left(s_{j_{1}}\right)$. Using Proposition 3.15, we deduce that

$$
\sigma\left(r_{i_{1}}\right)<\sigma\left(r_{i_{2}}\right)<\sigma\left(s_{j_{1}}\right)<\sigma\left(s_{j_{2}}\right)
$$

By (3.9) and (3.10), we derive that $x_{i_{1}} y_{j_{1}}, x_{i_{2}} y_{j_{2}} \in E$ (Figure $3.8(\mathrm{~b})$ ). 


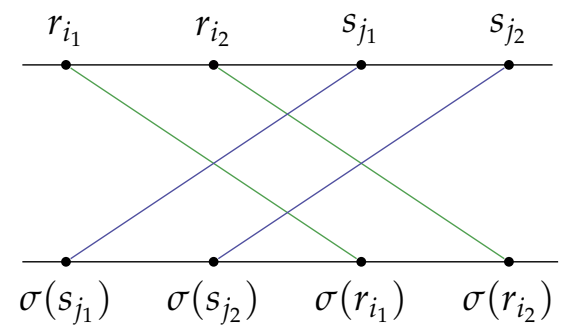

(a)

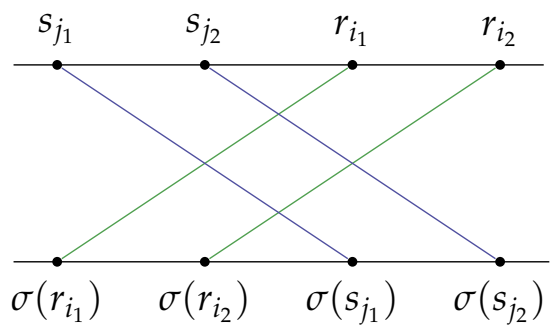

(b)

Figure 3.8: The two cases in the proof of Proposition 3.18

Proposition 3.19. Let $x_{i_{1}} \leq x_{i_{2}} \leq x_{i_{3}} \leq x_{i_{4}}$ be vertices in $X$ and $y_{j_{1}} \leq y_{j_{2}} \leq y_{j_{3}} \leq y_{j_{4}}$ be vertices in $Y$. If $x_{i_{1}} y_{j_{4}}$ and $x_{i_{4}} y_{j_{1}}$ are in $E$, then $x_{i_{2}} y_{j_{3}}, y_{j_{2}} x_{i_{3}}, x_{i_{2}} y_{j_{2}}$, and $x_{i_{3}} y_{j_{3}}$ are in $E$. In other words, $\left\{x_{i_{2}}, x_{i_{3}}, y_{j_{2}}, y_{j_{3}}\right\}$ induces a complete bipartite graph.

Proof. By Proposition 3.18, we have that $x_{i_{1}} y_{j_{1}}$ and $x_{i_{4}} y_{j_{4}}$ are in E. By applying Proposition 3.17, once for $x_{i_{1}}$ and once for $x_{i_{4}}$, we deduce that $x_{i_{1}} y_{j_{2}}, x_{i_{1}} y_{j_{3}}, x_{i_{4}} y_{j_{2}}, x_{i_{4}} y_{j_{3}}$ are in $E$, and now applying it for $y_{j_{2}}$ and for $y_{j_{3}}$, we deduce that $x_{i_{2}} y_{j_{2}}, x_{i_{3}} y_{j_{2}}, x_{i_{2}} y_{j_{3}}$ and $x_{i_{3}} y_{j_{3}}$ are in $E$.

Until now, we have used the line representation of $G$ to prove some properties. From now on, we will not need this line representation anymore. That is, we only need to concentrate in graph $G$, viewed as a bipartite graph that has the previous properties.

We are interested in the behaviour of longest paths in a bipartite permutation graph. We begin by showing that every longest path can be converted into another longest path with the same set of vertices that is ordered in a special way. As we only care about vertex intersection of longest paths, we are only interested in such ordered paths. To be more precise, if $P=a_{1} b_{1} a_{2} b_{2} \cdots a_{k} b_{k}$ is a path in $G$, we say that $P$ is ordered if $a_{1}<a_{2}<\cdots<a_{k}$ and $b_{1}<b_{2}<\cdots<b_{k}$. A similar definition applies when $P$ has even length.

Let $P$ be a path in $G$ with $P \cap X=\left\{a_{1}, a_{2}, \ldots, a_{|P \cap X|}\right\}$ and $P \cap Y=\left\{b_{1}, b_{2}, \ldots, b_{|P \cap Y|}\right\}$, so that $a_{1}<a_{2}<\cdots<a_{|P \cap X|}$ and $b_{1}<b_{2}<\cdots<b_{|P \cap Y|}$. For every $i \in\{1, \ldots,|P \cap X|\}$, let $X_{i}=\left\{a_{1}, a_{2}, \ldots, a_{i}\right\}$ and $\bar{X}_{i}=P \cap\left(X \backslash X_{i}\right)$. For every $i \in\{0, \ldots,|P \cap Y|\}$, let $Y_{i}=\left\{b_{1}, b_{2}, \ldots, b_{i}\right\}$ and $\bar{Y}_{i}=P \cap\left(Y \backslash Y_{i}\right)$. It is denoted by $d_{P}\left(X_{i}\right)$ the sum $\sum_{v \in X_{i}} d_{P}(v)$ and by $d_{P}\left(Y_{j}\right)$ the sum $\sum_{w \in Y_{j}} d_{P}(w)$.

Proposition 3.20. Let $i, j$ be such that $1 \leq i \leq|P \cap X|, 0 \leq j \leq|P \cap Y|$, and either $i<|P \cap X|$ or $j<|P \cap Y|$. Then, there exists either an edge in $P$ from $X_{i}$ to $\bar{Y}_{j}$, or an edge in $P$ from $Y_{j}$ to $\bar{X}_{i}$.

Proof. Without loss of generality, assume that $i<|P \cap X|$. Let $v \in P \cap\left(X \backslash X_{i}\right)$. Suppose 
by contradiction that no such edge exists. Then, there is no path, in the subgraph of $G$ induced by $E(P)$, between $v$ and a vertex in $X_{i} \cup Y_{j}$, a contradiction.

Proposition 3.21. Let $i, j$ be such that $1 \leq i \leq|P \cap X|, 0 \leq j \leq|P \cap Y|$, and either $i<|P \cap X|$ or $j<|P \cap Y|$. If $d_{P}\left(X_{i}\right) \geq d_{P}\left(Y_{j}\right)$, then there exists an edge from $X_{i}$ to $\bar{Y}_{j}$. If $d_{P}\left(Y_{j}\right) \geq d_{P}\left(X_{i}\right)$, then there exists an edge from $Y_{j}$ to $\bar{X}_{i}$.

Proof. We prove only the first affirmation, as the proof for the second one is analogous. Suppose by contradiction that $d_{P}\left(X_{i}\right) \geq d_{P}\left(Y_{j}\right)$ and there exists no edge from $X_{i}$ to $\bar{Y}_{j}$. By Proposition 3.20, there exists at least one edge from $Y_{j}$ to $\bar{X}_{i}$, so

$$
\begin{aligned}
d_{P}\left(Y_{j}\right) & =\left|\left\{w v \in E(P): w \in Y_{j}, v \in X_{i}\right\}\right|+\left|\left\{w v \in E(P): w \in Y_{j}, v \in \bar{X}_{i}\right\}\right| \\
& =\left|\left\{v w \in E(P): v \in X_{i}, w \in V(P)\right\}\right|+\left|\left\{w v \in E(P): w \in Y_{j}, v \in \bar{X}_{i}\right\}\right| \\
& =d_{P}\left(X_{i}\right)+\left|\left\{w v \in E(P): w \in Y_{j}, v \in \bar{X}_{i}\right\}\right| \\
& >d_{P}\left(X_{i}\right)
\end{aligned}
$$

a contradiction.

Lemma 3.22. For every path $P$ in a connected bipartite permutation graph $G=(X, Y, E)$, there exists an ordered path with the same vertex set as $P$.

Proof. Suppose that $P \cap X=\left\{a_{1}, a_{2}, \ldots, a_{|P \cap X|}\right\}, P \cap Y=\left\{b_{1}, b_{2}, \ldots, b_{|P \cap Y|}\right\}, a_{1}<\cdots<$ $a_{|P \cap X|}$ and $b_{1}<\cdots<b_{|P \cap Y|}$. Without loss of generality, we may assume that $|P \cap X| \geq$ $|P \cap Y|$ and that, if $|P \cap X|=|P \cap Y|$, then $i^{*} \leq j^{*}$, where $a_{i^{*}}$ is the extreme of $P$ in $X$ and $b_{j^{*}}$ is the extreme of $P$ in $Y$. (If $i^{*}>j^{*}$, then a similar proof applies by interchanging $X$ and $Y$.) Let $k=|P \cap Y|$. We show that

for every $i \in\{1, \ldots, k\}$, there exists an edge with one end in $X_{i}$ and the other in $\bar{Y}_{i-1}$,

and

$$
\text { for every } i \in\{1, \ldots, k-1+|P \cap X|-|P \cap Y|\} \text {, }
$$

there exists an edge with one end in $Y_{i}$ and the other in $\bar{X}_{i}$.

Proof of 3.11: Observe that $d_{P}(u)=1$ for at most two vertices $u$ in $X_{i}$ (the extremes of $P$ ). Therefore, $d_{P}\left(X_{i}\right) \geq 2\left|X_{i}\right|-2=2\left|Y_{i-1}\right|$. As $d_{P}(w) \leq 2$ for every $w \in Y_{i-1}$, we have that $d_{P}\left(Y_{i-1}\right) \leq 2\left|Y_{i-1}\right|$. Hence, $d_{P}\left(X_{i}\right) \geq d_{P}\left(Y_{i-1}\right)$ and, as $i-1<k$, 3.11 holds by Proposition 3.21 .

Proof of 3.12): First suppose that $|P \cap X|=k(=|P \cap Y|)$. As $i^{*} \leq j^{*}$, we have that $d_{P}\left(Y_{i}\right) \geq d_{P}\left(X_{i}\right)$. Indeed, if $a_{i^{*}} \in X_{i}$ then $d_{P}\left(X_{i}\right)=2\left|X_{i}\right|-2$ and $d_{P}\left(Y_{i}\right) \geq 2\left|Y_{i}\right|-2$, and if $a_{i^{*}} \notin X_{i}$ then, as $i^{*} \leq j^{*}, y_{j^{*}} \notin Y_{i}$, so $d_{P}\left(X_{i}\right)=2\left|X_{i}\right|$ and $d_{P}\left(Y_{i}\right)=2\left|Y_{i}\right|$. Thus, as $i<k,(3.12$ ) holds by Proposition 3.21. Now suppose that $|P \cap X|=k+1$. Then, 
$d_{P}(w)=2$ for every $w \in Y_{i}$. Therefore, $d_{P}\left(Y_{i}\right)=2\left|Y_{i}\right|=2\left|X_{i}\right|$. As $d_{P}(v) \leq 2$ for every $v \in X_{i}$, we have that $d_{P}\left(X_{i}\right) \leq 2\left|X_{i}\right|$. Hence, $d_{P}\left(Y_{i}\right) \geq d_{P}\left(X_{i}\right)$ and, as $i<k+1,3.12$ holds by Proposition 3.21 .

Let $i \in\{1, \ldots, k-1\}$. By (3.11), there exists a vertex $a_{q}$ in $X_{i}$ with a neighbor $b_{r^{\prime}}$ in $\bar{Y}_{i-1}$. By (3.12, there exists a vertex $b_{r}$ in $Y_{i}$ with a neighbor $a_{q^{\prime}}$ in $\bar{X}_{i}$. As $a_{q} \leq a_{i} \leq$ $a_{i+1} \leq a_{q^{\prime}}$ and $b_{r} \leq b_{i} \leq b_{i} \leq b_{r^{\prime}}$, by Proposition 3.19, both $a_{i} b_{i}$ and $b_{i} a_{i+1}$ are edges (Figure 3.9). By (3.11), $a_{k} b_{k}$ is an edge, hence $a_{1} b_{1} \cdots a_{k} b_{k}$ is a path. This implies that if $|X \cap P|=k$, we are done. Also, if $|X \cap P|=k+1$, then $b_{k} a_{k+1}$ is an edge, by (3.12), so $a_{1} b_{1} \cdots a_{k} b_{k} a_{k+1}$ is a path.

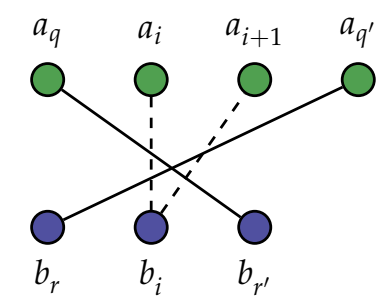

Figure 3.9: Situation in the proof of Lemma 3.22

As observed before, Lemma 3.22 says that we can restrict attention to ordered longest paths from now on. Remember that we want to prove that $\operatorname{lpt}(G)=1$. We proceed in two steps. First, we prove that $\operatorname{lpt}(G) \leq 2$. In fact, we prove that the ends of every edge form a longest path transversal. Finally, we prove that one element in $\left\{x_{1}, y_{1}\right\}$ is also a longest path transversal, which implies that $\operatorname{lpt}(G)=1$.

Let $x_{i_{1}} y_{j_{1}}$ and $x_{i_{2}} y_{j_{2}}$ be two edges in $G$. We say that $x_{i_{1}} y_{j_{1}}$ hits $x_{i_{2}} y_{j_{2}}$ if $\left(i_{1}-i_{2}\right)\left(j_{1}-j_{2}\right)<0$. If this is not the case, we say they are parallel. We also say that $\left|i_{1}-i_{2}\right|$ is the distance in $X$ and that $\left|j_{1}-j_{2}\right|$ is the distance in $Y$ between such edges. It is denoted by $\operatorname{dist}_{X}\left(x_{i_{1}} y_{j_{1}}, x_{i_{2}} y_{j_{2}}\right)$ and $\operatorname{dist}_{Y}\left(x_{i_{1}} y_{j_{1}}, x_{i_{2}} y_{j_{2}}\right)$ these two values, respectively.

Proposition 3.23. Let $P$ be a longest path and $x_{i_{1}} y_{j_{1}} \in E(P)$. Let $v w \in E(G)$. If $x_{i_{1}} y_{j_{1}}$ hits $v w$, then P contains at least one of $\{v, w\}$.

Proof. Suppose by contradiction that $\{v, w\} \cap V(P)=\varnothing$. Without loss of generality, suppose that $x_{i_{1}}<v$ and $y_{j_{1}}>w$. By Proposition 3.18, $x_{i_{1}} w$ and $v y_{j_{1}}$ are edges. Therefore, $\left(P-x_{i_{1}} y_{j_{1}}\right) \cdot x_{i_{1}} w v y_{j_{1}}$ is a path longer than $P$, a contradiction.

Lemma 3.24. Let $G=(X, Y, E)$ be a connected bipartite permutation graph. Let vw $\in E$, with $v \in X$ and $w \in Y$. Every ordered longest path contains a vertex of $\{v, w\}$.

Proof. Suppose by contradiction that there exists an ordered longest path $P$ that does not contain either $v$ or $w$. Then, by Proposition 3.23, all edges of $P$ are parallel to $v w$. Let $x_{i_{1}} y_{j_{1}}$ be the edge of $P$ that is "closer" to $v w$. That is, dist $t_{X}\left(x_{i_{1}} y_{j_{1}}, v w\right)=$ 
$\min \left\{\operatorname{dist}_{X}(e, v w): e \in E(P)\right\}$ and $\operatorname{dist}_{Y}\left(x_{i_{1}} y_{j_{1}}, v w\right)=\min \left\{\operatorname{dist}_{Y}(e, v w): e \in E(P)\right\} . \mathrm{Ob}-$ serve that, as $P$ is an ordered path, one of $\left\{x_{i_{1}}, y_{j_{1}}\right\}$ is an extreme of $P$. Suppose that $x_{i_{1}}$ is such an extreme. (A similar proof applies when this is not the case.) Without loss of generality, we may assume that $x_{i_{1}}>v$ and that $P$ is a path with minimum value of $x_{i_{1}}$ among all such paths.

Let $H$ be the subgraph of $G$ induced by the vertices $\left\{x_{i}: i \geq i_{1}\right\} \cup\left\{y_{j}: j \geq j_{1}\right\}$. As $G$ is connected and $G \neq H$, there exists an edge between $H$ and $G-V(H)$. First suppose that such an edge is between a vertex $x_{\ell}$ in $H$ and a vertex $y_{r}$ in $G-V(H)$. Then, by Proposition 3.18, $x_{i_{1}}$ is adjacent to $y_{r}$. Hence, $y_{r} x_{i_{1}} \cdot P$ is a path longer than $P$, a contradiction (Figure 3.10(a)). Now, suppose that there is an edge between a vertex $x_{\ell}$ in $G-V(H)$ and a vertex $y_{r}$ in $H$. Then, by Proposition 3.18, $x_{l}$ is adjacent to $y_{j_{1}}$. So $Q=\left(P-x_{i_{1}} y_{j_{1}}\right) \cdot x_{\ell} y_{j_{1}}$ is also a longest path. As $V(Q) \backslash V(P)=\left\{x_{\ell}\right\}$, we have that $w \notin Q$. Observe also that $v \notin Q$, otherwise $Q \cdot v w$ is a path longer than $P$. Hence, by Proposition 3.23, all edges of $Q$ are parallel to $v w$, which implies that $v<x_{\ell}<x_{i_{1}}$, a contradiction to the way $P$ was chosen (Figure $3.10(\mathrm{~b})$ ).

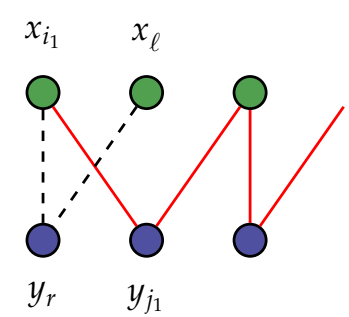

(a)

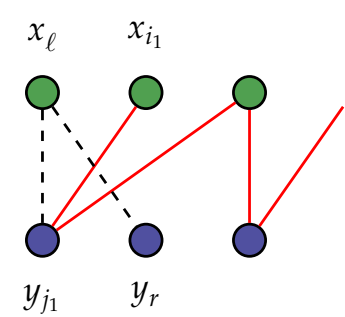

(b)

Figure 3.10: The two cases in the proof of Lemma 3.24 The solid lines represent the edges in $P$.

Given a collection $\mathcal{C}$ of ordered longest paths, we say that $P \in \mathcal{C}$ is a left-most path if, for every other path $Q \in \mathcal{C}$ and for every $i$, the $i$-th vertex of $P$ in $X$ is less than or equal to the $i$-th vertex of $Q$ in $X$, and the same applies for $Y$ instead of $X$. Such a path exists because all paths in $\mathcal{C}$ are ordered. Recall that $X=\left\{x_{1}, x_{2}, \ldots, x_{n}\right\}$ and $Y=\left\{y_{1}, y_{2}, \ldots, y_{m}\right\}$.

Theorem 3.25. For every connected bipartite permutation graph $G=(X, Y, E), \operatorname{lpt}(G)=1$.

Proof. Suppose by contradiction that $\operatorname{lpt}(G)>1$. Then, there exists a longest path $P$ that does not contain $y_{1}$ and a longest path $Q$ that does not contain $x_{1}$. We may assume, by Lemma 3.22 , that $P$ and $Q$ are ordered. As $G$ is connected, we have $d\left(x_{1}\right), d\left(y_{1}\right)>0$, so $x_{1} y_{1}$ is an edge by Proposition 3.18. Hence, by Lemma 3.24, $x_{1} \in P$ and $y_{1} \in Q$. We may assume that both $P$ and $Q$ are left-most paths. Suppose without loss of generality that $n \geq m$. Thus, for all $i \in\{2, \ldots, m\}$, it suffices to prove the following conditions: 
(a) $y_{i}$ is the $(2 i-3)$-th vertex of $P, x_{i-1}$ is the $(2 i-2)$-th vertex of $P, x_{i}$ is the $(2 i-3)$-th vertex of $Q$, and $y_{i-1}$ is the $(2 i-2)$-th vertex of $Q$.

(b) $x_{i} y_{i}$ is an edge.

Indeed, if that is the case, then we would have a path $R=x_{1} y_{1} x_{2} y_{2} \cdots x_{m} y_{m}$ of length $2 m-1$. As $P$ does not contain $y_{1}$, we would have $|P \cap Y|=\mid P \cap$ $\left\{y_{2}, y_{3}, \ldots, y_{m}\right\} \mid=m-1$. And, as $G$ is bipartite, $|P \cap X| \leq m$. Hence $|P| \leq 2 m-2<|R|$, a contradiction, because $P$ is a longest path (Figure 3.11.

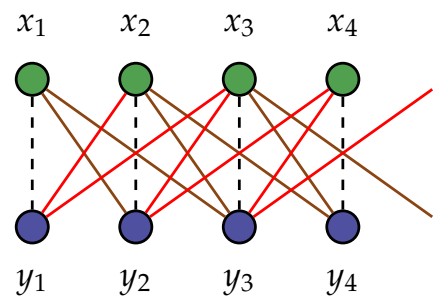

Figure 3.11: Situation in the proof of Theorem 3.25

We proceed by induction on $i$. If $i=2$, we need to prove that $y_{2} x_{1}$ and $x_{2} y_{1}$ are the first edges of $P$ and $Q$ respectively, and that $x_{1} y_{1}, x_{2} y_{2}$ are edges. Remember that $x_{1} \in P$. Obviously, $x_{1}$ is not an extreme of $P$. So, as $P$ is an ordered longest path, $x_{1}$ is the second vertex of $P$. Now we prove that $y_{2}$ is the first vertex of $P$. If $P$ starts in $y_{j}$ with $j>2$ then, as $y_{j} x_{1}$ and $x_{1} y_{1}$ are edges, $x_{1} y_{2}$ is an edge by Proposition 3.17 . Thus $\left(P-x_{1} y_{j}\right) \cdot x_{1} y_{2}$ is also a longest path, contradicting the choice of $P$. A similar reasoning shows that $x_{2} y_{1}$ is the first edge of $Q$. This implies, by Proposition 3.18 , that $x_{2} y_{2}$ is an edge, finishing the base case of the induction.

Now fix an $i>2$ and assume that both (a) and (b) are valid for all $j<i$. Then, by the induction hypothesis, $y_{i-1} x_{i-2}$ is the $(2 i-5)$-th edge of $P$. First, we prove that $x_{i-1}$ is the $(2 i-2)$-th vertex of $P$. Indeed, suppose that $x_{j}$ is the $(2 i-2)$-th vertex of $P$ with $j>i-1$. Let $P=P^{\prime} \cdot P^{\prime \prime}$, where $P^{\prime} \cap P^{\prime \prime}=\left\{x_{i-2}\right\}$. Then $y_{1} x_{1} y_{2} x_{2} \cdots y_{i-2} x_{i-2} \cdot P^{\prime \prime}$ is an ordered longest path that does not contain any vertex of $\left\{x_{i-1}, y_{i-1}\right\}$, a contradiction to Lemma 3.24. So $x_{i-1}$ is the $(2 i-2)$-th vertex of $P$. Now, we prove that $y_{i}$ is the $(2 i-3)$-th vertex of $P$. Suppose that $y_{j}$ is the $(2 i-3)$-th vertex of $P$ with $j>i$. Then, by Proposition 3.19 , as, by the induction hypothesis, $x_{i-1} y_{i-1}$ is an edge, $x_{i-2} y_{i}$ and $x_{i-1} y_{i}$ are edges. Now, $\left(P-x_{i-2} y_{j} x_{i-1}\right) \cdot x_{i-2} y_{i} x_{i-1}$ is also a longest path, contradicting the choice of $P$. A similar argument shows that $x_{i}$ is the $(2 i-3)$-th vertex of $Q$ and that $y_{i-1}$ is the $(2 i-2)$-th vertex of $Q$. This implies, by Proposition 3.18, that $x_{i} y_{i}$ is an edge, finishing the proof. 


\subsection{Remarks}

The problem of finding a minimum longest path transversal remains open for several well-studied graph classes. In this work, we proved that connected bipartite permutation graphs admit a transversal of cardinality one. This remains open for connected biconvex graphs and connected permutation graphs, well-known superclasses of bipartite permutation graphs. Even though our upper bound for $\operatorname{lpt}(G)$, when $G$ is a connected chordal graph, depends on $\omega(G)$, so far there are no examples of connected chordal graphs that require a transversal of cardinality greater than one. In this direction, one open problem is to look for such an example, if it exists, or to look for better bounds for $\operatorname{lpt}(G)$ when $G$ belongs to this graph class. It would be also interesting to generalize Theorem 3.14 for the class of substar graphs. In this class, only condition (ii) of the definition of full substar graphs is required to be satisfied. For more information about substar graphs, the reader may refer to Joos [Joo14].

The classical counterexample of Walther and Voss, and Zamfirescu (Figure 1.1) has treewidth three. (For a tree decomposition of such graph, see Figure 12 of [CEF $\left.{ }^{+} 17\right]$.) Hence, we have the following corollary of Theorem 3.10 .

Corollary 3.26. Let $\ell$ be the minimum integer such that $\operatorname{lpt}(G) \leq \ell$ for every connected partial 3-tree $G$. Then, $\ell \in\{2,3\}$. 


\section{Chapter 4}

\section{Transversals of Longest Cycles}

In this chapter we address transversals of longest cycles in some graph classes. Remember that lct is the minimum cardinality of a longest cycle transversal. We prove the following:

- $\operatorname{lct}(G)=1$ for every connected split graph $G$ (Section 4.1).

- $\operatorname{lct}(G) \leq \max \{1, \omega(G)-3\}$ for every 2-connected chordal graph $G$, where $\omega(G)$ is the cardinality of a maximum clique in $G$ (Section 4.2).

- $\operatorname{lct}(G) \leq k-1$ for every 2-connected partial $k$-tree $G$ (Section 4.3).

- $\operatorname{lct}(G)=1$ for every 2-connected partial 3-tree $G$ (Section 4.4.

Throughout this chapter, we use $L=L(G)$ for the length of a longest cycle in $G$. To recall the basic definitions on longest cycles, see Section 2.3 .

\subsection{Split Graphs}

We give a self-contained proof of a result given by Jobson et al. [JKLW16] that, in split graphs, all longest cycles intersect in a common vertex. Observe that in this section, the graphs considered are not necessarily 2-connected.

We say that a graph is split if there exists a partition of its vertex set into a clique and an independent set. More formally, a spliting of a graph $G$ is a partition $(K, S)$ of $V(G)$ such that $K$ is a clique and $S$ is an independent set (Figure 4.1). It is known that split graphs are chordal [Gol04, Theorem 6.3].

Proposition 4.1. Let $G$ be a split graph with two longest cycles $C$ and $D$. Then $V(C) \cap V(D) \neq \varnothing$.

Proof. Suppose by contradiction that $V(C) \cap V(D)=\varnothing$. As $|C| \geq 3, C$ must have at least two vertices in $K$. Indeed, suppose for a moment that $|V(C) \cap K| \leq 1$. Then $|V(C) \cap S| \geq|C|-1$, which implies that all vertices of $C$, except maybe one, form an independent set. Thus $C$ has at most two edges, a contradiction. Analogously, $D$ 


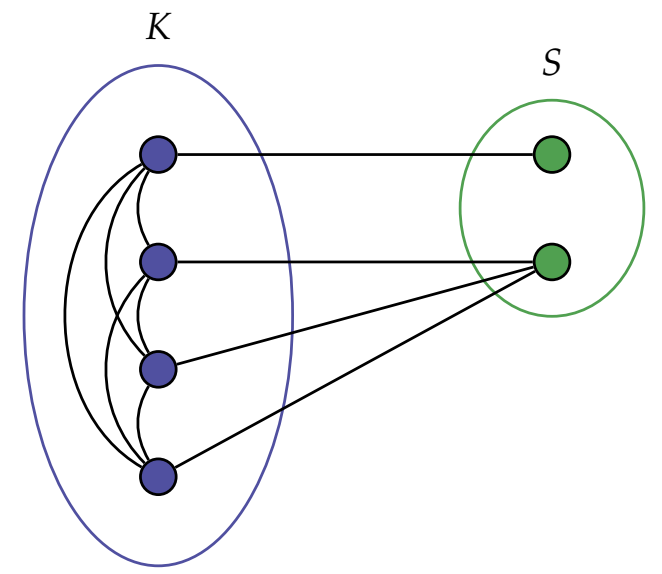

Figure 4.1: A split graph.

must have at least two vertices in $K$. Let $\{x, y\} \subseteq V(C) \cap K$ and $\{w, z\} \subseteq V(D) \cap K$. Let $C^{\prime}$ and $C^{\prime \prime}$ be the two $x y$-parts of $C$. Let $D^{\prime}$ and $D^{\prime \prime}$ be the two wz-parts of $D$. Then $C^{\prime} \cdot x w \cdot D^{\prime} \cdot z y$ and $C^{\prime \prime} \cdot x w \cdot D^{\prime \prime} \cdot z y$ are both cycles, one of them longer than $L$, a contradiction.

Proposition 4.2. Let $G$ be a split graph with a spliting $(K, S)$. If, for every pair of longest cycles $C$ and $D$ in $G$, either $V(C) \cap K \subseteq V(D) \cap K$ or $V(D) \cap K \subseteq V(C) \cap K$, then $\operatorname{lct}(G)=1$.

Proof. Let $C$ be a longest cycle in $G$ such that $|V(C) \cap K|$ is minimum. Let $D$ be a longest cycle in $G$. As $|V(C) \cap K|$ is minimum, then $V(C) \cap K \subseteq V(D) \cap K$. Hence every longest cycle in $G$ contains all vertices in $V(C) \cap K$. As $V(C) \cap K \neq \varnothing$, the proof follows.

Theorem 4.3. If $G$ is a split graph, then $\operatorname{lct}(G)=1$.

Proof. Suppose by contradiction that there exists a split graph $G$ such that $\operatorname{lct}(G)>1$. Let $(K, S)$ be a spliting of $G$. As $\operatorname{lct}(G)>1$, by Proposition 4.2 , there exists two longest cycles $C$ and $D$ in $G$ and two vertices $x \in V(C) \backslash V(D)$ and $y \in V(D) \backslash V(C)$.

Let $C_{x z}$ be a subpath of $C$ that starts at $x$, ends at a vertex $z$ of $C$ and is internally disjoint from $C$. Such a vertex exists by Proposition 4.1. We now consider two cases. First suppose that $z \in S$. Consider a neighbor $z^{\prime}$ of $z$ in $D$. Then $\left(D-z z^{\prime}\right) \cdot z^{\prime} x \cdot C_{x z}$ is a cycle longer than $L$, a contradiction (Figure 4.2(a)). Now suppose that $z \in K$. In this situation, consider a neighbor $z^{\prime}$ of $z$ in $D$, and a neighbor $z^{\prime \prime}$ of $z^{\prime}$ in $D$ different from $z$. Observe that $\left|C_{x z}\right|>1$. Otherwise, $\left(C-C_{x z}\right) \cdot x y z$ is a cycle longer than $L$, a contradiction. Then $\left(D-z z^{\prime} z^{\prime \prime}\right) \cdot C_{z x} \cdot x z^{\prime \prime}$ is a cycle longer than $L$, again a contradiction. 


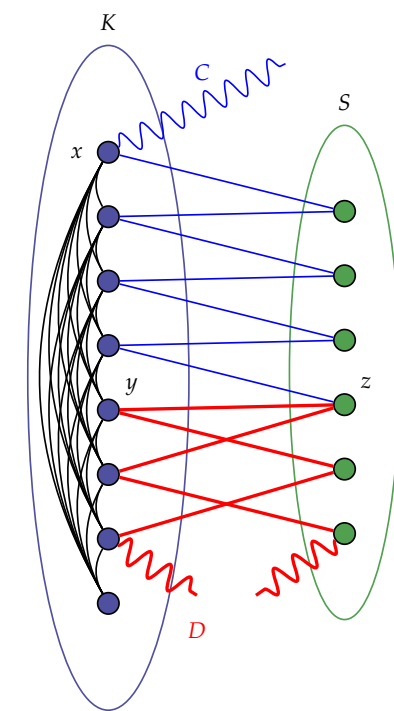

(a)

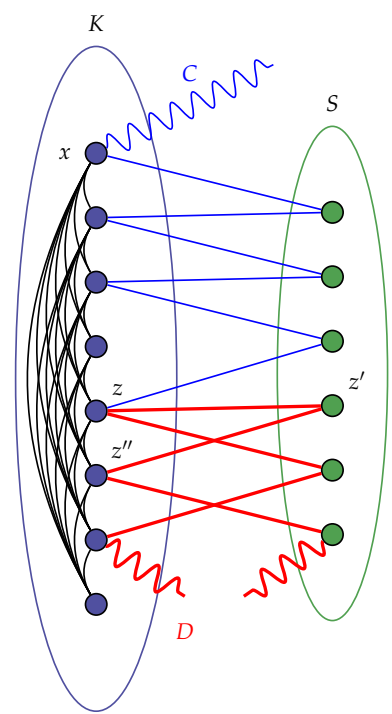

(b)

Figure 4.2: Cases in the proof of Theorem 4.3. (a) $z \in S$. (b) $z \in K$.

\subsection{Chordal Graphs}

In this section, we prove that $\operatorname{lct}(G) \leq \max \{1, \omega(G)-3\}$ for every 2-connected chordal graph $G$ (Theorem 4.13). As in our corresponding result for longest paths (Section 3.1), our main tool is to use the idea of the Helly Property for trees (Lemma 2.9) that was abstracted in Lemma 2.14 (Section 2.5). For this, again we use the corresponding clique tree of the chordal graph (Proposition 2.5). But, it is important to observe that, in chordal graphs, the bound for cycles is better than the bound for paths. This requires more work to obtain, for every node $t$ of the clique tree, our nonempty set $\mathscr{P}(t)$, which is finally obtained in Lemma 4.12 . For the proof of that Lemma, we need five previous lemmas. Roughly speaking, Lemma 4.4. Lemma 4.5 and Lemma 4.8 analyze the behaviour of several longest cycles that 2-intersects a clique, and Lemma 4.10 and Lemma 4.11 analyze the behaviour of several longest cycles that 3-intersects a clique.

We start our study of the behaviour of longest cycles that 2-intersect a maximal clique by proving a lemma valid for all 2-connected graphs. This lemma is similar to Lemma 3.1. but observe that the conclusion is stronger in the case of cycles.

Lemma 4.4. Let $G$ be a 2-connected graph with a clique $K$. Then every two longest cycles that 2-cross $K$ are K-equivalent.

Proof. Suppose by contradiction that there exists two longest cycles $C$ and $D$ that 2cross $K$ and $V(C) \cap K \neq V(D) \cap K$. Let $V(C) \cap K=\{a, b\}$ and $V(D) \cap K=\{c, d\}$. We may assume that either $\{a, b\}$ and $\{c, d\}$ are disjoint or $b=c$. Let $C^{\prime}$ and $C^{\prime \prime}$ be the two $a b$-parts of $C$. Let $D^{\prime}$ and $D^{\prime \prime}$ be the two $c d$-parts of $D$. As both $C$ and $D$ cross $K$, 
we may assume, without loss of generality, that $C^{\prime}$ is internally disjoint from $D^{\prime}$, and that $C^{\prime \prime}$ is internally disjoint from $D^{\prime \prime}$. Hence $C^{\prime} \cdot b c \cdot D^{\prime} \cdot d a$ and $C^{\prime \prime} \cdot b c \cdot D^{\prime \prime} \cdot d a$ are cycles, one of them longer than $L$, a contradiction (Figure 4.3 ).

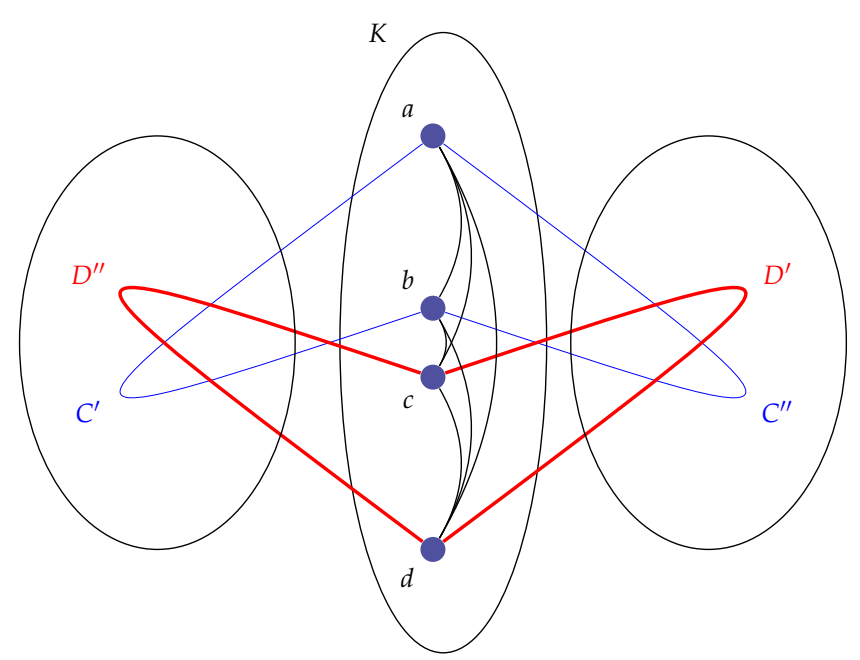

Figure 4.3: Situation in the proof of Lemma 4.4. Possibly $b=c$.

The next two lemmas analyse the case when there exists a longest cycle that 2-crosses some clique. Lemma 4.5 analyses the case when the clique has at least five vertices, and Lemma 4.8 analyses the case when the clique is small. Note that the former result holds for any graph. These two lemmas are analogous to Lemma 3.4.

Lemma 4.5. Let $G$ be a 2-connected graph. Let $K$ be a $k$-clique in $G$, with $k \geq 5$. If there exists a longest cycle that 2-crosses $K$ and $\operatorname{lct}(G)>\max \{1, \omega(G)-3\}$, then there is an $\ell$-attractor for $K$, with $\ell \leq 3$.

Proof. Let $C$ be a longest cycle that 2-crosses $K$ at $\{a, b\}$. Let $S$ be a subset of $K$ of cardinality $k-3$ that contains $\{a, b\}$. Such a subset exists because $k \geq 5$. As $\operatorname{lct}(G)>$ $\max \{1, \omega(G)-3\}=\omega(G)-3 \geq k-3$, there exists a longest cycle $\bar{D}$ that does not intersect $S$. This implies that $\bar{D}$ intersects $K$ at most three times, and that $\bar{D}$ does not intersect $\{a, b\}$. If $\bar{D}$ intersects $K$ at most once, then $\bar{D}$ is an $\ell$-attractor for $K$ with $\ell=0$ or 1 and we are done. Thus, we may assume that $\bar{D}$ intersects $K$ at least twice. Suppose that $\bar{D}$ 2-intersects $K$. Recall that $C 2$-crosses $K$ at $\{a, b\}$. Hence, by Lemma 4.4, every longest cycle that intersects $K$ at $V(\bar{D}) \cap K$ is fenced by $K$. We conclude that $\bar{D}$ is a 2-attractor for $K$.

Now suppose that $\bar{D}$ 3-intersects $K$ at $\{c, d, f\}$. Suppose for a moment that there exists a longest cycle $D$ that crosses $K$ at $\{c, d, f\}$. As $C$ and $D$ cross $K$, any of $\left\{D_{c d}, D_{d f}, D_{c f}\right\}$ is internally disjoint from at least one of $\left\{C^{\prime}, C^{\prime \prime}\right\}$, and any of $\left\{C^{\prime}, C^{\prime \prime}\right\}$ is internally disjoint from at least one of $\left\{D_{c d}, D_{d f}, D_{c f}\right\}$. Thus, we may assume, without 
loss of generality, that $C^{\prime}$ is internally disjoint from $D_{c d}$ and $D_{c f}$, and that $C^{\prime \prime}$ is internally disjoint from $D_{d f}$. Then, $C^{\prime} \cdot a d \cdot D_{d c} \cdot D_{c f} \cdot f b$ and $C^{\prime \prime} \cdot a d \cdot D_{d f} \cdot f b$ are both cycles, one of them longer than $L$, a contradiction (Figure 4.4). Hence, every longest cycle that intersects $K$ at $\{c, d, f\}$ is fenced by $K$. We conclude that $\bar{D}$ is a 3-attractor for $K$.

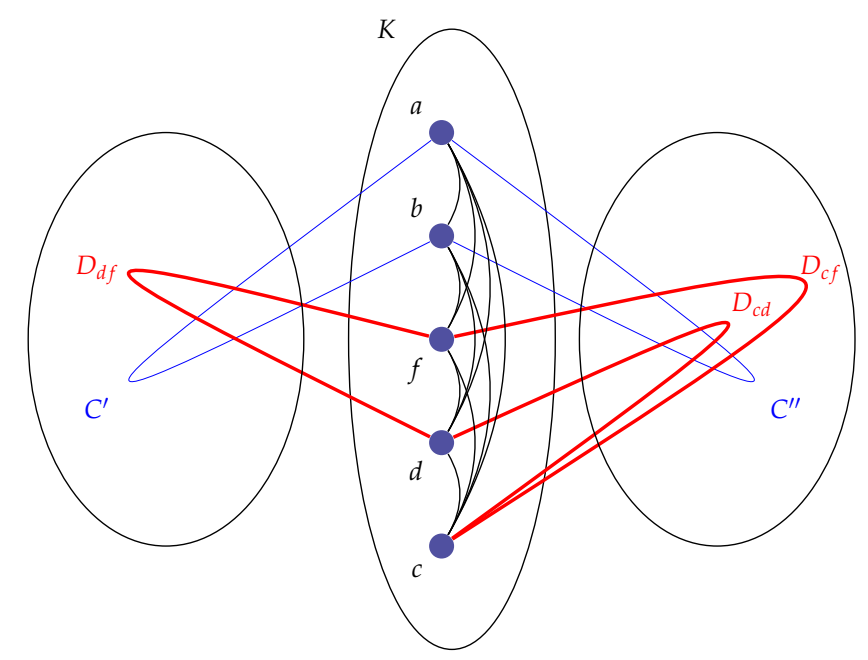

Figure 4.4: Situation in the proof of Lemma 4.5

We can extend the previous lemma for smaller cliques, but just for chordal graphs. Before that, we state two useful properties.

Proposition 4.6. Let $(T, \mathcal{V})$ be a tree decomposition of a graph $G$. Let $t \in V(T)$. Then $V_{t}$ separates, in $G$, two vertices in different branches of $T$ at $t$.

Proof. Let $u, v \in V(G) \backslash V_{t}$ such that $\operatorname{Branch}_{t}(u)=\operatorname{Branch}_{t}\left(t^{\prime}\right)$ and $\operatorname{Branch}_{t}(v)=$ $\operatorname{Branch}_{t}\left(t^{\prime \prime}\right)$, where $t^{\prime}$ and $t^{\prime \prime}$ are neighbors of $t$ in $T$. Observe that $\operatorname{Branch}_{t}\left(t^{\prime \prime}\right) \subseteq \operatorname{Branch}_{t^{\prime}}(t)$. Hence, $\operatorname{Branch}_{t}(v)=\operatorname{Branch}_{t^{\prime}}(t)$. By Proposition 2.7. $V_{t} \cap V_{t^{\prime}}$ separates, in $G, u \in \operatorname{Branch}_{t}\left(t^{\prime}\right)$ from $v \in \operatorname{Branch}_{t^{\prime}}(t)$.

Proposition 4.7. Let $G$ be a chordal graph and $K$ be a maximal clique in $G$. Let $\mathscr{C}$ be a collection of at least two paths fenced by $K$, that 2-intersect $K$ and whose extremes are in $K$. If $K \subseteq \bigcup_{P \in \mathscr{C}} V(P)$, then there exist two paths $P$ and $Q$ in $\mathscr{C}$, such that $P \nsim_{K} Q$.

Proof. Let $(T, \mathcal{V})$ be a clique tree of $G$. As $K$ is a maximal clique in $G$, there exists $t \in V(T)$ such that $V_{t}=K$. Suppose for a contradiction that $P \sim_{K} Q$ for every $P, Q \in \mathscr{C}$. Observe that no path $P \in \mathscr{C}$ has an edge in $K$, otherwise, $P$ would consist of only this edge, because $P$ 2-intersects $K$ and has its extremes in $K$, and thus $P \nsim_{K} Q$ for every other path $Q \in \mathscr{C}$. This implies that $\operatorname{Branch}_{t}(P)=\operatorname{Branch}_{t}(Q)$ for every $P, Q \in \mathscr{C}$. Indeed, let $P, Q \in \mathscr{C}$. As $P \sim_{K} Q$, there exists $u \in V(P)$ and $v \in V(Q)$ such that $u$ and $v$ are in the same component of $G-V_{t}$, hence $\operatorname{Branch}_{t}(u)=\operatorname{Branch}_{t}(v)$ by Proposition 4.6. So, by 
Proposition 2.13, there exists an edge $t t^{\prime} \in E(G)$ such that $\operatorname{Branch}_{t}(P)=\operatorname{Branch}_{t}\left(t^{\prime}\right)$ for every $P \in \mathscr{C}$. Therefore, by Proposition 2.8, $V_{t} \cap V(P) \subseteq V_{t^{\prime}}$ for every $P \in \mathscr{C}$. Hence, $V_{t} \subseteq V_{t^{\prime}}$, a contradiction because $(T, \mathcal{V})$ is a clique tree.

Lemma 4.8. Let $G$ be a 2-connected chordal graph with a maximal clique $K$ such that $|K| \leq 4$. If there exists a longest cycle that 2-crosses $K$, then either $\operatorname{lct}(G)=1$ or there is an $\ell$-attractor for $K$, with $\ell \leq 2$.

Proof. Let $C$ be a longest cycle that 2-crosses $K$, say, at $\{a, b\}$. Suppose that $\operatorname{lct}(G)>1$. Then there exists a longest cycle $D$ in $G$ that does not contain $a$. If $D$ intersects $K$ at most once, then $D$ is an $\ell$-attractor for $K$ with $\ell=0$ or 1 and we are done. If $D$ 2-intersects $K$, then, by Lemma 4.4, all cycles that intersect $K$ at $V(D) \cap K$ are fenced by $K$. We conclude that $D$ is a 2-attractor for $K$, so we are done.

It remains to consider the case in which $|K|=4$ and $D$ 3-intersects $K$, say, at $\{b, c, d\}$. Let $C^{\prime}$ and $C^{\prime \prime}$ be the two $a b$-parts of $C$. Applying Proposition 4.7 to $\mathscr{C}=\left\{C^{\prime}, D_{c d}\right\}$ and to $\mathscr{C}=\left\{C^{\prime \prime}, D_{c d}\right\}$, we conclude that $C^{\prime} \nsim_{K} D_{c d}$ and $C^{\prime \prime} \nsim_{K} D_{c d}$. As $C$ and $D$ intersect each other in a vertex other than $b$ (because $G$ is 2-connected), we may assume without loss of generality that $C^{\prime} \sim_{K} D_{b d}$. By Proposition 4.7 again, applied to $\mathscr{C}=\left\{C^{\prime}, D_{b c}, D_{b d}\right\}$, we conclude that $D_{b c} \nsim_{K} D_{b d} \sim_{K} C^{\prime}$. But then $C^{\prime} \cdot D_{b c} \cdot D_{c d} \cdot d a$ and $C^{\prime \prime} \cdot D_{b d} \cdot d a$ are cycles, one of them longer than $L$, a contradiction.

Now we study the behaviour of longest cycles that 3-cross a bag. Recall that, given a longest cycle $C$ that 3 -crosses $K$ at $\{a, b, c\}$, we say that $a$ is a $C$-breaking vertex if $C_{a b} \nsim_{K} C_{a c}$ (Section 2.3).

Proposition 4.9. Let $G$ be a graph and $C$ be a cycle in $G$. Let $K \subseteq V(G)$. Let $\Delta$ be a triangle in $K$. If $C$ 3-crosses $K$ at $\Delta$, then $\Delta$ has at least two $C$-breaking vertices.

Proof. Let $\Delta=a b c$. As $C$ crosses $K$, at least two of $\left\{C_{a b}, C_{a c}, C_{b c}\right\}$ are in different components of $G-K$. Suppose without loss of generality that $C_{a b} \nsim_{K} C_{a c}$. Then $a$ is a $C$-breaking vertex. Also, either $C_{b c} \nsim_{K} C_{a b}$ or $C_{b c} \nsim_{K} C_{a c}$. Hence one of $\{b, c\}$ is also a C-breaking vertex.

The next two lemmas analyse the case when there exists a longest cycle that 3-crosses some clique. Lemma 4.10 analyses the case when the clique has at least five vertices, and Lemma 4.11 analyses the case when the clique is small. Note that the former result holds for any graph.

Lemma 4.10. Let $G$ be a 2-connected graph with a clique $K$ such that $|K| \geq 5$. If, for every triangle $\Delta \subseteq K$, there exists a longest cycle that 3-intersects $K$ at $\Delta$, then at least one of these cycles is a 3-attractor for $K$. 
Proof. Suppose by contradiction that none of these cycles is a 3-attractor for $K$. Then, for every triangle $\Delta \subseteq K$ there exists a longest cycle $C_{\Delta}$ that 3-crosses $K$ at $\Delta$. By Proposition 4.9. for every $\Delta$ in $K, \Delta$ has at least two $C_{\Delta}$-breaking vertices. As there are $\left(\begin{array}{c}|K| \\ 3\end{array}\right)$ triangles in $K$, by pigeonhole principle, there exists a vertex $v \in K$ such that $v$ is a breaking vertex for at least $\frac{(|K|-1)(|K|-2)}{3}$ of the triangles incident to $v$. Observe that there exists at most $|K|-2$ triangles incident to $v$ that share a vertex other than $v$. Thus, as $|K| \geq 5$, there exists two edge-disjoint triangles incident to $v$ such that $v$ is a breaking vertex for both of them. Let $v a b$ and $v c d$ be such triangles, and let $C$ and $D$ be the corresponding cycles respectively.

As $v$ breaks both $C$ and $D$, without loss of generality we may assume that $C_{v a} \nsim_{K} D_{v c}$ and that $C_{v b} \nsim_{K} D_{v d}$. Also, there exists a part $X \in\left\{D_{v c}, D_{v d}\right\}$ such that $C_{a b} \nsim_{K} X$ and a part $Y \in\left\{C_{v a}, C_{v b}\right\}$ such that $D_{c d} \nsim_{K} Y$. Without loss of generality, we may suppose that $C_{a b} \nsim_{K} D_{v d}$. If $D_{c d} \nsim_{K} C_{v a}$ then $D_{v c} \cdot D_{c d} \cdot d a \cdot C_{a v}$ and $D_{d v} \cdot C_{v b} \cdot C_{b a} \cdot a d$ are cycles, a contradiction (Figure 4.5 (a)). So $D_{c d} \sim_{K} C_{v a}$, and $D_{c d} \nsim_{K} C_{v b}$. If $C_{a b} \nsim_{K} D_{c d}$ then $C_{v a} \cdot a c \cdot D_{c v}$ and $C_{v b} \cdot C_{b a} \cdot a c \cdot D_{c d} \cdot D_{d v}$ are cycles, a contradiction (Figure 4.5(b)). So, $C_{a b} \sim_{K} D_{c d}$. As $D_{c d} \sim_{K} C_{v a}$, we conclude that $C_{a b} \sim_{K} C_{v a}$. As $C_{v a} \nsim_{K} D_{v c}$, we conclude that $C_{a b} \nsim_{K} D_{v c}$. Then, $C_{v a} \cdot C_{a b} \cdot b c \cdot D_{c v}$ and $C_{v b} \cdot b c \cdot D_{c d} \cdot D_{d v}$ are both cycles, one of them longer than $L$, again a contradiction (Figure $4.5(\mathrm{c})$ ).

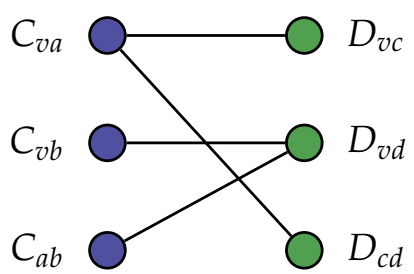

(a)

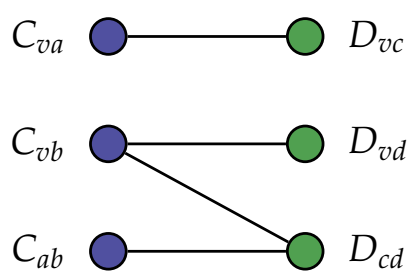

(b)

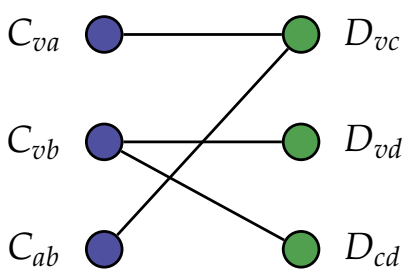

(c)

Figure 4.5: Each bipartite graph represents the situation of the cycles $C$ and $D$ in the proof of Lemma 4.10. Each side of the bipartition has three vertices that represent the parts of each cycle. There is a straight edge in the graph if the corresponding parts, say $X$ and $Y$, are such that $X \nsim_{K} Y$.

We can extend the previous lemma for smaller cliques, but just for chordal graphs.

Lemma 4.11. Let $G$ be a 2-connected chordal graph with a maximal clique $K$ such that $|K|=4$. If for every triangle $\Delta$ in $K$ there exists a longest cycle that 3-intersects $K$ at $\Delta$, then either $\operatorname{lct}(G)=1$ or there is an $\ell$-attractor for $K$ with $\ell \leq 3$.

Proof. Let $(T, \mathcal{V})$ be a clique tree of $G$. Suppose that $K$ has no 3-attractor. Then, for every triangle $\Delta$ in $K$, there exists a longest cycle that 3-crosses $K$ at $\Delta$. By Proposition 4.9. for every $\Delta$ in $K, \Delta$ has at least two breaking vertices. As $|K|=4$, there are four such 
triangles. By the pigeonhole principle, there exists a vertex $a \in K$ such that $a$ is a breaking vertex for at least two of the triangles incident to $a$. Let $a b d$ and acd be these two triangles. Let $C$ and $D$ be the corresponding longest cycles respectively. Hence,

$$
C_{a b} \nsim_{K} C_{a d} \text { and } D_{a c} \nsim_{K} D_{a d}
$$

Also, by Proposition 4.7

$$
C_{a b} \nsim_{K} D_{c d} \text { and } C_{b d} \nsim_{K} D_{a c} \text {. }
$$

By (4.1) either $C_{a b} \nsim_{K} D_{a d}$ and $C_{a d} \nsim_{K} D_{a c}$, or $C_{a b} \nsim_{K} D_{a c}$ and $C_{a d} \nsim_{K} D_{a d}$. In the first case, $C_{b a} \cdot D_{a d} \cdot D_{d c} \cdot c b$ and $D_{a c} \cdot c b \cdot C_{b d} \cdot C_{d a}$ are cycles, one of them longer than $L, a$ contradiction (Figure 4.6(a)).

In the second case, $C_{b d} \nsim_{K} D_{c d}$. Indeed, suppose for a moment that $C_{b d} \sim_{K} D_{c d}$. Then, by Proposition 4.7, with $\mathscr{C}=\left\{C_{b d}, D_{c d}, C_{a d}\right\}$ we have that $C_{a d} \nsim_{K} D_{c d}$. And by the same proposition, with $\mathscr{C}=\left\{C_{b d}, D_{c d}, D_{a d}\right\}$, we have that $C_{b d} \nsim_{K} D_{a d}$, so $C_{b a} \cdot C_{a d} \cdot D_{d c} \cdot c b$ and $D_{c a} \cdot D_{a d} \cdot C_{d b} \cdot b c$ are cycles, one of them longer than $L$, a contradiction (Figure 4.6(b)). Hence, $C_{b d} \nsim_{K} D_{c d}$. Thus, $C_{a d} \cdot D_{a d}$ and $C_{a b} \cdot C_{b d} \cdot D_{d c} \cdot D_{c a}$ are cycles (Figure 4.6(c)). But then, $C_{a d} \cdot D_{a d}$ is a longest cycle that 2-crosses $V_{t}$, and we conclude the proof by applying Lemma 4.8 .

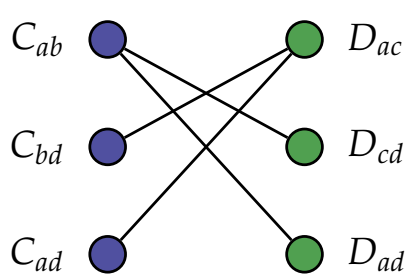

(a)

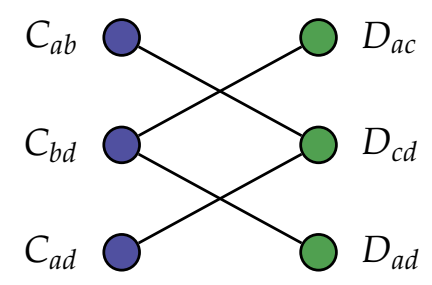

(b)

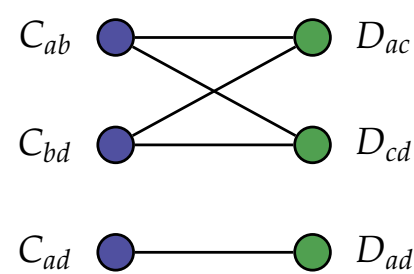

(c)

Figure 4.6: Each bipartite graph represents the situation of the cycles $C$ and $D$ in the proof of Lemma 4.11. Each side of the bipartition has three vertices that represent the parts of each cycle. There is a straight edge in the graph if the corresponding parts, say $X$ and $Y$, are such that $X \nsim_{K} Y$.

The following lemma synthesizes the previous four lemmas.

Lemma 4.12. Let $G$ be a 2-connected chordal graph such that $\operatorname{lct}(G)>\max \{1, \omega(G)-3\}$. Let $k$ be an integer with $k \geq 2$. For each clique of cardinality $k$ in $G$, there exists an $\ell$-attractor, with $\ell \leq \min \{3, k-1\}$.

Proof. Let $K$ be a clique in $G$ and $k \geq 2$ be its cardinality. Then, for every set of $\max \{1, \omega(G)-3\}$ vertices in $K$, there exists a longest cycle that does not intersect that set. If one of these cycles intersects $K$ at most once, then we are done. Indeed, 
such cycle could be an $\ell$-attractor for $K$, with $\ell=0$ or 1 . Hence, we may assume that every such cycle intersects $K$ at least twice. Which implies, as $\max \{1, \omega(G)-3\} \geq 1$, that $k \geq 3$. Suppose for a moment that one of these cycles, say $C, 2$-intersects $K$. If $C$, or any longest cycle equivalent to $C, 2$-crosses $K$, then we are finished by Lemma 4.5 and Lemma 4.8 . Indeed, if $k \geq 5$ then, by Lemma 4.5 , there is an $\ell$-attractor for $K$ with $\ell \leq 3 \leq \min \{3, k-1\}$ and we are done. If $3 \leq k \leq 4$ then, by Lemma 4.8 , there is an $\ell$-attractor for $K$ with $\ell \leq 2 \leq \min \{3, k-1\}$ and we are done. So, all longest cycles equivalent to $C$ are fenced by $K$ and thus $C$ is an $\ell$-attractor for $K$ with $\ell=2 \leq \min \{3, k-1\}$. Thus, as $k-\max \{1, \omega(G)-3\} \leq 3$, it remains to consider the case in which each of these cycles 3 -intersects $K$, which implies, as $\max \{1, \omega(G)-3\} \geq 1$, that $k \geq 4$. By Lemma 4.10 and Lemma 4.11 there is an $\ell$-attractor for $K$ with $\ell \leq 3 \leq \min \{3, k-1\}$ and we are done.

Finally, we obtain our main result.

Theorem 4.13. For every 2-connected chordal graph $G, \operatorname{lct}(G) \leq \max \{1, \omega(G)-3\}$.

Proof. Let $(T, \mathcal{V})$ be a clique tree of $G$. If one of such cliques has cardinality one, $|V(G)|=1$ and we are done. Thus, every clique in $\mathcal{V}$ has cardinality at least two. For every $t \in V(T)$, let $\mathscr{P}(t)$ be the set of longest cycles in $G$ which are $\ell$-attractors for $V_{t}$, with $\ell \leq \min \left\{3,\left|V_{t}\right|-1\right\}$. Suppose by contradiction that $\operatorname{lct}(G)>\max \{1, \omega(G)-3\}$. Then, $\mathscr{P}(t) \neq \varnothing$ for every $t \in V(T)$ by Lemma 4.12 . Observe that, as $V_{t}$ is a clique, any cycle in $\mathscr{P}(t)$ has no edges in $G\left[V_{t}\right]$. Otherwise, such cycle will contain all vertices of $V_{t}$, a contradiction to the fact that $\ell \leq \min \left\{3,\left|V_{t}\right|-1\right\}$. This implies that, for any $t \in V(T)$, no cycle in $\mathscr{P}(t)$ is contained in $G\left[V_{t}\right]$. Thus, by Lemma 2.14, there exists an edge $t t^{\prime} \in E(T)$ and two cycles $C \in \mathscr{P}(t)$ and $D \in \mathscr{P}\left(t^{\prime}\right)$ such that $\operatorname{Branch}_{t}(C)=\operatorname{Branch}_{t}\left(t^{\prime}\right), \operatorname{Branch}_{t^{\prime}}(D)=\operatorname{Branch}_{t^{\prime}}(t), C$ is an $\ell$-attractor for $V_{t}$, with $\ell \leq \min \left\{3,\left|V_{t}\right|-1\right\}$, and $D$ is an $\ell^{\prime}$-attractor for $V_{t^{\prime}}$, with $\ell^{\prime} \leq \min \left\{3,\left|V_{t^{\prime}}\right|-1\right\}$.

Let $u \in V_{t} \backslash V_{t^{\prime}}$. Suppose for a moment that $u \in V(C)$. Let $u v \in E(C)$. By Proposition 2.7. $\operatorname{Branch}_{t}(v) \neq \operatorname{Branch}_{t}\left(t^{\prime}\right)$, so $v \in V_{t}$. This implies that $u v$ is an edge in $V_{t}$, a contradiction. Thus, $\left(V_{t} \backslash V_{t^{\prime}}\right) \cap V(C)=\varnothing$ and $\left(V_{t^{\prime}} \backslash V_{t}\right) \cap V(D)=\varnothing$, which implies that $V_{t} \cap V_{t^{\prime}}$ separates a vertex in $V(C) \backslash V_{t}$ from a vertex in $V(D) \backslash V_{t^{\prime}}$.

Suppose for a moment that $\left|V_{t} \cap V_{t^{\prime}}\right| \leq \omega(G)-2$. As $\operatorname{lct}(G)>\max \{1, \omega(G)-3\}$, there exists a longest cycle $R$ that contains at most one vertex of $V_{t} \cap V_{t^{\prime}}$. This leads to a contradiction, because, as $G$ is 2-connected, $R$ must intersect a vertex in $V(C) \backslash V_{t}$ and a vertex in $V(D) \backslash V_{t^{\prime}}$. Hence $\left|V_{t} \cap V_{t^{\prime}}\right| \geq \omega(G)-1$. Moreover, as both $V_{t}$ and $V_{t^{\prime}}$ are maximal (and different), we conclude that $\left|V_{t}\right|=\left|V_{t^{\prime}}\right|=\omega(G)$ and $\left|V_{t} \cap V_{t^{\prime}}\right|=\omega(G)-1$. Let $\{u\}=V_{t} \backslash V_{t^{\prime}}$ and $\{w\}=V_{t^{\prime}} \backslash V_{t}$.

Case 1: Both $C$ and $D$ 3-intersect $V_{t}$ and $V_{t^{\prime}}$, respectively. 
Let $V(C) \cap V_{t}=\{a, b, c\}$. Consider the case when $V(D) \cap V_{t^{\prime}}=\{a, b, c\}$. Let $C_{a b}, C_{b c}, C_{a c}$ be the corresponding $a b c$-parts of $C$. Let $D_{a b}, D_{b c}, D_{a c}$ be the corresponding $a b c$-parts of $D$. By pigeonhole principle, we may assume, without loss of generality, that $w \notin C_{a b}$ and that $u \notin D_{a b}$. As $\left(C-C_{a b}\right) \cdot D_{a b}$ and $\left(D-D_{a b}\right) \cdot C_{a b}$ are cycles, $\left|C_{a b}\right|=\left|D_{a b}\right|$ and both are longest cycles. Hence, $\left(C-C_{a b}\right) \cdot D_{a b}$ is a longest cycle that 3-crosses $V_{t}$ at $V(C) \cap V_{t}$, a contradiction to the fact that $C$ is an attractor for $V_{t}$ (Figure 4.7(a)). Now suppose that $V(D) \cap V_{t^{\prime}}=\{b, c, d\}$, with $d \neq a$. Then, $C_{b c} \cdot C_{c a} \cdot a d \cdot D_{d b}$ and $D_{b c} \cdot D_{c d} \cdot d a \cdot C_{a b}$ are cycles, one of them longer than $L$, a contradiction.

Case 2: Both $C$ and $D$ 2-intersect $V_{t}$ and $V_{t^{\prime}}$, respectively.

As $G$ is 2-connected, we may assume that $V(C) \cap V_{t}=V(D) \cap V_{t^{\prime}}=\{a, b\}$. Let $C^{\prime}$ and $C^{\prime \prime}$ be the two $a b$-parts of $C$. Let $D^{\prime}$ and $D^{\prime \prime}$ be the two $a b$-parts of $D$. As $\left(C-C^{\prime}\right) \cdot D^{\prime},\left(C-C^{\prime}\right) \cdot D^{\prime \prime}, \quad\left(D-D^{\prime}\right) \cdot C^{\prime}$ and $\left(D-D^{\prime}\right) \cdot C^{\prime \prime}$ are cycles, $\left|C^{\prime}\right|=\left|C^{\prime \prime}\right|=\left|D^{\prime}\right|=\left|D^{\prime \prime}\right|=L / 2$. Without loss of generality we may assume that $u \notin V\left(D^{\prime}\right)$. Hence, $D^{\prime} \cdot C^{\prime}$ is a longest cycle that 2-crosses $V_{t}$ at $V(C) \cap V_{t}$, a contradiction to the fact that $C$ is an attractor for $V_{t}$ (Figure $4.7(b)$ ).

Case 3: $C$ 3-intersects $V_{t}$ and $D$ 2-intersects $V_{t^{\prime}}$.

As $G$ is 2-connected, we may assume that $V(C) \cap V_{t}=\{a, b, c\}$ and that $V(D) \cap V_{t^{\prime}}=\{a, b\}$. Let $C_{a b}, C_{b c}, C_{a c}$ be the corresponding abc-parts of $C$. Let $D^{\prime}$ and $D^{\prime \prime}$ be the two $a b$-parts of $D$. Without loss of generality, we may assume that $u \notin V\left(D^{\prime}\right)$. Hence, $\left(C-C_{a b}\right) \cdot D^{\prime}$ is a longest cycle that 3-crosses $V_{t}$ at $V(C) \cap V_{t}$, a contradiction to the fact that $C$ is an attractor for $V_{t}$ (Figure 4.7(c)).

This concludes the proof.

The previous theorem implies the following results.

Corollary 4.14. If $G$ is a $k$-tree, with $k>2$, then $\operatorname{lct}(G) \leq k-2$.

Corollary 4.15. All longest cycles intersect in 2-trees, 3-trees, and in 2-connected chordal planar graphs.

\subsection{Partial $k$-Trees}

Recall that, by Proposition 2.2. partial $k$-trees are exactly those graphs with treewidth at most $k$. As in the case of paths (Section 3.2), it is easy to show an upper bound of $k+1$ for $\operatorname{lct}(G)$ when $G$ is a partial $k$-tree, by giving an analogous proof of Lemma 3.8 for cycles instead of paths. In this section, we improve this bound and show that $\operatorname{lct}(G) \leq k-1$ when $G$ is a 2-connected partial $k$-tree (Theorem 4.18).

We begin by showing a useful lemma.

Lemma 4.16. Let $G$ be a 2-connected graph with treewidth at least two. Let $(T, \mathcal{V})$ be a full tree decomposition of $G$. Let $V_{t} \in \mathcal{V}$. If $\operatorname{lct}(G)>\left|V_{t}\right|-2$, then $V_{t}$ has an $\ell$-attractor, with $\ell \leq 2$. 


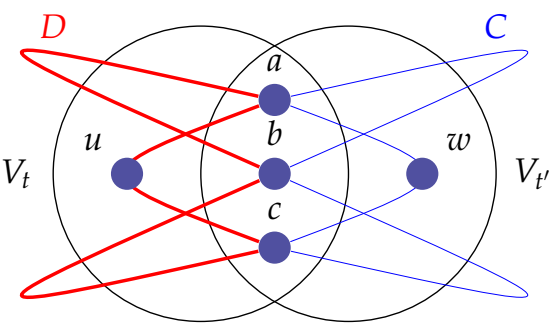

(a)

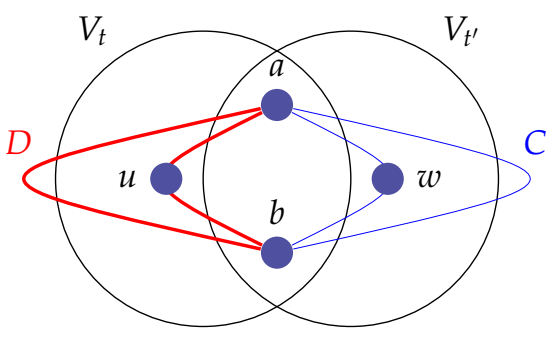

(b)

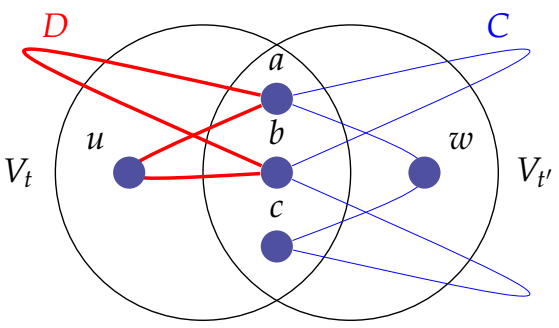

(c)

Figure 4.7: Cases in the proof of Theorem 4.13 .

Proof. As lct $(G)>\left|V_{t}\right|-2$, for every subset of $V_{t}$ of cardinality $\left|V_{t}\right|-2$, there exists a longest cycle that does not contain any vertex of it. If any of these cycles intersects $V_{t}$ at most once, then there is an $\ell$-attractor for $V_{t}$ with $\ell \leq 1$ and we are done. Hence, every such cycle 2-intersects $V_{t}$. That is, for every pair of vertices in $V_{t}$, there exists a longest cycle that 2-intersects $V_{t}$ at such pair. Suppose by contradiction that $V_{t}$ has no $\ell$-attractor, with $\ell \leq 2$. Then, for every pair of vertices in $V_{t}$, there exists a longest cycle that 2-crosses $V_{t}$ at such pair. Observe that it cannot be the case that all such cycles contain an edge of $V_{t}$. Hence, there exists a longest cycle $C$ that 2-crosses $V_{t}$ at $\{a, b\}$ such that $a b \notin E(C)$. Let $C^{\prime}$ and $C^{\prime \prime}$ be the two $a b$-parts of $C$. As both $C^{\prime}$ and $C^{\prime \prime}$ are fenced by $V_{t}$, by Proposition 2.13, there exists two nodes $t^{\prime}, t^{\prime \prime}$, neighbors of $t$ in $T$, such that $\operatorname{Branch}_{t}\left(C^{\prime}\right)=\operatorname{Branch}_{t}\left(t^{\prime}\right)$ and $\operatorname{Branch}_{t}\left(C^{\prime \prime}\right)=\operatorname{Branch}_{t}\left(t^{\prime \prime}\right)$, where possibly $t^{\prime}=t^{\prime \prime}$.

Case 1: $V_{t} \cap V_{t^{\prime}}=V_{t} \cap V_{t^{\prime \prime}}$. As $\left|V_{t} \cap V_{t^{\prime}}\right|=\left|V_{t}\right|-1$, there exists a vertex $x \in V_{t} \backslash V_{t^{\prime}}=V_{t} \backslash V_{t^{\prime \prime}}$. Let $y$ be an arbitrary vertex in $V_{t}$ different from $x$. Let $D$ be a longest cycle that 2-crosses $V_{t}$ at $\{x, y\}$. Then, as $C$ and $D$ intersect each other in at least two vertices, there exists a $x y$-part of $D$, called it $D^{\prime}$, such that either $\operatorname{Branch}_{t}\left(D^{\prime}\right)=\operatorname{Branch}_{t}\left(t^{\prime}\right)$ or $\operatorname{Branch}_{t}\left(D^{\prime}\right)=\operatorname{Branch}_{t}\left(t^{\prime \prime}\right)$. If the former is true, then, by Proposition 2.8. $\{x, y\}=V_{t} \cap V\left(D^{\prime}\right) \subseteq V_{t^{\prime}}$, a contradiction to the fact that $x \in V_{t} \backslash V_{t^{\prime}}$. If the later is true, then, by Proposition 2.8, $\{x, y\}=V_{t} \cap V\left(D^{\prime}\right) \subseteq V_{t^{\prime \prime}}$, a contradiction to the fact that $x \in V_{t} \backslash V_{t^{\prime \prime}}$.

Case 2: $V_{t} \cap V_{t^{\prime}} \neq V_{t} \cap V_{t^{\prime \prime}}$. As $\left|V_{t} \cap V_{t^{\prime}}\right|=\left|V_{t} \cap V_{t^{\prime \prime}}\right|$, there exists a vertex $x \in\left(V_{t} \cap V_{t^{\prime}}\right) \backslash V_{t^{\prime \prime}}$ and a vertex $y \in\left(V_{t} \cap V_{t^{\prime \prime}}\right) \backslash V_{t^{\prime}}$. Let $D$ be a longest cycle that 2-crosses $V_{t}$ at $\{x, y\}$. Then, as $C$ and $D$ intersect in at least two vertices, there 
exists a $x y$-part of $D$, called it $D^{\prime}$, such that either $\operatorname{Branch}_{t}\left(D^{\prime}\right)=\operatorname{Branch}_{t}\left(t^{\prime}\right)$ or $\operatorname{Branch}_{t}\left(D^{\prime}\right)=\operatorname{Branch}_{t}\left(t^{\prime \prime}\right)$. If the former is true, then by Proposition 2.8. $\{x, y\}=V_{t} \cap V\left(D^{\prime}\right) \subseteq V_{t^{\prime}}$, a contradiction to the fact that $y \in\left(V_{t} \cap V_{t^{\prime \prime}}\right) \backslash V_{t^{\prime}}$. If the later is true, then by Proposition 2.8, $\{x, y\}=V_{t} \cap V\left(D^{\prime}\right) \subseteq V_{t^{\prime \prime}}$, a contradiction to the fact that $x \in\left(V_{t} \cap V_{t^{\prime}}\right) \backslash V_{t^{\prime \prime}}$.

Proposition 4.17. Let $t t^{\prime}$ be an edge of $T$ and let $C$ be a cycle in $G$. If $C$ has a vertex in $V_{t} \backslash V_{t^{\prime}}$ and a vertex in $\operatorname{Branch}_{t}\left(t^{\prime}\right)$, then $\left|V(C) \cap V_{t}\right| \geq 3$.

Proof. Let $u, v \in C$ such that $u \in V_{t} \backslash V_{t^{\prime}}$ and $v \in \operatorname{Branch}_{t}\left(t^{\prime}\right)$. Then $u \in \operatorname{Branch}_{t^{\prime}}(t)$ because $t \in \operatorname{Branch}_{t^{\prime}}(t)$. By Proposition 2.7, $V_{t} \cap V_{t^{\prime}}$ separates $u$ from $v$. As $u, v \in C$, there are two internally disjoint paths in $G$ that joins $u$ and $v$. As neither $u$ nor $v$ belong to $V_{t} \cap V_{t^{\prime}}$, this set must intersect at least twice these two disjoint paths, so $\left|V(C) \cap V_{t} \cap V_{t^{\prime}}\right| \geq 2$, and as $u \in V_{t} \backslash V_{t^{\prime}}$, we conclude that $\left|V_{t} \cap V(C)\right| \geq 3$.

Finally, we prove our main theorem.

Theorem 4.18. For every 2-connected partial $k$-tree $G$, we have $\operatorname{lct}(G) \leq k-1$.

Proof. Let $(T, \mathcal{V})$ be a full tree decomposition of $G$. For every $t \in V(T)$, let $\mathscr{P}(t)$ be the set of longest cycles in $G$ such that, for every $C \in \mathscr{P}(t), C$ is an $\ell$-attractor for $V_{t}$, with $\ell \leq 2$. Suppose by contradiction that $\operatorname{lct}(G)>k-1$. As $G$ is 2-connected, it is not a forest, so $k \geq 2$. Then, as $\left|V_{t}\right|=k+1$, by Lemma 4.16, $\mathscr{P}(t) \neq \varnothing$ for every $t \in V(T)$. Thus, by Lemma 2.14, there exists an edge $t t^{\prime} \in E(T)$ and two longest cycles $C$ and $D$ in $G$ such that $\operatorname{Branch}_{t}(C)=\operatorname{Branch}_{t}\left(t^{\prime}\right), \operatorname{Branch}_{t^{\prime}}(D)=\operatorname{Branch}_{t^{\prime}}(t), C$ is an $\ell$-attractor for $V_{t}$, with $\ell \leq 2$, and $D$ is an $\ell^{\prime}$-attractor for $V_{t^{\prime}}$, with $\ell^{\prime} \leq 2$.

Note that the bags containing vertices of $C$ are only in $\operatorname{Branch}_{t}\left(t^{\prime}\right) \cup\{t\}$, and that the bags containing vertices of $D$ are only in Branch $_{t^{\prime}}(t) \cup\left\{t^{\prime}\right\}$. As $\operatorname{Branch}_{t}\left(t^{\prime}\right)$ and Branch $_{t^{\prime}}(t)$ are disjoint, $V(C) \cap V(D) \subseteq V_{t} \cup V_{t^{\prime}}$. Let $V_{t}=\{a, b, c, u\}$ and $V_{t^{\prime}}=\{a, b, c, w\}$. Suppose for a moment that $u \in V(C)$. As $C$ intersects $V_{t}$ at most twice, there exists a vertex $v \in V(C) \backslash V_{t}$. As $\operatorname{Branch}_{t}(C)=\operatorname{Branch}_{t}\left(t^{\prime}\right), v \in \operatorname{Branch}_{t}\left(t^{\prime}\right)$. But then, $\left|V(C) \cap V_{t}\right| \geq 3$ by Proposition 4.17 , a contradiction.

So $u \notin V(C)$. Analogously, we can conclude that $w \notin V(D)$ and therefore $V(C) \cap V(D) \subseteq V_{t} \cap V_{t^{\prime}}$. As $G$ is 2-connected, this implies that $\left|V(C) \cap V_{t}\right| \geq 2$ and $\left|V(D) \cap V_{t^{\prime}}\right| \geq 2$. Therefore, as $C$ and $D$ are given by Lemma 4.16, $C$ 2-intersects $V_{t}$ and $D$ 2-intersects $V_{t^{\prime}}$. As $G$ is 2-connected, we may assume that $V(C) \cap V_{t}=$ $V(D) \cap V_{t}=\{a, b\}$. Let $C^{\prime}$ and $C^{\prime \prime}$ be the two $a b$-parts of $C$. Let $D^{\prime}$ and $D^{\prime \prime}$ be the two $a b$ parts of $D$. As $|V(C) \cap V(D)|=2$, we can conclude that $\left|C^{\prime}\right|=\left|C^{\prime \prime}\right|=\left|D^{\prime}\right|=\left|D^{\prime \prime}\right|$. We may assume that $w \notin V\left(D^{\prime}\right)$. Hence, $C^{\prime} \cdot D^{\prime}$ is a longest cycle that 2-crosses $V_{t}$ at $\{x, y\}=V(C) \cap V_{t}$, a contradiction to the fact that $C$ is a 2-attractor for $V_{t}$. 


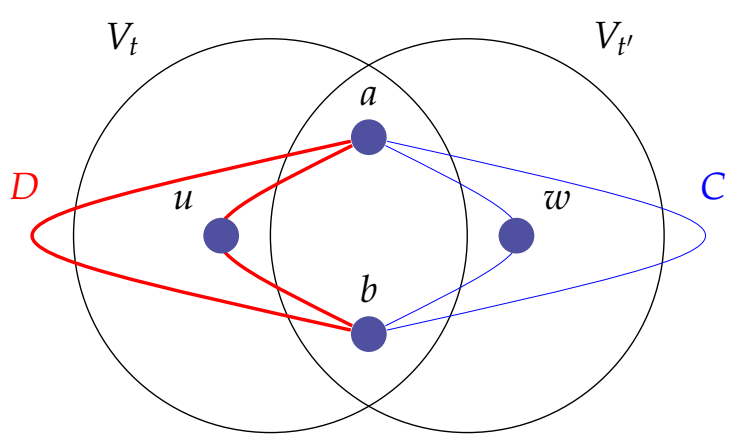

Figure 4.8: Situation in the proof of Theorem 4.18 .

The previous theorem implies the following result.

Corollary 4.19. All longest cycles intersect in 2-connected series-parallel graphs.

Again, as in the case of longest paths (Corollary 3.11), we have the following corollary due to results of Fomin and Thilikos [FT06], and Alon, Seymour, and Thomas [AST90]. Corollary 4.20. For every 2-connected planar graph $G$ on $n$ vertices, we have $\operatorname{lct}(G)<3.182 \sqrt{n}$, and for every 2-connected $K_{r}$-minor free graph $G$, we have $\operatorname{lct}(G)<r^{1.5} \sqrt{n}$.

\subsection{Partial 3-Trees}

In this section we focus on graphs with treewidth at most 3, also known as partial 3-trees (Proposition 2.2. By Theorem 4.18, every 2-connected partial 3-tree has a longest cycle transversal of cardinality at most two. We show that in fact there is a transversal of cardinality one, that is, all longest cycles intersect in a common vertex in 2-connected partial 3-trees (Theorem 4.22.

The proof is longer than the previous proofs on longest cycles, so we break the presentation in four subsections. In the first one, we state the main lemma (Lemma 4.21) and proceed to the proof of the main result (Theorem 4.22). The other three subsections contain the most technical parts of the proof. The second subsection introduces new definitions to be used along all the proofs. The third subsection contains the proof of the main lemma, using auxiliary results proved in the fourth subsection.

\subsubsection{Proof of the main theorem}

Generally speaking, the main idea of the proof is maintained (Section 2.5). The next lemma conceals the heart of the proof of Theorem 4.22 . The proof of that lemma is presented in Subsection 4.4.3.

Lemma 4.21. Let $G$ be a 2-connected graph with treewidth 3. Let $(T, \mathcal{V})$ be a full tree decomposition of $G$ and $V_{t} \in \mathcal{V}$. Then either $\operatorname{lct}(G)=1$ or there exists a longest cycle in $G$ that is fenced by $V_{t}$ and intersects $V_{t}$ at most three times. 
Using this lemma, we derive the main result of this section.

Theorem 4.22. If $G$ is a 2-connected partial 3-tree, then $\operatorname{lct}(G)=1$.

Proof. If $\operatorname{tw}(G) \leq 2$ then $\operatorname{lct}(G)=1$ by Theorem 4.18 . Therefore, we may assume that $\operatorname{tw}(G)=3$. Assume by contradiction that $\operatorname{lct}(G)>1$. By Proposition 2.18, $L:=L(G) \geq 5$. Let $(T, \mathcal{V})$ be a full tree decomposition of $G$, which exists by Proposition 2.1. For every $t \in V(T)$, let $\mathcal{F}(t)$ be the set of longest cycles in $G$ that are fenced by $V_{t}$ and intersect $V_{t}$ at most three times. By Lemma $4.21, \mathcal{F}(t) \neq \varnothing$ for every $t \in V(T)$. Observe that, as $L \geq 5$, for every such $t$, no cycle in $\mathcal{F}(t)$ is contained in $G\left[V_{t}\right]$. Thus, by Lemma 2.14, there exists an edge $t t^{\prime} \in E(T)$ and two longest cycles $C \in \mathcal{F}(t)$ and $D \in \mathcal{F}\left(t^{\prime}\right)$ such that $\operatorname{Branch}_{t}(C)=\operatorname{Branch}_{t}\left(t^{\prime}\right)$ and $\operatorname{Branch}_{t^{\prime}}(D)=\operatorname{Branch}_{t^{\prime}}(t)$.

Note that the bags containing vertices of $C$ are in $\operatorname{Branch}_{t}\left(t^{\prime}\right) \cup\{t\}$, and the bags containing vertices of $D$ are in $\operatorname{Branch}_{t^{\prime}}(t) \cup\left\{t^{\prime}\right\}$. Since Branch ${ }_{t}\left(t^{\prime}\right)$ and Branch $_{t^{\prime}}(t)$ are disjoint, $V(C) \cap V(D) \subseteq V_{t} \cup V_{t^{\prime}}$. Let $V_{t}=\{a, b, c, u\}$ and $V_{t^{\prime}}=\{a, b, c, w\}$.

Suppose for a moment that $u \in V(C)$. As $C$ is not contained in $G\left[V_{t}\right]$, and $\operatorname{Branch}_{t}(C)=\operatorname{Branch}_{t}\left(t^{\prime}\right), C$ has a vertex in $\operatorname{Branch}_{t}\left(t^{\prime}\right)$. So $C$ 3-intersects $V_{t}$ by Proposition 4.17, Let $V(C) \cap V_{t}=\{u, a, b\}$. Then $u a, u b \in E(C)$. Let $C_{a u}, C_{b u}, C_{a b}$ be the respective $a b u$-parts of $C$. As $\left|C_{a u}\right|=\left|C_{b u}\right|=1$ and $L \geq 5$, we conclude that $\left|C_{a b}\right| \geq 3$. Suppose for a moment that $w \in V(D)$. By a similar analysis, with $D$ instead of $C$, we have that $\left|V(D) \cap V_{t^{\prime}}\right|=3$. If $V(D) \cap V_{t^{\prime}}=\{w, a, b\}$, then $a w, b w \in E(D)$, so $(D-a w-b w) \cdot C_{a b}$ is a cycle and, as $\left|C_{a b}\right| \geq 3$, this is a contradiction. Hence, without loss of generality, $V(D) \cap V_{t^{\prime}}=\{w, b, c\}$, which implies that $b w, c w \in E(D)$. As $|V(C) \cap V(D)| \geq 2$, either $u \in V(D)$ or $w \in V(C)$. Without loss of generality, suppose that $u \in V(D)$. If $w \notin V(C)$, then $(C-u b) \cdot(D-u b)$ is a cycle longer than $L$, a contradiction (Figure 4.9(a)). If $w \in V(C)$, then $(C-u b-b w) \cdot(D-u b-b w)$ is a cycle longer than $L$, again a contradiction (Figure 4.9 (b)). Hence, $w \notin V(D)$.

As $|V(C) \cap V(D)| \geq 2$, at least two of $\{a, b, u\}$ are in $D$. Suppose for a moment that both $a$ and $b$ are in $D$. Let $D^{\prime}$ and $D^{\prime \prime}$ be the two $a b$-parts of $D$, with $\left|D^{\prime}\right| \geq\left|D^{\prime \prime}\right|$. If $u \notin V\left(D^{\prime}\right)$, then $(C-u a-u b) \cdot D^{\prime}$ is a cycle longer than $L$, a contradiction (Figure 4.9 (c)). So, $u \in V\left(D^{\prime}\right)$. Let $D_{a}$ and $D_{b}$ be the corresponding $u$-tails of $D^{\prime}$. As $\left|D^{\prime}\right| \geq 3$, we may assume without loss of generality that $\left|D_{a}\right| \geq 2$. But then $(C-u a) \cdot D_{a}$ is a cycle longer than $L$, a contradiction. Therefore, without loss of generality, we may assume that $a, u \in V(D)$. Let $D^{\prime}$ and $D^{\prime \prime}$ be the two au-parts of $D$, with $\left|D^{\prime}\right| \geq\left|D^{\prime \prime}\right|$. As $\left|D^{\prime}\right| \geq 3,(C-a u) \cdot D^{\prime}$ is a cycle longer than $L$, again a contradiction. We conclude that $u \notin V(C)$.

Analogously, we can conclude that $w \notin V(D)$ and therefore $V(C) \cap V(D) \subseteq V_{t} \cap V_{t^{\prime}}$. As $|V(C) \cap V(D)| \geq 2$, this implies that $\left|V(C) \cap V_{t}\right| \geq 2$ and $\left|V(D) \cap V_{t^{\prime}}\right| \geq 2$. Observe 


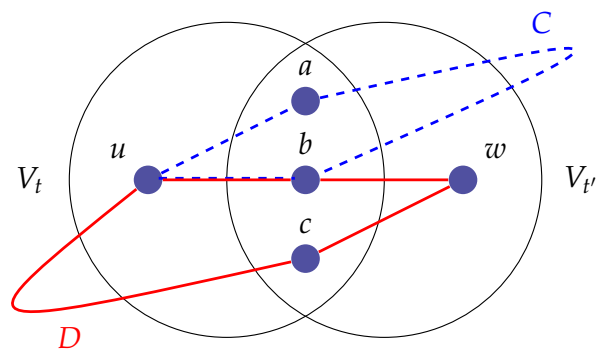

(a)

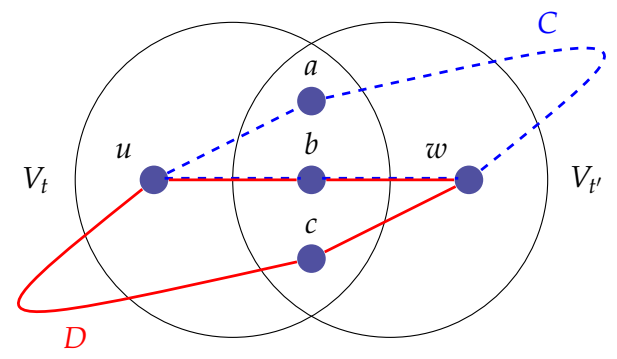

(b)

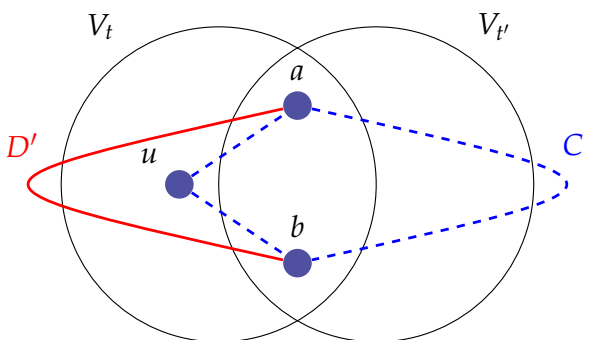

(c)

Figure 4.9: Cycles $C$ and $D$ as in the proof of Theorem 4.22. (a) $w \in V(D)$ and $w \notin V(C)$. (b) $w \in V(D)$ and $w \in V(C)$. (c) $w \notin V(D)$.

that any other longest cycle intersects $V_{t} \cap V_{t^{\prime}}$ at least twice. Otherwise it would intersect $C$ or $D$ at most once, a contradiction. As $\operatorname{lct}(G)>1$, there exists a longest cycle $F$ that does not contain $a$ and a longest cycle $H$ that does not contain $c$. By the previous observation, $F$ intersects $V_{t} \cap V_{t^{\prime}}$ at $\{b, c\}$ and $H$ intersects $V_{t} \cap V_{t^{\prime}}$ at $\{a, b\}$. Let $F^{\prime}$ and $F^{\prime \prime}$ be the two $b c$-parts of $F$, with $\left|F^{\prime}\right| \geq\left|F^{\prime \prime}\right|$. Let $H^{\prime}$ and $H^{\prime \prime}$ be the two $a b$ parts of $H$, with $\left|H^{\prime}\right| \geq\left|H^{\prime \prime}\right|$. The rest of the proof is divided into whether $C$ and $D$ 2-intersect or 3-intersect $V_{t} \cap V_{t^{\prime}}$.

Case 1: $C$ 2-intersects $V_{t} \cap V_{t^{\prime}}$.

Without loss of generality, assume that $C$ 2-intersects $V_{t} \cap V_{t^{\prime}}$ at $\{a, b\}$. Suppose for a moment that $F^{\prime}$ is internally disjoint from $C$. As $F$ and $C$ intersect each other at least twice, $F^{\prime \prime}$ and $C$ intersect each other at a vertex $x$ different from $b$. Let $F_{c x}^{\prime \prime}$ be the subpath of $F^{\prime \prime}$ that is internally disjoint from $C$, starts at $c$ and ends at $x$. Let $C_{x b}$ be the subpath of $C$ with extremes $x$ and $b$ such that $\left|C_{b x}\right| \geq L / 2$. Then $F^{\prime} \cdot F_{c x}^{\prime \prime} \cdot C_{x b}$ is a cycle longer than $L$, a contradiction (Figure 4.10(a)). We conclude that $F^{\prime}$ is internally disjoint from $D$. If $D$ 2-intersects $V_{t} \cap V_{t^{\prime}}$, then, as $|V(C) \cap V(D)| \geq 2$, we must have that $D$ intersects $V_{t} \cap V_{t^{\prime}}$ at $\{a, b\}$. But then we obtain a contradiction as before, with $D$ instead of $C$. So $D$ 3-intersects $V_{t} \cap V_{t^{\prime}}($ at $\{a, b, c\})$. Let $D_{a b}, D_{a c}, D_{b c}$ be the $a b c$-parts of $D$. As $C^{\prime \prime} \cdot D_{a b}$ and $\left(D-D_{a b}\right) \cdot C^{\prime}$ are both longest cycles, we have that $\left|C^{\prime}\right|=\left|D_{a b}\right|$. Similarly, $\left|C^{\prime \prime}\right|=\left|D_{a b}\right|$. Hence $\left|C^{\prime}\right|=\left|C^{\prime \prime}\right|=\left|D_{a b}\right|=L / 2$. As $F^{\prime}$ is internally disjoint from $D, D_{a b} \cdot F^{\prime} \cdot D_{c a}$ is a cycle longer than $L$, again a contradiction (Figure 4.10(b)). 
Case 2: Both $C$ and $D$ 3-intersect $V_{t} \cap V_{t^{\prime}}$.

Let $C_{a b}, C_{a c}, C_{b c}$ be the $a b c$-parts of $C$. Let $D_{a b}, D_{a c}, D_{b c}$ be the $a b c$-parts of $D$. Assume without loss of generality that $F^{\prime}$ is internally disjoint from $C$. Observe that both $(C-$ $\left.C_{b c}\right) \cdot F^{\prime}$ and $C_{b c} \cdot F^{\prime}$ are cycles. We conclude that $\left|C_{b c}\right|=\left|C_{a b}\right|+\left|C_{a c}\right|=\left|F^{\prime}\right|=\left|F^{\prime \prime}\right|=$ $L / 2$. As $\left(C-C_{b c}\right) \cdot D_{b c}$ and $\left(D-D_{b c}\right) \cdot C_{b c}$ are cycles, $\left|C_{b c}\right|=\left|D_{b c}\right|=L / 2$. A similar analysis with $H$ instead of $F$ shows us that $\left|C_{a b}\right|=\left|D_{a b}\right|=L / 2$. This implies that $\left|C_{a c}\right|=0$, a contradiction (Figure 4.10 (c)).

This concludes the proof of the theorem.

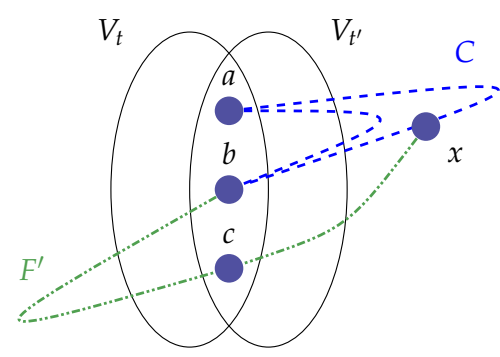

(a)

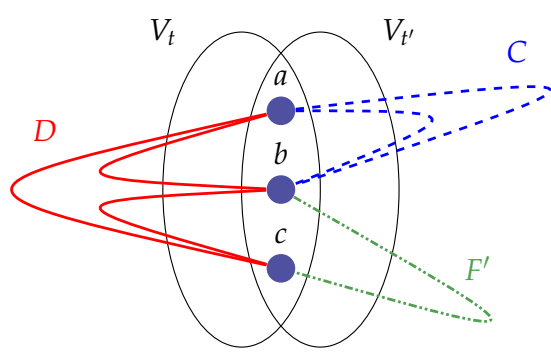

(b)

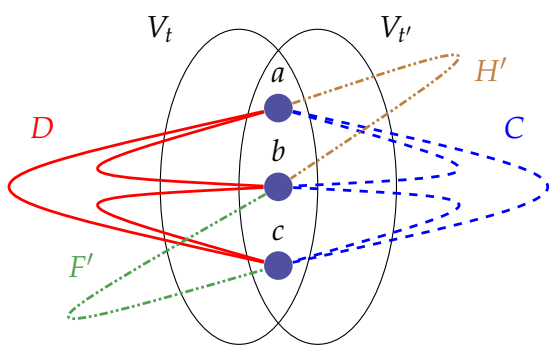

(c)

Figure 4.10: Cycles $C, D, F$ and $H$ as in the proof of Theorem 4.22. (a) $C$ 2-intersects $V_{t} \cap V_{t^{\prime}}$ and $F^{\prime}$ is internally disjoint from $C$. (b) $C$ 2-intersects $V_{t} \cap V_{t^{\prime}}$ and $F^{\prime}$ is internally disjoint from $D$. (c) C 3-intersects $V_{t} \cap V_{t^{\prime}}$.

\subsubsection{New definitions}

In what follows, we fix a 2-connected partial 3-tree $G$ such that $\operatorname{tw}(G)=3$, and a full tree decomposition $(T, \mathcal{V})$ of $G$.

For every $t \in V(T)$, and for every triple of vertices $\Delta$ in $V_{t}$, it is denoted by $\mathscr{B}_{t}(\Delta)$ the union of the branches of the neighbors of $t$ in $T$ such that the corresponding bag contains $\Delta$. That is,

$$
\mathscr{B}_{t}(\Delta)=\bigcup\left\{\operatorname{Branch}_{t}\left(t^{\prime}\right): t t^{\prime} \in E(T) \text { and } \Delta \subseteq V_{t^{\prime}}\right\}
$$

For a vertex $v \in V(G)$, we say that $v$ is t-inside $\Delta$ if $v \in V_{t^{\prime}}$ for some $t^{\prime} \in V(T)$ with $\operatorname{Branch}_{t}\left(t^{\prime}\right) \subseteq \mathscr{B}_{t}(\Delta)$. Otherwise, we say that $v$ is $t$-outside $\Delta$. When the context is 
clear, we just say that $v$ is inside or outside $\Delta$.

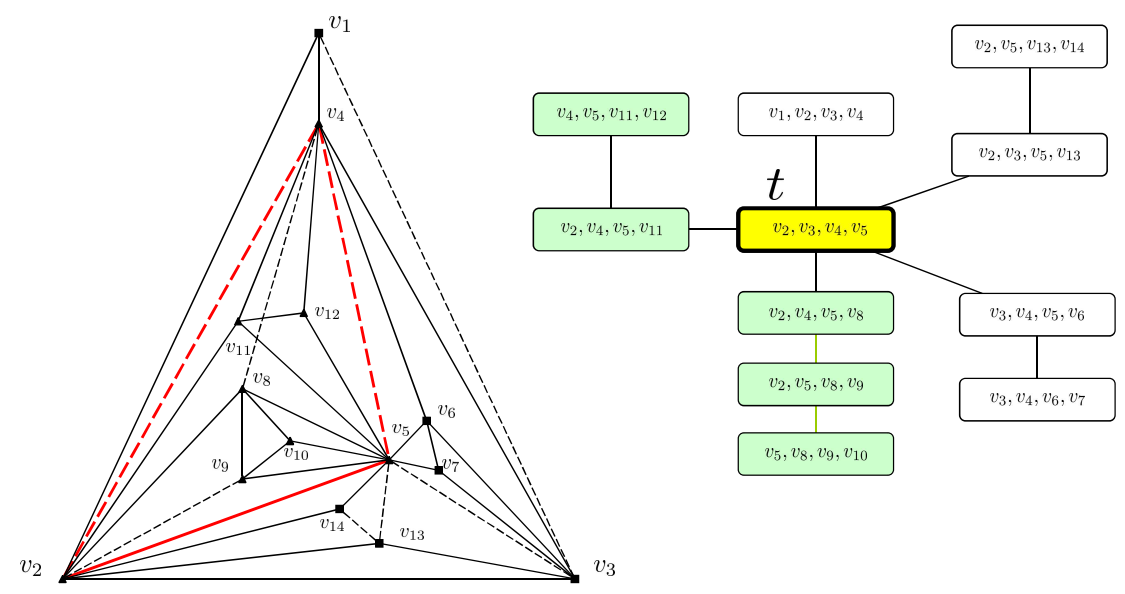

Figure 4.11: A partial 3-tree of treewidth 3 and a corresponding full tree decomposition. The dashed lines imply that there is no edge between the corresponding vertices. If $t$ is the node with $V_{t}=\left\{v_{2}, v_{3}, v_{4}, v_{5}\right\}$ and $\Delta=\left\{v_{2}, v_{4}, v_{5}\right\}$, then the vertices $v_{2}, v_{4}, v_{5}, v_{8}, v_{9}, v_{10}, v_{11}, v_{12}$ are $t$ inside $\Delta$ and all the other vertices are $t$-outside $\Delta$. Also, the cycles $v_{2} v_{5} v_{8} v_{2}$ and $v_{4} v_{12} v_{5} v_{11} v_{4}$ are $t$-inside $\Delta$, the cycle $v_{5} v_{7} v_{3} v_{4} v_{6} v_{5}$ is $t$-outside $\Delta$, and the cycles $v_{2} v_{5} v_{14} v_{2}$ and $v_{3} v_{4} v_{11} v_{5} v_{6} v_{3} t$ jump $\Delta$.

Given a path $C^{\prime}$ that 2-intersects $\Delta$ at the extremes of $C^{\prime}$, we say that $C^{\prime}$ is $t$-inside $\Delta$ if every vertex of $C^{\prime}$ is $t$-inside $\Delta$, and $C^{\prime}$ is t-outside $\Delta$ otherwise. Given a cycle $C$ that intersects $\Delta$ at least twice, we say that $C$ is $t$-inside $\Delta$ if every $\Delta$-part of $C$ is $t$ inside $\Delta, C$ is t-outside $\Delta$ if every $\Delta$-part of $C$ is t-outside $\Delta$, and $C$ t-jumps $\Delta$ if $C$ has a $\Delta$-part $t$-inside $\Delta$ and a $\Delta$-part $t$-outside $\Delta$.

Again, if the context is clear, we just say that $C$ is inside, outside, or jumps $\Delta$ (Figure 4.11. Furthermore, we abuse notation and, when the context is clear, for a cycle $C$ that jumps $\Delta$, we say that $C \ell$-jumps $\Delta$ if $|V(C) \cap \Delta|=\ell$. Observe that $\ell \in\{2,3\}$. Moreover, if $C$-jumps $\Delta$, we say that $C$-jumps $\Delta$ at $V(C) \cap \Delta$. Throughout this chapter, we abreviate a triple of vertices $\{a, b, c\}$ by $a b c$.

Proposition 4.23. Let $G$ be a graph with $\operatorname{tw}(G)=3$ and let $(T, \mathcal{V})$ be a full tree decomposition of $G$. Let $t \in V(T)$ and $V_{t}=\{a, b, c, d\}$. If $C^{\prime}$ is a path with extremes $a$ and $b$ that 2intersects $V_{t}$ at $\{a, b\}$, then $\operatorname{Branch}_{t}\left(C^{\prime}\right) \subseteq \mathscr{B}_{t}(a b c) \cup \mathscr{B}_{t}(a b d)$.

Proof. If $V\left(C^{\prime}\right) \subseteq V_{t}$ then, by definition, $\operatorname{Branch}_{t}\left(C^{\prime}\right)=\varnothing$ and we are done. Thus, by Proposition 2.13, there exists an edge $t t^{\prime} \in E(T)$ such that $\operatorname{Branch}_{t}\left(C^{\prime}\right)=\operatorname{Branch}_{t}\left(t^{\prime}\right)$. As $\left|V\left(C^{\prime}\right) \cap V_{t}\right|=2$ and $V\left(C^{\prime}\right) \nsubseteq V_{t}, \quad C^{\prime}$ does not have edges in $G\left[V_{t}\right]$. Hence, by Proposition 2.8, $\{a, b\}=V_{t} \cap C^{\prime} \subseteq V_{t^{\prime}}$. Since $\left|V_{t} \cap V_{t^{\prime}}\right|=3$, we have that either $V_{t} \cap V_{t^{\prime}}=\{a, b, c\}$ or $V_{t} \cap V_{t^{\prime}}=\{a, b, d\}$. Hence $\operatorname{Branch}_{t}\left(C^{\prime}\right)=\operatorname{Branch}_{t}\left(t^{\prime}\right) \subseteq \mathscr{B}_{t}(a b c) \cup \mathscr{B}_{t}(a b d)$. 


\subsubsection{Proof of the main lemma}

For all lemmas in this subsection, we fix a graph $G$ with $\operatorname{tw}(G)=3$ and $\operatorname{lct}(G)>1$, a full tree decomposition $(T, \mathcal{V})$ of $G$, and a node $t \in V(T)$. We let $\mathcal{X}_{2}$ be the set of all longest cycles in $G$ that 2-cross $V_{t}$, and let $\mathcal{F}$ be the set of all longest cycles in $G$ that are fenced by $V_{t}$ and intersect $V_{t}$ at most three times. Therefore, proving Lemma 4.21 reduces to prove that $\mathcal{F} \neq \varnothing$.

Proof of Lemma 4.21. Suppose that $\mathcal{X}_{2} \neq \varnothing$. If there are two non $V_{t}$-equivalent longest cycles in $\mathcal{X}_{2}$, then we are done by Lemma 4.24. If all cycles in $\mathcal{X}_{2}$ are $V_{t}$-equivalent, then we are done by Lemma 4.25 . Hence $\mathcal{X}_{2}=\varnothing$, and we are done by Lemma 4.28 .

Lemma 4.24. If there are two non $V_{t}$-equivalent longest cycles in $\mathcal{X}_{2}$, then $\mathcal{F} \neq \varnothing$.

Proof. Let $V_{t}=\{a, b, c, d\}$. Suppose by contradiction that $C, D \in \mathcal{X}_{2}, V(C) \cap V_{t}=\{a, b\}$ and $V(D) \cap V_{t} \neq\{a, b\}$. Let $C^{\prime}$ and $C^{\prime \prime}$ be the corresponding ab-parts of $C$. By Proposition 4.23, we have that $\operatorname{Branch}_{t}\left(C^{\prime}\right), \operatorname{Branch}_{t}\left(C^{\prime \prime}\right) \subseteq \mathscr{B}_{t}(a b c) \cup \mathscr{B}_{t}(a b d)$. If $D$ 2-crosses $V_{t}$ at $\{c, d\}$, let $D^{\prime}$ and $D^{\prime \prime}$ be the corresponding $c d$-parts of $D$. By Proposition 4.23, we have that $\operatorname{Branch}_{t}\left(D^{\prime}\right), \operatorname{Branch}_{t}\left(D^{\prime \prime}\right) \subseteq \mathscr{B}_{t}(a c d) \cup \mathscr{B}_{t}(b c d)$, which implies that $V(C) \cap V(D)=\varnothing$, a contradiction. Hence, we may assume that $D$ 2-crosses $V_{t}$ at $\{a, c\}$. Let $D^{\prime}$ and $D^{\prime \prime}$ be the corresponding ac-parts of $D$. By Proposition 4.23, we have that $\operatorname{Branch}_{t}\left(D^{\prime}\right), \operatorname{Branch}_{t}\left(D^{\prime \prime}\right) \subseteq \mathscr{B}_{t}(a c d) \cup \mathscr{B}_{t}(a b c)$. Also, as both $C$ and $D$ cross $V_{t}$, we may assume, without loss of generality, that $C^{\prime}$ is internally disjoint from $D^{\prime}$ and that $C^{\prime \prime}$ is internally disjoint from $D^{\prime \prime}$. As $\operatorname{lct}(G)>1$, there exists a longest cycle $F$ that does not contain $a$. If $F$ is fenced by $V_{t}$, we are done, so let us assume that $F$ crosses $V_{t}$.

First consider the case in which $F$ 2-intersects $V_{t}$. If $F$ intersects $V_{t}$ at $\{b, d\}$ then, by Proposition 4.23, we have that $\operatorname{Branch}_{t}\left(F^{\prime}\right), \operatorname{Branch}_{t}\left(F^{\prime \prime}\right) \subseteq \mathscr{B}_{t}(a b d) \cup \mathscr{B}_{t}(b c d)$, where $F^{\prime}$ and $F^{\prime \prime}$ are the corresponding $b d$-parts of $F$. Hence, $D$ and $F$ do not intersect each other, a contradiction. By a similar argument, with $C$ instead of $D$, we cannot have that $F$ intersects $V_{t}$ at $\{c, d\}$. Thus, $F$ 2-intersects $V_{t}$ at $\{b, c\}$. Let $F^{\prime}$ and $F^{\prime \prime}$ be the corresponding $b c$-parts of F. By Proposition 4.23. $\operatorname{Branch}_{t}\left(F^{\prime}\right), \operatorname{Branch}_{t}\left(F^{\prime \prime}\right) \subseteq \mathscr{B}_{t}(a b c) \cup \mathscr{B}_{t}(b c d)$. Suppose that $C, D$ and $F$ are $t$-inside $a b c$. As $G$ is 2-connected, there exist two internally disjoint paths starting at $d$ and ending at distinct vertices in $\{a, b, c\}$. Let $P$ and $Q$ be these two paths, and suppose without loss of generality that $b$ is an extreme of $P$ and that $c$ is an extreme of $Q$. By Proposition 2.7. $a b c$ separates $d$ from any vertex inside $a b c$ different from any of $\{a, b, c\}$, so both $P$ and $Q$ are internally disjoint from $C$ and $D$. But then $P \cdot Q \cdot D^{\prime} \cdot C^{\prime}$ and $P \cdot Q \cdot D^{\prime \prime} \cdot C^{\prime \prime}$ are both cycles, at least one of them longer than $L$, a contradiction (Figure 4.12(a)). We conclude that at least one of $\{C, D, F\}$ is not $t$-inside $a b c$. Without loss of generality, assume that $C$ is not $t$-inside $a b c$, and that 
$\operatorname{Branch}_{t}\left(C^{\prime \prime}\right) \nsubseteq \mathscr{B}_{t}(a b c)$. Hence $\operatorname{Branch}_{t}\left(C^{\prime \prime}\right) \subseteq \mathscr{B}_{t}(a b d)$. Thus $C^{\prime \prime}$ is internally disjoint from both $D$ and $F$. As $D$ and $F$ 2-cross $V_{t}$, we may assume that $D^{\prime}$ is internally disjoint from $F^{\prime}$ and that $D^{\prime \prime}$ is internally disjoint from $F^{\prime \prime}$. But then $C^{\prime \prime} \cdot D^{\prime} \cdot F^{\prime}$ and $C^{\prime \prime} \cdot D^{\prime \prime} \cdot F^{\prime \prime}$ are both cycles, at least one of them longer than $L$, a contradiction (Figure $4.12(\mathrm{~b})$ ).

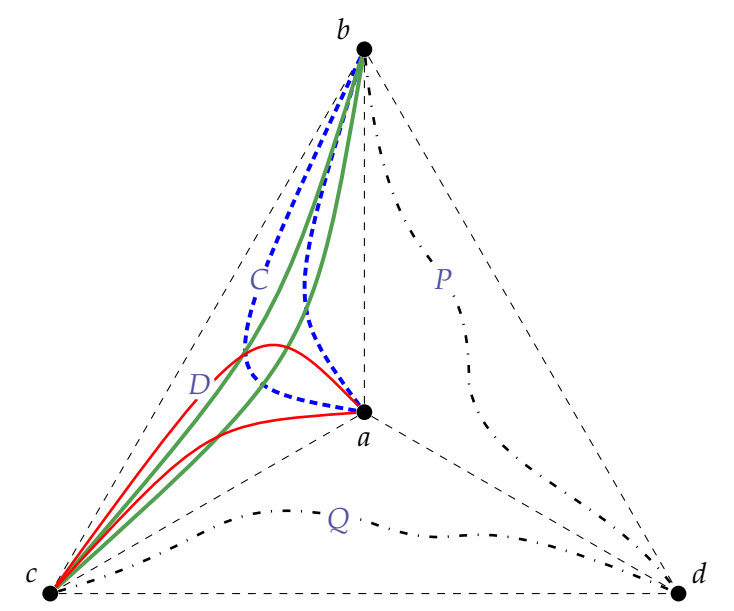

(a)

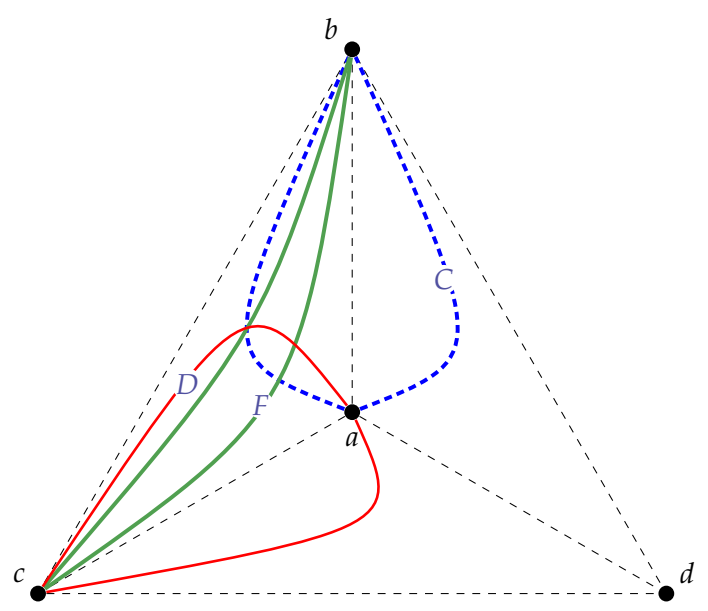

(b)

Figure 4.12: (a) $C, D$ and $F$ are $t$-inside $a b c$. (b) At least one of $\{C, D, F\}$ is not $t$-inside $a b c$.

Now suppose that $F$ 3-intersects $V_{t}($ at $\{b, c, d\})$. Let $F_{b c}, F_{c d}$ and $F_{b d}$ be the respective $b c d$-parts of $F$. If both $C$ and $D$ are $t$-inside $a b c$, then we can proceed as in the previous paragraph with $F_{b d}$ and $F_{c d}$ instead of $P$ and $Q$ respectively. If both $C$ and $D$ are $t$-outside $a b c$, then $\operatorname{Branch}_{t}\left(C^{\prime}\right), \operatorname{Branch}_{t}\left(C^{\prime \prime}\right) \subseteq \mathscr{B}_{t}(a b d)$ and $\operatorname{Branch}_{t}\left(D^{\prime}\right), \operatorname{Branch}_{t}\left(D^{\prime \prime}\right) \subseteq \mathscr{B}_{t}(a c d)$, which implies that $V(C) \cap V(D)=\{a\}$, a contradiction. Hence, at least one of $\{C, D\} t$-jumps $a b c$. Without loss of generality, suppose that $C$ t-jumps $a b c$ and that $\operatorname{Branch}_{t}\left(C^{\prime \prime}\right) \subseteq \mathscr{B}_{t}(a b d)$, which implies that $C^{\prime \prime}$ is internally disjoint from $D$. By Proposition 4.23 , $\operatorname{Branch}_{t}\left(F_{b d}\right) \subseteq \mathscr{B}_{t}(a b d) \cup \mathscr{B}_{t}(b c d)$, hence $F_{b d}$ is internally disjoint from $C^{\prime}$ and $D$. By the same proposition, $F_{c d}$ is disjoint from $C$. Let $R$ be the subpath of $F_{b d}$ that starts at $d$, ends at a vertex of $C^{\prime \prime}$ and is internally disjoint from $C^{\prime \prime}$. Such a subpath must exist, because $C^{\prime \prime}$ does not contain $d$ and $F_{b d}$ contains $b$. Suppose that the other extreme of $R$ is $x$. First consider the case in which $D$ also $t$-jumps $a b c$, and without loss of generality suppose that $\operatorname{Branch}_{t}\left(D^{\prime}\right) \subseteq \mathscr{B}_{t}(a c d)$. Let $S$ be the subpath of $F_{c d}$ that starts at $d$, ends at $D^{\prime}$ and is internally disjoint from $D^{\prime}$. Such a subpath must exist, because $D^{\prime}$ does not contain $d$ and $F_{c d}$ contains $c$. Suppose that the other extreme of $R$ is $y$. Let $C_{a x}^{\prime \prime}$ and $C_{b x}^{\prime \prime}$ be the two $x$-tails of $C^{\prime \prime}$. Let $D_{a y}^{\prime}$ and $D_{c y}^{\prime}$ be the two $y$-tails of $D^{\prime}$. Then $C^{\prime} \cdot C_{b x}^{\prime \prime} \cdot R \cdot S \cdot D_{y a}^{\prime}$ and $D^{\prime \prime} \cdot D_{c y}^{\prime} \cdot S \cdot R \cdot C_{x a}^{\prime \prime}$ are cycles, at least one of them longer than $L$, a contradiction (Figure 4.13(a)). Finally consider the case in which $D$ is $t$-inside $a b c$. Recall that $C^{\prime}$ is internally disjoint from $D^{\prime}$. Then $C^{\prime} \cdot C_{b x}^{\prime \prime} \cdot R \cdot F_{d c} \cdot D^{\prime}$ and $D^{\prime \prime} \cdot C_{a x}^{\prime \prime} \cdot R \cdot F_{d c}$ are cycles, at least one of them longer 
than $L$, a contradiction (Figure $4.13(\mathrm{~b})$ ).

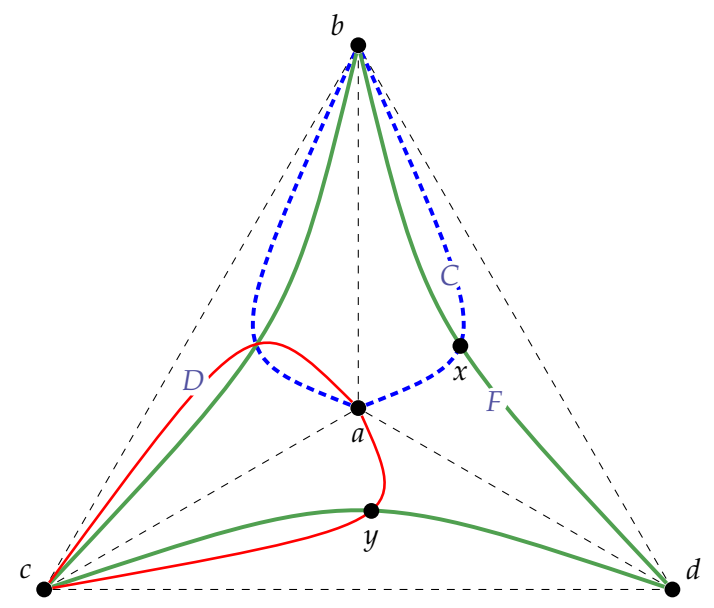

(a)

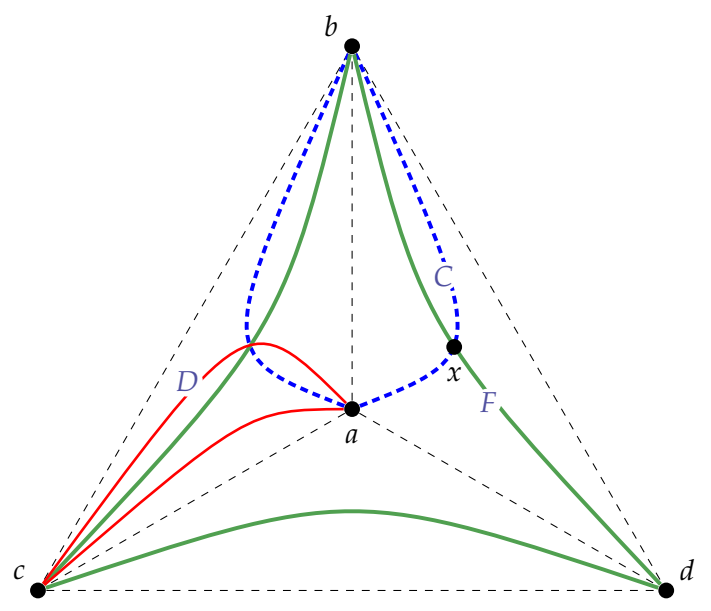

(b)

Figure 4.13: Cases in the proof of Lemma 4.24 F 3-intersects $V_{t}$. (a) $D$ t-jumps $a b c$. (b) $D$ is $t$-inside $a b c$.

Lemma 4.25. If $\mathcal{X}_{2} \neq \varnothing$ and all cycles in $\mathcal{X}_{2}$ are $V_{t}$-equivalent, then $\mathcal{F} \neq \varnothing$.

Proof. Let $V_{t}=\{a, b, c, d\}$. Let $C \in \mathcal{X}_{2}$. Assume without loss of generality that $V(C) \cap V_{t}=\{a, b\}$. As $\operatorname{lct}(G)>1$, there exists a longest cycle $D$ that does not contain $a$, and a longest cycle $F$ that does not contain $b$. If either $D$ or $F$ is fenced by $V_{t}, \mathcal{F} \neq \varnothing$. So, as all cycles in $\mathcal{X}_{2}$ are $V_{t}$-equivalent, both $D$ and $F$ 3-intersect $V_{t}$.

Claim 4.26. Every cycle that 2-crosses $V_{t}$ at $\{a, b\}$ jumps both $a b c$ and $a b d$.

Proof of Claim 4.26 . Let $\bar{C}$ be a longest cycle in $G$ that 2-crosses $V_{t}$ at $\{a, b\}$. Let $\bar{C}^{\prime}$ and $\bar{C}^{\prime \prime}$ be the two $a b$-parts of $\bar{C}$. Suppose by contradiction and, without loss of generality, that $\bar{C}$ is $t$-inside $a b c$, that is, $\operatorname{Branch}_{t}\left(\bar{C}^{\prime}\right), \operatorname{Branch}_{t}\left(\bar{C}^{\prime \prime}\right) \subseteq \mathscr{B}_{t}(a b c)$. Let $D^{\prime}$ and $D^{\prime \prime}$ be the two $b c$-parts of $D$. Let $F^{\prime}$ and $F^{\prime \prime}$ be the two $a c$-parts of $F$. As $D$ and $\bar{C}$ intersect in at least two vertices, we may assume that $D^{\prime}$ is $t$-inside $a b c$, and, moreover that $D^{\prime}$ internally intersects one of $\left\{\bar{C}^{\prime}, \bar{C}^{\prime \prime}\right\}$. Analogously, we may assume that $F^{\prime}$ is $t$-inside $a b c$, and, moreover that $F^{\prime}$ internally intersects one of $\left\{\bar{C}^{\prime}, \bar{C}^{\prime \prime}\right\}$. First suppose that both $D^{\prime}$ and $F^{\prime}$ internally intersect with the same part of $\bar{C}$, say $\bar{C}^{\prime}$. As $\bar{C}$ crosses $V_{t}, \bar{C}^{\prime \prime}$ is internally disjoint from both $D$ and $F$. Hence $D^{\prime \prime} \cdot \bar{C}^{\prime \prime} \cdot F^{\prime}$ and $F^{\prime \prime} \cdot \bar{C}^{\prime \prime} \cdot D^{\prime}$ are both cycles, one of them longer than $L$, a contradiction. Now suppose, without loss of generality, that $D^{\prime}$ internally intersects $\bar{C}^{\prime}$ and $F^{\prime}$ internally intersects $\bar{C}^{\prime \prime}$. Let $R$ be the subpath of $\bar{C}^{\prime}$ that starts at $a$, ends at a vertex $x$ of $D^{\prime}$ and is internally disjoint from $D^{\prime}$. Let $D_{c x}$ be the subpath of $D^{\prime}$ with extremes $c$ and $x$. Observe that, as $\bar{C}$ crosses $V_{t}, R$ is internally 
disjoint from $F$. Then $F^{\prime \prime} \cdot R \cdot D_{x c}$ and $\left(D-D_{c x}\right) \cdot R \cdot F^{\prime}$ are both cycles, at least one of them longer than $L$, again a contradiction.

Let $C^{\prime}$ and $C^{\prime \prime}$ be the two $a b$-parts of $C$. By Claim 4.26, we may assume that $C^{\prime}$ is $t$-inside $a b c$ and that $C^{\prime \prime}$ is $t$-inside $a b d$.

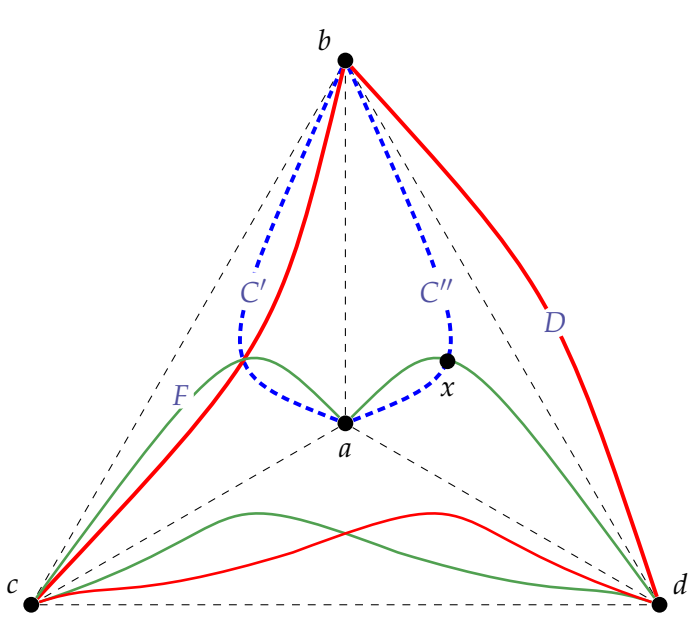

(a)

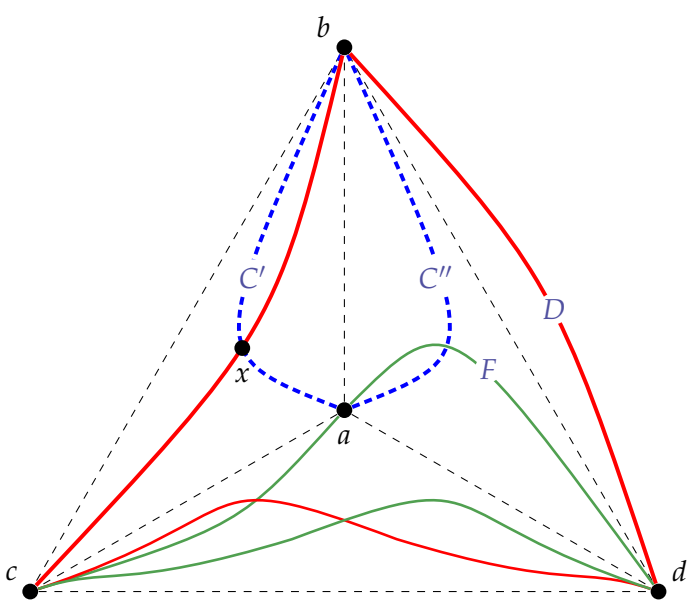

(c)

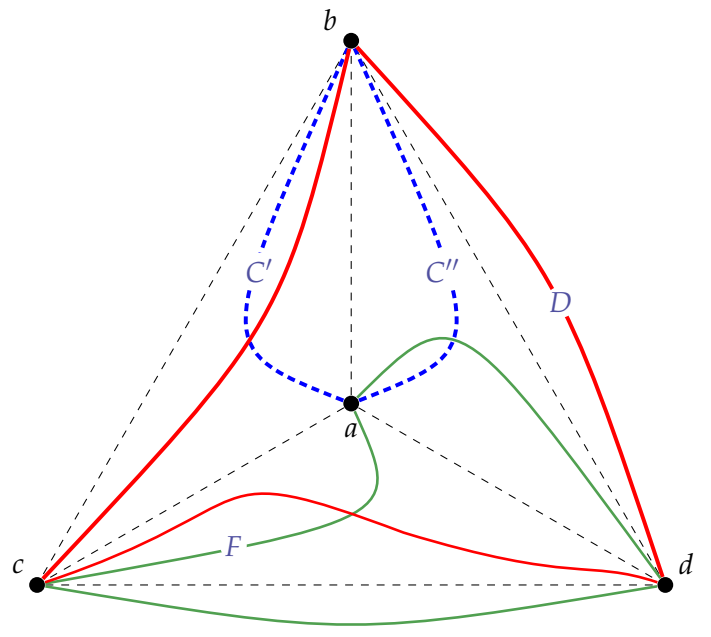

(b)

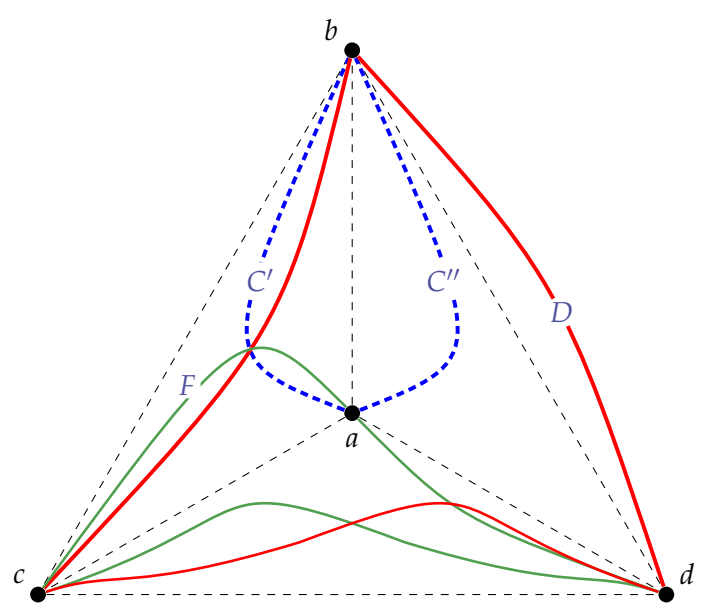

(d)

Figure 4.14: Cases in the proof of Claim 4.27 of Lemma 4.25 (a) $F$ jumps both $a b c$ and $a b d$. (b) $F$ jumps only $a b d$ and $F_{c d}$ does not jump acd. (c) $F$ jumps only $a b d$ and $F_{c d}$ jumps $a c d$. (d) $F$ jumps only $a b c$.

Claim 4.27. $D$ and F jump both $a b c$ and $a b d$.

Proof of Claim 4.27 If $D$ does not jump at least one of $\{a b c, a b d\}$, then $C$ and $D$ only intersect at $b$, a contradiction to the fact that $G$ is 2-connected. Hence $D$ jumps at least one of $\{a b c, a b d\}$. Analogously, $F$ jumps at least one of $\{a b c, a b d\}$. Suppose by contradiction that the claim is not true. Then, one of $\{D, F\}$, say $D$, jumps exactly one 
of $\{a b c, a b d\}$, say $a b c$. First suppose that $F$ jumps both of $\{a b c, a b d\}$. Recall that $C^{\prime \prime}$ is the $a b$-part of $C$ that is $t$-inside $a b d$. Let $R$ be the subpath of $C^{\prime \prime}$ that is internally disjoint from $F$, starts at $b$ and ends at a vertex $x$ of $F$ (possibly $x=a$ ). Let $F_{a c}, F_{c d}, F_{d x}$, and $F_{a x}$ be the corresponding $a c d x$-parts of $F$. Let $D_{b c}, D_{c d}$, and $D_{b d}$ be the corresponding $b c d$-parts of $D$. Then $F_{c d} \cdot F_{d x} \cdot R \cdot D_{b c}$ and $D_{c d} \cdot D_{d b} \cdot R \cdot F_{x a} \cdot F_{a c}$ are cycles, one of them longer than $L$, a contradiction (Figure $4.14(\mathrm{a})$ ).

So, $F$ jumps exactly one of $\{a b c, a b d\}$. Suppose for a moment that it is $a b d$. Let $F_{a c}, F_{c d}$, and $F_{a d}$ be the corresponding acd-parts of $F$. Let $D_{b c}, D_{c d}$, and $D_{b d}$ be the corresponding $b c d$-parts of $D$. If $D_{c d}$ is internally disjoint from $F_{a c}$, we proceed as before. Hence, we may assume that $D_{c d}$ is $t$-inside $a c d$. If $F_{c d}$ is $t$-outside $a c d$, then $F_{c d} \cdot D_{c d}$ and $D_{b c} \cdot F_{c a} \cdot F_{a d} \cdot D_{d b}$ are longest cycles, with the former with empty intersection with $C$, a contradiction (Figure $4.14(b)$ ). Thus, $F_{c d}$ is $t$-inside acd. Let $R$ be the subpath of $C^{\prime}$ that is internally disjoint from $D$, starts at $a$ and ends at a vertex $x$ of $D$ (possibly $x=b$ ). Let $D_{b d}, D_{d c}, D_{c x}$, and $D_{x b}$ be the corresponding $b d c x$-parts of $D$. Then $F_{d c} \cdot F_{c a} \cdot R \cdot D_{x b} \cdot D_{b d}$ and $D_{d c} \cdot D_{c x} \cdot R \cdot F_{a d}$ are cycles, one of them longer than $L$, a contradiction (Figure 4.14(c)). Now suppose that $F$ jumps $a b c$ (and not $a b d$ ). Then $F_{c d} \cdot F_{d a} \cdot C^{\prime \prime} \cdot D_{b c}$ and $D_{c d} \cdot D_{d b} \cdot C^{\prime \prime} \cdot F_{a c}$ are cycles, one of them longer than $L$, again a contradiction (Figure $4.14(\mathrm{~d})$ ).

By Claim 4.27 and Proposition 4.23, $\operatorname{Branch}_{t}\left(F_{a c}\right), \operatorname{Branch}_{t}\left(D_{b c}\right) \subseteq \mathscr{B}_{t}(a b c)$ and $\operatorname{Branch}_{t}\left(F_{a d}\right), \operatorname{Branch}_{t}\left(D_{b d}\right) \subseteq \mathscr{B}_{t}(a b d)$ (Figure 4.15).

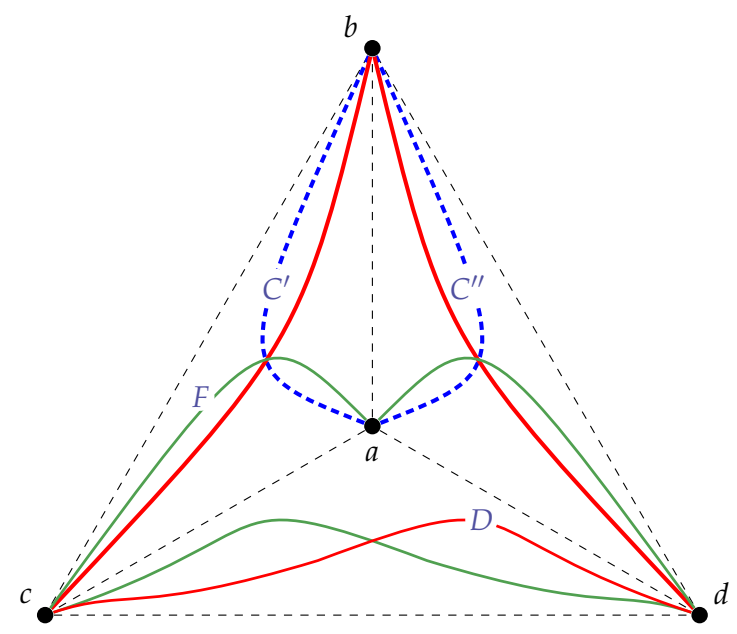

Figure 4.15: The structure given by Claim 4.27 of Lemma 4.25

Now, by Corollary 4.36 , as $\operatorname{lct}(G)>1$, there exists a longest cycle $H$ that does not $t$-jump abc. If $H$ intersects $V_{t}$ at most once, then $H$ is fenced by $V_{t}$ and $\mathcal{F} \neq \varnothing$. So $H$ intersects $V_{t}$ at least twice. Suppose for a moment that $H$ 2-intersects $V_{t}$. If $H$ is fenced by $V_{t}$, then $\mathcal{F} \neq \varnothing$ and we are done. Hence, $H \in \mathcal{X}_{2}$. As all cycles in $\mathcal{X}_{2}$ 
are equivalent, $H$ 2-intersects $V_{t}$ at $\{a, b\}$. By Claim 4.26, $H$ jumps $a b c$, a contradiction. So, $H$ intersects $V_{t}$ at least three times.

Suppose for a moment that $H$ 4-intersects $V_{t}$. As $H$ is a cycle, either $H$ has a subpath from $a$ to $c$ that is disjoint from $b$ and $d$, or $H$ has a subpath from $b$ to $c$ that is disjoint from $a$ and $d$. Without loss of generality assume the former. Let $H_{a c}$ be such subpath. Then there exists a subpath $R$ of $H_{a c}$ from $a$ to $D_{c d}$ that is internally disjoint from both $C$ and $D$. Let $x$ be its extreme in $D$. Let $D_{b c}, D_{c x}, D_{x d}$, and $D_{d b}$ be the $b c x d$-parts of $D$. Then $D_{b c} \cdot C^{\prime \prime} \cdot R \cdot D_{x c}$ and $C^{\prime} \cdot D_{b d} \cdot D_{d x} \cdot R$ are both cycles, one of them longer than $L$, a contradiction (Figure 4.16(a)). We conclude that $H$ 3-intersects $V_{t}$. If $H$ is $t$-inside $a b c$, then $H$ is fenced by $V_{t}$ by Corollary 4.36 , so $\mathcal{F} \neq \varnothing$ and we are done. Thus, $H$ is $t$-outside $a b c$.

If $H$ contains both $a$ and $c$, then $H_{a c}$, the subpath of $H$ from $a$ to $c$, is internally disjoint from $C$ and we proceed as before. Hence, either $H$ does not contain $a$ or $H$ does not contain $c$. Suppose for a moment that $H$ does not contain $a$. Then $H_{b c}$, the subpath of $H$ from $b$ to $c$, is internally disjoint from $C$ so we proceed as before (with $C$ and $F$ instead of $C$ and $D$ ). So, we may assume that $H$ does not contain $c$. Let $H_{a b}, H_{b d}$, and $H_{a d}$ be the $a b d$-parts of $H$. Let $F_{a c}, F_{c d}$, and $F_{a d}$ be the $a c d$-parts of $F$. As $H$ is $t$-outside $a b c$, we have that $H_{a b}$ is $t$-inside $a b d$. Moreover, observe that $H$ is $t$-inside $a b d$. Indeed, if for example $H_{b d}$ is $t$-outside $a b d$ then we proceed as before. The same reasoning applies if $H_{a d}$ is $t$-outside $a b d$. Thus, as $\left(F-F_{a d}\right) \cdot H_{a d}$ and $\left(F-F_{a d}\right) \cdot H_{a b} \cdot H_{b d}$ are cycles, we have that $\left|F_{a d}\right| \geq L / 2$. Now, repeating the argument with $a b d$ instead of $a b c$, we deduce that there exists a longest cycle $J$ that 3-intersects $V_{t}$ at $a b c$ and is t-inside $a b c$. Hence $\left|F_{a c}\right| \geq L / 2$. This implies that $\left|F_{c d}\right|=0$, a contradiction (Figure 4.16(b)).

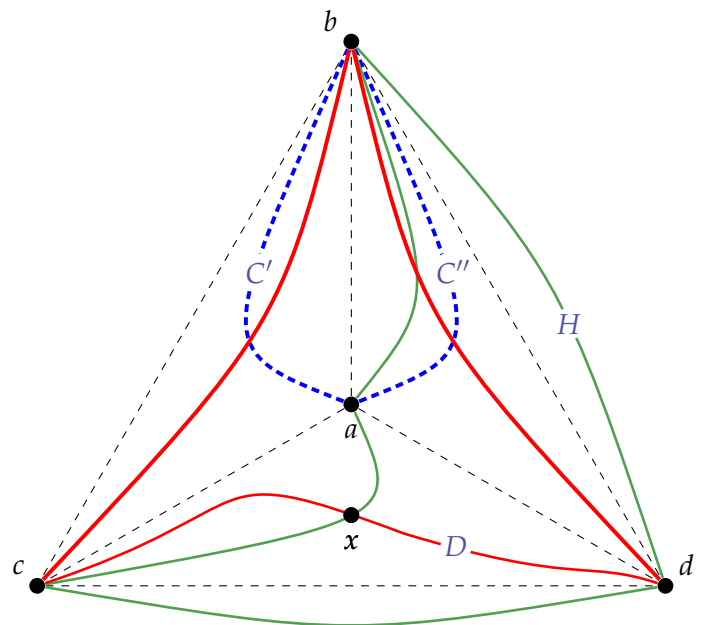

(a)

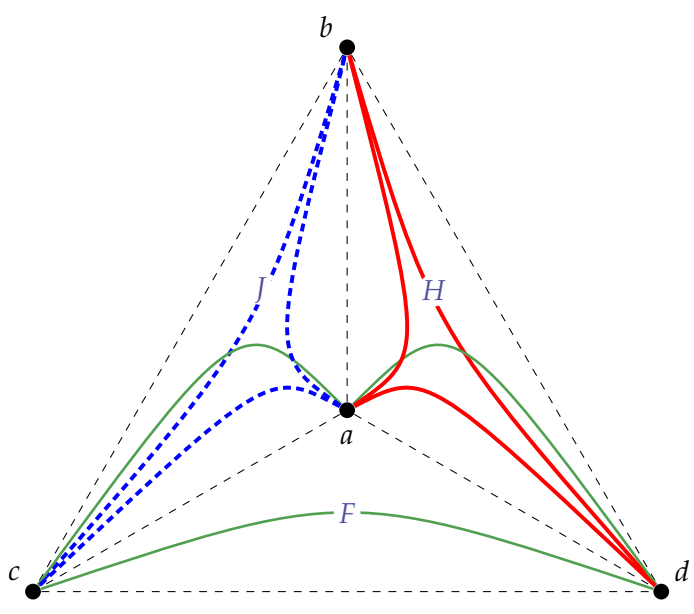

(b)

Figure 4.16: Cases in the last part of the proof of Lemma 4.25. (a) $H$ 4-intersects $V_{t}$. (b) $H$ 3-intersects $V_{t}$. 
The next lemma is the most technical one. Its proof is organized into four claims, namely, Claims 4.29, 4.30, 4.31, and 4.32. The proof of Claim 4.29 depends on an auxiliary result (Corollary 4.36) presented and proved ahead, in Subsection 4.4.4. Claim 4.30 depends on Claim 4.29, while Claims 4.31 and 4.32 depend on Claim 4.30 . The proof of Lemma 4.28 itself uses Claims $4.29,4.31$, and 4.32 .

Lemma 4.28. If $\mathcal{X}_{2}=\varnothing$, then $\mathcal{F} \neq \varnothing$.

Proof. As $\operatorname{lct}(G)>1$ and $\mathcal{X}_{2}=\varnothing$, for every triple of vertices $\Delta \subseteq V_{t}$, there exists a nonempty collection $\mathscr{C}_{\Delta}$ of longest cycles that 3-intersect $V_{t}$ at $\Delta$. Suppose by contradiction that $\mathcal{F}=\varnothing$. Then, for every $\Delta \subseteq V_{t}$, every cycle in $\mathscr{C}_{\Delta}$ crosses $V_{t}$. Let $\mathscr{C}=\left\{\mathscr{C}_{\Delta}: \Delta \subseteq V_{t}\right\}$.

Claim 4.29. For every triple of vertices $\Delta \subseteq V_{t}$, at least one cycle in $\mathscr{C} \backslash \mathscr{C}_{\Delta}$ does not jump $\Delta$.

Proof of Claim 4.29. Suppose by contradiction that all cycles in $\mathscr{C} \backslash \mathscr{C}_{a b c}$ jump abc. (The other cases are analogous.) Then, as $\mathscr{C}_{a b d}, \mathscr{C}_{b c d}, \mathscr{C}_{a c d} \neq \varnothing$, for every $\{i, j\} \subseteq a b c$, there exists a longest cycle that 2-jumps $a b c$ at $\{i, j\}$. Hence, by Corollary 4.36 applied to $a b c$, there exists a longest cycle $C$ that does not jump $a b c$. If $C$ intersects $V_{t}$ at most once or is as item (ii) or (iii) of Corollary 4.36 , then, as $\mathcal{X}_{2}=\varnothing, C$ is fenced by $a b c$, which implies that $\mathcal{F} \neq \varnothing$, a contradiction. Thus, we may assume that $C$ is as item $(i)$ of Corollary 4.36, that is, $C$ is outside $a b c$.

Case 1: $C$ 3-intersects $V_{t}$.

As all cycles in $\mathscr{C} \backslash \mathscr{C}_{a b c}$ jump $a b c$, we have $C \in \mathscr{C}_{a b c}$. Let $C_{a b}, C_{b c}$, and $C_{a c}$ be the $a b c$ parts of $C$. Let $D \in \mathscr{C}_{a b d}$. Let $D_{a b}, D_{b d}$, and $D_{a d}$ be the $a b d$-parts of $D$. We know that $D$ jumps $a b c$. If $D$ jumps both $a c d$ and $b c d$, then $\left(C-C_{a b}\right) \cdot D_{a b}$ and $\left(D-D_{a b}\right) \cdot C_{a b}$ are longest cycles (Figure 4.17(a)).

Then $\left(D-D_{a b}\right) \cdot C_{a b} \in \mathscr{C}_{a b d}$ but does not jump $a b c$, a contradiction. If $D$ jumps neither acd nor $b c d$, then $C_{a b} \cdot D_{a b}$ and $\left(C-C_{a b}\right) \cdot\left(D-D_{a b}\right)$ are both longest cycles (Figure 4.17(b)). But then $C_{a b} \cdot D_{a b}$ is a longest cycle that 2-crosses $V_{t}$, contradicting the fact that $\mathcal{X}_{2}=\varnothing$.

Hence,

$$
\text { every } D \in \mathscr{C}_{a b d} \text { jumps exactly one of }\{a c d, b c d\} \text {. }
$$

Analogously, we can conclude that

$$
\text { every } F \in \mathscr{C}_{a c d} \text { jumps exactly one of }\{b c d, a b d\} \text {, }
$$

and that

$$
\text { every } J \in \mathscr{C}_{b c d} \text { jumps exactly one of }\{a b d, a c d\} \text {. }
$$


Let $(D, F, J) \in \mathscr{C}_{a b d} \times \mathscr{C}_{a c d} \times \mathscr{C}_{b c d}$. Suppose that $D$ jumps $a c d$ and $F$ jumps abd. Then, by (4.2) and (4.3), both $\left(D-D_{a b}\right) \cdot C_{b c} \cdot F_{c a}$ and $\left(F-F_{a c}\right) \cdot C_{c b} \cdot D_{b a}$ are cycles, one of them longer than $L$, a contradiction (Figure $4.17(\mathrm{c})$ ).

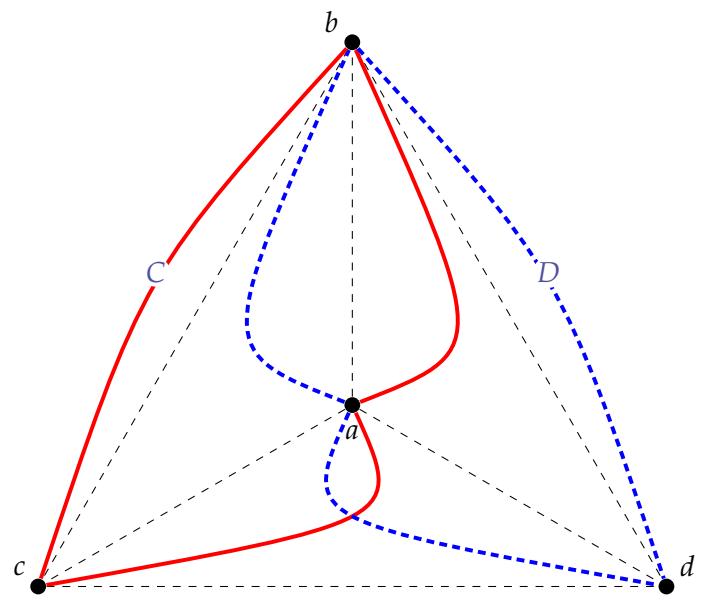

(a)

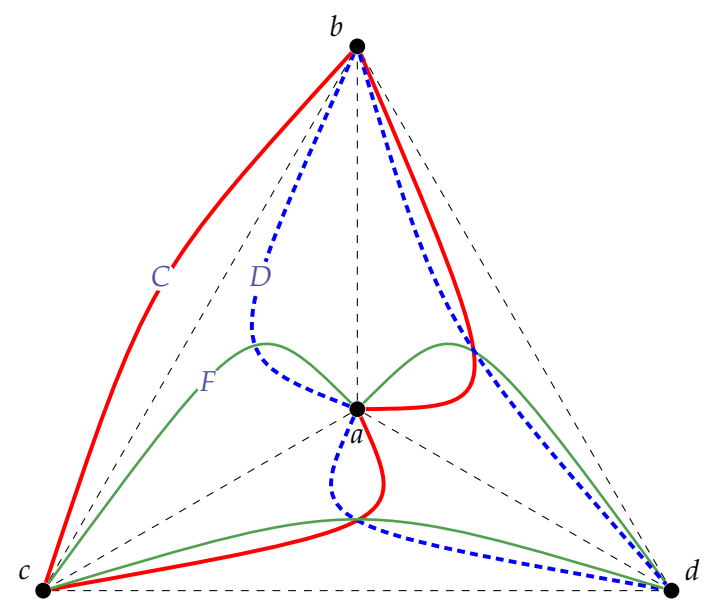

(c)

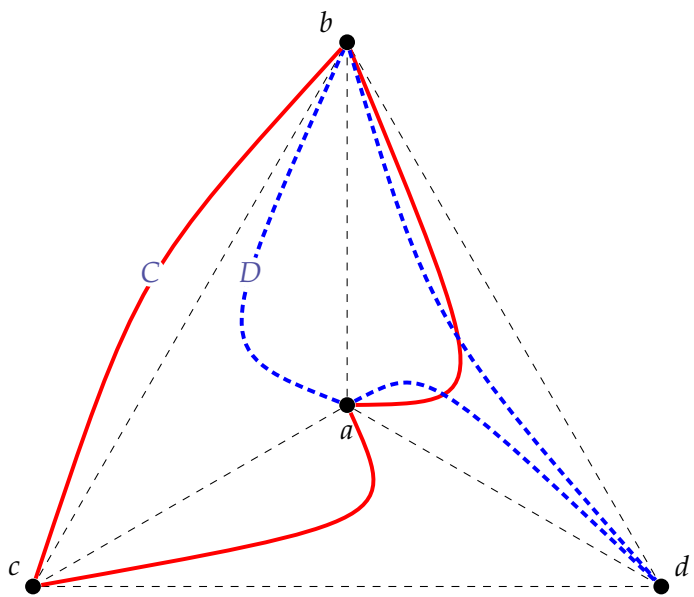

(b)

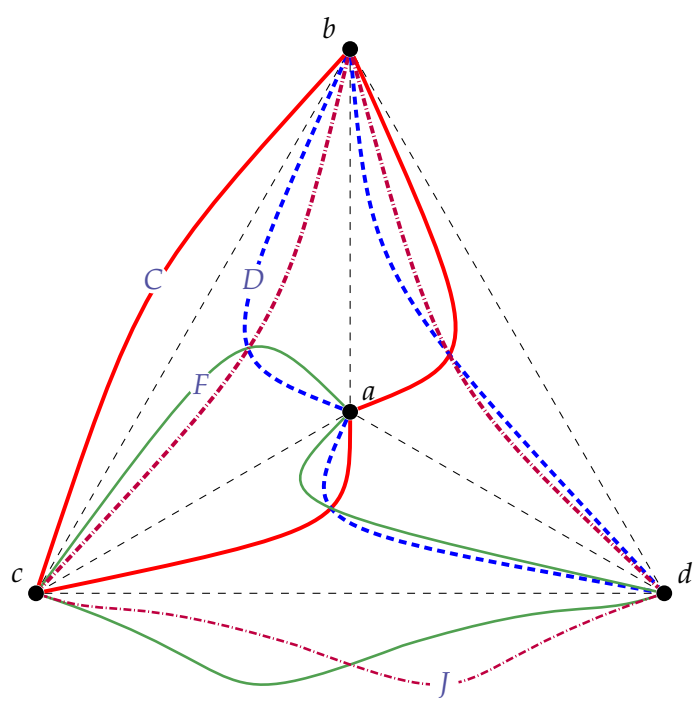

(d)

Figure 4.17: Case 1 in the proof of Claim 4.29 of Lemma 4.28 (a) $D$ jumps both acd and $b c d$. (b) $D$ does not jump neither $a c d$ nor $b c d$. (c) $D$ jumps acd and $F$ jumps $a b d$. (d) $D$ jumps $a c d$ and $F$ jumps $b c d$ and $J$ jumps $a b d$.

Repeating the same argument with $\{D, J\}$ and $\{F, J\}$, by (4.2), 4.3), and (4.4), we conclude that, without loss of generality,

$D$ jumps $a c d, F$ jumps $b c d$, and $J$ jumps $a b d$.

But then, $\left(D-D_{a b}\right) \cdot F_{a c} \cdot C_{c b},\left(F-F_{a c}\right) \cdot J_{c b} \cdot C_{b a}$, and $\left(J-J_{b c}\right) \cdot D_{b a} \cdot C_{a c}$ are cycles 
(Figure 4.17(d)), one of them longer than $L$, a contradiction.

Case 2: $C$ 4-intersects $V_{t}$.

Without loss of generality, suppose that $C$ has $a b d c$-parts, $C_{a b}, C_{b d}, C_{d c}$, and $C_{c a}$. As $C$ is outside $a b c$, we have that $C_{a b}$ is inside $a b d$ and $C_{a c}$ is inside $a c d$.

We show that

$$
\text { every cycle in } \mathscr{C}_{a b d} \text { does not jump acd. }
$$

Let $D \in \mathscr{C}_{a b d}$. Let $D_{a b}, D_{b d}$, and $D_{a d}$ be the corresponding abd-parts of $D$. Suppose by contradiction that $D$ jumps $a c d$. If $D_{b d}$ is outside $a b d$, then $\left(D-D_{a b}\right) \cdot C_{a b}$ and $\left(C-C_{a b}\right) \cdot D_{a b}$ are both longest cycles. Then $\left(D-D_{a b}\right) \cdot C_{a b} \in \mathscr{C}_{a b d}$ but does not jump $a b c$, a contradiction (Figure 4.18 (a)). If $D_{b d}$ is inside $a b d$, then both $C_{a b} \cdot C_{b d} \cdot D_{d a}$ and $\left(D-D_{a d}\right) \cdot C_{a c} \cdot C_{c d}$ are longest cycles and $C_{a b} \cdot C_{b d} \cdot D_{d a} \in \mathscr{C}_{a b d}$ but does not jump $a b c$, a contradiction (Figure $4.18(\mathrm{~b})$ ).

By symmetry, we can also show that

$$
\text { every cycle in } \mathscr{C}_{a c d} \text { does not jump abd. }
$$

Let $(D, F) \in \mathscr{C}_{a b d} \times \mathscr{C}_{a c d}$. Let $D_{a b}, D_{b d}$, and $D_{a d}$ be the corresponding $a b d$-parts of $D$. Let $F_{a c}, F_{c d}$, and $F_{a d}$ be the corresponding acd-parts of $F$. By (4.5) and (4.6), $D_{a d}$ is outside $a c d$, and $F_{a d}$ is outside $a b d$. This implies that $\left(D-D_{a d}\right) \cdot F_{a d}$ and $\left(F-F_{a d}\right) \cdot D_{a d}$ are both longest cycles. Then $\left(D-D_{a d}\right) \cdot F_{a d} \in \mathscr{C}_{a b d}$ and jumps $a c d$, a contradiction to 4.5) (Figure 4.18(c)).

This concludes the proof of the Claim.

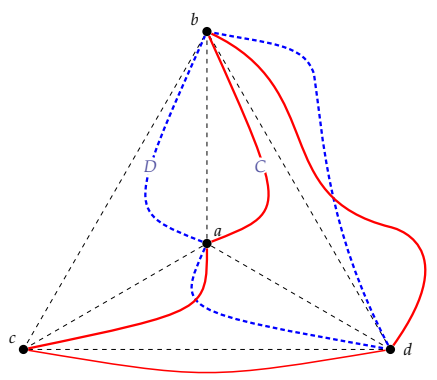

(a)

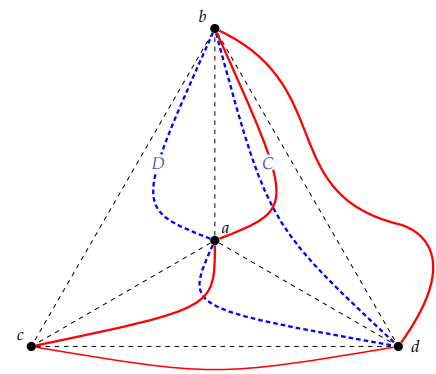

(b)

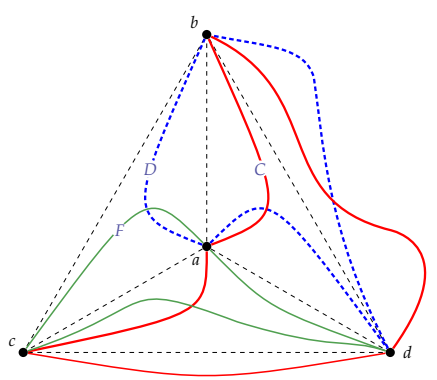

(c)

Figure 4.18: Case 2 in the proof of Claim 4.29. (a) $D$ jumps $a c d$ and $D_{b d}$ is outside $a b d$. (b) $D$ jumps $a c d$ and $D_{b d}$ is inside $a b d$. (c) $D$ does not jump acd and $F$ does not jump $a b d$.

The next claim is used in the proofs of Claims 4.31 and 4.32 . It uses Claim 4.29 .

Claim 4.30. For every triple of vertices $\Delta \subseteq V_{t}$, every cycle in $\mathscr{C}_{\Delta}$ jumps at least two of $\{a b d, a c d, b c d, a b c\} \backslash\{\Delta\}$. 
Proof of Claim 4.30 Suppose that $\Delta=a b c$ and there exists a cycle, say $C \in \mathscr{C}_{a b c}$, that jumps at most one of $\{a b d, a c d, b c d\}$. If $C$ jumps no one of $\{a b d, a c d, b c d\}$, then $C$ is inside $a b c$. Let $C_{a b}, C_{b c}$, and $C_{a c}$ be the $a b c$-parts of $C$. By Claim 4.29, there exists a longest cycle $D \in \mathscr{C} \backslash \mathscr{C}_{a b c}$ that does not jump abc. Without loss of generality, suppose that $D \in \mathscr{C}_{a b d}$ and let $D_{a b}, D_{b d}$, and $D_{a d}$ be the $a b d$-parts of $D$. Then $C_{a b} \cdot D_{a b}$ and $\left(C-C_{a b}\right) \cdot\left(D-D_{a b}\right)$ are both longest cycles, but $C_{a b} \cdot D_{a b} \in \mathcal{X}_{2}$, a contradiction. So, without loss of generality suppose $C$ jumps $a b d$. This means that

$$
C_{b c}, C_{a c} \text { are inside } a b c \text {, and } C_{a b} \text { is inside } a b d \text {. }
$$

Let $D \in \mathscr{C}_{a b d}$. Suppose for a moment that $D$ jumps abc. That is,

$$
D_{a b} \text { is inside } a b c \text {. }
$$

Note that $\left|C_{a b}\right|,\left|D_{a b}\right|>1$. Then, by (4.7) and 4.8), we have that $C_{a b} \cdot D_{a b}$ and $\left(C-C_{a b}\right) \cdot\left(D-D_{a b}\right)$ are both longest cycles. Hence, $C_{a b} \cdot D_{a b}$ is a longest cycle that 2-crosses $V_{t}$, contradicting the fact that $\mathcal{X}_{2}=\varnothing$ (Figure 4.19(a)). Hence, $D$ is outside $a b c$. Thus, as both $\left(C-C_{a b}\right) \cdot D_{a b}$ and $\left(C-C_{a b}\right) \cdot\left(D-D_{a b}\right)$ are cycles, we conclude that

$$
\left|C_{a b}\right| \geq L / 2
$$

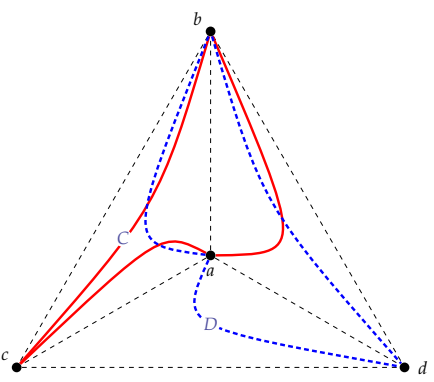

(a)

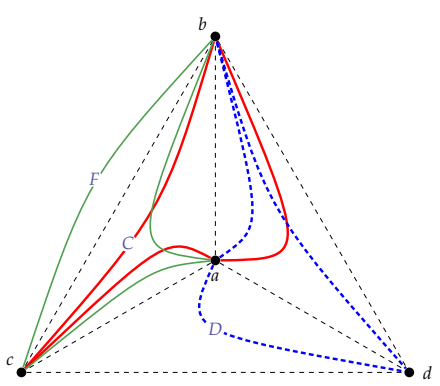

(b)

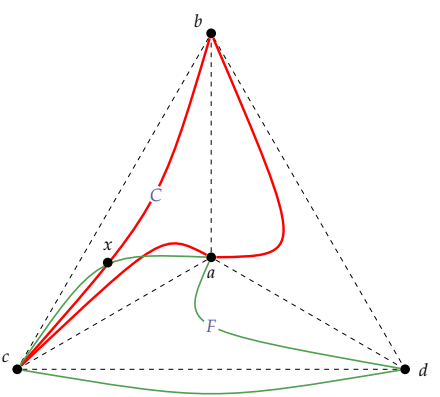

(c)

Figure 4.19: Cases in the proof of Claim 4.30 of Lemma 4.28. (a) $D$ jumps $a b c$. (b) $D$ is outside $a b c$ and $F \in \mathscr{C}_{a b c}$. (c) $D$ is outside $a b c$ and $F \in \mathscr{C}_{a c d}$.

By Claim 4.29, there exists a longest cycle $F \in \mathscr{C} \backslash \mathscr{C}_{a b d}$ that is outside $a b d$. If $F \in \mathscr{C}_{a b c}$ then both $C_{a b} \cdot F_{a b}$ and $C_{a b} \cdot\left(F-F_{a b}\right)$ are cycles. By (4.9), they are both longest cycles. But then $C_{a b} \cdot F_{a b}$ is a longest cycle that 2-crosses $V_{t}$, contradicting the fact that $\mathcal{X}_{2}=\varnothing$ (Figure 4.19 (b)). If $F \in \mathscr{C}_{a c d}$ then there exists a subpath $R$ of $C_{b c}$ starting at $b$, that ends at a vertex $x$ of $F_{a c}$ and is internally disjoint from $F$. Let $F_{x a}$ be the subpath of $F$ that is inside $a b c$ with extremes $x$ and $a$. Then, by (4.9), either $C_{a b} \cdot R \cdot F_{x a}$ or $C_{a b} \cdot R \cdot\left(F-F_{x a}\right)$ is a cycle longer than $L$, a contradiction (Figure 4.19 (c)). 
The case in which $F \in \mathscr{C}_{b c d}$ is symmetric to the case in which $F \in \mathscr{C}_{a c d}$. So, every cycle in $\mathscr{C}_{a b c}$ jumps at least two of $\{a b d, a c d, b c d\}$. Analogously, for every triple of vertices $\Delta \subseteq V_{t}$, every cycle in $\mathscr{C}_{\Delta}$ jumps at least two of $\{a b d, a c d, b c d, a b c\} \backslash\{\Delta\}$.

Claim 4.31. There exists a triple of vertices $\Delta \subseteq V_{t}$ such that every cycle in $\mathscr{C}_{\Delta}$ jumps exactly two of $\{a b d, a c d, b c d, a b c\} \backslash\{\Delta\}$.

Proof of Claim 4.31. By Claim 4.30, for each triple of vertices $\Delta \subseteq V_{t}$, every cycle in $\mathscr{C}_{\Delta}$ jumps two or three of $\{a b d, a c d, b c d, a b c\} \backslash\{\Delta\}$. Suppose by contradiction that each triple of vertices $\Delta \subseteq V_{t}$ is such that there exists a longest cycle in $\mathscr{C}_{\Delta}$ that jumps the three of $\{a b d, a c d, b c d, a b c\} \backslash\{\Delta\}$. Let $C, D$, and $F$ be corresponding cycles in $\mathscr{C}_{a b c}, \mathscr{C}_{a b d}$, and $\mathscr{C}_{b c d}$ respectively. Let $C_{a b}, C_{b c}$, and $C_{a c}$ be the $a b c$-parts of $C$. Let $D_{a b}, D_{b d}$, and $D_{a d}$ be the $a b d$-parts of $D$. Let $F_{b c}, F_{c d}$, and $F_{b d}$ be the $b c d$-parts of $F$. Observe that $\left(F-F_{b c}\right) \cdot C_{b c}$ and $\left(C-C_{b c}\right) \cdot F_{b c}$ are cycles, hence

$$
\left|F_{b c}\right|=\left|C_{b c}\right|
$$

Observe that $\left(F-F_{b d}\right) \cdot D_{b d}$ and $\left(D-D_{b d}\right) \cdot F_{b d}$ are cycles, hence

$$
\left|D_{b d}\right|=\left|F_{b d}\right|
$$

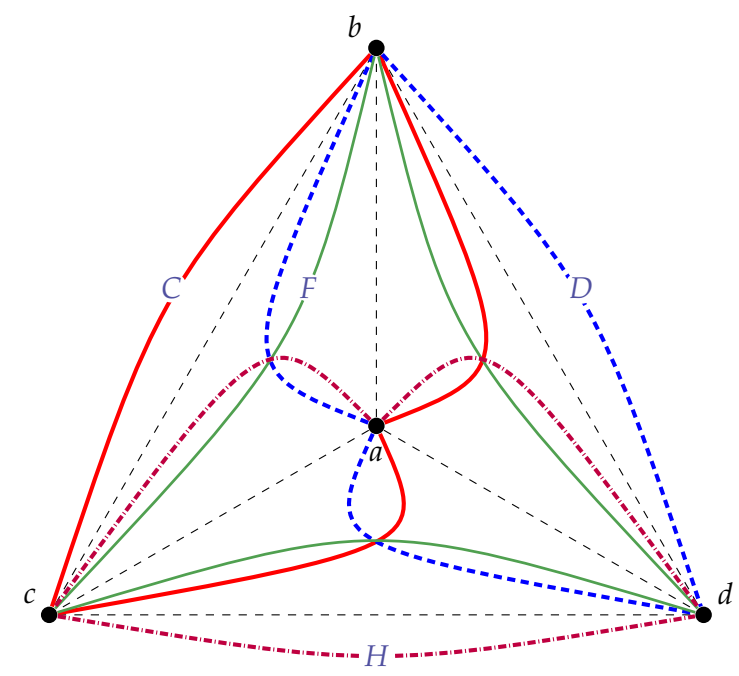

Figure 4.20: Situation in the proof of Claim 4.31 of Lemma 4.28

Let $H$ be a corresponding cycle in $\mathscr{C}_{a c d}$. Let $H_{a c}, H_{c d}$, and $H_{a d}$ be the acd-parts of $H$. We have that both $F_{c b} \cdot C_{b a} \cdot D_{a d} \cdot H_{d c}$ and $F_{d b} \cdot D_{b a} \cdot C_{a c} \cdot H_{c d}$ are cycles, and their lengths sum more than $\left|F_{b c}\right|+\left|F_{b d}\right|+\left|C_{a b}\right|+\left|C_{a c}\right|+\left|D_{a b}\right|+\left|D_{a d}\right|$. By (4.10) and (4.11), this sum equals $\left|C_{b c}\right|+\left|D_{b d}\right|+\left|C_{a b}\right|+\left|C_{a c}\right|+\left|D_{a b}\right|+\left|D_{a d}\right|=|C|+|D|=2 L$, a contradiction (Figure 4.20). 
Suppose that $a b c$ is the triangle given by Claim 4.31. Let $C \in \mathscr{C}_{a b c}$ (which exists because, by assumption, $\mathscr{C}_{a b c} \neq \varnothing$ ). As $C$ jumps exactly two of $\{a b d, a c d, b c d\}$, we may assume without loss of generality that $C$ jumps $a b d$ and $a c d$, and is outside $b c d$.

This means that

$$
C_{b c} \text { is inside } a b c, C_{a c} \text { is inside } a c d \text {, and } C_{a b} \text { is inside } a b d \text {. }
$$

By Claim 4.29 there exist cycles $F, H, J \in \mathscr{C}$ such that $F$ 2-intersects $a b d$ but does not jump $a b d, H$ 2-intersects $a c d$ but does not jump $a c d$, and $J$ 2-intersects $a b c$ but does not jump abc.

Claim 4.32. $F \in \mathscr{C}_{b c d}$ and $H \in \mathscr{C}_{b c d}$ (Figure 4.21 d)).

Proof of Claim 4.32 Suppose that $F \in \mathscr{C}_{a b c}$. By Claim 4.30, $F$ jumps both acd and $b c d$. If $H \in \mathscr{C}_{b c d}$ then, by Claim 4.30. $H$ jumps both $a b c$ and $a b d$. Note that $\left|F_{b c}\right|,\left|H_{b c}\right|>1$. Hence, $F_{b c} \cdot H_{b c}$ and $\left(F-F_{b c}\right) \cdot\left(H-H_{b c}\right)$ are both longest cycles. But then $F_{b c} \cdot H_{b c} 2-$ crosses $V_{t}$, a contradiction (Figure 4.21(a)). If $H \in \mathscr{C}_{a b d}$, by Claim 4.30. $H$ jumps both $a b c$ and $b c d$. Note that $\left|C_{a b}\right|,\left|H_{a b}\right|>1$. Hence, both $C_{a b} \cdot H_{a b}$ and $\left(C-C_{a b}\right) \cdot\left(H-H_{a b}\right)$ are longest cycles. But then $C_{a b} \cdot H_{a b}$ 2-crosses $V_{t}$, a contradiction. If $H \in \mathscr{C}_{a b c}$ then, by Claim 4.30, $H$ crosses both $a b d$ and $b c d$. Without loss of generality, we may assume that $J \in \mathscr{C}_{a b d}$. So, by Claim 4.30, $J$ jumps both $a c d$ and $b c d$. As both $\left(J-J_{a b}\right) \cdot F_{a b}$ and $\left(F-F_{a b}\right) \cdot J_{a b}$ are cycles, we have that $\left|F_{a b}\right|=\left|J_{a b}\right|$. Also, $\left(J-J_{a b}\right) \cdot C_{a b}$ is a cycle, so $\left|F_{a b}\right|=\left|J_{a b}\right| \geq\left|C_{a b}\right|$. As $\left(C-C_{a b}\right) \cdot J_{a b}$ is also a cycle, we have that $\left|C_{a b}\right| \geq\left|J_{a b}\right|$, concluding that $\left|C_{a b}\right|=\left|J_{a b}\right|=\left|F_{a b}\right|$. But then $\left(F-F_{a b}\right) \cdot C_{a b}$ is a cycle in $\mathscr{C}_{a b c}$ that jumps the three of $\{a b d, a c d, b c d\}$, a contradiction to the choice of $a b c$ according to Claim 4.31 (Figure 4.21(b)).

Now suppose that $F \in \mathscr{C}_{a c d}$. Then, by Claim 4.30, $F$ jumps abc. Note that $\left|F_{a c}\right|,\left|C_{a c}\right|>1$. Hence, both $F_{a c} \cdot C_{a c}$ and $\left(F-F_{a c}\right) \cdot\left(C-C_{a c}\right)$ are longest cycles. But $F_{a c} \cdot C_{a c} 2$-crosses $V_{t}$, a contradiction (Figure 4.21(c)). We conclude that $F \in \mathscr{C}_{b c d}$. By a similar argument, we also conclude that $H \in \mathscr{C}_{b c d}$ (Figure 4.21(d)).

The rest of the proof of Lemma 4.28 is divided on two cases depending on $J$.

Case 1: $J \in \mathscr{C}_{b c d}$ (Figure 4.22(a)).

Let $J_{b c}, J_{c d}$, and $J_{b d}$ be the $b c d$-parts of $J$. As $J_{b c}$ is outside $a b c$, we have that both $\left(J-J_{b c}\right) \cdot C_{b c}$ and $\left(C-C_{b c}\right) \cdot J_{b c}$ are longest cycles. But then $\left(C-C_{b c}\right) \cdot J_{b c}$ is a cycle in $\mathscr{C}_{a b c}$ that jumps the three of $\{a b d, a c d, b c d\}$, a contradiction to the choice of $C$ as in Claim 4.31 .

Case 2: $J \in \mathscr{C}_{a b d}$ (Figure $\left.4.22(\mathrm{~b})\right)$. 


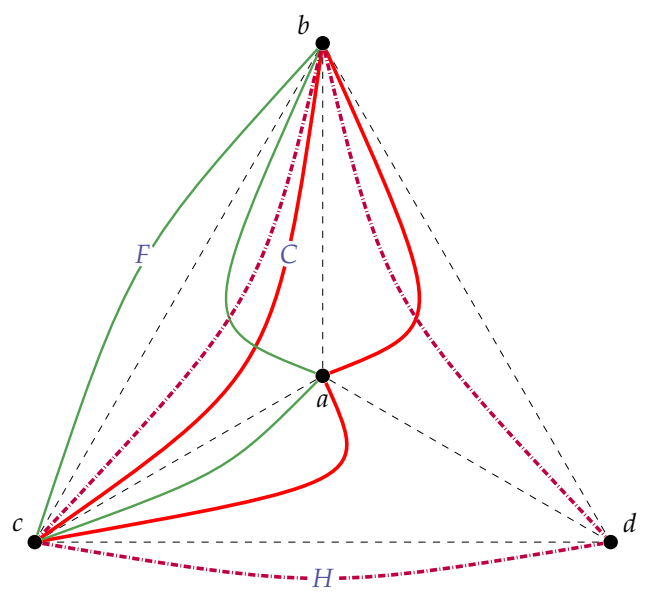

(a)

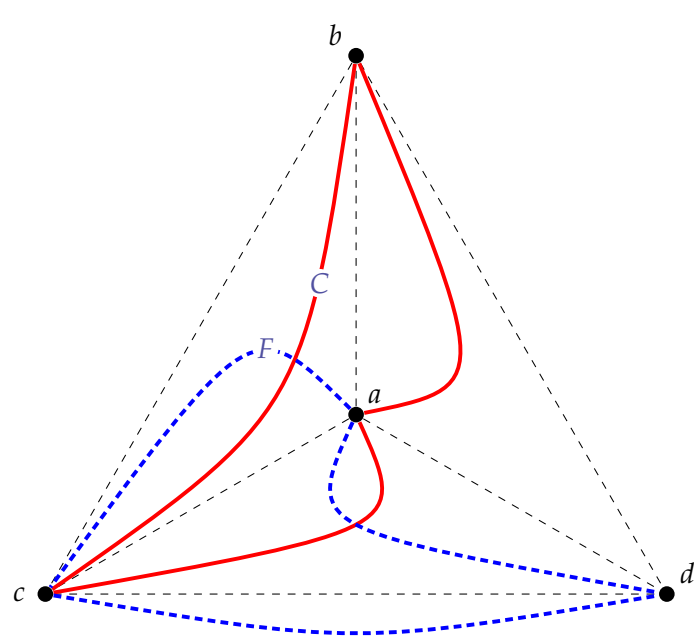

(c)

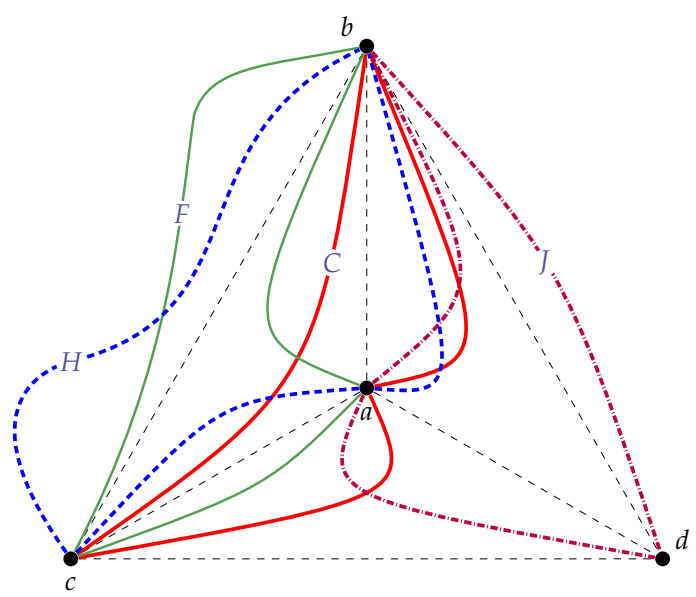

(b)

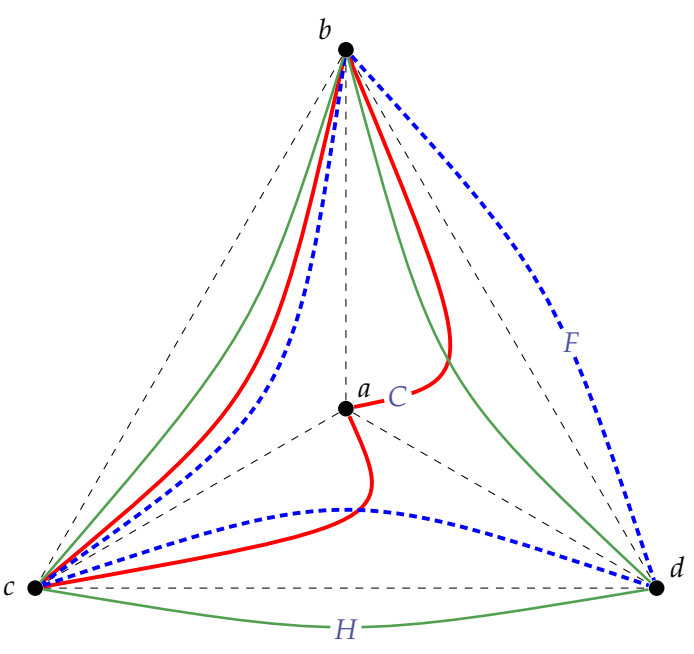

(d)

Figure 4.21: Cases in the proof of Claim 4.32 of Lemma 4.28. (a) $F \in \mathscr{C}_{a b c}$ and $H \in \mathscr{C}_{b c d}$. (b) $F, H \in \mathscr{C}_{a b c}$ and $J \in \mathscr{C}_{a b d}$. (c) $F \in \mathscr{C}_{a c d}$. (d) $F, H \in \mathscr{C}_{b c d}$.

Let $J_{a b}, J_{b d}$, and $J_{a d}$ be the $a b d$-parts of $J$. By Claim 4.30, J jumps both $a c d$ and $b c d$. As $\left(C-C_{a c}\right) \cdot H_{c d} \cdot J_{d a}$ is a cycle, we have that

$$
\left|C_{a c}\right| \geq\left|H_{c d}\right|+\left|J_{a d}\right|
$$

As both $\left(C-C_{a b}\right) \cdot J_{a b}$ and $\left(J-J_{a b}\right) \cdot C_{a b}$ are cycles, we have that $\left|C_{a b}\right|=\left|J_{a b}\right|$. Moreover,

$$
\left|J_{b d}\right|+\left|J_{a d}\right|=\left|C_{a c}\right|+\left|C_{b c}\right|
$$

By (4.12) and 4.13), we conclude that

$$
\left|J_{b d}\right| \geq\left|C_{b c}\right|+\left|H_{c d}\right|
$$


As both $\left(F-F_{b d}\right) \cdot J_{b d}$ and $\left(J-J_{b d}\right) \cdot F_{b d}$ are cycles, we have that

$$
\left|F_{b d}\right|=\left|J_{b d}\right|
$$

As both $\left(F-F_{b c}\right) \cdot C_{b c}$ and $\left(C-C_{b c}\right) \cdot F_{b c}$ are cycles, we have that

$$
\left|C_{b c}\right|=\left|F_{b c}\right|
$$

As $\left(H-H_{c d}\right) \cdot F_{c d}$ is a cycle, we have that

$$
\left|H_{c d}\right| \geq\left|F_{c d}\right|
$$

By (4.14), (4.15), (4.16), and (4.17), we conclude that $\left|F_{b d}\right| \geq\left|F_{b c}\right|+\left|F_{c d}\right|$. Hence,

$$
\left|F_{b d}\right| \geq L / 2
$$

Now, let $R$ be the subpath of $F_{c d}$ that is internally disjoint from $C_{a c}$, starts at $d$ and ends at a vertex $x$ in $C_{a c}$. Let $S$ be the subpath of $C$ that starts at $x$, ends at $b$, and is such that $|S| \geq L / 2$. Then, by (4.18), $F_{b d} \cdot R \cdot S$ is a cycle longer than $L$, a contradiction.

This concludes the proof of the lemma.

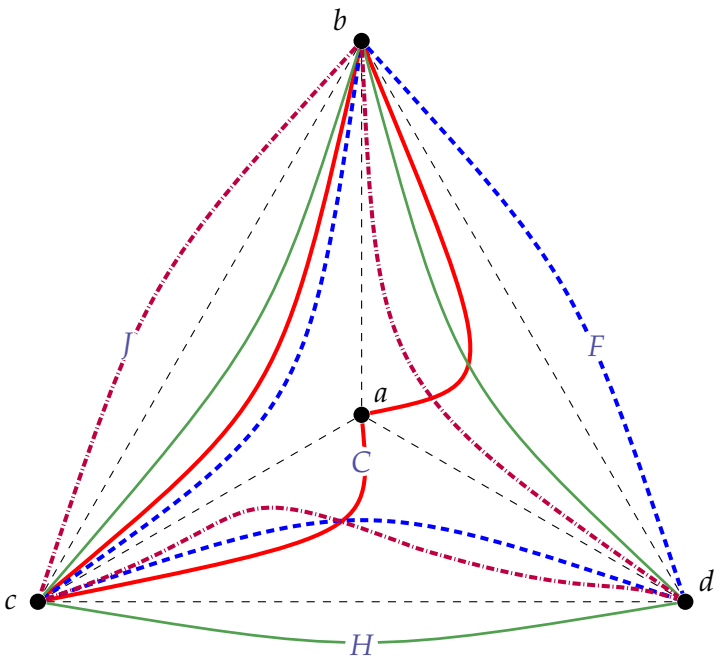

(a)

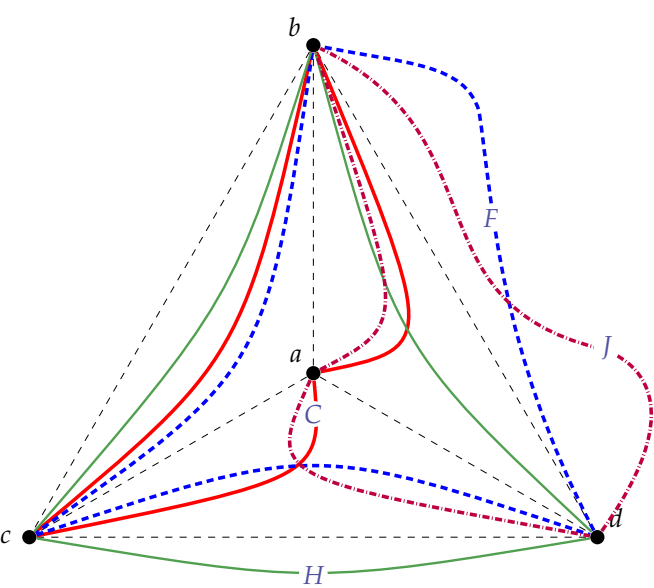

(b)

Figure 4.22: Cases in the last part of the proof of Lemma 4.28, (a) $J \in \mathscr{B}_{b c d}$ (Case 1). (b) $J \in \mathscr{B}_{a b d}$ (Case 2). 


\subsubsection{Proof of the auxiliary lemmas}

For all lemmas, propositions and corollaries in this subsection, we fix a graph $G$ with $\operatorname{tw}(G)=3$, a full tree decomposition $(T, \mathcal{V})$ of $G$, and a node $t \in V(T)$ with $V_{t}=\{a, b, c, d\}$.

We begin by extending the definition of branch. Given a component $A$ of $G-V_{t}$, we say that $\operatorname{Branch}_{t}(A)=\operatorname{Branch}_{t}(v)$, where $v$ is a vertex in $A$. Observe that $\operatorname{Branch}_{t}(A)$ is well defined. Indeed, as every pair of vertices in $A$ are not separated by $V_{t}$, we can apply part $(b)$ of Proposition 2.6 to every such pair.

Given a triple of vertices $\Delta \subseteq V_{t}$, it is denoted by $\mathcal{A}_{t}(\Delta)$ the set of components inside $\Delta$, that is,

$$
\mathcal{A}_{t}(\Delta)=\left\{A: A \text { is a component of } G-V_{t} \text { and } \operatorname{Branch}_{t}(A) \subseteq \mathscr{B}_{t}(\Delta)\right\}
$$

(Recall the definition of $\mathscr{B}_{t}(\Delta)$ in subsection 4.4.2.)

Lemma 4.33. For every ij $\in\{a b, b c, a c\}$, let $\mathscr{C}_{i j}$ be the collection of all longest cycles in $G$ that 2-jump abc at $\{i, j\}$. Let $\mathscr{C}_{a b c}$ be the collection of all longest cycles in $G$ that 3-jump abc. Let $\mathscr{C}=\mathscr{C}_{a b} \cup \mathscr{C}_{b c} \cup \mathscr{C}_{a c} \cup \mathscr{C}_{a b c}$. If $\mathscr{C}_{i j} \neq \varnothing$ for every $i j \in\{a b, b c, a c\}$, then all longest cycles in $\mathscr{C}$ pairwise intersect each other in a component of $\mathcal{A}_{t}(a b c)$.

Proof. Let $(\bar{C}, \bar{D}) \in \mathscr{C}_{a b} \times \mathscr{C}_{a c}$. Let $\bar{C}^{\prime}$ and $\bar{C}^{\prime \prime}$ be the two $a b$-parts of $\bar{C}$. Let $\bar{D}^{\prime}$ and $\bar{D}^{\prime \prime}$ be the two $a c$-parts of $\bar{D}$. As $\bar{C}$ and $\bar{D}$ jump $a b c$, we may assume that $\bar{C}^{\prime}$ and $\bar{D}^{\prime}$ are inside $a b c$ and that $\bar{C}^{\prime \prime}$ and $\bar{D}^{\prime \prime}$ are outside $a b c$. Suppose for a moment that $\bar{C}^{\prime}$ and $\bar{D}^{\prime}$ are internally disjoint. Let $F \in \mathscr{C}_{b c}$, which exists by the hypothesis of the lemma. Let $F^{\prime}$ and $F^{\prime \prime}$ be the two $b c$-parts of $F$. As $F$ jumps $a b c$, we may assume that $F^{\prime}$ is inside $a b c$ and that $F^{\prime \prime}$ is outside $a b c$. Let $R$ be a subpath of $F^{\prime}$ that is internally disjoint from $\bar{C}^{\prime}$ and $\bar{D}^{\prime}$ connecting $\bar{C}^{\prime}$ and $\bar{D}^{\prime}$. Observe that such a subpath exists because $V\left(F^{\prime}\right) \cap V\left(\bar{C}^{\prime}\right) \neq \varnothing$ and $V\left(F^{\prime}\right) \cap V\left(\bar{D}^{\prime}\right) \neq \varnothing$. Moreover, $|R|>0$ because $a \notin V(F)$ and $V\left(\bar{C}^{\prime}\right) \cap V\left(\bar{D}^{\prime}\right)=\{a\}$. Suppose that $V(R) \cap V(\bar{C})=\{x\}$ and that $V(R) \cap V(\bar{D})=\{y\}$. Then $\bar{C}^{\prime \prime} \cdot \bar{C}_{b x}^{\prime} \cdot R \cdot \bar{D}_{y a}^{\prime}$ and $\bar{D}^{\prime \prime} \cdot \bar{D}_{c y}^{\prime} \cdot R \cdot \bar{C}_{x a}^{\prime}$ are both cycles, one of them longer than $L$, a contradiction (Figure 4.23(a)). Thus $\bar{C}^{\prime}$ and $\bar{D}^{\prime}$ internally intersect inside $a b c$. As $\bar{C}^{\prime}$ and $\bar{D}^{\prime} 2$ intersects $a b c$, they are fenced by $a b c$. Hence, as they internally intersect each other, there exists a component $A \in \mathcal{A}_{t}(a b c)$ such that all internal vertices of $\bar{C}^{\prime}$ and $\bar{D}^{\prime}$ are in $A$. We show that $A$ is the desired component. That is, all longest cycles in $\mathscr{C}$ pairwise intersect each other in $A$. 


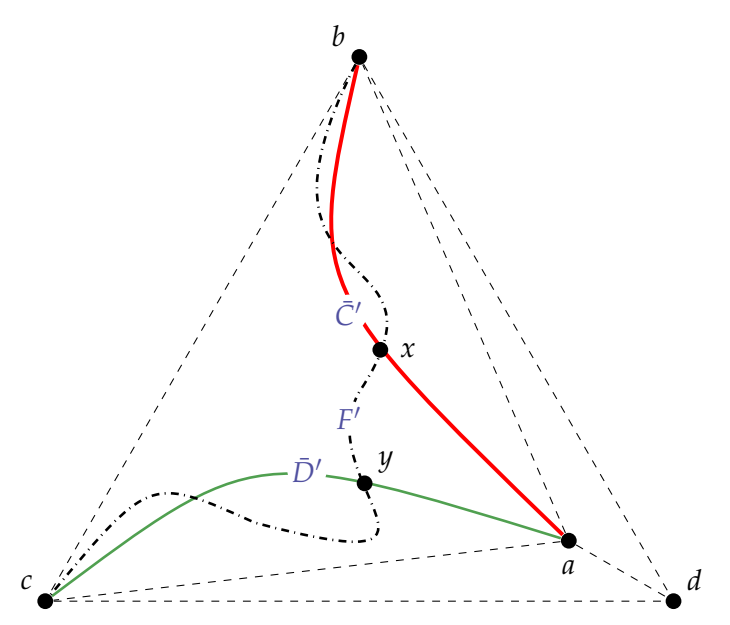

(a)

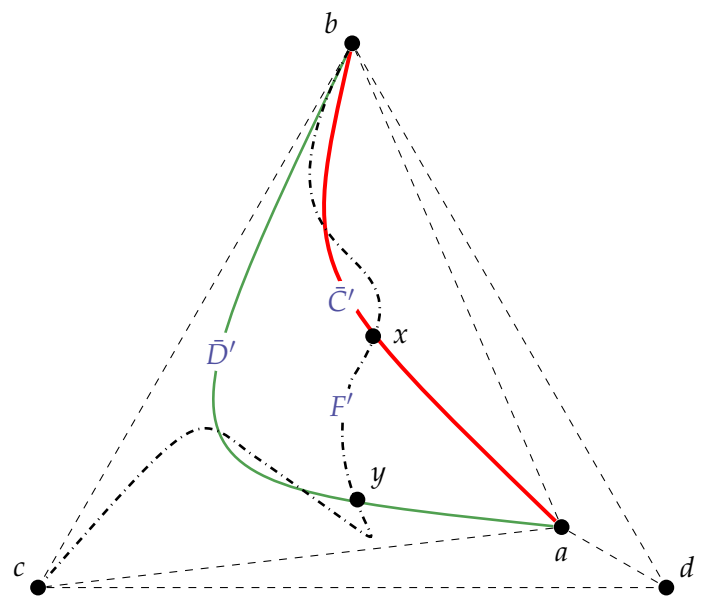

(b)

Figure 4.23: Cases in the proof of Lemma 4.33 (a) Pair $(\bar{C}, \bar{D}) \in \mathscr{C}_{a b} \times \mathscr{C}_{a c}$ with $\bar{C}^{\prime}$ and $\bar{D}^{\prime}$ inside $a b c$ and the path $F^{\prime}$. (b) Pair $(C, D) \in \mathscr{C}_{a b} \times \mathscr{C}_{a b}$ with $C^{\prime}$ and $D^{\prime}$ inside $a b c$ and the path $F^{\prime}$.

Let $(C, D) \in \mathscr{C}_{a b} \times \mathscr{C}_{a c}$. Let $C^{\prime}$ and $C^{\prime \prime}$ be the two $a b$-parts of $C$. Let $D^{\prime}$ and $D^{\prime \prime}$ be the two ac-parts of $D$. Analogously to the previous paragraph, $C^{\prime}$ and $D^{\prime}$ internally intersect inside $a b c$. Also, $C^{\prime}$ and $\bar{D}^{\prime}$ internally intersect inside $a b c$. Hence the internal vertices of $C^{\prime}$ and $D^{\prime}$ are in $A$. We conclude that $C^{\prime}$ and $D^{\prime}$ intersect in a vertex of $A$. A similar proof shows that every pair of cycles in $\left(\mathscr{C}_{a b} \times \mathscr{C}_{b c}\right) \cup\left(\mathscr{C}_{b c} \times \mathscr{C}_{a c}\right)$ intersect each other in a vertex of $A$.

Let $(C, D) \in \mathscr{C}_{a b} \times \mathscr{C}_{a b}$. Let $C^{\prime}$ and $C^{\prime \prime}$ be the two $a b$-parts of $C$. Let $D^{\prime}$ and $D^{\prime \prime}$ be the two $a b$-parts of $D$. As $C$ and $D$ jump $a b c$, we may assume that $C^{\prime}$ and $D^{\prime}$ are inside $a b c$ and that $C^{\prime \prime}$ and $D^{\prime \prime}$ are outside $a b c$. By the previous paragraph, $C^{\prime}$ and $\bar{D}^{\prime}$ internally intersect in $A$, and $D^{\prime}$ and $\bar{D}^{\prime}$ internally intersect in $A$. Hence, all internal vertices of $C^{\prime}$ and $D^{\prime}$ are in $A$. So, it suffices to prove that $C^{\prime}$ and $D^{\prime}$ internally intersect inside $a b c$. Suppose for a moment that $C^{\prime}$ and $D^{\prime}$ are internally disjoint. Let $F \in \mathscr{C}_{b c}$, which exists by the hypothesis of the lemma. Let $F^{\prime}$ and $F^{\prime \prime}$ be the two $b c$-parts of $F$. As $F$ jumps $a b c$, we may assume that $F^{\prime}$ is inside $a b c$ and that $F^{\prime \prime}$ is outside $a b c$. Let $R$ be a subpath of $F^{\prime}$ that is internally disjoint from $C^{\prime}$ and $D^{\prime}$ connecting $C^{\prime}$ and $D^{\prime}$. Observe that such a subpath exists because $F^{\prime}$ internally intersects $C^{\prime}$ and $D^{\prime}$ by the previous paragraph. Let $\{x\}=V(R) \cap V\left(C^{\prime}\right)$ and $\{y\}=V(R) \cap V\left(D^{\prime}\right)$. Note that $|R|>0$ because $a \notin V(F)$. Then $C^{\prime \prime} \cdot C_{b x}^{\prime} \cdot R \cdot D_{y a}^{\prime}$ and $D^{\prime \prime} \cdot D_{b y}^{\prime} \cdot R \cdot C_{x a}^{\prime}$ are both cycles, one of them longer than $L$, a contradiction (Figure 4.23(b)). A similar proof shows that every pair of cycles in $\left(\mathscr{C}_{b c} \times \mathscr{C}_{b c}\right) \cup\left(\mathscr{C}_{a c} \times \mathscr{C}_{a c}\right)$ intersect in $A$.

Let $(C, D) \in \mathscr{C}_{a b c} \times \mathscr{C}_{a b}$. Let $C_{a b}, C_{b c}$, and $C_{a c}$ be the $a b c$-parts of $C$. Let $D^{\prime}$ and $D^{\prime \prime}$ be the two $a b$-parts of $D$, with $D^{\prime}$ inside $a b c$. By the previous paragraph, $D^{\prime}$ and $\bar{C}^{\prime}$ internally intersect in $A$. Hence, all internal vertices of $D^{\prime}$ are in $A$. So, it suffices 
to prove that $D^{\prime}$ internally intersects any of $\left\{C_{a b}, C_{b c}, C_{a c}\right\}$. Suppose for a moment that $D^{\prime}$ does not internally intersect any of $\left\{C_{a b}, C_{b c}, C_{a c}\right\}$. As $C$ jumps $a b c$, at least one of $\left\{C_{a b}, C_{b c}, C_{a c}\right\}$ is inside $a b c$. If $C_{a b}$ is inside $a b c$ then $C_{a c} \cdot C_{c b} \cdot D^{\prime}$ and $D^{\prime \prime} \cdot C_{a b}$ are longest cycles. Indeed, $D^{\prime}$ is internally disjoint from $C_{a c}$ and $C_{c b}$, and $C_{a b}$ is internally disjoint from $D^{\prime \prime}$. As $D^{\prime \prime} \cdot C_{a b} \in \mathscr{C}_{a b}$, by the previous paragraph, $D^{\prime \prime} \cdot C_{a b}$ intersects $D$ in a vertex of $A$. As $D^{\prime \prime}$ is outside $a b c, C_{a b}$ and $D^{\prime}$ internally intersect in $A$, a contradiction. Hence $C_{a b}$ is outside $a b c$. Let $F \in \mathscr{C}_{b c}$, and let $F^{\prime}$ be the $b c$-part of $F$ that is inside $a b c$. Let $R$ be a subpath of $F^{\prime}$ internally disjoint from $D^{\prime}$ and $C$, connecting $D^{\prime}$ and $C$. Such a subpath exists because $F^{\prime}$ and $D^{\prime}$ internally intersect and $c \in V\left(F^{\prime}\right) \cap V(C)$. Let $\{x\}=V(R) \cap V\left(D^{\prime}\right)$ and $\{y\}=V(R) \cap V(C)$. As at least one of $\left\{C_{b c}, C_{a c}\right\}$ is inside $a b c$, we may assume (the proof for the other case is similar) that $y \in V\left(C_{b c}\right)$ (observe that possibly $y=c$ but $x \neq b$ ). Note that $|R|>0$. Let $C_{b y}$ and $C_{y c}$ be the two $y$-tails of $C_{b c}$. Let $D_{b x}^{\prime}$ and $D_{x a}^{\prime}$ be the two $x$-tails of $D^{\prime}$. Then $C_{a b} \cdot D_{b x}^{\prime} \cdot R \cdot C_{y c} \cdot C_{c a}$ and $D^{\prime \prime} \cdot C_{b y} \cdot R \cdot D_{x a}^{\prime}$ are cycles, one of them longer than $L$, a contradiction (Figure 4.24(a)). Hence, every pair of cycles in $\mathscr{C}_{a b c} \times \mathscr{C}_{a b}$ intersect in $A$. A similar proof shows that every pair of cycles in $\left(\mathscr{C}_{a b c} \times \mathscr{C}_{b c}\right) \cup\left(\mathscr{C}_{a b c} \times \mathscr{C}_{a c}\right)$ intersect in $A$.

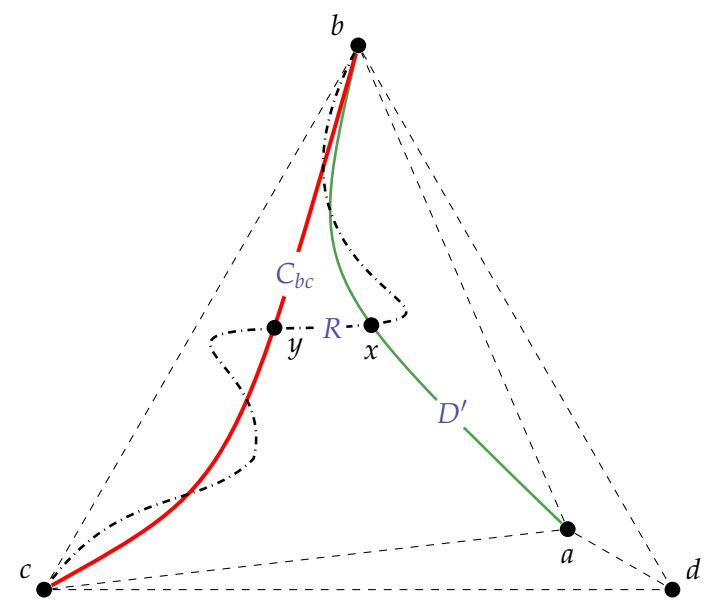

(a)

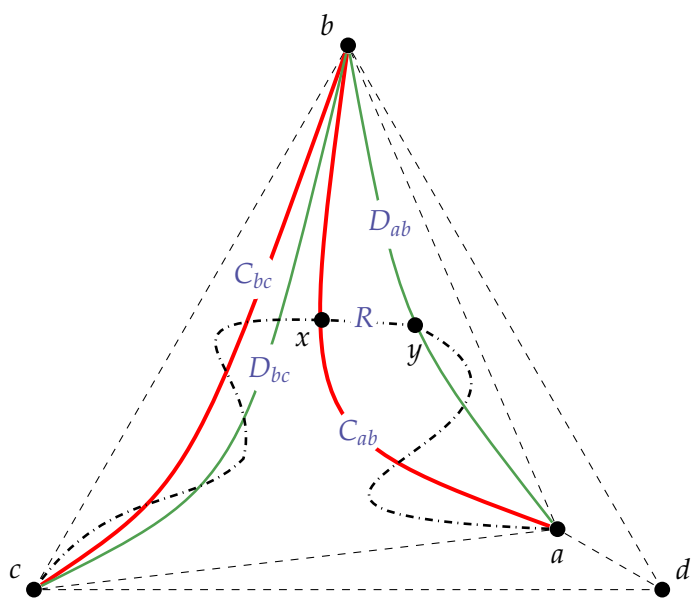

(b)

Figure 4.24: Cases in the proof of Lemma 4.33 (a) Pair $(C, D) \in \mathscr{C}_{a b c} \times \mathscr{C}_{a b}$, with $C_{b c}$ and $D^{\prime}$ inside $a b c$, and the path $F^{\prime}$. (b) Pair $(C, D) \in \mathscr{C}_{a b c} \times \mathscr{C}_{a b c}$ with $C_{a b}, C_{b c}, D_{a b}$ and $D_{b c}$ inside $a b c$, and the path $F^{\prime}$.

Finally, let $(C, D) \in \mathscr{C}_{a b c} \times \mathscr{C}_{a b c}$. Let $C_{a b}, C_{b c}$, and $C_{a c}$ be the abc-parts of $C$. Let $D_{a b}, D_{b c}$, and $D_{a c}$ be the $a b c$-parts of $D$. Suppose that only one of $\left\{C_{a b}, C_{a c}, C_{b c}\right\}$, say $C_{a b}$, is inside $a b c$. Let $F \in \mathscr{C}_{a b}, F^{\prime}$ be the $a b$-part of $F$ that is inside $a b c$, and $F^{\prime \prime}$ be the $a b$-part of $F$ that is outside $a b c$. Then $C_{a c} \cdot C_{c b} \cdot F^{\prime}$ and $F^{\prime \prime} \cdot C_{a b}$ are longest cycles. By the previous paragraph, as $F^{\prime \prime} \cdot C_{a b} \in \mathscr{C}_{a b}, C_{a b}$ and $D$ intersect in a vertex of $A$. Hence, we may assume that two of $\left\{C_{a b}, C_{a c}, C_{b c}\right\}$ are inside $a b c$, and, analogously, two 
of $\left\{D_{a b}, D_{a c}, D_{b c}\right\}$ are inside $a b c$. Without loss of generality we have two cases.

First suppose that $C_{a b}, C_{b c}, D_{a b}$, and $D_{b c}$ are inside $a b c$. Let $F \in \mathscr{C}_{a c}$. Suppose for a moment that $C$ and $D$ do not intersect in $A$. As $F$ intersects $C$ and $D$ in $A$, there exists a subpath $R$ of $F$ inside $a b c$, internally disjoint from $C$ and $D$, that starts at a vertex $x$ of $C$ and finishes at a vertex $y$ of $D$, say $x \in V\left(C^{\prime}\right)$, where $C^{\prime} \in\left\{C_{a b}, C_{b c}\right\}$ and $y \in V\left(D^{\prime}\right)$, where $D^{\prime} \in\left\{D_{a b}, D_{b c}\right\}$. Then $\left(C-C_{b x}^{\prime}\right) \cdot R \cdot D_{y b}^{\prime}$ and $\left(D-D_{b y}^{\prime}\right) \cdot R \cdot C_{x b}^{\prime}$ are cycles, one of them longer than $L$, a contradiction (Figure $4.24(b)$.)

Now suppose that $\left\{C_{a b}, C_{b c}\right\}$ and $\left\{D_{a b}, D_{a c}\right\}$ are inside $a b c$. Let $F \in \mathscr{C}_{a b}$. Suppose for a moment that $C$ and $D$ do not intersect in $A$. As $F$ intersects $C$ and $D$ in $A$, there exists a subpath $R$ of $F$ inside $a b c$, internally disjoint from $C$ and $D$, that starts at a vertex $x$ of $C$ and finishes at a vertex $y$ of $D$. We have two cases. If $x \in C_{a b}$, then let $D^{\prime}$ be the element of $\left\{D_{a b}, D_{a c}\right\}$ that contains $y$, and let $D_{y a}^{\prime}$ be the subpath of $D^{\prime}$ with extremes $a$ and $y$. Let $C_{a x}$ be the subpath of $C_{a b}$ with extremes $a$ and $x$. In that case, $\left(C-C_{a x}\right) \cdot R \cdot D_{y a}^{\prime}$ and $\left(D-D_{a y}^{\prime}\right) \cdot R \cdot C_{x a}$ are cycles, one of them longer than $L$, a contradiction (Figure 4.25(a)). Finally, consider the case in which $x \in C_{b c}$. Let $C_{b x}$ be the subpath of $C_{b c}$ with extremes $b$ and $x$. If $y \in D_{a b}$, then $\left(C-C_{b x}\right) \cdot R \cdot D_{y b}$ and $\left(D-D_{b y}\right) \cdot R \cdot C_{x b}$ are cycles, where $D_{b y}$ is the subpath of $D_{a b}$ with extremes $b$ and $y$, a contradiction. If $y \in D_{a c}$, then $\left(C-C_{c x}\right) \cdot R \cdot D_{y c}$ and $\left(D-D_{c y}\right) \cdot R \cdot C_{x c}$ are cycles, where $D_{c y}$ is the subpath of $D_{a c}$ with extremes $c$ and $y$, again a contradiction (Figure 4.25(b)).

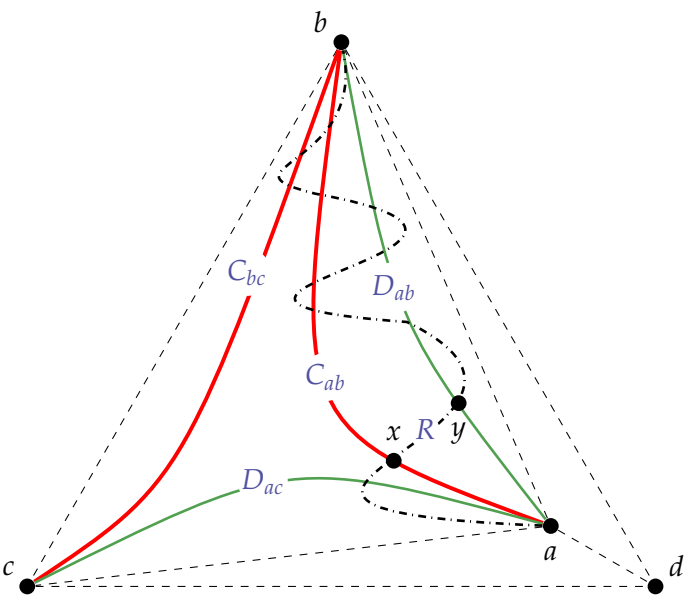

(a)

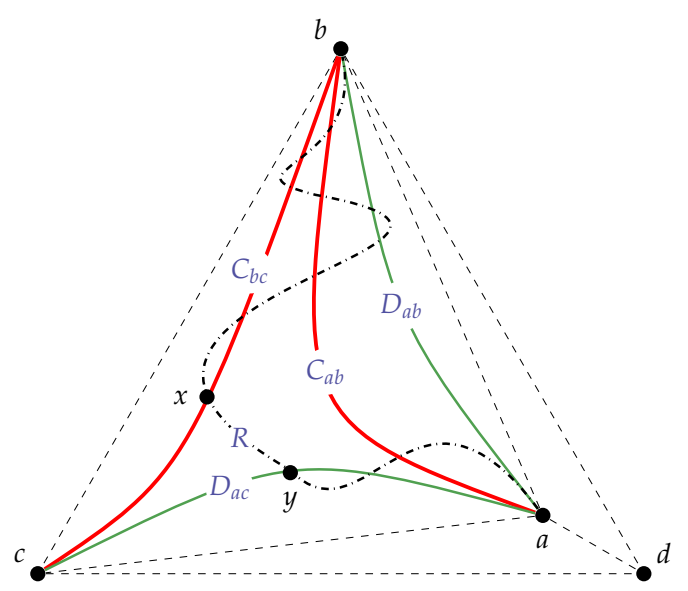

(b)

Figure 4.25: Pair $(C, D) \in \mathscr{C}_{a b c} \times \mathscr{C}_{a b c}$, with $C_{a b}, C_{b c}, D_{a b}$ and $D_{a c}$ inside $a b c$. (a) $x \in C_{a b}$ and $y \in D_{a b}$. (b) $x \in C_{b c}$ and $y \in D_{a c}$.

As $\mathscr{C} \times \mathscr{C}=\left(\mathscr{C}_{a b} \times \mathscr{C}_{a b}\right) \cup\left(\mathscr{C}_{a c} \times \mathscr{C}_{a c}\right) \cup\left(\mathscr{C}_{b c} \times \mathscr{C}_{b c}\right) \cup\left(\mathscr{C}_{a b} \times \mathscr{C}_{a c}\right) \cup\left(\mathscr{C}_{a b} \times \mathscr{C}_{b c}\right) \cup$ $\left(\mathscr{C}_{b c} \times \mathscr{C}_{a c}\right) \cup\left(\mathscr{C}_{a b c} \times \mathscr{C}_{a b}\right) \cup\left(\mathscr{C}_{a b c} \times \mathscr{C}_{a c}\right) \cup\left(\mathscr{C}_{a b c} \times \mathscr{C}_{b c}\right) \cup\left(\mathscr{C}_{a b c} \times \mathscr{C}_{a b c}\right)$, the proof follows. 
Given a vertex $v \notin V_{t}$, it is denoted by $T[v]$ the subtree of $T$ induced by $v$, that is $T[v]=T\left[\left\{t: v \in V_{t}\right\}\right]$. Given a component $A$ of $G-V_{t}$, it is denoted by $T[A]$ the subtree of $T$ induced by the vertices of $A$, that is, $T[A]=\cup_{v \in A} T[v]$. Note that $T[A]$ is indeed a tree as $T[A]$ is connected. It is denoted by $t^{*}(A)$ the node in $T[A]$ that is closest to $t$ in $T$. Observe that, as $T[A]$ is connected, this node is unique.

Proposition 4.34. Let $A$ be a component of $G-V_{t}$. If $a^{\prime}$ is a vertex in $A$ and $a a^{\prime} \in E(G)$, then $a \in V_{t^{*}}(A)$.

Proof. Let $t^{*}:=t^{*}(A)$. As $a a^{\prime} \in E(G)$, by the definition of tree decomposition, there exists a node $t^{\prime}$ such that $\left\{a, a^{\prime}\right\} \subseteq V_{t^{\prime}}$. As $a^{\prime} \in A$, by the definition of $T[A], t^{\prime} \in T[A]$. By the definition of $t^{*}$, the unique path from $t^{\prime}$ to $t$ in $T$ contains $t^{*}$. Hence, as $a \in V_{t} \cap V_{t^{\prime}}$, we conclude that $a \in V_{t^{*}}$.

Lemma 4.35. For every ij $\in\{a b, b c, a c\}$, let $\mathscr{C}_{i j}$ be the collection of all longest cycles in $G$ that 2-jump abc at $\{i, j\}$. Let $\mathscr{C}_{a b c}$ be the collection of all longest cycles in $G$ that 3-jump abc. Let $\mathscr{C}=\mathscr{C}_{a b} \cup \mathscr{C}_{b c} \cup \mathscr{C}_{a c} \cup \mathscr{C}_{a b c}$. If $\mathscr{C}_{i j} \neq \varnothing$ for every ij $\in\{a b, b c, a c\}$, then all cycles in $\mathscr{C}$ intersect at a common vertex $t$-inside abc.

Proof. By Lemma 4.33, there exists a component $A \in \mathcal{A}_{t}(a b c)$ such that all cycles in $\mathscr{C}$ pairwise intersect in $A$. Let $t^{*}:=t^{*}(A)$. As $\mathscr{C}_{i j} \neq \varnothing$ for every $i j \in\{a b, b c, a c\}$, there exists an $i j$-part $\bar{C}_{i j}$ of a cycle in $\mathscr{C}_{i j}$ such that all internal vertices of $\bar{C}_{i j}$ are in $A$, for every $i j \in\{a b, b c, a c\}$. Let $a^{\prime}$ be the neighbor of $a$ in $\bar{C}_{a b}, b^{\prime}$ be the neighbor of $b$ in $\bar{C}_{b c}$, and $c^{\prime}$ be the neighbor of $c$ in $\bar{C}_{a c}$. As $\left\{a^{\prime}, b^{\prime}, c^{\prime}\right\} \subseteq A$, by Proposition $4.34,\{a, b, c\} \subseteq V_{t^{*}}$. Let $V_{t^{*}}=\{a, b, c, f\}$. We proceed by induction on the number of vertices $t$-inside $a b c$ different from $a, b$, and $c$. If $f$ is the only vertex $t$-inside $a b c$ different from $a, b$, and $c$, then, as all cycles in $\mathscr{C}$ pairwise intersect in $A, f$ is in all cycles in $\mathscr{C}$ and the proof follows. So, there exist at least two vertices $t$-inside $a b c$ different from $a, b$, and $c$. Suppose by contradiction that there is no vertex in $A$ that belongs to all cycles in $\mathscr{C}$. Then, there exists (at least) one longest cycle in $\mathscr{C}$ that does not contain $f$. We divide the rest of the proof in two cases, depending on the type of this cycle.

Case 1: A longest cycle in $\mathscr{C}_{a b} \cup \mathscr{C}_{b c} \cup \mathscr{C}_{a c}$ does not contain $f$.

Let $C$ be such a cycle. Without loss of generality, assume that $C \in \mathscr{C}_{a b}$. Let $C^{\prime}$ and $C^{\prime \prime}$ be the two $a b$-parts of $C$. As $C t$-jumps $a b c$, we may assume that $C^{\prime}$ is $t$-inside $a b c$ and that $C^{\prime \prime}$ is $t$-outside $a b c$. Note that, as $C^{\prime}$ 2-intersects $V_{t^{*}}$ at $\{a, b\}$, and its extremes are in $V_{t^{*}}, C^{\prime}$ is fenced by $V_{t^{*}}$. Also, $C^{\prime}$ has at least one vertex not in $V_{t^{*}}$, so the internal vertices of $C^{\prime}$ are in a component, say $A^{\prime}$, of $G-V_{t^{*}}$. We show that $A^{\prime} \in \mathcal{A}_{t^{*}}(a b f)$. Let $t^{\prime}$ be the neighbor of $t^{*}$ such that $\operatorname{Branch}_{t^{*}}\left(A^{\prime}\right)=\operatorname{Branch}_{t^{*}}\left(t^{\prime}\right)$. By the definition of $\mathcal{A}_{t^{*}}(a b f)$, we must prove that $\{a, b, f\} \subseteq V_{t^{\prime}}$. Let $a^{\prime}$ be the neighbor of $a$ in $C^{\prime}$, and let $b^{\prime}$ be the neighbor of $b$ in $C^{\prime}$. As $\left\{a^{\prime}, b^{\prime}\right\} \subseteq A^{\prime}$, by Proposition 4.34, $\{a, b\} \subseteq V_{t^{\prime}}$. As $\left|V_{t^{\prime}} \cap V_{t^{*}}\right|=3$, 
either $c \in V_{t^{\prime}}$ or $f \in V_{t^{\prime}}$. If the former is true then, by Proposition 2.7, abc separates $f$ from $a^{\prime}$. But then $f$ and $a^{\prime}$ are not in the same component of $G-V_{t}$, a contradiction. Hence, $f \in V_{t^{\prime}}$ as we wanted.

Let $D \in \mathscr{C}$. As $C^{\prime}$ and $D$ intersect in a vertex not in $a b f$, and all the internal vertices of $C^{\prime}$ are in $A^{\prime}$, then $C^{\prime}$ and $D$ intersect in a vertex of $A^{\prime}$. Thus, $D$ has at least one abfpart $t^{*}$-inside $a b f$. As $D t$-jumps $a b c$, it also has an $a b c$-part $t$-outside $a b c$. This implies that $D$ has also an $a b f$-part that is $t^{*}$-outside $a b f$, so we conclude that $D t^{*}$-jumps $a b f$. Hence,

$$
\text { every cycle in } \mathscr{C} t^{*} \text { - jumps } a b f \text {. }
$$

Now we show that if $D \in \mathscr{C}_{a c}$, then $D t^{*}$-jumps $a b f$ at $\{a, f\}$. As $D t^{*}$-jumps $a b f$, there exists an $a b f$-part of $D$, say $D^{\prime}$, that is $t^{*}$-inside $a b f$. As $b \notin V(D)$, then $D^{\prime}$ is an $a f$-part of $D$. Let $D^{\prime \prime}$ be the other af-part of $D$. As $c \in V\left(D^{\prime \prime}\right)$ is a vertex $t^{*}$-outside $a b f, D^{\prime \prime}$ is $t^{*}$ outside $a b f$. Hence $D t^{*}$-jumps $a b f$ at $\{a, f\}$. Analogously, we can show that if $D \in \mathscr{C}_{b c}$, then $D t^{*}$-jump abf at $\{b, f\}$. We conclude that $\mathscr{C}_{i j} \neq \varnothing$ for every ij $\in\{a b, b f, a f\}$, where $\mathscr{C}_{i j}$ is the collection of all longest cycles that 2-jumps abf at $\{i, j\}$. Note that every vertex $t^{*}$-inside $a b f$ is also $t$-inside $a b c$. Hence, as $c$ is $t$-inside $a b c$ but not $t^{*}$-inside $a b f$, by induction hypothesis, there exists a common vertex to all cycles that $t^{*}$-jump $a b f$. Also, by 4.19), there exists a common vertex to all cycles in $\mathscr{C}$ (Figure 4.26(a)). Moreover, this vertex is $t$-inside $a b c$.

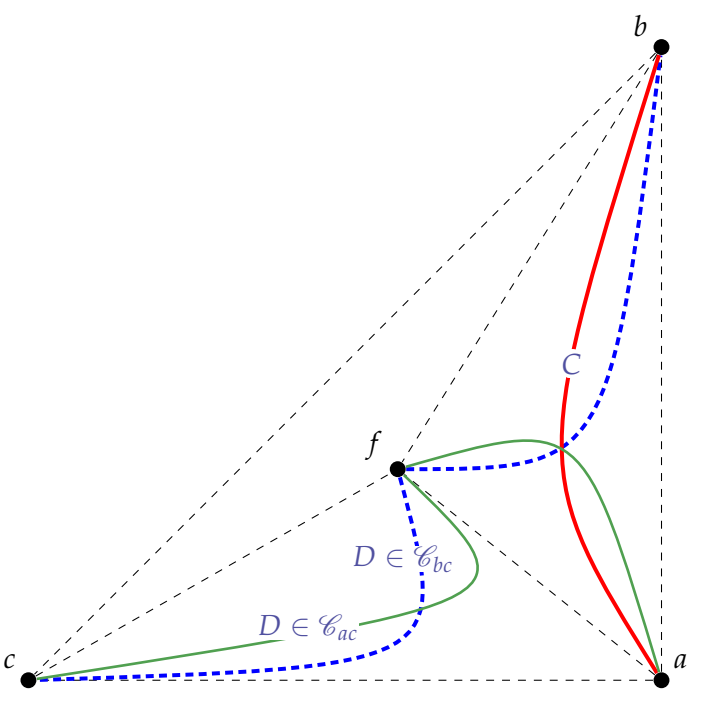

(a)

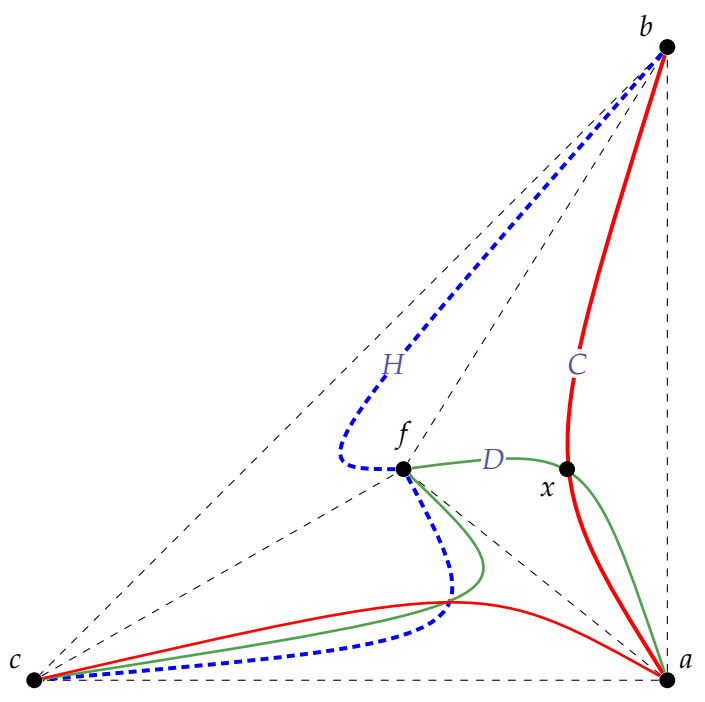

(b)

Figure 4.26: Cases in the proof of Lemma 4.35. (a) A longest cycle $C \in \mathscr{C}_{a b}$ that does not contain $f$ (Case 1). (b) A longest cycle $C \in \mathscr{C}_{a b c}$ that does not contain $f$ (Case 2.2), and every cycle in $\mathscr{C}_{b c} t^{\prime}$-jumps both $a b f$ and acf (Equation (4.21)).

Case 2: A longest cycle in $\mathscr{C}_{a b c}$ does not contain $f$. 
Let $C$ be such a cycle. Let $C_{a b}, C_{b c}$, and $C_{a c}$ be the corresponding $a b c$-parts of $C$. As $C$ has at least a vertex in $A$ (recall that $A$ is the component given by Lemma 4.33), at least one of $\left\{C_{a b}, C_{b c}, C_{a c}\right\}$ has all its internal vertices in $A$. Also, as $C t$-jumps $a b c$, at least one of $\left\{C_{a b}, C_{b c}, C_{a c}\right\}$ is $t$-outside $a b c$ (hence not in $A$ ).

Case 2.1: Exactly one of $\left\{C_{a b}, C_{b c}, C_{a c}\right\}$ has all its internal vertices in $A$.

Without loss of generality, let $C_{a b}$ be the corresponding part. Let $D \in \mathscr{C}_{a b}$ and $D^{\prime}$ be the $a b$-part of $D$ such that all internal vertices of $D^{\prime}$ are in $A$. Let $D^{\prime \prime}$ be the other $a b$-part of $D$. Then $D^{\prime} \cdot C_{a c} \cdot C_{c b}$ and $C_{a b} \cdot D^{\prime \prime}$ are both longest cycles. Observe that $C_{a b} \cdot D^{\prime \prime}$ is a longest cycle in $\mathscr{C}_{a b}$. But then $f$ is not in $C_{a b} \cdot D^{\prime \prime}$ and we are in Case 1.

Case 2.2: Exactly two of $\left\{C_{a b}, C_{b c}, C_{a c}\right\}$ have all their internal vertices in $A$.

Without loss of generality, let $C_{a b}$ and $C_{a c}$ be the corresponding parts. Let $A^{\prime}$ be the component of $G-V_{t^{*}}$ where the internal vertices of $C_{a b}$ lie. Let $A^{\prime \prime}$ be the component of $G-V_{t^{*}}$ where the internal vertices of $C_{a c}$ lie. As in Case 1, we can show that $A^{\prime} \in \mathcal{A}_{t^{*}}(a b f)$ and that $A^{\prime \prime} \in \mathcal{A}_{t^{*}}(a c f)$. Next we prove the following statements.

Every cycle in $\mathscr{C}_{a b}$ internally intersects $C_{a c}$ and every cycle in $\mathscr{C}_{a c}$ internally intersects $C_{a b}$,

every cycle in $\mathscr{C}_{b c} t^{*}$-jumps both $a b f$ and $a c f$,

every cycle in $\mathscr{C}_{a b} t^{*}$-jumps $a b f$ and every cycle in $\mathscr{C}_{a c} t^{*}$-jumps $a c f$,

every path that 2-intersects $V_{t^{*}}$ at $\{b, c\}$ is $t^{*}$-outside $b c f$.

Proof of 4.20): Let $D \in \mathscr{C}_{a b}$. Let $D^{\prime}$ and $D^{\prime \prime}$ be the two $a b$-parts of $D$ with $D^{\prime} t$ inside $a b c$ and $D^{\prime \prime} t$-outside $a b c$. If $D^{\prime}$ does not internally intersect $C_{a c}$, then both $D^{\prime \prime} \cdot C_{a b}$ and $D^{\prime} \cdot C_{a c} \cdot C_{c b}$ are longest cycles. Thus, $D^{\prime \prime} \cdot C_{a b}$ is a longest cycle in $\mathscr{C}_{a b}$ that does not contain $f$ and we would be in Case 1 . We conclude that $D^{\prime}$ internally intersects $C_{a c}$. A similar proof applies when $D \in \mathscr{C}_{a c}$.

Proof of (4.21): Let $H \in \mathscr{C}_{b c}$. We show that $H t^{*}$-jumps both $a b f$ and acf. Suppose that $H$ does not $t^{*}$-jump $a b f$. Let $D$ be a cycle in $\mathscr{C}_{a c}$. By (4.20), there exists an $a f$-part of $D$, say $D^{\prime}$, such that $D^{\prime}$ internally intersects $C_{a b}$. Let $R$ be the subpath of $D^{\prime}$ that is internally disjoint from $C_{a b}$, starts at $f$ and ends at a vertex $x \in V\left(C_{a b}\right)$. Let $C_{b x}$ be the subpath of $C_{a b}$ with extremes $b$ and $x$. Let $H_{b f}$ be the subpath of $H$ with extremes $b$ and $f$ that does not contain $c$. As $H_{b f}$ is $t^{*}$-outside $a b f$, we have two cycles, $\left(C-C_{b x}\right) \cdot R \cdot H_{f b}$ and $\left(H-H_{b f}\right) \cdot R \cdot C_{x b}$, one of them longer than $L$, a contradiction (Figure $\left.4.26(b)\right)$. The proof of the other case is symmetric. 
Proof of (4.22): Let $D \in \mathscr{C}_{a b}$. If $f \notin V(D)$, then we would be in Case 1. Hence $f \in V(D)$. Let $D_{a f}, D_{b f}$, and $D_{a b}$ be the $a b f$-parts of $D$. Suppose that $D$ does not $t^{*}$-jump $a b f$. Then $D_{b f}$ is internally disjoint from $C_{a b}$. Also, by (4.20), $D_{a f}$ is internally disjoint from $C_{a b}$. Let $H \in \mathscr{C}_{a c}$. By (4.20), $H$ internally intersects $C_{a b}$. Let $R$ be the subpath of $H$ that is internally disjoint from $C_{a b}$, starts at $f$ and ends at a vertex $x \in V\left(C_{a b}\right)$. Let $C_{b x}$ be the subpath of $C_{a b}$ with extremes $b$ and $x$. As $C_{b x}$ is internally disjoint from $D_{b f}$, we have two cycles, $\left(C-C_{b x}\right) \cdot R \cdot D_{f b}$ and $\left(D-D_{b f}\right) \cdot R \cdot C_{x b}$, a contradiction (Figure 4.27(a)). The proof for $\mathscr{C}_{a c}$ is symmetric.

Proof of (4.23): Suppose by contradiction that there exists a path $R$ that 2-intersects $V_{t^{*}}$ at $\{b, c\}$ and is $t^{*}$-inside $b c f$. Let $D \in \mathscr{C}_{a b}$ and $F \in \mathscr{C}_{a c}$. If $f \notin V(D)$, then we would be in Case 1. Hence $f \in V(D)$. Analogously, $f \in V(F)$. Let $D_{a f}, D_{b f}$, and $D_{a b}$ be the $a b f$-parts of $D$. Let $F_{a f}, F_{c f}$, and $F_{a c}$ be the $a c f$-parts of $F$. By $(4.20), D_{a f}$ is $t^{*}$-inside $a c f$ and $F_{a f}$ is $t^{*}$-inside $a b f$. By (4.20) and (4.22), we have that $D_{b f}$ and $F_{c f}$ do not internally intersect $R$. Then $D_{a b} \cdot R \cdot F_{c f} \cdot F_{f a}$ and $F_{a c} \cdot R \cdot D_{b f} \cdot D_{f a}$ are cycles, one of them longer than $L$, a contradiction (Figure $4.27(b)$ ).

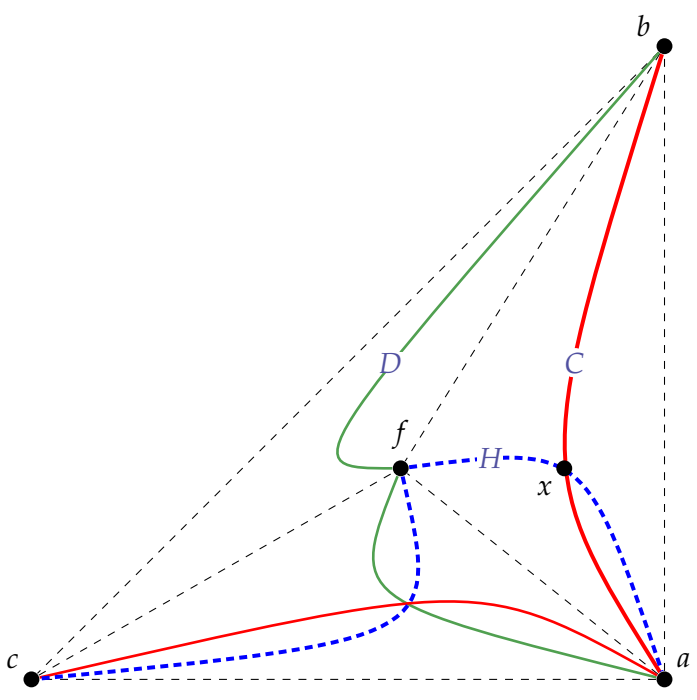

(a)

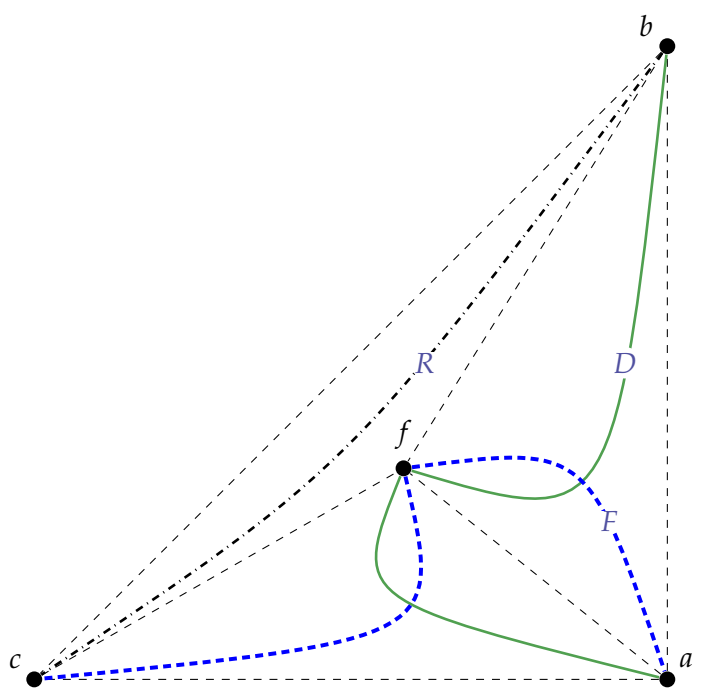

(b)

Figure 4.27: Situations in Case 2.2 of Lemma 4.35. We have a longest cycle $C \in \mathscr{C}_{a b c}$ that does not contain $f$. (a) We show that every cycle in $\mathscr{C}_{a b} t^{*}$-jumps $a b f$ and that every cycle in $\mathscr{C}_{a c} t^{*}$-jumps acf (Equation (4.22)). (b) We show that every path that 2-intersects $V_{t^{*}}$ at $\{b, c\}$ is $t^{*}$-outside $b c f$ (Equation 4.23).

We show that

$$
\text { every cycle in } \mathscr{C} t^{*} \text {-jump } a b f \text {. }
$$

Suppose by contradiction that this is not the case. Let $D$ be a cycle in $\mathscr{C}$ that does not $t^{*}$-jump $a b f$. By (4.20), if $D \in \mathscr{C}_{a c}$, then $D$ internally intersects $C_{a b}$, so $D$ jumps $a b f$. Therefore $D \notin \mathscr{C}_{a c}$. By (4.21), $D \notin \mathscr{C}_{b c}$. By (4.22), $D \notin \mathscr{C}_{a b}$. We conclude that $D \in \mathscr{C}_{a b c}$. 
Suppose for a moment that $f \notin V(D)$. As $D \in \mathscr{C}_{a b c}$, exactly two of $\left\{D_{a b}, D_{b c}, D_{a c}\right\}$ are $t$-inside $a b c$, otherwise we are in Case 2.1. By (4.23), we know that $D_{b c}$ is not $t^{*}$-inside $b c f$. Hence, both $D_{a b}$ and $D_{a c}$ are $t$-inside $a b c$, so $D_{a b}$ is $t^{*}$-inside $a b f$, and $D t^{*}$-jumps abf, a contradiction. We conclude that $f \in V(D)$.

As $D$ 3-intersects $a b c$, we have two cases, depending on the $a b f$-parts of $D$. First suppose that there is an abf-part of $D$, say $D^{\prime}$, such that the extremes of $D^{\prime}$ are $b$ and $f$, and $D^{\prime}$ does not contain $c$. Consider a cycle $H \in \mathscr{C}_{b c}$. If $f \notin V(H)$ then we are in Case 1. Hence, $f \in V(H)$. Let $H_{b c}, H_{c f}$, and $H_{b f}$ be the $b c f$-parts of $H$. By (4.21), $H_{b f}$ is $t^{*}$-inside $a b f$ and $H_{c f}$ is $t^{*}$-inside $a c f$. By (4.23), $H_{b c}$ is $t^{*}$-outside $b c f$. Recall that $D$ does not jump abf. Hence, $\left(D-D^{\prime}\right) \cdot H_{b f}$ and $\left(H-H_{b f}\right) \cdot D^{\prime}$ are longest cycles. Then $\left(H-H_{b f}\right) \cdot D^{\prime} \in \mathscr{C}_{b c}$ but does not $t^{*}$-jump abf, a contradiction to (4.21) (Figure 4.28(a)). Now suppose that there exists no such part of $D$. Hence $D_{a f}, D_{f c}, D_{c b}$, and $D_{b a}$ are the $a b c f$-parts of $D$, with $D=D_{a f} \cdot D_{f c} \cdot D_{c b} \cdot D_{b a}$. As $D \in \mathscr{C}_{a b c}$, exactly two of $\left\{D_{a b}, D_{a f} \cdot D_{f c}, D_{b c}\right\}$ are $t$-inside $a b c$, otherwise we are in Case 2.1. If $D_{a b}$ is $t$-inside $a b c$, then $D t^{*}$-jumps $a b f$ and we are done. Hence, $D_{b c}$ is $t$-inside $a b c$, a contradiction to (4.23) (Figure 4.28(b)).

By (4.20), 4.21), and 4.22, we have that $\mathscr{C}_{i j} \neq \varnothing$ for every ij $\in$ abf, where $\mathscr{C}_{i j}$ is the collection of all longest cycles that 2-jump abf at $\{i, j\}$. Note that every vertex $t^{*}$-inside $a b f$ is also $t$-inside $a b c$. Hence, as $c$ is $t$-inside $a b c$ but not $t^{*}$-inside $a b f$, by induction hypothesis, there exists a common vertex to all cycles that $t^{*}$-jump $a b f$. Also, by (4.24), there exists a common vertex to all cycles in $\mathscr{C}$. Moreover, this vertex is $t$-inside $a b c$.

This concludes the proof of the lemma.

Corollary 4.36. Let $\Delta$ be a triple of vertices in $V_{t}$. If $\operatorname{lct}(G)>1$ and for every pair of vertices $\{i, j\}$ in $\Delta$ there exists a longest cycle that 2-jumps $\Delta$ at $\{i, j\}$, then there exists a longest cycle $C$ in $G$ such that either $C$ intersects $V_{t}$ at most once, or one of the following possibilities is true:

(i) C is t-outside $\Delta$,

(ii) $C$ is t-inside $\Delta$ and 2-intersects $\Delta$,

(iii) $C$ is t-inside $\Delta, 3$-intersects $\Delta$, and is fenced by $\Delta$.

Proof. Without loss of generality, let $\Delta=a b c$. For every $i j \in\{a b, b c, a c\}$, let $\mathscr{C}_{i j}$ be the collection of all longest cycles in $G$ that 2-jump $a b c$ at $\{i, j\}$. Let $\mathscr{C}_{a b c}$ be the collection of all longest cycles in $G$ that 3-jump abc. Let $\mathscr{C}=\mathscr{C}_{a b} \cup \mathscr{C}_{b c} \cup \mathscr{C}_{a c} \cup \mathscr{C}_{a b c}$. As $\mathscr{C}_{i j} \neq \varnothing$ for every $i j \in\{a b, b c, a c\}$, by Lemma 4.35, all longest cycles in $\mathscr{C}$ intersect at a common vertex, say $x$, that is $t$-inside $a b c$. As $\operatorname{lct}(G)>1$, there exists a longest cycle $C$ in $G$ that is 


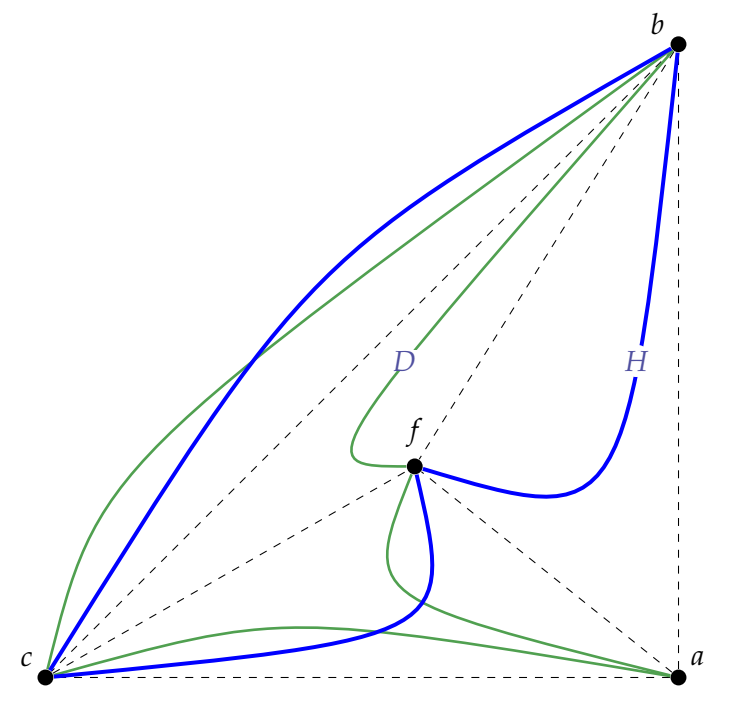

(a)

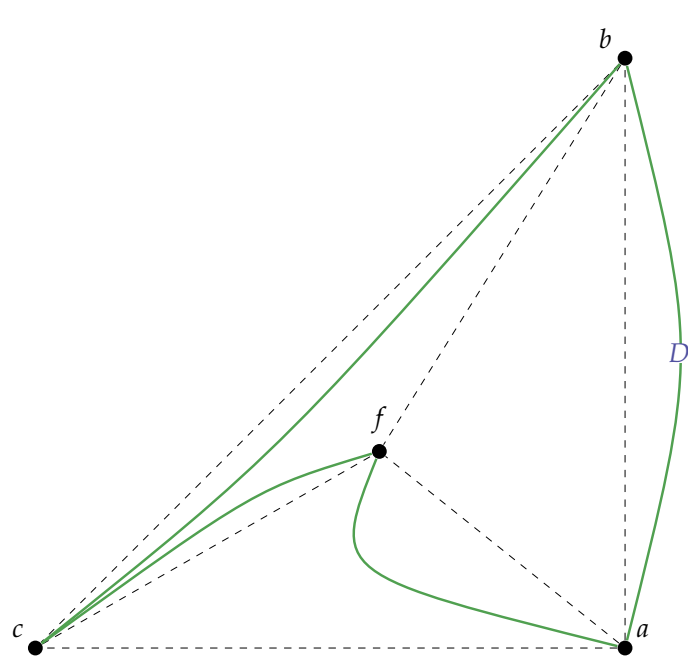

(b)

Figure 4.28: Cases in the last part of the proof of Lemma 4.35. A cycle $D \in \mathscr{C}_{a b c}$ that does not jump abf.

not in $\mathscr{C}$. Hence, $C$ does not jump $a b c$. We may assume that $C$ intersects $V_{t}$ at least twice. If $C$ is $t$-outside $a b c$, then $(i)$ holds and we are done. So, assume that $C$ is $t$-inside $a b c$. If $C$ 2-intersects $\Delta$, then (ii) holds and we are done. Hence, we may assume that $C$ 3-intersects $\Delta$.

Suppose by contradiction that $C$ crosses $\Delta$. Let $C_{a b}, C_{b c}$, and $C_{a c}$ be the $a b c$-parts of $C$. Let $A$ be the component of $G-\Delta$ where $x$ lies. As $C$ crosses $\Delta$, at least one of $\left\{C_{a b}, C_{b c}, C_{a c}\right\}$, say $C_{a b}$, has all its internal vertices not in $A$. Let $D \in \mathscr{C}_{a c}$. Let $F \in \mathscr{C}_{b c}$. Let $D^{\prime}$ and $D^{\prime \prime}$ be the two ac-parts of $D$. Let $F^{\prime}$ and $F^{\prime \prime}$ be the two $b c$-parts of $F$. As $D$ and $F$ jump $a b c$, we may assume that $D^{\prime}$ and $F^{\prime}$ are $t$-inside $a b c$, and that $D^{\prime \prime}$ and $F^{\prime \prime}$ are $t$-outside $a b c$. Moreover, as $D$ and $F$ contain $x$, and $x$ is in $A$, both $D^{\prime}$ and $F^{\prime}$ have all its internal vertices in $A$. As $C_{a b}$ has all its internal vertices not in $A, C_{a b}$ is internally disjoint from $D^{\prime}$ and $F^{\prime}$. As $C$ is t-inside $a b c, C_{a b}$ is internally disjoint from $D^{\prime \prime}$ and $F^{\prime \prime}$. Hence, $D^{\prime} \cdot C_{a b} \cdot F^{\prime \prime}$ and $F^{\prime} \cdot C_{a b} \cdot D^{\prime \prime}$ are both cycles, one of them longer than $L$, a contradiction.

\subsection{Remarks}

In this chapter we showed upper bounds for the minimum cardinality of a set of vertices that intersects all longest cycles in a 2-connected graph. A natural question asks for lower instead of upper bounds. It is known that it is not always the case that all longest cycles intersect in arbitrary 2-connected graphs, so there are 2-connected graphs $G$ such that $\operatorname{lct}(G)>1$. The question of whether $\operatorname{lct}(G)=1$ when $G$ is chordal is still open. There exists a 2-connected graph $G$ given by Thomassen on 15 vertices [SZZ13], with 
treewidth four and $\operatorname{lct}(G)=2$ (Figure 4.29 .

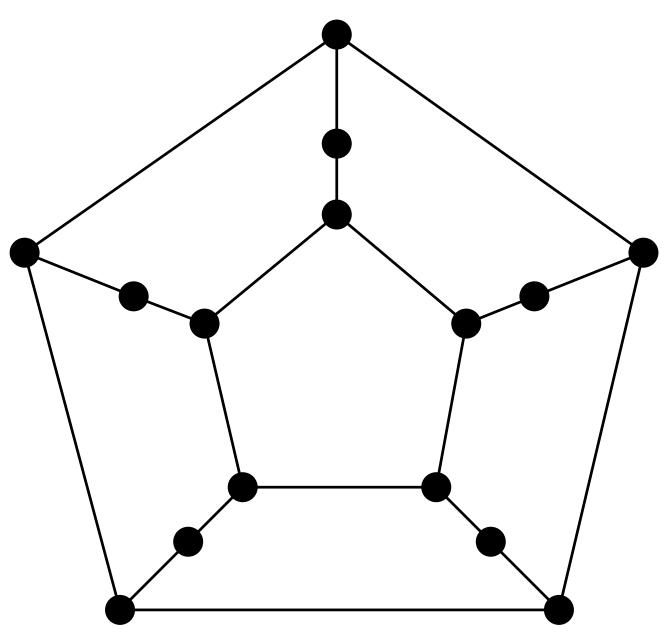

(a)

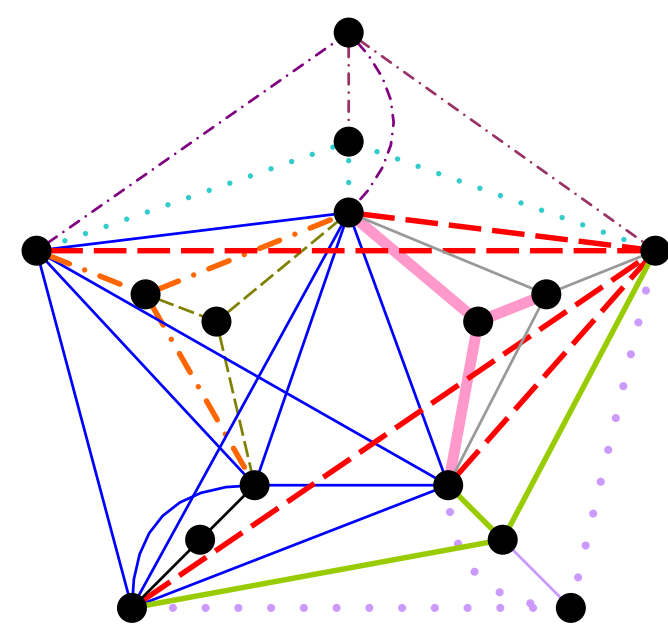

(b)

Figure 4.29: (a) Thomassen's graph. Calling it $G$, we have that $\operatorname{lct}(G)=2$. (b) A chordal completion of $G$. As $G$ itself is a forbidden minor for partial 3-trees [APC90], we conclude that $\operatorname{tw}(G)=4$.

Hence, by Theorem 4.18 , we conclude the following corollary.

Corollary 4.37. Let $\ell$ be the minimum integer such that $\operatorname{lct}(G) \leq \ell$ for every partial 4-tree $G$. Then, $\ell \in\{2,3\}$. 


\section{Chapter 5}

\section{Transversals of Triangles}

In this chapter we address transversals of triangles, and their relation with packings of edge-disjoint triangles, in some graph classes. Recall that $\tau(G)$ is the minimum cardinality of a triangle transversal of a given graph $G$ and that $v(G)$ is the maximum cardinality of a triangle packing of $G$. We prove the following:

- $\tau(G) \leq 2 v(G)$ for every partial 6-tree $G$ (Section 5.2.

- $\tau(G) \leq \frac{9}{5} v(G)+\frac{1}{5}$ for every 3-tree $G$ (Section 5.3.

- $\tau(G) \leq \frac{3}{2} v(G)$ for every planar triangulation $G$ different from $K_{4}$ (Section 5.4 ).

- $\tau(G) \leq \frac{n^{2}}{3 \cdot\left(4 m-n^{2}\right)} \cdot v(G)$ for every tripartite graph $G$ with $n$ vertices and $m \geq \frac{n^{2}}{4}$ edges (Section 5.5).

- $\tau(G) \leq \frac{2 a d+2 b c}{2 a d+a c-2 c-2 d} \cdot v(G)$ for every complete 4-partite graph $G$ with parts of cardinalities $a, b, c$, and $d, a \geq b \geq c \geq d$, and $a>1$ (Section 5.6).

Throughout all this chapter, we use the term transversal instead of triangle transversal, and packing instead of triangle packing. Before presenting our results, we introduce new concepts that are used in Sections 5.2 and 5.3 .

\subsection{Rooted Tree Decomposition}

A rooted tree is a pair $(T, r)$, where $T$ is a tree and $r$ is a vertex of $T$. Given $t \in V(T)$, it is denoted by $P_{T, r}(t)$ the unique path in $T$ that joins $r$ and $t$. If $t^{\prime}$ is a vertex in $V\left(P_{T, r}(t)\right)$, then we say that $t^{\prime}$ is an ancestor of $t$. Every vertex in $T$ that has $t$ as its ancestor is called a descendant of $t$. If $t \neq r$, then the parent of $t$, denoted by $p_{T, r}(t)$, is the ancestor of $t$ that is adjacent to $t$. The successors of $t$ are the vertices whose parent is $t$, and the set of successors of $t$ is denoted by $S_{T, r}(t)$. The height of $t$, denoted by $h_{T, r}(t)$, is the length of a longest path that joins $t$ to a descendant of $t$. When $(T, r)$ is clear from the context, we simply write $P(t), p(t), S(t)$, and $h(t)$.

Let $G$ be a graph. Let $(\mathcal{V}, T)$ be a tree decomposition of $G$ and let $r \in V(T)$. We say that $(\mathcal{V}, T, r)$ is a rooted tree decomposition if 
- $(\mathcal{V}, T)$ is a full tree decomposition; and

- if $t \in V(T) \backslash\{r\}$, then $V_{t} \cap V_{p(t)} \neq V_{t} \cap V_{t^{\prime}}$ for every $t^{\prime} \in S(t)$ (Figure 5.1).

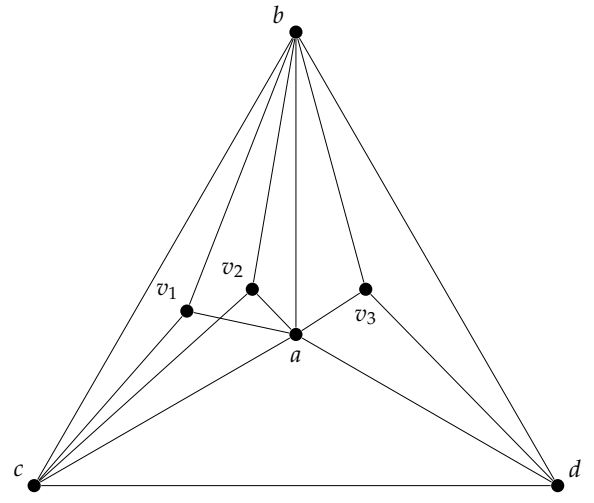

(a)

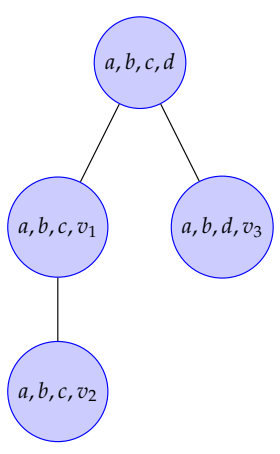

(b)

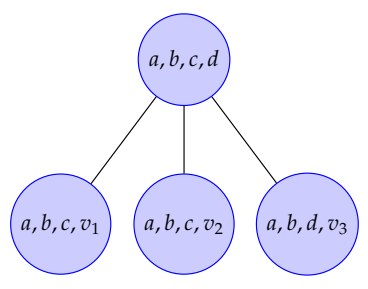

Figure 5.1: (a) A graph with treewidth 3. (b) Two full tree decompositions of the graph. Only the second is rooted.

Proposition 5.1. Every graph $G$ has a rooted tree decomposition of width $\mathrm{tw}(G)$.

Proof. By Proposition 2.1, there exists a full tree decomposition of $G$ of width $\operatorname{tw}(G)$. Let $(T, \mathcal{V}, r)$ be a triple that minimizes $\sum_{t \in V(T)}\left|P_{T, r}(t)\right|$, where $(T, \mathcal{V})$ is a full tree decomposition of $G$ of width $\operatorname{tw}(G)$, and $r \in V(T)$. We claim that $(\mathcal{V}, T, r)$ is a rooted tree decomposition. Suppose, for a contradiction, that there exist two nodes $t \in V(T) \backslash\{r\}$ and $t^{\prime} \in S_{T, r}(t)$ such that $V_{t} \cap V_{p(t)}=V_{t} \cap V_{t^{\prime}}$. Let $T^{\prime}$ be a tree such that $V\left(T^{\prime}\right)=V(T)$ and $E\left(T^{\prime}\right)=E(T) \backslash\left\{t t^{\prime}\right\} \cup\left\{p_{T, r}(t) t^{\prime}\right\}$. Next we show that $T^{\prime}$ is a full tree decomposition of $G$. For this, it suffices to show that $\left|V_{t^{\prime}} \cap V_{p_{T, r}(t)}\right|=$ $\operatorname{tw}(G)$. Let $p=p_{T, r}(t)$. As $V_{t} \cap V_{t^{\prime}}=V_{t} \cap V_{p}$, we have $V_{t} \cap V_{t^{\prime}}=V_{p} \cap V_{t} \cap V_{t^{\prime}}$. So, $\left|V_{t^{\prime}} \cap V_{p}\right| \geq\left|V_{t} \cap V_{t^{\prime}} \cap V_{p}\right|=\left|V_{t} \cap V_{t^{\prime}}\right|=\operatorname{tw}(G)$. As $\left|V_{t^{\prime}}\right|=\left|V_{p}\right|=\operatorname{tw}(G)+1$ and $V_{t^{\prime}} \neq V_{p}$, we have $\left|V_{t^{\prime}} \cap V_{p}\right|=\operatorname{tw}(G)$. Note that, for every $t^{\prime \prime} \neq t^{\prime}$ that is a descendant of $t^{\prime}$, we have $\left|P_{T^{\prime}, r}\left(t^{\prime \prime}\right)\right|=\left|P_{T, r}\left(t^{\prime \prime}\right)\right|-1$. Hence $\sum_{t \in V\left(T^{\prime}\right)}\left|P_{T^{\prime}, r}(t)\right|<\sum_{t \in V(T)}\left|P_{T, r}(t)\right|$, a contradiction to the choice of $(T, \mathcal{V}, r)$.

Given a rooted tree decomposition $(\mathcal{V}, T, r)$ of a graph $G$, and a node $t \in V(T) \backslash\{r\}$, we say that the vertex in $V_{t} \backslash V_{p(t)}$ is the representative of $t$. Observe that this vertex is indeed unique, because every rooted tree decomposition is also full.

\subsection{Partial 6-Trees}

In this section we prove Tuza's Conjecture on graphs with treewidth at most 6, also known as partial 6-trees (Proposition 2.2). A set of tools for dealing with graphs that contain vertices of small degree was introduced by Puleo [Pul15] (see Lemma 5.3), and 
Tuza's Conjecture was verified for graphs with maximum average degree less than 7 , i.e., for graphs in which every subgraph has average degree less than 7 . In this section, we extend the technique of Puleo (see Lemma 5.4) in order to prove Tuza's Conjecture for graphs with treewidth at most 6 (Theorem 5.6). The following example (Figure 5.2) shows that there are graphs with treewidth at most 6 whose maximum average degree is more than 7. So our result is not implied by the previous one.

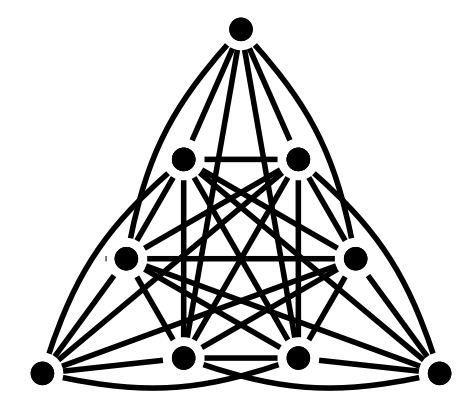

Figure 5.2: A graph with treewidth 6 (it is a 6-tree) and average degree $\frac{22}{3}>7$.

A nonempty set $V_{0} \subseteq V(G)$ is called reducible [Pul15, Definition 2.1] if there is a set $X \subseteq E(G)$ and a set $Y$ of edge-disjoint triangles in $G$ such that the following conditions hold:

(i) $|X| \leq 2|Y|$;

(ii) every triangle in $G$ containing a vertex of $V_{0}$ has an edge in $X$; and

(iii) if $u v \in E(A)$ for some $A \in Y$, and $u, v \notin V_{0}$, then $u v \in X$.

When $V_{0}, X$, and $Y$ satisfy the definition above, we say that $V_{0}$ is reducible using $X$ and $Y$. When $G$ has no reducible set, we say that $G$ is irreducible.

The following lemma comes naturally.

Lemma 5.2 ([uㅣㄹ, Lemma 2.2]). Let $G$ be a graph and $V_{0} \subseteq V(G)$ be reducible using $X$ and $Y$. Let $G^{\prime}=(G-X)-V_{0}$. If $\tau\left(G^{\prime}\right) \leq 2 v\left(G^{\prime}\right)$, then $\tau(G) \leq 2 v(G)$.

We say that a graph $G$ is robust [Pul15, Definition 2.3] if, for every $v \in V(G)$, every component of $G[N(v)]$ has at least 5 vertices. The following lemma (see [Pul15. Lemma 2.7]) is an important tool in our proofs. In what follows, the closed neighborhood $N(u) \cup\{u\}$ of a vertex $u \in V(G)$ is denoted by $N[u]$ and $\Delta(G)$ is the maximum degree of a vertex in $G$.

Lemma 5.3. If $G$ is an irreducible robust graph and $x, y \in V(G)$, then the following hold

(a) if $d(x) \leq 6$, then $\Delta(\overline{G[N(x)]}) \leq 1$ and $|E(\overline{G[N(x)]})| \neq 2$; 
(b) if $d(x) \leq 6$ and $d(y) \leq 6$, then $x y \notin E(G)$;

(c) if $d(x)=7$ and $d(y)=6$, then $N[y] \nsubseteq N[x]$;

(d) if $d(x) \leq 8$ and $d(y)=5$, then $N[y] \nsubseteq N[x]$.

In this section we extend the result above to the following lemma.

Lemma 5.4. If $G$ is an irreducible robust graph and $x, y \in V(G)$ are such that $d(x)$, $d(y) \leq 6$, and $|N(x) \cup N(y)| \leq 7$, then $d(x)=d(y)=5, \quad|N(x) \cap N(y)|=3$, and $G[N(x)], G[N(y)] \simeq K_{5}$.

Proof. Let $G, x$ and $y$ be as in the statement. Because $G$ is robust, $d(x), d(y) \geq 5$. We divide the proof on whether $d(x)=d(y)=6 ; d(x)=6$ and $d(y)=5$; or $d(x)=d(y)=5$.

Case 1: $d(x)=d(y)=6$.

Suppose, without loss of generality, that $N(x)=\left\{v_{1}, v_{2}, v_{3}, v_{4}, v_{5}, v_{6}\right\}$. By Lemma 5.3 a , $E(\overline{G[N(x)]})$ is either empty, an edge, or a matching of cardinality 3. Suppose, without loss of generality, that $E(\overline{G[N(x)]}) \subseteq\left\{v_{1} v_{4}, v_{2} v_{5}, v_{3} v_{6}\right\}$. If $N(y)=N(x)$, then put

$$
\begin{aligned}
& X=E\left(G\left[\left\{v_{1}, v_{2}, v_{3}, v_{4}, v_{5}, v_{6}\right\}\right]\right) \\
& Y=\left\{v_{1} v_{3} v_{5}, v_{2} v_{4} v_{6}, x v_{1} v_{2}, y v_{2} v_{3}, x v_{3} v_{4}, y v_{4} v_{5}, x v_{5} v_{6}, y v_{1} v_{6}\right\}
\end{aligned}
$$

Note that $|X| \leq 15 \leq 16=2|Y|$. It is not hard to check that $V_{0}=\{x, y\}$ is reducible using $X$ and $Y$, a contradiction.

Thus, we may assume that $|N(x) \cap N(y)|=5$. Suppose, without loss of generality, that $v_{1} \notin N(y)$. Thus, we may assume that $N(y)=\left\{v_{2}, v_{3}, v_{4}, v_{5}, v_{6}, v_{7}\right\}$, for some $v_{7} \in V(G)$. Since $E(\overline{G[N(x) \cap N(y)]}) \subseteq E(\overline{G[N(x)]})$, we have $E(\overline{G[N(x) \cap N(y)]}) \subseteq\left\{v_{2} v_{5}, v_{3} v_{6}\right\}$. If $E(\overline{G[N(x) \cap N(y)]})=\left\{v_{2} v_{5}, v_{3} v_{6}\right\}$, then $|E(G[N(x) \cap N(y)])|=8$. Now, let

$$
\begin{aligned}
& X=\left\{x v_{1}, y v_{7}\right\} \cup E\left(G\left[\left\{v_{2}, v_{3}, v_{4}, v_{5}, v_{6}\right\}\right]\right) \\
& Y=\left\{v_{2} v_{4} v_{6}, x v_{2} v_{3}, x v_{4} v_{5}, y v_{3} v_{4}, y v_{5} v_{6}\right\}
\end{aligned}
$$

Note that $|X|=10=2|Y|$. It is not hard to check that $V_{0}=\{x, y\}$ is reducible using $X$ and $Y$, a contradiction. Thus, we have $E(\overline{G[N(x) \cap N(y)]}) \leq 1$, and by Lemma 5.3 a), we have $|E(\overline{G[N(x)]})|,|E(\overline{G[N(y)]})| \leq 1$.

Suppose that $|E(\overline{G[N(x) \cap N(y)]})|=1$, then $|E(G[N(x) \cap N(y)])|=9$, and we may assume, without loss of generality, that $v_{3} v_{6} \in E(\overline{G[N(x) \cap N(y)]})$. This implies, by 
Lemma 5.3 a), that $v_{3} v_{7} \in E(G[N(y)])$. Now, let

$$
\begin{aligned}
& X=\left\{x v_{1}, y v_{7}, v_{3} v_{7}\right\} \cup E\left(G\left[\left\{v_{2}, v_{3}, v_{4}, v_{5}, v_{6}\right\}\right]\right) \\
& Y=\left\{v_{2} v_{4} v_{6}, v_{3} v_{4} v_{5}, x v_{2} v_{3}, x v_{5} v_{6}, y v_{2} v_{5}, y v_{3} v_{7}\right\}
\end{aligned}
$$

Note that $|X|=12=2|Y|$. It is not hard to check that $V_{0}=\{x, y\}$ is reducible using $X$ and $Y$, a contradiction (Figure 5.3(a)).

Thus, we may assume that $|E(\overline{G[N(x) \cap N(y)]})|=0$, i.e., $|E(G[N(x) \cap N(y)])|=10$. Now, let

$$
\begin{aligned}
& X=\left\{x v_{1}, y v_{7}\right\} \cup E\left(G\left[\left\{v_{2}, v_{3}, v_{4}, v_{5}, v_{6}\right\}\right]\right) \\
& Y=\left\{v_{2} v_{4} v_{6}, v_{3} v_{4} v_{5}, x v_{2} v_{3}, x v_{5} v_{6}, y v_{2} v_{5}, y v_{3} v_{6}\right\}
\end{aligned}
$$

Note that $|X|=12=2|Y|$. It is not hard to check that $V_{0}=\{x, y\}$ is reducible using $X$ and $Y$, a contradiction.

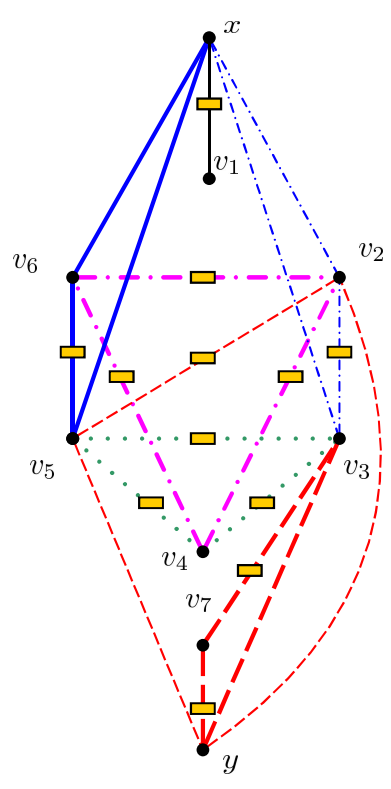

(a)

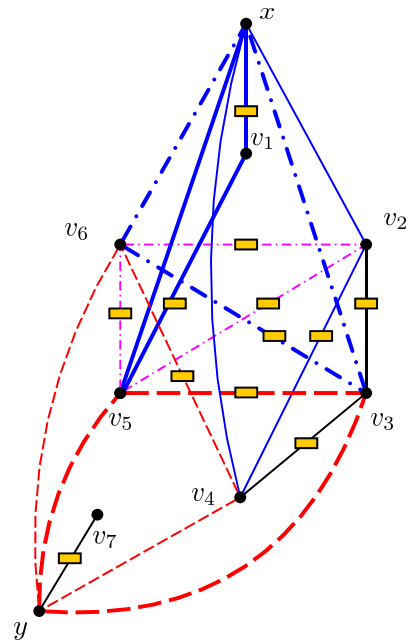

(b)

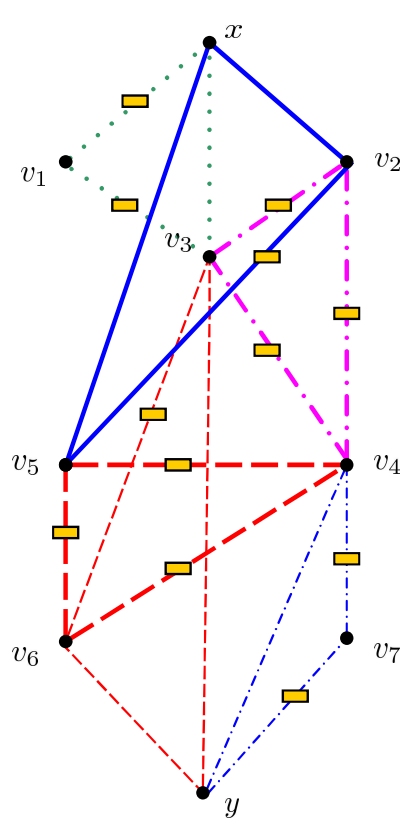

(c)

Figure 5.3: Cases in the proof of Lemma 5.4 In each figure, the corresponding packing is represented by triangles with different line traces, and the corresponding transversal is represented by the edges with little rectangles. (a) $d(x)=d(y)=6$ and $|E(\overline{G[N(x) \cap N(y)]})|=1 .(b) d(x)=6$,

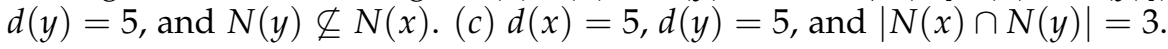

Case 2: $d(x)=6$ and $d(y)=5$.

First, suppose that $N(y) \subseteq N(x)$. Assume, without loss of generality, that $N(x)=$ $\left\{v_{1}, v_{2}, v_{3}, v_{4}, v_{5}, v_{6}\right\}$. By Lemma 5.3 a), $\overline{G[N(x)]}$ is either empty, or an edge, or a matching of cardinality 3 . Thus, we may assume, without loss of generality, that 
$E(\overline{G[N(x)]}) \subseteq\left\{v_{1} v_{4}, v_{2} v_{5}, v_{3} v_{6}\right\}$ and that $v_{1} \notin N(y)$, thus, $N(y)=\left\{v_{2}, v_{3}, v_{4}, v_{5}, v_{6}\right\}$. Analogously to the case above, we have that $E(\overline{G[N(x) \cap N(y)]}) \subseteq\left\{v_{2} v_{5}, v_{3} v_{6}\right\}$. Now, let

$$
\begin{aligned}
& X=\left\{x v_{1}, v_{1} v_{6}\right\} \cup E\left(G\left[\left\{v_{2}, v_{3}, v_{4}, v_{5}, v_{6}\right\}\right]\right) \\
& Y=\left\{v_{2} v_{4} v_{6}, x v_{1} v_{6}, x v_{4} v_{5}, x v_{2} v_{3}, y v_{3} v_{4}, y v_{5} v_{6}\right\}
\end{aligned}
$$

Note that $|X| \leq 12=2|Y|$. It is not hard to check that $V_{0}=\{x, y\}$ is reducible using $X$ and $Y$, a contradiction.

Thus, we may assume that $N(y) \nsubseteq N(x)$. In this case, $|N(x) \cap N(y)|=4$. Assume, without loss of generality, that $N(x)=\left\{v_{1}, v_{2}, v_{3}, v_{4}, v_{5}, v_{6}\right\}$, and that $N(y)=\left\{v_{3}, v_{4}, v_{5}, v_{6}, v_{7}\right\}$. By Lemma 5.3 a $),|E(\overline{G[N(x)]})|,|E(\overline{G[N(y)]})| \neq 2$ and $\Delta(\overline{G[N(x)]}), \Delta(\overline{G[N(y)]}) \leq 1$. Then, we may assume, without loss of generality, that either $E(\overline{G[N(x)]}) \cap E(\overline{G[N(y)]})=\left\{v_{4} v_{5}\right\}$ or $E(\overline{G[N(x)]}) \cap E(\overline{G[N(y)]})=\varnothing$. In the former, we may assume, without loss of generality, that $E(\overline{G[N(x)]}) \subseteq\left\{v_{1} v_{6}, v_{2} v_{3}, v_{4} v_{5}\right\}$ and $E(\overline{G[N(y)]})=\left\{v_{4} v_{5}\right\}$. In the latter, we may assume, without loss of generality, that $E(\overline{G[N(x)]}) \subseteq\left\{v_{1} v_{3}, v_{1} v_{4}\right\}$ and $E(\overline{G[N(y)]}) \subseteq\left\{v_{4} v_{7}, v_{5} v_{7}\right\}$. Let $H=G\left[\left\{v_{2}, v_{3}, v_{4}, v_{5}, v_{6}\right\}\right]$.

In the former case, we let

$$
\begin{aligned}
& X=\left\{x v_{1}, y v_{7}, v_{1} v_{5}\right\} \cup E(H) \\
& Y=\left\{x v_{1} v_{5}, x v_{2} v_{4}, x v_{3} v_{6}, y v_{3} v_{5}, y v_{4} v_{6}, v_{2} v_{5} v_{6}\right\}
\end{aligned}
$$

(Figure 5.3(b)). In the latter case, we let

$$
\begin{aligned}
X & =\left\{x v_{1}, y v_{7}\right\} \cup E(H) \\
Y & =\left\{x v_{3} v_{5}, x v_{4} v_{6}, y v_{3} v_{6}, y v_{4} v_{5}, v_{2} v_{3} v_{4}, v_{2} v_{5} v_{6}\right\}
\end{aligned}
$$

Note that, in both cases, $|X| \leq 12=2|Y|$. It is not hard to check that $V_{0}=\{x, y\}$ is reducible using $X$ and $Y$, a contradiction.

Case 3: $d(x)=d(y)=5$.

Assume, without loss of generality, that $N(x)=\left\{v_{1}, v_{2}, v_{3}, v_{4}, v_{5}\right\}$. In what follows, we divide the proof on whether $N(x) \cap N(y)$ has five, four, or three elements. Note that, by Lemma 5.3 a , $|E(G[N(x)])|,|E(G[N(y)])| \in\{9,10\}$.

First, suppose that $|N(x) \cap N(y)|=5$, which implies that $N(x)=N(y)$. We may assume, without loss of generality, that $E(\overline{G[N(x)]}) \subseteq\left\{v_{1} v_{2}\right\}$. Let

$$
\begin{aligned}
& X=E(G[N(x)]) \\
& Y=\left\{v_{1} v_{4} v_{5}, v_{2} v_{3} v_{5}, x v_{1} v_{3}, x v_{2} v_{4}, y v_{3} v_{4}\right\}
\end{aligned}
$$

Note that $|X| \leq 10=2|Y|$. It is not hard to check that $V_{0}=\{x, y\}$ is reducible using $X$ and $Y$, a contradiction. 
Now, suppose that $|N(x) \cap N(y)|=4$. Assume, without loss of generality, that $N(y)=\left\{v_{2}, v_{3}, v_{4}, v_{5}, v_{6}\right\}$, and let $H=G\left[\left\{v_{2}, v_{3}, v_{4}, v_{5}\right\}\right]$. Since $|E(G[N(x)])|,|E(G[N(y)])| \in\{9,10\}$, we may assume, without loss of generality, that $E(\bar{H}) \subseteq\left\{v_{3} v_{4}\right\}$. Let

$$
\begin{aligned}
& X=\left\{x v_{1}, y v_{6}\right\} \cup E(H) \\
& Y=\left\{x v_{2} v_{3}, x v_{4} v_{5}, y v_{2} v_{4}, y v_{3} v_{5}\right\} .
\end{aligned}
$$

Note that $|X| \leq 8=2|Y|$. It is not hard to check that $V_{0}$ is reducible using $X$ and $Y$, a contradiction.

Finally, suppose that $|N(x) \cap N(y)|=3$. Assume, without loss of generality that $N(y)=\left\{v_{3}, v_{4}, v_{5}, v_{6}, v_{7}\right\}$, and let $H=G\left[\left\{v_{3}, v_{4}, v_{5}\right\}\right]$. Suppose that $|E(H)|<3$. Then we may assume that $v_{3} v_{5} \notin E(H)$ and $v_{3} v_{4}, v_{4} v_{5} \in E(H)$. Let

$$
\begin{aligned}
X & =\left\{v_{2} v_{3}, v_{3} v_{4}, v_{2} v_{4}, v_{4} v_{5}, v_{5} v_{6}, v_{6} v_{4}, v_{2} v_{5}, v_{3} v_{6}, v_{1} v_{3}, v_{7} v_{4}, x v_{1}, y v_{7}\right\} \\
Y & =\left\{v_{2} v_{3} v_{4}, v_{4} v_{5} v_{6}, x v_{2} v_{5}, y v_{3} v_{6}, x v_{1} v_{3}, y v_{7} v_{4}\right\} .
\end{aligned}
$$

Note that $|X|=12=2|Y|$. It is not hard to check that $V_{0}=\{x, y\}$ is reducible using $X$ and $Y$, a contradiction (Figure 5.3(c)). We conclude that the set $N(x) \cap N(y)$ induces a triangle in $G$.

Suppose by contradiction that either $G[N(x)]$ or $G[N(y)]$, without loss of generality $G[N(y)]$, does not induce a complete graph. Since $N(x) \cap N(y)$ induces a triangle in $G$, we may assume, without loss of generality, that $E(\overline{G[N(y)]}) \subseteq\left\{v_{3} v_{6}, v_{6} v_{7}\right\}$. Let $v_{x}$ be a neighbor of $v_{1}$ in $\left\{v_{2}, v_{3}\right\}$. Let $v_{y}$ be a neighbor of $v_{6}$ in $\left\{v_{3}, v_{7}\right\}$. Then let

$$
\begin{aligned}
X & =E(G[N(y)]) \cup\left\{x v_{1}, x v_{2}, v_{1} v_{x}\right\} \\
Y & =\left\{v_{3} v_{4} v_{7}, v_{y} v_{5} v_{6}, x v_{1} v_{x}, x v_{4} v_{5}, y v_{5} v_{7}, y v_{4} v_{6}\right\} .
\end{aligned}
$$

Note that $|X|=12=2|Y|$. It is not hard to check that $V_{0}=\{x, y\}$ is reducible using $X$ and $Y$, a contradiction.

This concludes the proof.

Recall that, given a rooted tree decomposition $(T, \mathcal{V}, r)$ of a graph $G$, the representative of a node $t \neq r$ is the (unique) vertex in $V_{t} \backslash V_{p(t)}$.

Proposition 5.5. Given a rooted tree decomposition $(T, \mathcal{V}, r)$ of a graph $G$, if $t \neq r$ is a leaf in $T$ and $y$ is the representative of $t$, then $N(y) \subseteq V_{t}$.

Proof. Suppose that there is a vertex $y^{\prime} \in N(y) \backslash V_{t}$. By condition (T2) of the definition of tree decomposition, there is a bag $V_{t^{\prime}}$ such that $y, y^{\prime} \in V_{t^{\prime}}$, and hence, by condition (T3) of the definition of tree decomposition, as $t$ is a leaf, we have $y \in V_{p(t)}$, a contradiction. 
The following theorem is the main result of this section.

Theorem 5.6. If $G$ is a graph with treewidth at most 6 , then $\tau(G) \leq 2 v(G)$.

Proof. Suppose, for a contradiction, that the statement does not hold, and let $G$ be a graph with treewidth at most $6, \tau(G)>2 v(G)$, that minimizes $|V(G)|$. We claim that $G$ is irreducible. Indeed, suppose that there exists a set $V_{0} \subseteq V(G)$ that is reducible using $X$ and $Y$. Note that $G^{\prime}$ has treewidth at most 6 and, by the minimality of $G$, we have that $\tau\left(G^{\prime}\right) \leq 2 v\left(G^{\prime}\right)$. Thus, by Lemma 5.2, we have $\tau(G) \leq 2 v(G)$, a contradiction.

Claim 5.7. $G$ is robust.

Proof. Suppose, for a contradiction, that $x$ is a vertex of $G$ such that $G[N(x)]$ contains a component $C$ of cardinality at most 4 . Let $E_{C}=\{x v: v \in V(C)\}$. Let $M^{\prime}$ be a maximum matching in $C$. If $M^{\prime}=\varnothing$, then there is no triangle in $G$ containing the edges in $E_{C}$. Therefore, every transversal of $G^{\prime}=G-E_{C}$ is a transversal of $G$, i.e., $\tau(G) \leq \tau\left(G^{\prime}\right)$. Moreover, every triangle packing of $G^{\prime}$ is a triangle packing of $G$, which implies $v(G) \geq v\left(G^{\prime}\right)$. By the minimality of $G$, we have $\tau\left(G^{\prime}\right) \leq 2 v\left(G^{\prime}\right)$, and hence $\tau(G) \leq 2 v(G)$, a contradiction. If $M^{\prime}=\left\{v_{1} v_{2}\right\}$, then $C$ is either a star or a triangle. If $C$ is a star, let $u$ be the center of $C$, and if $C$ is a triangle, then let $u$ be the vertex of $C$ not incident to the edge in $v_{1} v_{2}$. Note that if $A$ is a triangle of $G$ containing edges of $E_{C}$, then $A$ contains $v_{1} v_{2}$ or $A$ contains $x u$. Let $G^{\prime}=G-E\left(x v_{1} v_{2}\right)$. By the minimality of $G$, we have $\tau\left(G^{\prime}\right) \leq 2 v\left(G^{\prime}\right)$. Let $X^{\prime}$ and $Y^{\prime}$ be a minimum transversal and a maximum packing of $G^{\prime}$, respectively. Note that $X^{\prime} \cup\left\{v_{1} v_{2}, x u\right\}$ is a transversal of $G$, and $Y^{\prime} \cup\left\{x v_{1} v_{2}\right\}$ is a packing of $G$. Hence

$$
\tau(G) \leq\left|X^{\prime}\right|+2=\tau\left(G^{\prime}\right)+2 \leq 2 v\left(G^{\prime}\right)+2=2\left|Y^{\prime}\right|+2 \leq 2 v(G),
$$

a contradiction.

If $M^{\prime}=\left\{u_{1} u_{2}, v_{1} v_{2}\right\}$, then we put $G^{\prime}=G-E\left(x u_{1} u_{2}\right)-E\left(x v_{1} v_{2}\right)$. By the minimality of $G$, we have $\tau\left(G^{\prime}\right) \leq 2 v\left(G^{\prime}\right)$. Let $X^{\prime}$ and $Y^{\prime}$ be a minimum transversal and a maximum packing of $G^{\prime}$, respectively. Note that $X^{\prime} \cup\left\{u_{1} u_{2}, v_{1} v_{2}, x v_{1}, x v_{2}\right\}$ is a transversal of $G$, and $Y^{\prime} \cup\left\{x u_{1} u_{2}, x v_{1} v_{2}\right\}$ is a packing of $G$. Hence

$$
\tau(G) \leq\left|X^{\prime}\right|+4=\tau\left(G^{\prime}\right)+4 \leq 2 v\left(G^{\prime}\right)+4=2\left|Y^{\prime}\right|+4 \leq 2 v(G),
$$

a contradiction.

Suppose that $|V(G)| \leq 7$ and let $x, y \in V(G)$. Since $G$ is irreducible and robust, and $d(x), d(y) \leq 6$, by Lemma 5.3 b), we have $|E(G)|=\varnothing$. Thus $\tau(G) \leq 2 v(G)$, a contradiction. Therefore, we may assume $|V(G)| \geq 8$. By Proposition 5.1, $G$ has a rooted tree decomposition $(T, \mathcal{V}, r)$ of width $\operatorname{tw}(G) \leq 6$. Since $|V(G)| \geq 8$, we 
have $|V(T)|>1$, and hence there is a node $t \in V(T)$ with $h(t)=1$. If $t \neq r$, then let $v_{t}$ be the representative of $t$; otherwise, let $v_{t}$ be an arbitrary vertex of $V_{t}$. First, suppose that $S(t)=\left\{t^{\prime}\right\}$. Let $x$ be the representative of $t^{\prime}$. By Proposition 5.5, we have that $d(x) \leq\left|V_{t^{\prime}}\right|-1 \leq 6$, and, by Claim 5.7, we have $d(x) \in\{5,6\}$. Also, by Proposition 5.5 applied to $G-x,\left(T-t^{\prime}, \mathcal{V} \backslash\left\{V_{t^{\prime}}\right\}, r\right)$, and $t$, we have that $N_{G-x}\left(v_{t}\right) \subseteq V_{t}$ (if $t=r$, then this holds trivially). Thus $d\left(v_{t}\right)=\left|N\left(v_{t}\right)\right| \leq\left|N_{G-x}\left(v_{t}\right)\right|+1 \leq\left|V_{t}\right| \leq 7$.

Note that if $d\left(v_{t}\right)=7$, then $v_{t}$ must be incident to every vertex in $V_{t} \backslash\left\{v_{t}\right\}$, and also to $x$. Since $V_{t^{\prime}} \subseteq V_{t} \cup\{x\}$, we have $N[x] \subseteq N\left[v_{t}\right]$, a contradiction either to Lemma 5.3(c) or to Lemma 5.3 d). Therefore, we may assume that $d\left(v_{t}\right) \leq 6$, and hence, by Lemma $5.3(\mathrm{~b})$, we have $v_{t} x \notin E(G)$. Thus, we have $N(x) \cup N\left(v_{t}\right) \subseteq V_{t} \backslash\left\{v_{t}\right\}$, which implies $\left|N(x) \cup N\left(v_{t}\right)\right| \leq\left|V_{t}\right|-1 \leq 6$. On the other hand, by Lemma 5.4, we have $d\left(v_{t}\right)=d(x)=5$ and $\left|N(x) \cap N\left(v_{t}\right)\right|=3$, which implies $\left|N(x) \cup N\left(v_{t}\right)\right|=7$, a contradiction.

Therefore we may assume that $|S(t)|>1$. First, suppose that $|S(t)| \geq 3$, and let $t_{1}, t_{2}, t_{3} \in S(t)$. Let $z_{i}$ be the representative of $t_{i}$, for $i=1,2,3$. Note that, by Proposition 5.5. $\left|N\left(z_{i}\right) \cup N\left(z_{j}\right)\right| \leq\left|V_{t}\right|=7$, for $i, j \in\{1,2,3\}$ and $i \neq j$. Thus, by Lemma 5.4. $d\left(z_{i}\right)=5$, and $G\left[N\left(z_{i}\right)\right] \simeq K_{5}$ for every $i \in\{1,2,3\}$, and $\left|N\left(z_{i}\right) \cap N\left(z_{j}\right)\right|=3$, for $i, j \in\{1,2,3\}$ and $i \neq j$. Let $N\left(z_{1}\right)=\left\{v_{1}, v_{2}, v_{3}, v_{4}, v_{5}\right\}$. Since $\left|N\left(z_{1}\right) \cap N\left(z_{2}\right)\right|=3$, we may assume, without loss of generality, that $N\left(z_{2}\right)=\left\{v_{3}, v_{4}, v_{5}, v_{6}, v_{7}\right\}$. It is not hard to check that, since $\left|N\left(z_{1}\right) \cap N\left(z_{3}\right)\right|=\left|N\left(z_{2}\right) \cap N\left(z_{3}\right)\right|=3$ and $\left|N\left(z_{3}\right)\right|=5$, then $N\left(z_{3}\right)$ contains exactly one vertex in $N\left(z_{1}\right) \cap N\left(z_{2}\right)$. So we may assume, without loss of generality, that $N\left(z_{3}\right)=\left\{v_{1}, v_{2}, v_{3}, v_{6}, v_{7}\right\}$. Let $H=G\left[\left\{v_{1}, v_{2}, v_{3}, v_{4}, v_{5}, v_{6}, v_{7}\right\}\right]$ and note that every pair of vertices of $H$ is contained in at least one $N\left(z_{i}\right)$ for $i \in\{1,2,3\}$. Thus, since $G\left[N\left(z_{i}\right)\right] \simeq K_{5}$ for $i \in\{1,2,3\}$, we have $H \simeq K_{7}$. Let $X=E(H)$ and note that $|X|=21$. Let

$$
\begin{aligned}
& Y_{1}=\left\{z_{1} v_{1} v_{3}, z_{1} v_{2} v_{5}, z_{2} v_{3} v_{4}, z_{2} v_{5} v_{6}, z_{3} v_{1} v_{6}, z_{3} v_{2} v_{4}\right\} \\
& Y_{2}=\left\{v_{1} v_{2} v_{7}, v_{2} v_{3} v_{6}, v_{4} v_{6} v_{7}, v_{3} v_{5} v_{7}, v_{1} v_{4} v_{5}\right\}
\end{aligned}
$$

and note that for $Y=Y_{1} \cup Y_{2}$, we have $|Y|=11$ and $|X| \leq 2|Y|$. Hence $V_{0}=\left\{z_{1}, z_{2}, z_{3}\right\}$ is reducible using $X$ and $Y$, a contradiction (Figure 5.4). We conclude that $|S(t)| \leq 2$.

Recall that, when $t \neq r, v_{t}$ is the representative of $t$ and, if $t=r$, then $v_{t}$ is an arbitrary vertex of $V_{t}$. Let $t_{1}, t_{2} \in S(t)$ and let $x, y$ be the representatives of $t_{1}, t_{2}$, respectively. Again, by Lemma 5.4, we have $d(x)=d(y)=5,|N(x) \cap N(y)|=3$, and $G[N(x)], G[N(y)] \simeq K_{5}$. This implies that $V_{t} \subseteq N(x) \cup N(y)$. Note that $t$ is a leaf of $\left(T^{\prime}, \mathcal{V}^{\prime}, r\right)$, where $T^{\prime}=T-t_{1}-t_{2}$ and $\mathcal{V}^{\prime}=\mathcal{V} \backslash\left\{V_{t_{1}}, V_{t_{2}}\right\}$, hence $d_{G-x-y}\left(v_{t}\right) \leq 6$. Thus, we have $d_{G}\left(v_{t}\right) \leq 8$. Note that $v_{t} \in N(x) \cup N(y)$ and assume, without loss of generality, that $v_{t} \in N(x)$. Since $G[N(x)]$ is a complete graph, we have $N[x] \subseteq N\left[v_{t}\right]$, a contradiction to Lemma 5.3 dd). This concludes the proof. 


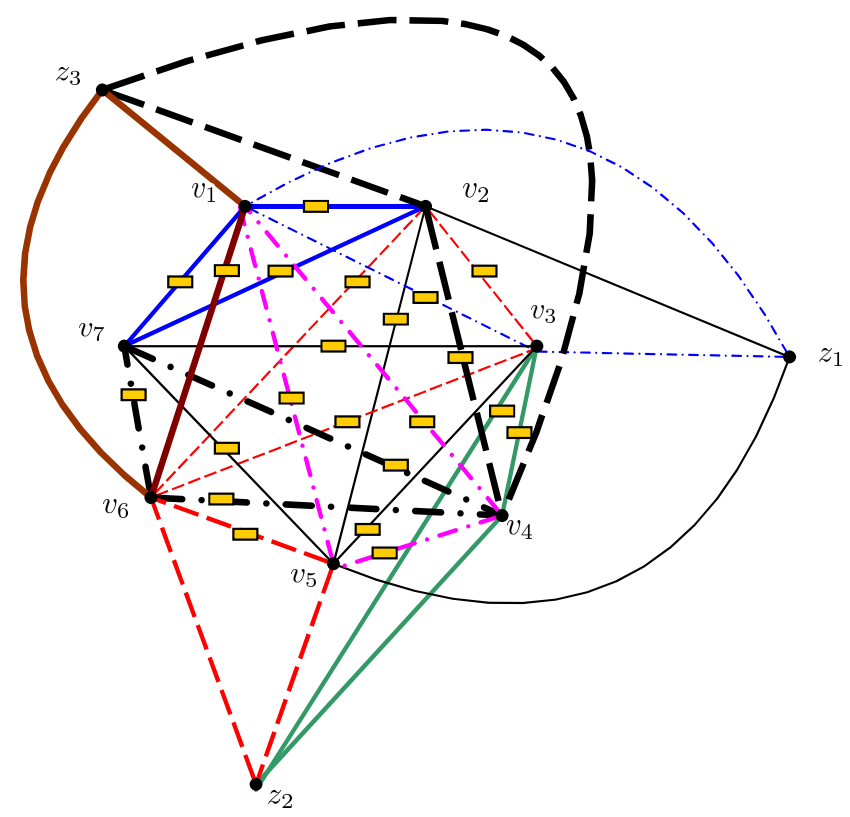

Figure 5.4: A case in the proof of Theorem 5.6. The corresponding packing is represented by triangles with different line traces, and the corresponding transversal is represented by the edges with small rectangles.

To finish this section, we mention that Theorem 5.6 generalizes a result of Tuza about chordal graphs [Tuz90, Proposition 3].

Corollary 5.8. If $G$ is a $K_{8}$-free chordal graph, then $\tau(G) \leq 2 v(G)$.

Proof. As $G$ is $K_{8}$-free, we have $\omega(G) \leq 7$. Thus, by Proposition 2.3, we have $\operatorname{tw}(G)=\omega(G)-1 \leq 6$, and $\tau(G) \leq 2 v(G)$ by Theorem 5.6 .

\subsection{3-Trees}

It is easy to see, by Theorem 5.6, that Tuza's Conjecture holds for 3-trees, as they have treewidth at most 6 (Proposition 2.4). In this section, we improve this result. We prove that $\tau(G) \leq \frac{9}{5} v(G)+\frac{1}{5}$ if $G$ is a 3-tree (Theorem 5.10). We say that a pair $(X, Y)$ is a $\frac{9}{5}$-TP of a graph $G$ if $X$ is a transversal of $G, Y$ is a packing of $G$, and $|X| \leq \frac{9}{5} \cdot|Y|+\frac{1}{5}$. Observe that if $G$ has a $\frac{9}{5}$-TP, then $\tau(G) \leq \frac{9}{5} v(G)+\frac{1}{5}$.

Let $(T, \mathcal{V}, r)$ be a rooted tree decomposition of a graph $G$. Given a node $t \in V(T) \backslash\{r\}$, we denote by $R(t)$ the set of all representatives of all descendants of $t$. Recall that $S(t)$ is the set of successors of $t$. For every triple of vertices $\Delta \subseteq V_{t}$, we let $S^{\Delta}(t)=\left\{t^{\prime} \in S(t): V_{t^{\prime}} \cap V_{t}=\Delta\right\}$. When $t$ is clear from the context, we simply write $S^{\Delta}$.

Our proof strategy is to analyze nodes in the tree decomposition with small height. 
We start by analyzing nodes with height one and conclude that a minimal counterexample to our theorem must have a particular configuration around such nodes.

Lemma 5.9. Let $G$ be a 3-tree such that $\tau(G)>\frac{9}{5} \nu(G)+\frac{1}{5}$, and $G$ is minimal over all such graphs. If $(T, \mathcal{V}, r)$ is a rooted tree decomposition of $G$ of width $3, t \in V(T) \backslash\{r\}$, and $h(t)=1$, then $|S(t)|=1$.

Proof. Let $V_{t}=\{a, b, c, d\}$. Suppose by contradiction that $|S(t)|>1$. Without loss of generality, assume that $V_{p(t)} \cap V_{t}=\{b, c, d\}$. Let $S(t)=\left\{t_{1}, t_{2}, \ldots, t_{k}\right\}$. For every $i \in[k]$, let $v_{i}$ be the representative of $t_{i}$. As $h(t)=1$, at least one of $\Delta$ in $\{a b c, a b d, a c d\}$ is such that $S^{\Delta} \neq \varnothing$.

Case 1: Exactly one triangle $\Delta$ in $\{a b c, a b d, a c d\}$ is such that $S^{\Delta} \neq \varnothing$.

Without loss of generality, let $S^{a b c} \neq \varnothing$. Let $G^{\prime}=G-R(t)$. Note that $G^{\prime}$ is a 3-tree. As $G$ is minimal, there exists a $\frac{9}{5}$-TP of $G^{\prime}$, say $\left(X^{\prime}, Y^{\prime}\right)$. Thus, $\left(X^{\prime} \cup\{a b, b c, a c\}, Y^{\prime} \cup\right.$ $\left.\left\{a c v_{1}, a b v_{2}\right\}\right)$ is a $\frac{9}{5}$-TP of $G$, a contradiction. Indeed, $\tau(G) \leq \tau\left(G^{\prime}\right)+3 \leq \frac{9}{5} v\left(G^{\prime}\right)+\frac{16}{5} \leq$ $\frac{9}{5}(v(G)-2)+\frac{16}{5} \leq \frac{9}{5} v(G)+\frac{1}{5}$ (Figure 5.5(a)).

Case 2: At least two triangles $\Delta$ in $\{a b c, a b d, a c d\}$ are such that $S^{\Delta} \neq \varnothing$.

Case 2.1: $|S(t)|=2$.

Without loss of generality, assume that $t_{1} \in S^{a b c}$ and $t_{2} \in S^{a b d}$. Let $G^{\prime}=G-R(t)$. Note that $G^{\prime}$ is a 3-tree. As $G$ is minimal, there exists a $\frac{9}{5}-\mathrm{TP}$ of $G^{\prime}$, say $\left(X^{\prime}, Y^{\prime}\right)$. Let $e \in X^{\prime} \cap E(b c d)$. Without loss of generality, we have two cases. If $e=b c$, then we let $X=X^{\prime} \cup\left\{a d, a v_{1}, b v_{2}\right\}$. If $e=c d$, then we let $X=X^{\prime} \cup\left\{a b, c v_{1}, d v_{2}\right\}$. In any case, $\left(X, Y^{\prime} \cup\left\{a c v_{1}, a b v_{2}\right\}\right)$ is a $\frac{9}{5}$-TP of $G$, a contradiction (Figure 5.5(b)).

Case 2.2: $|S(t)| \geq 3$.

Without loss of generality, assume that either $t_{1}, t_{2} \in S^{a b c}$ and $t_{3} \in S^{a b d}$, or $t_{1} \in S^{a b c}, t_{2} \in S^{a b d}$ and $t_{3} \in S^{a c d}$. Let $G^{\prime}=G-R(t)$. As $G$ is minimal, there exists a $\frac{9}{5}$-TP of $G^{\prime}$, say $\left(X^{\prime}, Y^{\prime}\right)$. Without loss of generality suppose that $c d \in X^{\prime}$. Then $\left(X^{\prime} \cup\{a b, b c, a c, b d, a d\}, Y^{\prime} \cup\left\{a c v_{1}, a b v_{2} a d v_{3}\right\}\right)$ is a $\frac{9}{5}$-TP of $G$, a contradiction (Figure 5.5(c)). 


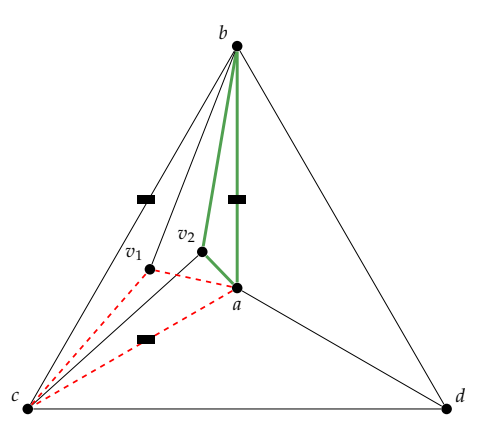

(a)

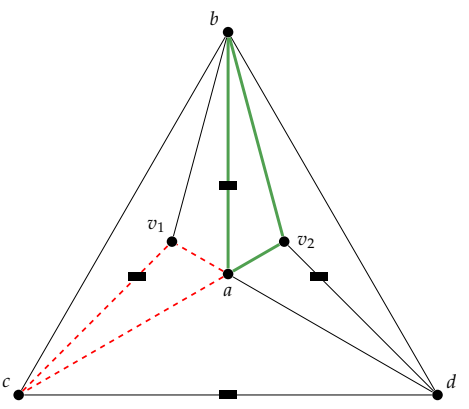

(b)

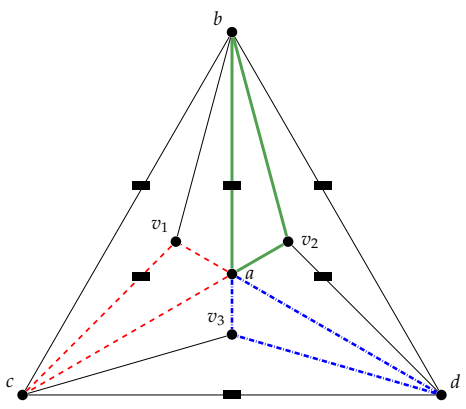

(c)

Figure 5.5: Situations in the proof of Lemma 5.9

This concludes the proof of the Lemma.

Finally, we prove the main theorem of this section.

Theorem 5.10. If $G$ is a 3-tree, then $\tau(G) \leq \frac{9}{5} v(G)+\frac{1}{5}$.

Proof. Suppose by contradiction that the statement does not hold. Let $G$ be a minimal counterexample over all 3-trees with $\tau(G)>\frac{9}{5} v(G)+\frac{1}{5}$.

Claim 5.11. If $(T, \mathcal{V}, r)$ is a rooted tree decomposition of $G$ of width three, then $|V(T)| \geq 4$.

Proof. If $|V(T)|=1$, then $|V(G)|=4, \tau(G)=2$, and $v(G)=1$, a contradiction. If $|V(T)|=2$, then $|V(G)|=5, \tau(G)=3$, and $v(G)=2$, a contradiction. If $|V(T)|=3$, then $|V(G)|=6, \tau(G) \leq 4$, and $v(G)=3$, again a contradiction.

Claim 5.12. If $(T, \mathcal{V}, r)$ is a rooted tree decomposition of $G$ of width three, then $T$ is not a star.

Proof. Suppose by contradiction that $T$ is a star. Suppose for a moment that $T$ is centered at $r$. Then $h(r) \leq 1$. By Claim $5.11|V(T)| \geq 4$, so $h(r) \geq 1$. Hence, $h(r)=1$ and we have two cases. First suppose that, for every $p, q \in S(r), V_{p} \cap V_{r}=V_{q} \cap V_{r}$. Without loss of generality, let $V_{r}=\{a, b, c, d\}$ and $V_{r} \cap V_{p}=\{a, b, c\}$ for every $p \in S(r)$. Let $p \in S(r)$ and let $v$ be the representative of $p$. Then $\left(E(a b c),\left\{a b d, a c v_{1}\right\}\right)$ is a $\frac{9}{5}$-TP of $G$, a contradiction.

Now suppose that there exist two nodes $p, q \in S(r)$ with $V_{p} \cap V_{r} \neq V_{q} \cap V_{r}$. We may assume that $p$ is a successor of $r$ such that $\left|\left\{t^{\prime} \in S_{T, r}(r): V_{p} \cap V_{r}=V_{t^{\prime}} \cap V_{r}\right\}\right|$ is minimum. Let $A=\left\{t^{\prime} \in S_{T, r}(r) \backslash\{p\}: V_{p} \cap V_{r}=V_{t^{\prime}} \cap V_{r}\right\}$. Let $T^{\prime}$ be a tree with $V\left(T^{\prime}\right)=V(T)$ and

$$
E\left(T^{\prime}\right)=E(T) \backslash\left\{r t^{\prime}: t^{\prime} \in A\right\} \cup\left\{p t^{\prime}: t^{\prime} \in A\right\} .
$$


Then the triple $\left(T^{\prime}, \mathcal{V}, p\right)$ is a rooted tree decomposition of $G$. By Lemma 5.9. $\left|S_{T^{\prime}, p}(r)\right|=1$. Note that $S_{T^{\prime}, p}(r)=S_{T, r}(r) \backslash(\{p\} \cup A)$. By the choice of $p,|A|=0$. Indeed, suppose for a moment that $|A|>0$. Let $q$ be the only element of $S_{T^{\prime}, p}(r)$. Then $\left|\left\{t^{\prime} \in S_{T, r}(r): V_{q} \cap V_{r}=V_{t^{\prime}} \cap V_{r}\right\}\right|=\mid\left\{t^{\prime} \in S_{T^{\prime}, p}(r): V_{q} \cap V_{r}=\right.$ $\left.V_{t^{\prime}} \cap V_{r}\right\} \mid=1$, a contradiction to the choice of $p$. This implies that $|V(T)|=3$, a contradiction.

Hence, $T$ is not centered at $r$, which implies that $|S(r)|=1$. So $r_{1}$, the only successor of $r$, is the center of $T$, which implies that $h\left(r_{1}\right)=1$. Hence $\left|S\left(r_{1}\right)\right|=1$ by Lemma 5.9 . Then $|V(T)|=3$, a contradiction to Claim 5.11 .

Claim 5.13. There exists a rooted tree decomposition $(T, \mathcal{V}, r)$ of $G$ of width three with a node $t \in V(T) \backslash\{r\}$ such that $h(t)=2$.

Proof. Note that $|V(G)| \geq 4$. So, by Proposition 2.4. $G$ has treewidth three. Thus, by Proposition 5.1. $G$ has a rooted tree decomposition, say $(T, \mathcal{V}, r)$, of width 3 . If $h_{T, r}(r) \geq 3$, then we are done. If $h_{T, r}(r) \leq 1$, then $T$ is a star centered at $r$, a contradiction to Claim 5.12. So, let us assume that $h_{T, r}(r)=2$, which implies that there exists a path $r r_{1} r_{2}$ in $T$ with $h_{T, r}\left(r_{1}\right)=1$ and, by Lemma 5.9. $S_{T, r}\left(r_{1}\right)=\left\{r_{2}\right\}$. If $\left|S_{T, r}(r)\right|=1$, then $T$ is a star centered at $r_{1}$, a contradiction to Claim 5.12, So, let us assume that $\left|S_{T, r}(r)\right|>1$.

Let $\bar{S}(r)=\left\{t^{\prime} \in S_{T, r}(r) \backslash\left\{r_{1}\right\}: V_{r} \cap V_{t^{\prime}}=V_{r} \cap V_{r_{1}}\right\}$. Let $T^{\prime}$ be a tree with $V\left(T^{\prime}\right)=V(T)$ and

$$
E\left(T^{\prime}\right)=E(T) \backslash\left\{r t^{\prime}: t^{\prime} \in \bar{S}(r)\right\} \cup\left\{r_{1} t^{\prime}: t^{\prime} \in \bar{S}(r)\right\}
$$

Then the triple $\left(T^{\prime}, \mathcal{V}, r_{2}\right)$ is a rooted tree decomposition of $G$ of width 3. If $h_{T^{\prime}, r_{2}}\left(r_{1}\right) \geq 2$, then we are done. Thus $h_{T^{\prime}, r_{2}}\left(r_{1}\right)=1$, which implies, by Lemma 5.9 , that $S_{T^{\prime}, r_{2}}\left(r_{1}\right)=\{r\}$ and $|V(T)|=3$, a contradiction to Claim 5.11.

Let $(T, \mathcal{V}, r)$ be a rooted tree decomposition of $G$ given by Claim 5.13, and let $t$ be the node in $T$ with $t \neq r$ and $h(t)=2$. Let $L=\left\{\ell_{1}, \ell_{2}, \ldots, \ell_{m}\right\}$ be the set of successors of $t$ that are leaves, that is, $h\left(\ell_{i}\right)=0$ for every $i \in[m]$. For every $i \in[m]$, let $u_{i}$ be the representative of $\ell_{i}$. Let $Q=S(t) \backslash L=\left\{q_{1}, q_{2}, \ldots, q_{k}\right\}$. That is, $h\left(q_{i}\right)=1$ for every $i \in[k]$. By Lemma 5.9, $\left|S\left(q_{i}\right)\right|=1$ for every $i \in[k]$. For every such $i$, let $S\left(q_{i}\right)=\left\{q_{i}^{\prime}\right\}$. Also, let $Q^{\prime}=\left\{q_{1}^{\prime}, q_{2}^{\prime}, \ldots, q_{k}^{\prime}\right\}$. For every $i \in[k]$, let $v_{i}$ be the representative of $q_{i}$ and let $v_{i}^{\prime}$ be the representative of $q_{i}^{\prime}$. Also, as $h(t)=2$, we must have $k \geq 1$ (Figure 5.6).

Without loss of generality let $V_{t}=\{a, b, c, d\}$ and $V_{t} \cap V_{p(t)}=\{b, c, d\}$.

Claim 5.14. Let $G^{\prime}=G-R(t)$. If $X^{\prime}$ is a transversal of $G^{\prime}$, then there exists a transversal of $G$ with cardinality $\left|X^{\prime}\right|+1+m+2 k$. 


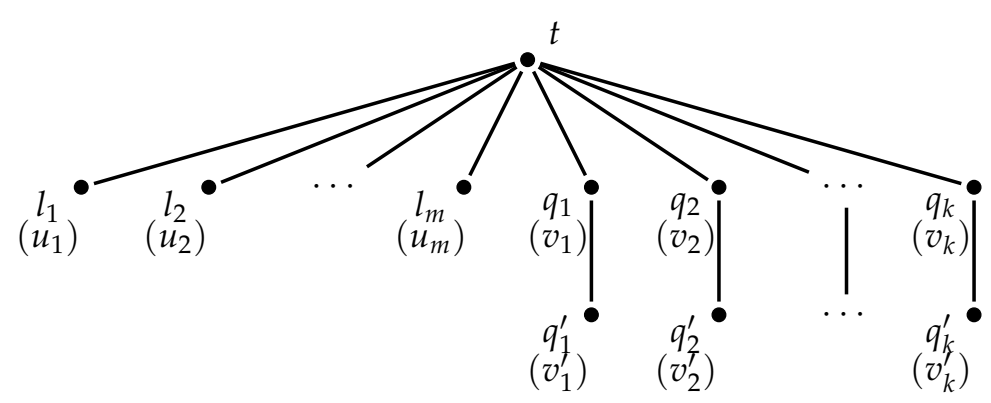

Figure 5.6: Situation in the proof of Theorem 5.10

Proof. As $X^{\prime}$ is a transversal of $G^{\prime}$ and $b c d$ is a triangle in $G^{\prime}, X^{\prime} \cap E(b c d) \neq \varnothing$. Without loss of generality, we may assume that $b c \in X^{\prime}$. Given a 4-clique $K$ in $G$ and a set $X \subseteq E(G)$ such that $E(K) \cap X=\{e\}$, it is denoted by $K \otimes X$ the only edge in $K$ that does not share any vertex with $e$. For instance, $V_{t} \otimes\{b c\}=a d$.

For every $i \in[m]$, we let $e_{i}=V_{\ell_{i}} \otimes\{b c, a d\}$. For every $i \in[k]$, we let $f_{i}=V_{q_{i}} \otimes\{b c, a d\}$ and $f_{i}^{\prime}=V_{q_{i}^{\prime}} \otimes\left\{b c, a d, f_{i}\right\}$. Note that $X=X^{\prime} \cup\{a d\} \cup\left\{e_{i}: i \in[m]\right\} \cup$ $\left\{f_{i}: i \in[k]\right\} \cup\left\{f_{i}^{\prime}: i \in[k]\right\}$ is a transversal of $G$ and $|X|=\left|X^{\prime}\right|+1+m+2 k$.

Claim 5.15. Let $G^{\prime}=G-R(t)$. If $X^{\prime}$ is a transversal of $G^{\prime}$, then there exists a transversal of $G$ of cardinality at most $\left|X^{\prime}\right|+5+k$.

Proof. Let $X=X^{\prime} \cup E\left(G\left[V_{t}\right]\right) \cup\left\{v_{i} v_{i}^{\prime}: i \in[k]\right\}$. Note that $X$ is a transversal of $G$. As $X^{\prime}$ is a transversal of $G^{\prime}$ and $b c d$ is a triangle in $G^{\prime}, X^{\prime} \cap E(b c d) \neq \varnothing$ so $\left|X^{\prime} \cap E\left(G\left[V_{t}\right]\right)\right| \geq 1$. Thus $|X|=\left|X^{\prime}\right|+\left|E\left(G\left[V_{t}\right]\right)\right|-\left|X^{\prime} \cap E\left(G\left[V_{t}\right]\right)\right|+k \leq\left|X^{\prime}\right|+5+k$.

Claim 5.16. Let $\Delta$ be a triple of vertices in $V_{t}$ with $\Delta \neq V_{t} \cap V_{p(t)}$. Let $t^{\prime} \in S^{\Delta}(t)$. Let $G^{\prime}=G-R\left(t^{\prime}\right)$. If $\left|S^{\Delta}(t)\right| \geq 3$, then there exists a minimum transversal $X^{\prime}$ of $G^{\prime}$ with $E(\Delta) \subseteq X^{\prime}$.

Proof. Without loss of generality, let $\Delta=a b d$. We may also assume that $S^{\Delta}(t) \backslash\left\{t^{\prime}\right\}=\left\{\ell_{1}, \ell_{2}, \ldots, \ell_{m^{\prime}}\right\} \cup\left\{q_{1}, q_{2}, \ldots, q_{k^{\prime}}\right\}$. Let $X^{\prime}$ be a minimum transversal of $G^{\prime}$. For every $i \in\left[m^{\prime}\right]$, let $F_{i}=\left\{a u_{i}, b u_{i}, d u_{i}\right\}$. For every $i \in\left[k^{\prime}\right]$, let $H_{i}=E\left(G\left[V_{q_{i}} \cup V_{q_{i}^{\prime}}\right]\right) \backslash E(a b d)$. Consider the following set.

$$
X^{\prime \prime}=X^{\prime} \backslash\left(\left\{F_{i}: i \in\left[m^{\prime}\right]\right\} \cup\left\{H_{i}: i \in\left[k^{\prime}\right]\right\}\right) \cup E(a b d) \cup\left\{v_{i} v_{i}^{\prime}: i \in\left[k^{\prime}\right]\right\} .
$$

Note that $X^{\prime \prime}$ is a transversal of $G^{\prime}$. If $E(\Delta) \subseteq X^{\prime}$, then we are done. So, we may assume that, for every $i \in\left[m^{\prime}\right],\left|X^{\prime} \cap F_{i}\right| \geq 1$; and, for every $i \in\left[k^{\prime}\right],\left|X^{\prime} \cap H_{i}\right| \geq 2$. Thus,

$$
\left|X^{\prime \prime}\right| \leq\left|X^{\prime}\right|-m^{\prime}-2 k^{\prime}+\left|E(\Delta) \backslash X^{\prime}\right|+k^{\prime} \leq\left|X^{\prime}\right|-m^{\prime}-k^{\prime}+2 \leq\left|X^{\prime}\right|,
$$


where the second inequality holds because $\Delta \cap X^{\prime} \neq \varnothing$, as $\Delta$ is a triangle in $G^{\prime}$ and $X^{\prime}$ is a transversal of $G^{\prime}$, and the last inequality holds because $m^{\prime}+k^{\prime}+1=\left|S^{\Delta}(t)\right| \geq 3$. Hence $X^{\prime \prime}$ is a minimum transversal of $G^{\prime}$ that contains $a b, b d$, and $a d$, and we are done.

Claim 5.17. Let $\Delta$ be a triple of vertices in $V_{t}$ with $\Delta \neq V_{t} \cap V_{p(t)}$. Then $\left|S^{\Delta}(t)\right| \leq 2$.

Proof. Without loss of generality, let $\Delta=a b d$. Suppose by contradiction that $\left|S^{\Delta}(t)\right| \geq 3$. First suppose that there exists a node in $S^{\Delta}(t) \cap L$. Without loss of generality, let $\ell_{1}$ be such node and let $G^{\prime}=G-u_{1}$. By Claim 5.16, there exists a minimum transversal $X^{\prime}$ of $G^{\prime}$ such that $\left|X^{\prime} \cap E(\Delta)\right|=3$. As $G$ is minimal, there exists a packing $Y^{\prime}$ in $G^{\prime}$ such that $\left(X^{\prime}, Y^{\prime}\right)$ is a $\frac{9}{5}$-TP of $G^{\prime}$. But then $\left(X^{\prime}, Y^{\prime}\right)$ is also a $\frac{9}{5}$-TP of $G$, a contradiction. Now suppose that there exists a node in $S^{\Delta}(t) \cap Q$. Without loss of generality, let $q_{1}$ be such node and let $G^{\prime}=G-v_{1}-v_{1}^{\prime}$. By Claim 5.16, there exists a minimum transversal $X^{\prime}$ of $G^{\prime}$ such that $\left|X^{\prime} \cap E(\Delta)\right|=3$. As $G$ is minimal, there exists a packing $Y^{\prime}$ in $G^{\prime}$ such that $\left(X^{\prime}, Y^{\prime}\right)$ is a $\frac{9}{5}$-TP of $G^{\prime}$. But then $\left(X^{\prime} \cup\left\{v_{1} v_{1}^{\prime}\right\}, Y^{\prime} \cup\left\{v_{1} v_{1}^{\prime} w_{1}\right\}\right)$ is also a $\frac{9}{5}$-TP of $G$, where $w_{1}$ is a vertex adjacent to both $v_{1}$ and $v_{1}^{\prime}$, a contradiction.

Claim 5.18. Let $\Delta$ be a triple of vertices in $V_{t}$ with $\Delta \neq V_{t} \cap V_{p(t)}$. If $S^{\Delta}(t)=\left\{t_{1}, t_{2}\right\}$, then either $S^{\Delta}(t) \cap L=\varnothing$ or $S^{\Delta}(t) \cap Q=\varnothing$.

Proof. Suppose by contradiction, and without loss of generality, that $\Delta=a b d$ and $\ell_{1}, q_{1} \in$ $S^{\Delta}(t)$. Without loss of generality, suppose that $V_{q_{1}} \cap V_{q_{1}^{\prime}}=\left\{b, d, v_{1}\right\}$. Let $G^{\prime}=G-v_{1}^{\prime}$. As $G$ is minimal, there exists a $\frac{9}{5}-\mathrm{TP}$, say $\left(X^{\prime}, Y^{\prime}\right)$, of $G^{\prime}$. Without loss of generality, assume that $b d \in X^{\prime} \cap E\left(b d v_{1}\right)$. Suppose for a moment that there exists an edge, say $b d$, of $b d v_{1}$ that is not in $Y^{\prime}$. Then $\left(X^{\prime} \cup\left\{v_{1} v_{1}^{\prime}\right\}, Y^{\prime} \cup\left\{b d v_{1}^{\prime}\right\}\right)$ is a $\frac{9}{5}$-TP of $G$, a contradiction. Thus, every edge of $b d v_{1}$ is in $Y^{\prime}$. As $d_{G^{\prime}}\left(v_{1}\right)=3$, we must have that $b d v_{1} \in Y^{\prime}$ and that $a v_{1} \notin E\left(Y^{\prime}\right)$. Suppose for a moment that both of $\left\{b u_{1}, d u_{1}\right\}$ are not in $Y^{\prime}$. Then $\left(X^{\prime} \cup\left\{v_{1} v_{1}^{\prime}\right\}, Y^{\prime} \backslash\left\{b d v_{1}\right\} \cup\left\{b v_{1} v_{1}^{\prime}, b d u_{1}\right\}\right)$ is a $\frac{9}{5}$-TP of $G$, a contradiction. Hence, at least one of $\left\{b u_{1}, d u_{1}\right\}$, say $b u_{1}$, is in $Y^{\prime}$. This implies, as $d_{G^{\prime}}\left(u_{1}\right)=3$, that $a b u_{1} \in Y^{\prime}$ and that $d u_{1} \notin E\left(Y^{\prime}\right)$. But then, $\left(X^{\prime} \cup\left\{v_{1} v_{1}^{\prime}\right\}, Y^{\prime} \backslash\left\{b d v_{1}, a b u_{1}\right\} \cup\left\{d v_{1} v_{1}^{\prime}, a b v_{1}, b d u_{1}\right\}\right)$ is a $\frac{9}{5}$-TP of $G$, a contradiction.

We now continue with the main proof of the theorem. For every triple of vertices $\Delta \subseteq V_{t}$ such that $\Delta \neq V_{t} \cap V_{p(t)}$, we abuse notation and let $h(\Delta)=1+\max \left\{h\left(t^{\prime}\right): t^{\prime} \in S^{\Delta}(t)\right\}$. We divide the rest of the proof in three cases, depending on how many triples of vertices $\Delta \subseteq V_{t}$ have $h(\Delta)=2$.

Case 1: $\left|\left\{\Delta \subseteq V_{t}: h(\Delta)=2\right\}\right|=1$. 
Without loss of generality, we may assume that $h(a b c)=2$ and that $V_{q_{1}} \cap V_{t}=\{a, b, c\}$. We define

$$
Y_{1}= \begin{cases}\left\{a c v_{1}, b v_{1} v_{1}^{\prime}\right\} & \text { if } V_{q_{1}^{\prime}} \cap V_{q_{1}}=\left\{a, b, v_{1}\right\}, \\ \left\{a b v_{1}, c v_{1} v_{1}^{\prime}\right\} & \text { if } V_{q_{1}^{\prime}} \cap V_{q_{1}}=\left\{a, c, v_{1}\right\} \text { or } V_{q_{1}^{\prime}} \cap V_{q_{1}}=\left\{b, c, v_{1}\right\} .\end{cases}
$$

By Claim 5.17 and Claim 5.18, we may assume, without loss of generality, that either $S^{a b c}(t)=\left\{q_{1}\right\}$ or $S^{a b c}(t)=\left\{q_{1}, q_{2}\right\}$. In the former case, we let $Y_{2}=Y_{1}$ and, in the later case, we let $Y_{2}=Y_{1} \cup\left\{v_{2} v_{2}^{\prime} w_{2}\right\}$, where $w_{2}$ is a vertex adjacent to both $v_{2}$ and $v_{2}^{\prime}$. Note that $\left|Y_{2}\right|=1+k$.

Let $G^{\prime}=G-R(t)$. By Claim 5.17, we have $\left|S^{a b d}(t)\right|,\left|S^{b c d}(t)\right| \leq 2$. By Claim 5.18. we have that $\left|S^{a b c}(t) \cap L\right|=0$. Thus we have $0 \leq m \leq 4$. In any case, we show that there exists a packing $Y_{3}$ of cardinality $1+k+\lceil m / 2\rceil$ in $G-E\left(G^{\prime}\right)$. If $m=0$ then we let $Y_{3}=Y_{2}$. If either $m=1$ or $m=2$ then we let, without loss of generality, $Y_{3}=$ $Y_{2} \cup\left\{a d u_{1}\right\}$. If either $m=3$ or $m=4$ then, without loss of generality we may assume that $V_{\ell_{1}} \cap V_{t}=\{a, b, d\}$ and that $V_{\ell_{2}} \cap V_{t}=\{a, c, d\}$. In that case, if $V_{q_{1}^{\prime}} \cap V_{q_{1}}=\left\{a, b, v_{1}\right\}$ then we let $Y_{3}=Y_{2} \cup\left\{a b u_{1}, a d u_{2}\right\}$ (Figure 5.7(a)) and, if either $V_{q_{1}}=\left\{a, c, v_{1}\right\}$ or $V_{q_{1}^{\prime}} \cap$ $V_{q_{1}}=\left\{b, c, v_{1}\right\}$, then we let $Y_{3}=Y_{2} \cup\left\{a d u_{1}, a c u_{2}\right\}$ (Figure 5.7(b) and Figure 5.7(c)).

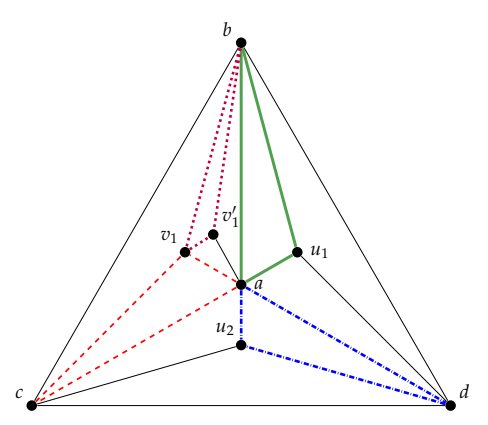

(a)

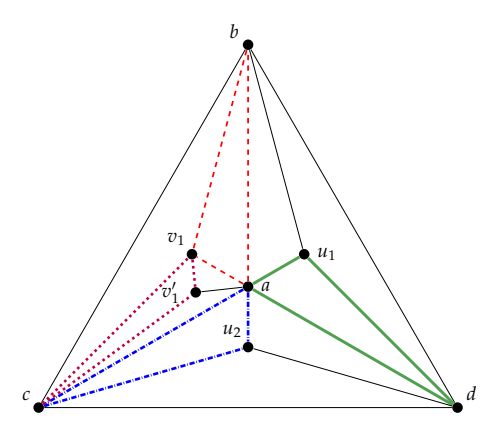

(b)

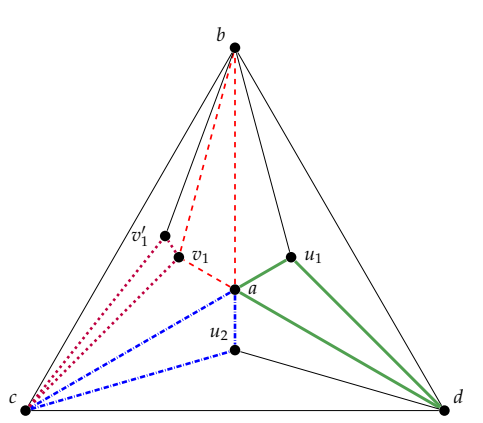

(c)

Figure 5.7: Situations in Case 1 of Theorem 5.10

Let $\left(X^{\prime}, Y^{\prime}\right)$ be a $\frac{9}{5}$-TP of $G^{\prime}$. By Claim 5.14 there exists a transversal $X$ of $G$ with cardinality $\left|X^{\prime}\right|+2 k+m$. Also, $Y=Y^{\prime} \cup Y_{3}$ is a packing in $G$ of cardinality $\left|Y^{\prime}\right|+1+$ $k+\lceil m / 2\rceil$.

Note that, as $m \leq 4$ we have that $m \leq 2 / 5+9 m / 10 \leq 2 / 5+9\lceil m / 2\rceil / 5$. Also, as $k \leq 2$, we have that $1+2 k \leq 9 k / 5+7 / 5$. So, $1+2 k+m \leq 9 / 5(1+k+\lceil m / 2\rceil)$. Thus, $(X, Y)$ is a $\frac{9}{5}$-TP in $G$, a contradiction.

Case 2: $\left|\left\{\Delta \subseteq V_{t}: h(\Delta)=2\right\}\right|=2$.

Without loss of generality, we may assume that $V_{q_{1}} \cap V_{t}=\{a, b, c\}$ and 
that $V_{q_{2}} \cap V_{t}=\{a, b, d\}$. We define $Y_{1}$ as in Case 1 , that is

$$
Y_{1}= \begin{cases}\left\{a c v_{1}, b v_{1} v_{1}^{\prime}\right\} & \text { if } V_{q_{1}^{\prime}} \cap V_{q_{1}}=\left\{a, b, v_{1}\right\}, \\ \left\{a b v_{1}, c v_{1} v_{1}^{\prime}\right\} & \text { if } V_{q_{1}^{\prime}} \cap V_{q_{1}}=\left\{a, c, v_{1}\right\} \text { or } V_{q_{1}^{\prime}} \cap V_{q_{1}}=\left\{b, c, v_{1}\right\} .\end{cases}
$$

If $a b \notin E\left(Y_{1}\right)$, then we let

$$
Y_{2}=Y_{1} \cup \begin{cases}\left\{a b v_{2}^{\prime}, a d v_{2}\right\} & \text { if } V_{q_{2}^{\prime}} \cap V_{q_{2}}=\left\{a, b, v_{2}\right\} \\ \left\{a b v_{2}, v_{2} v_{2}^{\prime} d\right\} & \text { if } V_{q_{2}^{\prime}} \cap V_{q_{2}}=\left\{a, d, v_{2}\right\} \text { or } V_{q_{2}^{\prime}} \cap V_{q_{2}}=\left\{b, d, v_{2}\right\}\end{cases}
$$

(Figure 5.8). If $a b \in E\left(Y_{1}\right)$, then we let $Y_{2}=Y_{1} \cup\left\{w_{2} v_{2} v_{2}^{\prime}\right\}$, where $w_{2}$ is a vertex adjacent to both $v_{2}$ and $v_{2}^{\prime}$ (Figure 5.9).

Let $G^{\prime}=G-R(t)$. Observe that $Y_{2}$ is a packing in $G-E\left(G^{\prime}\right)$. Moreover, $\left|Y_{2}\right| \in\{3,4\}$, and if $\left|Y_{2}\right|=3$, then $a c$, ad $\notin E\left(Y_{2}\right)$.

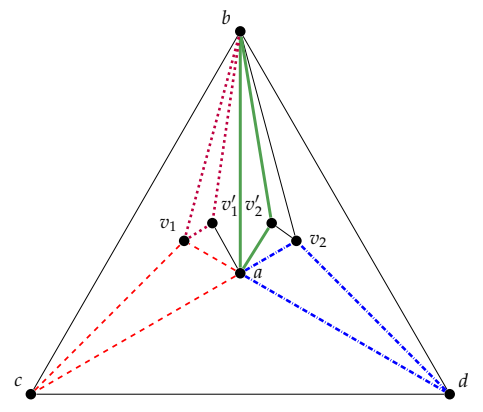

(a)

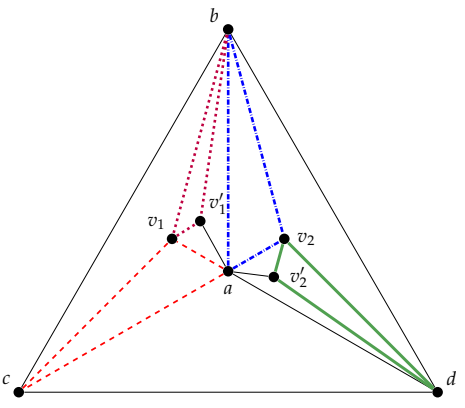

(b)

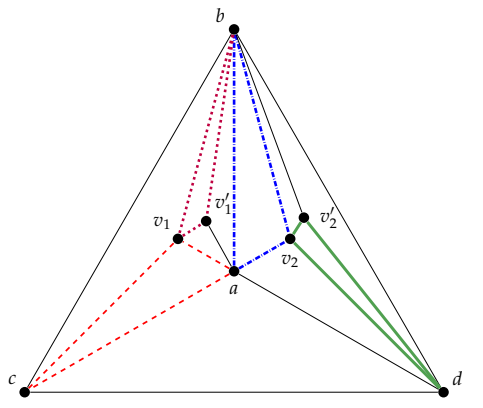

(c)

Figure 5.8: Situations in Case 2 of Theorem 5.10 when $a b \notin E\left(Y_{1}\right)$.

By Claims 5.17 and $5.18, m \leq 2$. We define

$$
Y_{3}= \begin{cases}Y_{2} & \text { if } m=0 \\ Y_{2} & \text { if } m=1 \text { and }\left|Y_{2}\right|=4 \\ Y_{2} \cup\left\{a c u_{1}\right\} & \text { if } m=1 \text { and }\left|Y_{2}\right|=3 \\ Y_{2} & \text { if } m=2 \text { and }\left|Y_{2}\right|=4 \\ Y_{2} \cup\left\{a c u_{1}, a d u_{2}\right\} & \text { if } m=2 \text { and }\left|Y_{2}\right|=3 .\end{cases}
$$

Observe that $\left|Y_{3}\right| \geq 3+\min \{1, m\}$.

For every $q_{i} \in S^{a b c}(t) \cup S^{a b d}(t) \backslash\left\{q_{1}, q_{2}\right\}$, let $w_{i}$ be an arbitrary vertex in $\{a, b, c, d\}$ adjacent to both $v_{i}$ and $v_{i}^{\prime}$. We let $Y_{4}=Y_{3} \cup\left\{v_{i} v_{i}^{\prime} w_{i}: q_{i} \in S^{a b c}(t) \cup S^{a b d}(t) \backslash\left\{q_{1}, q_{2}\right\}\right\}$. Note that $\left|Y_{4}\right| \geq 1+k+\min \{1, m\}$.

As $G$ is minimal, there exists a $\frac{9}{5}$-TP of $G^{\prime}$, say $\left(X^{\prime}, Y^{\prime}\right)$. So, $Y=Y^{\prime} \cup Y_{4}$ is a packing in $G$ of cardinality at least $\left|Y^{\prime}\right|+1+k+\min \{1, m\}$. By Claims 5.14 and 5.15 , there exists a transversal $X$ of $G$ with cardinality $\left|X^{\prime}\right|+\min \{1+m+2 k, 5+k\}$. By Claims 5.17 


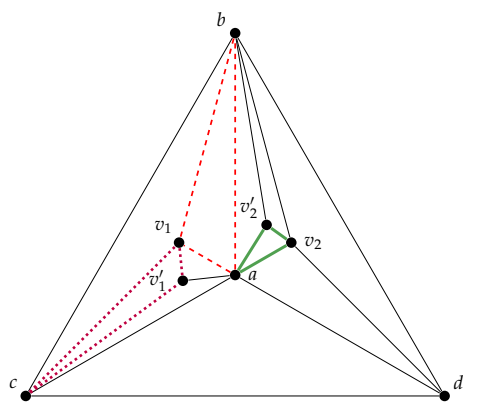

(a)

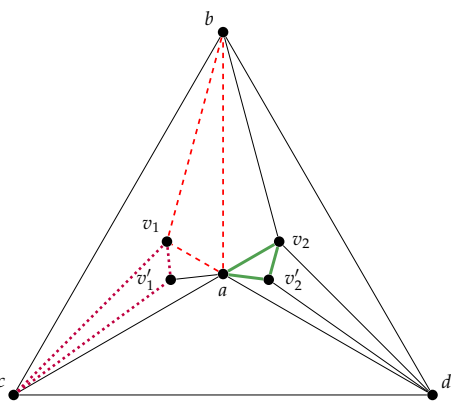

(b)

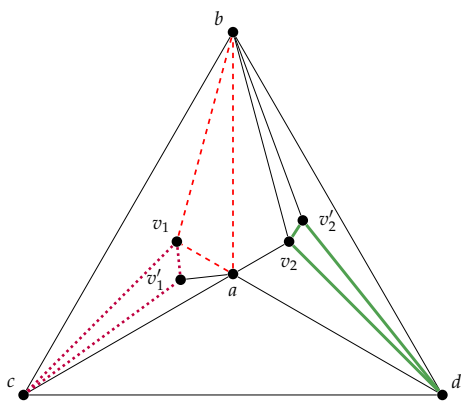

(c)

Figure 5.9: Situations in Case 2 of Theorem 5.10 when $a b \in E\left(Y_{1}\right)$.

and 5.18, $2 \leq k \leq 4$ and $m \leq 2$, so $\min \{1+m+2 k, 5+k\} \leq \frac{9}{5}(1+k+\min \{1, m\})$. Thus, $(X, Y)$ is a $\frac{9}{5}$-TP in $G$, a contradiction.

Case 3: $\left|\left\{\Delta \subseteq V_{t}: h(\Delta)=2\right\}\right|=3$.

Without loss of generality, we may assume that $V_{q_{1}} \cap V_{t}=\{a, b, c\}$ and that $V_{q_{2}} \cap V_{t}=\{a, b, d\}$. We define $Y_{1}$ and $Y_{2}$ as in Case 2. Let $q_{3} \in S^{a c d}(t)$. We let

$$
Y_{3}=Y_{2} \cup \begin{cases}\left\{c v_{3} v_{3}^{\prime}\right\} & \text { if } V_{q_{3}^{\prime}} \cap V_{q_{3}}=\left\{a, c, v_{3}\right\} \text { or } V_{q_{3}^{\prime}} \cap V_{q_{3}}=\left\{c, d, v_{3}\right\} \\ \left\{d v_{3} v_{3}^{\prime}\right\} & \text { if } V_{q_{3}^{\prime}} \cap V_{q_{3}}=\left\{a, d, v_{3}\right\}\end{cases}
$$

and

$$
Y_{4}=Y_{3} \cup \begin{cases}\left\{a d v_{3}\right\} & \text { if }\left|Y_{2}\right|=3 \text { and } Y_{3} \backslash Y_{2}=\left\{c v_{3} v_{3}^{\prime}\right\} \\ \left\{a c v_{3}\right\} & \text { if }\left|Y_{2}\right|=3 \text { and } Y_{3} \backslash Y_{2}=\left\{d v_{3} v_{3}^{\prime}\right\} \\ \varnothing & \text { if }\left|Y_{2}\right|=4\end{cases}
$$

Note that $Y_{4}$ is a packing of cardinality 5 in $G-E\left(G^{\prime}\right)$ (Figure 5.10). For ev-

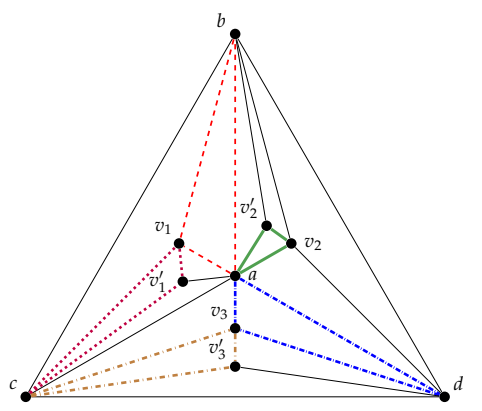

(a)

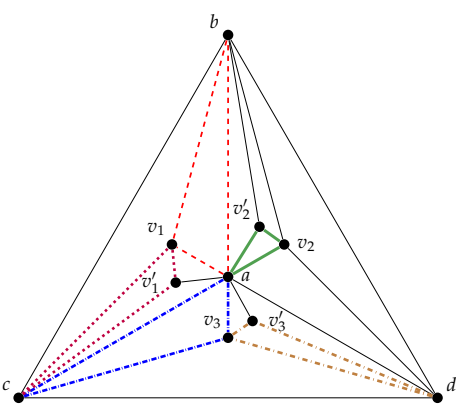

(b)

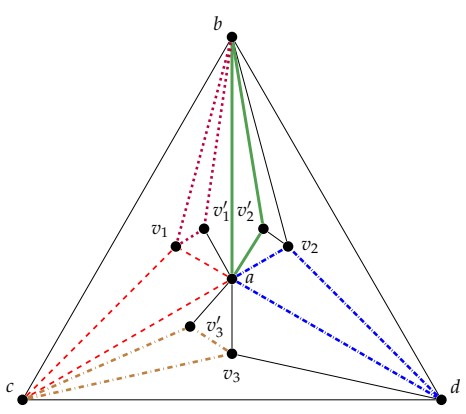

(c)

Figure 5.10: Situations in Case 3 of Theorem 5.10

ery $q_{i} \in S^{a b c} \cup S^{a b d} \cup S^{a c d} \backslash\left\{q_{1}, q_{2}, q_{3}\right\}$, let $w_{i}$ be an arbitrary vertex adjacent to both $v_{i}$ and $v_{i}^{\prime}$. We let $Y_{5}=Y_{4} \cup\left\{v_{i} v_{i}^{\prime} w_{i}: q_{i} \in S^{a b c} \cup S^{a b d} \cup S^{a c d} \backslash\left\{q_{1}, q_{2}, q_{3}\right\}\right\}$. Note that $\left|Y_{5}\right| \geq$ $2+k$. As $G$ is minimal, there exists a $\frac{9}{5}-\mathrm{TP}$, say $\left(X^{\prime}, Y^{\prime}\right)$, of $G^{\prime}$. By Claim 5.14 , there exists 
a transversal $X$ of $G$ with cardinality $\left|X^{\prime}\right|+1+2 k$ in $G$. Also, $Y=Y^{\prime} \cup Y_{5}$ is a packing in $G$ of cardinality at least $\left|Y^{\prime}\right|+2+k$. Thus, because $\frac{1+2 k}{2+k} \leq \frac{9}{5},(X, Y)$ is a $\frac{9}{5}$-TP in $G$, a contradiction.

This concludes the proof of the theorem.

A natural question asks whether the bound stated in Theorem 5.10 can be improved when the 3-tree is also planar. The next section show that this can be done to a broader class of planar graphs.

\subsection{Planar Triangulations}

In this section, we show that $\tau(G) \leq \frac{3}{2} \nu(G)$ for every planar triangulation $G$ different from $K_{4}$ (Theorem 5.25). We begin by introducing new definitions. A graph is planar if it can be drawn in the plane so that its edges intersect only at their ends. Such a drawing is called a planar embedding of the graph, and the regions obtained by dividing the plane by such a drawing are called the faces of the planar embedding.

We say that a connected graph is a planar triangulation if it has a planar embedding in which all of its faces are triangles. Fixed a planar embedding of a planar triangulation $G$ with at least four vertices, the dual graph $G^{*}$ of $G$ is the graph whose vertex set is the set of faces of the embedding, and in which two vertices are adjacent if and only if their corresponding faces intersect ${ }^{1}$

A cubic graph is a graph in which all its vertices have degree 3. The following proposition comes naturally. See [BM08, Proposition 10.11] and [BM08, Exercise 10.2.6].

Proposition 5.19. The dual of planar triangulation with at least four vertices is cubic and has no bridges.

Our proof is divided in two parts. In Lemma 5.22, we give an upper bound for $\tau(G)$ and in Lemma 5.24 we give a corresponding lower bound for $v(G)$. For the first part, we need the following theorem of Petersen and Euler's formula.

Proposition 5.20 ([Pet91]). Every bridgeless cubic graph contains a perfect matching.

Proposition 5.21 ([BM08]). Let $f$ be the number of faces in a connected planar graph $G$, then $f=|E(G)|-|V(G)|+2$.

Lemma 5.22. If $G$ is a planar graph with $V(G)>1$, then $\tau(G) \leq n-2$.

\footnotetext{
${ }^{1}$ There is a broader definition for the dual of planar graphs, but it implies working with multigraphs, that is, graphs that admit self-loops and parallel edges. This broader definition implies to add an edge in the dual for every edge that the corresponding faces share. In this text we do not use it, as we are only interested in planar triangulations, and it is known that the dual (in this broader sense) of any planar triangulation with at least four vertices, is always a simple graph [BM08, Exercise 10.2.6]
} 
Proof. Let $G^{\prime}$ be a maximal planar graph containing $G$ with $V(G)=V\left(G^{\prime}\right)$. If $V(G)<4$, then the proof is straightforward. So, we may assume that $V(G) \geq 4$. Note that $\tau(G) \leq \tau\left(G^{\prime}\right)$. Let $f$ be the number of faces in $G^{\prime}$. As $G^{\prime}$ is maximal, $G^{\prime}$ is connected and all faces in $G^{\prime}$ are triangles. Thus, $\left|E\left(G^{\prime}\right)\right|=\frac{3}{2} f$ and, by Proposition 5.21, $f=2 n-4$. Since $G^{\prime}$ is a planar triangulation with at least four vertices, by Proposition 5.19, the dual $G^{*}$ of $G^{\prime}$ is a cubic graph on $2 n-4$ vertices. Moreover, $G^{*}$ is bridgeless by Proposition 5.19. Thus, by Proposition 5.20, $G^{*}$ contains a perfect matching $M^{*}$.

Let $M^{\prime}$ be the edges of $G^{\prime}$ corresponding to the edges in $M^{*}$. Note that $M^{\prime}$ is a transversal of $G^{\prime}$. Note also that $\left|M^{\prime}\right|=\left|M^{*}\right|=\left|V\left(G^{*}\right)\right| / 2=f / 2=n-2$. Hence $\tau\left(G^{\prime}\right) \leq\left|M^{\prime}\right|=n-2$.

Observe that Lemma 5.22 is valid for all planar graphs, not only for planar triangulations. We now proceed to prove a lower bound for $v(G)$. Before this, we state the well-known Brook's theorem.

Proposition 5.23 ([Bro41] $)$. If $G$ is a connected graph that is neither an odd cycle nor a complete graph, then $G$ is $\Delta$-partite, where $\Delta$ is the maximum degree of $G$.

Lemma 5.24. If $G$ is a planar triangulation different from $K_{4}$, then $v(G) \geq \frac{2}{3}(n-2)$.

Proof. Let $G^{*}$ be the dual graph of $G$. Since $G$ is a planar triangulation different from $K_{4}, G^{*}$ is also different from $K_{4}$. Thus, as $G^{*}$ is cubic by Proposition 5.19, $G^{*}$ is neither an odd cycle nor a complete graph. Thus, by Proposition 5.23, there exists an independent set $Y^{*}$ of $G^{*}$ with cardinality at least $\frac{\left|V\left(G^{*}\right)\right|}{3}$. Let $Y$ be the faces of $G$ corresponding to the vertices in $Y^{*}$. As $Y^{*}$ is an independent set in $G^{*}, Y$ is a packing of $G$, and $v(G) \geq|Y|=\left|Y^{*}\right| \geq \frac{\left|V\left(G^{*}\right)\right|}{3}=\frac{2}{3}(n-2)$.

Finally, we show our main result.

Theorem 5.25. If $G$ is a planar triangulation different from $K_{4}$, then $\tau(G) \leq \frac{3}{2} v(G)$. Moreover, this bound is tight.

Proof. The first part of the proof follows directly from Lemmas 5.22 and 5.24 applied to G. For the second part, consider the graph $K_{5}-e$ (Figure 5.11). In this graph, $\tau(G)=3$ and $v(G)=2$.

Planar 3-trees are also planar triangulations. Moreover, the graph of Figure 5.11] is also a planar 3-tree. Hence, we have the following corollary.

Corollary 5.26. For every planar 3-tree $G$ different from $K_{4}, \tau(G) \leq \frac{3}{2} v(G)$. Moreover, this bound is tight. 


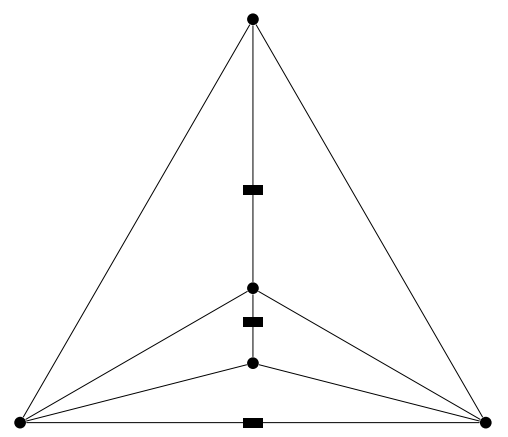

Figure 5.11: The graph $K_{5}-e$. This graph has a packing of cardinality 2 and a transversal of cardinality 3.

We finish this section by remarking that the graph of Figure 5.11 is not the only planar triangulation that has a bound of $3 / 2$. An interesting question is to obtain an infinite family of such graphs.

\subsection{Dense Tripartite Graphs}

Improving a previous bound of Tuza [Tuz90, Proposition 8], Haxell and Kohayakawa [HK98] showed that $\tau(G) \leq 1.956 v(G)$ for every tripartite graph $G$. This bound was improved by Szestopalow [Sze16, Theorem 4.1.5]. He showed that $\tau(G) \leq 1.87 \nu(G)$ if $G$ is tripartite.

Another result of Szestopalow [Sze16, Proposition 4.1.6] implies that, when $G$ is a complete tripartite graph, we have $\tau(G)=v(G)$ (see also [ALBT11, Proposition 11]). Tuza showed that Tuza's Conjecture holds if $G$ is a graph with $n$ vertices and at least $\frac{7 n^{2}}{16}$ edges [Tuz90, Theorem 5]. Motivated by this result, we show a similar result when $G$ is a dense tripartite graph. (Theorem 5.30 and Corollary 5.31).

Recall that an edge-coloring of a graph $G$ is a collection $\left\{M_{1}, M_{2}, \ldots, M_{k}\right\}$ of pairwise disjoint matchings in $G$ such that $M_{1} \cup M_{2} \cup \ldots \cup M_{k}=E(G)$ (Section 2.1). We start by stating two important propositions. The first one is the well-known Theorem of König.

Proposition 5.27 ([Kö16]). Every bipartite graph admits an edge-coloring with cardinality $\Delta$, where $\Delta$ is the maximum degree of the graph.

The next proposition appears implicitly in the proof of a result of Aparna et al. [ALBT11, Proposition 11].

Proposition 5.28. Every complete tripartite graph $G=(A, B, C, E(G))$ with $|A|=a \geq|B|=b \geq|C|=c$ has a packing of cardinality $b c$.

Proof. By Proposition 5.27, there is an edge-coloring of cardinality $b$ in the bipartite graph $G[B \cup C]$. Let $\left\{M_{1}, M_{2}, \ldots, M_{b}\right\}$ be such edge-coloring. As every matching in $G[B \cup C]$ has cardinality at most $c$, and the number of edges in $G[B \cup C]$ is $b c$, we 
have $\left|M_{i}\right|=c$ for every $i \in\{1,2, \ldots, b\}$. Then we can extend this edge-coloring to form a packing of $G$ in the following way. Let $A=\left\{v_{1}, v_{2}, \ldots, v_{a}\right\}$. For every $i \in\{1,2, \ldots, b\}$, let $Y_{i}=\left\{v_{i} u w: u w \in M_{i}\right\}$. Observe that every $Y_{i}$ is a packing in $G$, as every $M_{i}$ is a matching in $G[B \cup C]$. Observe also that $\left|Y_{i}\right|=\left|M_{i}\right|=c$. Also, as every pair of elements in $\left\{M_{1}, M_{2}, \ldots, M_{b}\right\}$ is disjoint, $Y_{1} \cup Y_{2} \cup \ldots \cup Y_{b}$ is a packing in $G$ of cardinality $b c$.

The next lemma is an adaptation of a result of Győri and Tuza [GT87, Proposition 4] for the case of tripartite graphs.

Lemma 5.29. Every tripartite graph $G=(A, B, C, E(G))$ with $|A|=a \geq|B|=b \geq|C|=c$ has a packing of cardinality $t / a$, where $t$ is the number of triangles in $G$.

Proof. Let $n=a+b+c$. Let $G^{*}$ be the complete tripartite graph on $(A, B, C)$. By Proposition 5.28, there exists a packing $S$ of cardinality $s=b c$ in $G^{*}$. Let $\mathcal{T}$ be the set of all triangles in $G$. We say that a permutation $\pi$ of the vertices of $G$ is a 3-permutation if, for every $X \in\{A, B, C\}$ and for every $x \in X, \pi(x) \in X$. In other words, $\pi$ consists of a permutation of each side of the tripartition. It is clear that there are exactly $a ! \cdot b ! \cdot c !$ such permutations.

For an arbitrary 3-permutation $\pi$ of $V(G)$, put $t_{\pi}=|\mathcal{T} \cap \pi(S)|$, where $\pi(S)$ is the image of $S$ after applying $\pi$. Let $T \in \mathcal{T}$ and $T^{\prime}$ be a triangle in $G^{*}$. Observe that there are $(a-1) ! \cdot(b-1) ! \cdot(c-1)$ ! permutations such that $\pi\left(T^{\prime}\right)=T$. Choosing a 3-permutation uniformly at random, the expected value of $t_{\pi}$ is $t s / a b c$. Consequently, there exists a 3-permutation $\pi$ for which $\pi(S)$ is a packing of cardinality $t s / a b c=t / a$.

The following theorem is the main result of this section.

Theorem 5.30. Every tripartite graph $G$ with $n$ vertices and $m \geq \frac{n^{2}}{4}$ edges is such that $\tau(G) \leq \frac{n^{2}}{3 \cdot\left(4 m-n^{2}\right)} \cdot v(G)$.

Proof. Let $t$ be the number of triangles in G. By Bollobás [Bol78, Corollary 6.1.9] (see also [Fis89, Figure 1]), $t \geq \frac{n}{9}\left(4 m-n^{2}\right)$. So, by Lemma 5.29.

$$
v(G) \geq t / a \geq \frac{\left(4 m-n^{2}\right) n}{9 a} .
$$

Now, as $\tau(G) \leq b c$, we have that

$$
\tau(G) / \nu(G) \leq \frac{b c}{\frac{\left(4 m-n^{2}\right) n}{9 a}}=\frac{9 a b c}{\left(4 m-n^{2}\right) n} \leq \frac{\frac{n^{3}}{3}}{\left(4 m-n^{2}\right) n}=\frac{1}{3} \cdot \frac{n^{2}}{\left(4 m-n^{2}\right)},
$$

and the proof follows. 
As stated in the introduction of this section, given a tripartite graph $G$, the best known upper bound for $\tau(G) / v(G)$ is 1.87 and was given by Szestopalow [Sze16, Theorem 4.1.5]. The following corollary shows that this bound is improved if $G$ is dense enough.

Corollary 5.31. For any $\alpha>0$, every tripartite graph $G$ with at least $\left(\frac{1+3 \alpha}{12 \alpha}\right) n^{2}$ edges is such that $\tau(G) \leq \alpha \nu(G)$. In particular, if $G$ has at least $0.295 n^{2}$ edges then $\tau(G) \leq 1.86 v(G)$.

\subsection{Complete 4-Partite Graphs}

Aparna et al. [ALBT11, Corollary 7] showed that Tuza's Conjecture holds for 4-partite graphs. In this section, we improve this bound for the subclass of complete 4-partite graphs (Theorem 5.35). We begin by stating the well-known theorem of Vizing.

Proposition 5.32. [Viz89] (see also [Die10, Theorem 5.3.2]) Every graph admits an edgecoloring with cardinality $\Delta+1$, where $\Delta$ is the maximum degree of the graph.

The next lemma will help us to find a subgraph of a complete bipartite graph that is regular in some sense. Given a vertex $v$ in a graph $G$, we say that $d_{G}(v) \sim \ell$ if $d_{G}(v) \in\{\lfloor\ell\rfloor,\lceil\ell\rceil\}$.

Lemma 5.33. Let $G=(X, Y, E)$ be a complete bipartite graph with $x=|X|, y=|Y|$ and $x \geq y$. Let $k$ be a positive integer with $k \leq x$. There exists a subgraph of $G$, with the same vertex set of $G$, such that $d_{Y}(v)=k$ for every $v \in Y$ and $d_{X}(v) \sim y k / x$ for every $v \in X$.

Proof. Let $r=y k \bmod x$. Let $X=\left\{v_{0}, v_{1}, \ldots, v_{x-1}\right\}$ and $Y=\left\{w_{0}, w_{1}, \ldots, w_{y-1}\right\}$. We construct a capacited directed graph $D=(N, A, c)$ such that $N=X \cup Y \cup\{s, t\}$, and $A=\left\{v_{i} w_{j}: v_{i} \in X, w_{j} \in Y\right\} \cup\left\{s v_{i}: v_{i} \in X\right\} \cup\left\{w_{j} t: w_{j} \in Y\right\}, c\left(v_{i} w_{j}\right)=1$ if $v_{i} w_{j} \in E(G), c\left(s v_{i}\right)=\lceil y k / x\rceil$ if $i<r, c\left(s v_{i}\right)=\lfloor y k / x\rfloor$ if $i \geq r$, and $c\left(w_{j} t\right)=k$ for every $w_{j} \in Y$. We prove that every st-cut has capacity at least $k y$. Let $S$ be a set of nodes of $D$ that separates $s$ from $t$. If $|X \cap S| \geq k$, then $c(S) \geq k|Y \backslash S|+k|S \cap Y|=k|Y|$ and we are done. So, suppose that $|X \cap S|<k$. Then,

$$
\begin{aligned}
c(S) & =\sum_{v_{i} \in X \backslash S} c\left(s v_{i}\right)+|X \cap S| \cdot|Y \backslash S|+k|Y \cap S| \\
& =\sum_{v_{i} \in X} c\left(s v_{i}\right)-\sum_{v_{i} \in X \cap S} c\left(s v_{i}\right)+|X \cap S| \cdot|Y \backslash S|+k|Y \cap S| \\
& =k y-\sum_{v_{i} \in X \cap S} c\left(s v_{i}\right)+|X \cap S| \cdot|Y \backslash S|+k|Y \cap S| \\
& >k y-\sum_{v_{i} \in X \cap S}\lceil y k / x\rceil+|X \cap S| \cdot|Y \backslash S|+|X \cap S||Y \cap S| \\
& =k y-|X \cap S|\lceil y k / x\rceil+|X \cap S| \cdot|Y| \\
& =k y+|X \cap S|(y-\lceil y k / x\rceil)
\end{aligned}
$$




$$
\geq k y \text {. }
$$

As there exists a cut of value $k y$, by the max-flow min-cut theorem [FF56], there exists a flow of value $k y$. By Dantzig and Fulkerson [DF55], this flow is integer. We can convert this flow into the desired subgraph.

The next proposition shows how to convert edge-colorings in tripartite graphs into packings in 4-partite graphs. It can be viewed as a generalization of Proposition 5.28.

Proposition 5.34. Let $G=(A, B, C, D, E(G))$ be a complete 4-partite graph. If there exists a subgraph $G^{\prime}$ of $G[B \cup C \cup D]$ with $d_{G^{\prime}}(v) \leq|A|-1$ for all $v \in V\left(G^{\prime}\right)$, then $v(G) \geq\left|E\left(G^{\prime}\right)\right|$.

Proof. By Proposition 5.32, there exists an edge-coloring of $G^{\prime}$ with cardinality $a:=|A|$. Let $\left\{M_{1}, M_{2}, \ldots, M_{a}\right\}$ be such a coloring. We can extend each of these matchings to form a packing of $G$ in the following way. Let $A=\left\{v_{1}, v_{2}, \ldots, v_{a}\right\}$. For every $i \in$ $\{1,2, \ldots, a\}$, let $Y_{i}=\left\{v_{i} u w: u w \in M_{i}\right\}$. Observe that every $Y_{i}$ is a packing in $G$, as every $M_{i}$ is a matching in $G^{\prime}$. Observe also that $\left|Y_{i}\right|=\left|M_{i}\right|$. Also, as every pair of matchings in $\left\{M_{1}, M_{2}, \ldots, M_{a}\right\}$ is disjoint, and each matching forms triangles with a different vertex from $A, Y=Y_{1} \cup Y_{2} \cup \cdots \cup Y_{a}$ is a packing in $G$ of cardinality $\left|M_{1}\right|+\left|M_{2}\right|+\cdots+\left|M_{a}\right|=\left|E\left(G^{\prime}\right)\right|$.

Finally, we prove our main theorem.

Theorem 5.35. Let $G=(A, B, C, D, E(G))$ be a complete 4-partite graph with $a:=|A| \geq$ $b:=|B| \geq c:=|C| \geq d:=|D|$, and $a>1$.

- If $a \geq b+c+1$, then $\tau(G)=v(G)$.

- If $c+d<a \leq b+c$, then $\tau(G) \leq \frac{a d+b c}{a d+b c-d} \cdot v(G)$.

- If $a \leq c+d$, then $\tau(G) \leq \frac{2 a d+2 b c}{2 a d+a c-2 c-2 d} \cdot v(G)$.

Proof. For every $P, Q \in\{A, B, C, D\}$, we say that the $P Q$ edges are the edges of $G$ with one end in $P$ and the other in $Q$. We divide the proof in the three obvious cases.

Case 1: $a \geq b+c+1$.

By Proposition 5.34, with $G^{\prime}=G[B \cup C \cup D], v(G) \geq b c+c d+b d$. Also, the set of $B C$ edges joined to the set of $C D$ and $B D$ edges form a transversal of $G$, with cardinality $b c+c d+b d$. Thus $\tau(G) \leq b c+c d+b d \leq v(G)$.

Case 2: $c+d<a \leq b+c$.

By Lemma 5.33, with $k=a-b-1 \leq c$, there exists a subgraph $G_{C D}^{\prime}$ of $G[C \cup D]$ such that $d_{G_{C D}^{\prime}}(v)=a-b-1$ for every $v \in D$ and $d_{G_{C D}^{\prime}}(v) \sim \frac{d}{c}(a-b-1) \leq a-b-1$ for 
every $v \in C$. Let $G^{\prime}=G[B \cup D] \cup G[B \cup C] \cup G_{C D}^{\prime}$. Observe that, for every $v \in V\left(G^{\prime}\right)$, we have $d_{G^{\prime}}(v) \leq a-1$. Indeed, if $v \in B$, then $d_{G^{\prime}}(v)=c+d \leq a-1$; if $v \in C$, then $d_{G^{\prime}}(v) \leq b+(a-b-1)=a-1$; if $v \in D$, then $d_{G^{\prime}}(v)=b+(a-b-1)=a-1$. By Proposition 5.34, $v(G) \geq\left|E\left(G^{\prime}\right)\right|=b c+b d+(a-b-1) d=b c+a d-d$. Also, the set of $B C$ edges joined to the set of $A D$ edges form a transversal of $G$, with cardinality $a d+b c$. Hence, $\tau(G) \leq a d+b c \leq \frac{a d+b c}{a d+b c-d} v(G)$.

Case 3: $a \leq c+d$.

By Lemma 5.33, with $k=\left\lfloor\frac{a-1}{2}\right\rfloor \leq c$, there exists a subgraph $G_{C D}^{\prime}$ of $G[C \cup D]$ such that $d_{G_{C D}^{\prime}}(v)=\left\lfloor\frac{a-1}{2}\right\rfloor$ for every $v \in D$, and $d_{G_{C D}^{\prime}}(v) \sim \frac{d}{c}\left(\left\lfloor\frac{a-1}{2}\right\rfloor\right) \leq\left\lfloor\frac{a-1}{2}\right\rfloor$ for every $v \in C$. By Lemma 5.33, with $k=\left\lceil\frac{a-1}{2}\right\rceil \leq b$, there exists a subgraph $G_{B D}^{\prime}$ of $G[B \cup D]$ such that $d_{G_{B D}^{\prime}}(v)=\left\lceil\frac{a-1}{2}\right\rceil$ for every $v \in D$, and $d_{G_{C D}^{\prime}}(v) \sim \frac{d}{b}\left(\left\lceil\frac{a-1}{2}\right\rceil\right) \leq\left\lceil\frac{a-1}{2}\right\rceil$ for every $v \in B$. By Lemma 5.33, with $k=\left\lfloor\frac{a-1}{2}\right\rfloor \leq b$, there exists a subgraph $G_{B C}^{\prime}$ of $G[B \cup C]$ such that $d_{G_{B C}^{\prime}}(v)=\left\lfloor\frac{a-1}{2}\right\rfloor$ for every $v \in C$, and $d_{G_{B C}^{\prime}}(v) \sim \frac{b}{c}\left(\left\lfloor\frac{a-1}{2}\right\rfloor\right) \leq\left\lfloor\frac{a-1}{2}\right\rfloor$ for every $v \in B$. Let $G^{\prime}=G_{B D}^{\prime} \cup G_{B C}^{\prime} \cup G_{C D}^{\prime}$.

Observe that, for every $v \in V\left(G^{\prime}\right)$, we have $d_{G^{\prime}}(v) \leq a-1$. Indeed, if $v \in B$, then $d_{G^{\prime}}(v)=d_{G_{B C}^{\prime}}(v)+d_{G_{B D}^{\prime}}(v) \leq\left\lfloor\frac{a-1}{2}\right\rfloor+\left\lceil\frac{a-1}{2}\right\rceil=a-1$; if $v \in C$, then $d_{G^{\prime}}(v)=$ $d_{G_{B C}^{\prime}}(v)+d_{G_{C D}^{\prime}}(v) \leq 2\left\lfloor\frac{a-1}{2}\right\rfloor \leq a-1$; if $v \in D$, then $d_{G^{\prime}}(v)=d_{G_{B D}^{\prime}}(v)+d_{G_{C D}^{\prime}}(v)=$ $\left\lfloor\frac{a-1}{2}\right\rfloor+\left\lceil\frac{a-1}{2}\right\rceil=a-1$. Thus, by Proposition 5.34 .

$$
\begin{aligned}
v(G) & \geq\left|E\left(G^{\prime}\right)\right| \\
& =\left|E\left(G_{B C}^{\prime}\right)\right|+\left|E\left(G_{C D}^{\prime}\right)\right|+\left|E\left(G_{B D}^{\prime}\right)\right| \\
& =c\left\lfloor\frac{a-1}{2}\right\rfloor+d\left\lfloor\frac{a-1}{2}\right\rfloor+d\left\lceil\frac{a-1}{2}\right\rceil \\
& =c\left\lfloor\frac{a-1}{2}\right\rfloor+d(a-1) \\
& \geq c\left(\frac{a}{2}-1\right)+d(a-1) \\
& =a d+\frac{a c}{2}-c-d .
\end{aligned}
$$

Also, the set of $B C$ edges joined to the set of $A D$ edges form a transversal of $G$, with cardinality $a d+b c$. Hence, as $a>1, \tau(G) \leq a d+b c \leq \frac{2 a d+2 b c}{2 a d+a c-2 c-2 d} \cdot v(G)$.

This concludes the proof of the theorem.

\subsection{Remarks}

In this chapter we showed upper bounds for the quotient $\tau(G) / \nu(G)$ for different classes of graphs. When the corresponding graph has treewidth at most 6 (Section 5.2), the stated bound is exactly the bound established in Tuza's Conjecture. In the rest of the classes, the corresponding bound is better than 2. Hence, they can be seen as bounds to stronger versions of Tuza's Conjecture. 
Several interesting questions are open. It remains to verify Tuza's Conjecture for partial $k$-trees with $k \geq 7$. We believe that the present technique can be used to find a result when $k=7$. For $k \geq 8$, more advanced techniques must be discovered. Also, we believe that the technique shown for 3-trees can be extended to show new results for the class of $k$-trees. Moreover, we have not shown that the bound of $\frac{9}{5}$ for 3 -trees is tight. In fact, we conjecture that a bound of $\frac{3}{2}$ holds for 3-trees. The graph $K_{5}-e$ is not the only graph that makes Theorem 5.25 tight. Two interest questions are to find an infinite family of such graphs and characterize the planar triangulations for which the corresponding bound is $\frac{3}{2}$.

Theorem 5.10 and Corollary 5.8 give us a direction to work on the class of chordal graphs. At the moment, no result for the entire class of chordal graphs has been proved. It seems difficult to prove Tuza's Conjecture even for split graphs. Finally, we believe our techniques for dense 3-partite graphs and complete 4-partite graphs can be extended to find a result for the dense $k$-partite graphs and for general dense graphs. 


\section{Chapter 6}

\section{Conclusions and Future Work}

In this thesis, we study three types of transversals: transversals of longest paths, transversals of longest cycles, and transversals of triangles. Our results imply an advance to the present literature on the subject. However, there remains many open questions to solve. In this final chapter, we review the results on the literature and propose lines of research for future open problems.

\subsection{Longest Paths and Longest Cycles}

New results for several classes of graphs can be obtained. In Figure 6.1 and Table 6.1 we show maps for the classes that have been studied for longest paths and longest cycles. They show us good insights on what steps this research can be continued. We present them as open problems. Some of them are present as conjectures, as we believe they are true. New techniques, or extensions of the ones presented in this work, should be developed to solve these open questions.

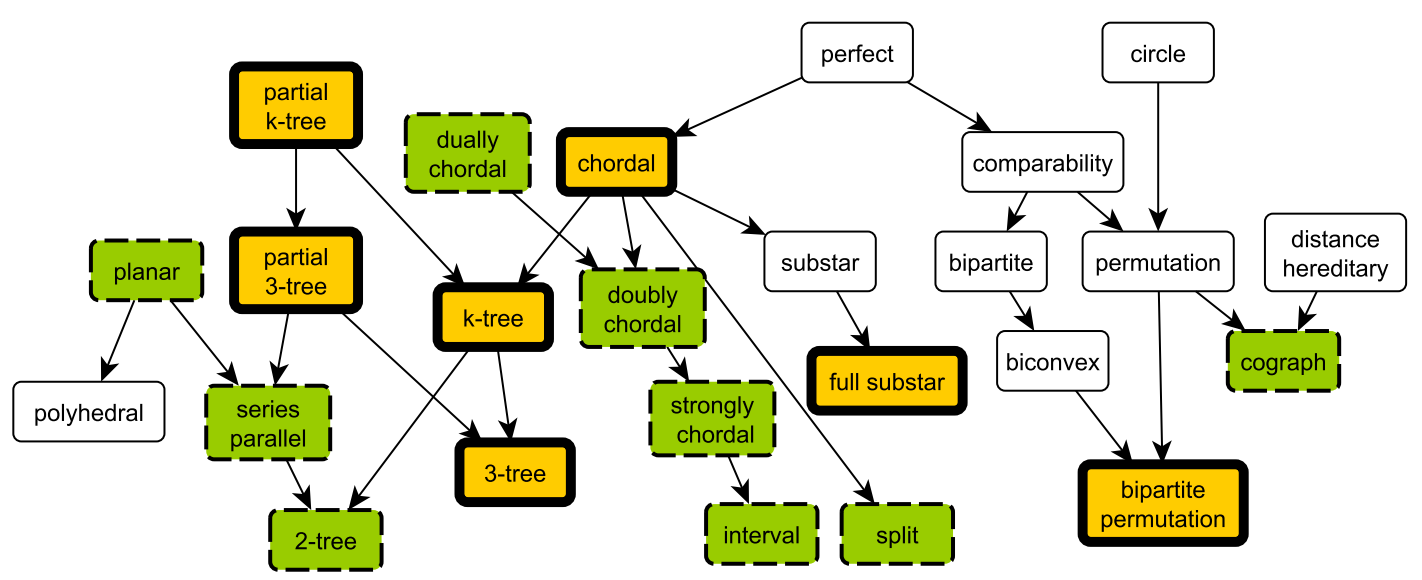

Figure 6.1: A map of the graph classes for lpt and lct. An arrow from a class $A$ to a class $B$ indicates that $B$ is a subclass of $A$. There are results for the classes with a dashed line and they are due to other people. We have results for the classes with a thick line. The classes with thin straight line have not yet been studied. 


\begin{tabular}{|c|c|c|c|c|}
\hline Class & $1 p t$ & References & lct & References \\
\hline Arbitrary & {$[n / 47$} & [RS14] & {$[n / 3\rceil$} & [RS14] \\
\hline Partial $k$-tree & $k$ & Section 3.2, $\left[\mathrm{CFG}^{+} 17\right]$ & $k-1$ & Section 4.3, [Gut18] \\
\hline Partial 3-tree & $\{2,3\}$ & Corollary 3.26 & 1 & Section 4.4, [Gut18] \\
\hline Partial 4-tree & $\{2,3,4\}$ & Theorem 3.10 & $\{2,3\}$ & Corollary 4.37 \\
\hline Chordal & $\omega-2$ & Section 3.1, $\left[\mathrm{CFG}^{+} 17\right]$ & $\omega-3$ & Section 4.2, [Gut18] \\
\hline$k$-tree & $k-1$ & Corollary 3.6 & $k-2$ & Corollary 4.14 \\
\hline 3-tree & $\{1,2\}$ & Corollary $\overline{\overline{3.6}}$ & 1 & Corollary $\overline{4.15}$ \\
\hline Bip. Permutation & 1 & Section 3.4, $\left[\overline{\mathrm{FG}}^{+} 17\right.$ & $?$ & \\
\hline Full substar & 1 & Section 3.3, $\left[\mathrm{CFG}^{+} 17\right.$ & $?$ & \\
\hline Dually chordal & 1 & [JKLW16] & 1 & [JKLW16] \\
\hline Cograph & 1 & [JKLW16] & $?$ & \\
\hline Split & 1 & [KP90], [JKLW16] & 1 & [JKLW16], Section 4.1 \\
\hline
\end{tabular}

Table 6.1: A resume of the main results on transversals of longest paths and longest cycles. Cells with a question mark indicates that the corresponding class has not been studied yet.

Problem 6.1. Find better upper bounds for $\operatorname{lpt}(G)$ and $\operatorname{lct}(G)$ when $G$ is arbitrary.

Problem 6.2. Are there constant upper bounds for $\operatorname{lpt}(G)$ and $\operatorname{lct}(G)$ when $G$ is arbitrary?

Conjecture 6.3. If $G$ is a connected graph, then $\operatorname{lpt}(G) \leq 2$.

Conjecture 6.4. If $G$ is a 2-connected graph, then $\operatorname{lct}(G) \leq 2$.

Problem 6.5. Find better upper bounds for $\operatorname{lpt}(G)$ and $\operatorname{lct}(G)$ when $G$ is a partial k-tree.

Conjecture 6.6. If $G$ is a connected partial 3-tree, then $\operatorname{lpt}(G)=2$.

Conjecture 6.7. If $G$ is a 2-connected partial 4-tree, then $\operatorname{lct}(G)=2$.

Problem 6.8. Find a better upper bound for $\operatorname{lpt}(G)$ when $G$ is a partial 4-tree.

Problem 6.9. Find better upper bounds for $\operatorname{lpt}(G)$ and $\operatorname{lct}(G)$ when $G$ is chordal.

Conjecture 6.10. If $G$ is a connected chordal graph, then $\operatorname{lpt}(G)=1$.

Conjecture 6.11. If $G$ is a 2-connected chordal graph, then $\operatorname{lct}(G)=1$.

Problem 6.12. Find better upper bounds for $\operatorname{lpt}(G)$ and $\operatorname{lct}(G)$ when $G$ is a k-tree.

Conjecture 6.13. If $G$ is a $k$-tree, then $\operatorname{lpt}(G)=1$.

Conjecture 6.14. If $G$ is a 2-connected $k$-tree, then $\operatorname{lct}(G)=1$.

Conjecture 6.15. If $G$ is a 3-tree, then $\operatorname{lpt}(G)=1$.

Conjecture 6.16. If $G$ is a 2-connected bipartite permutation graph, then $\operatorname{lct}(G)=1$.

Conjecture 6.17. If $G$ is a 2-connected full substar graph, then $\operatorname{lct}(G)=1$. 


\begin{tabular}{|l|c|c|c|}
\hline Class & $\tau / \nu$ & References & Tight? \\
\hline Arbitrary & 2.87 & {$[$ Hax99] } & No \\
\hline Partial 6-tree & 2 & Section [5.2] [BFG18] & No \\
\hline 3-tree & 1.8 & Section [5.3 & No \\
\hline Planar & 2 & {$[$ Tuz90] } & Yes \\
\hline$K_{3,3}$-free & 2 & {$[$ Kri95] } & Yes \\
\hline No $K_{5}$-subdivision & 2 & {$[$ Pul15] } & Yes \\
\hline$K_{4}$-free planar & 1.5 & {$[$ HKT12a] } & Yes \\
\hline Planar triangulation & 1.5 & Section 5.4 & Yes \\
\hline Tripartite & 1.87 & {$[$ Sze16] } & No \\
\hline 4-partite & 2 & {$[$ ALBT11] } & Yes \\
\hline
\end{tabular}

Table 6.2: A resume of the main results on Tuza's Conjecture.

Problem 6.18. Find upper bounds for $\operatorname{lpt}(G)$ and $\operatorname{lct}(G)$ when $G$ is a substar graph.

Conjecture 6.19. If $G$ is a 2-connected cograph, then $\operatorname{lct}(G)=1$.

Problem 6.20. Find better upper bounds for $\operatorname{lpt}(G)$ and $\operatorname{lct}(G)$ when $G$ is planar.

Problem 6.21. Find better upper bounds for $\operatorname{lpt}(G)$ and $\operatorname{lct}(G)$ when $G$ is a polyhedral graph.

Problem 6.22. Find upper bounds for $\operatorname{lpt}(G)$ and $\operatorname{lct}(G)$ when $G$ is a biconvex graph.

Problem 6.23. Find upper bounds for $\operatorname{lpt}(G)$ and $\operatorname{lct}(G)$ when $G$ is a distance hereditary graph.

Problem 6.24. Find upper bounds for $\operatorname{lpt}(G)$ and $\operatorname{lct}(G)$ when $G$ is a permutation graph.

Problem 6.25. Find better upper bounds for $\operatorname{lpt}(G)$ and $\operatorname{let}(G)$ when $G$ is bipartite.

Problem 6.26. Find upper bounds for $\operatorname{lpt}(G)$ and $\operatorname{lct}(G)$ when $G$ is a comparability graph.

Problem 6.27. Find upper bounds for $\operatorname{lpt}(G)$ and $\operatorname{lct}(G)$ when $G$ is a circle graph.

Problem 6.28. Find upper bounds for $\operatorname{lpt}(G)$ and $\operatorname{lct}(G)$ when $G$ is a perfect graph.

\subsection{Triangles}

As in the case of longest paths and longest cycles, new results regarding triangles can be obtained for several classes of graphs. In Figure 6.2 and Table 6.2 we show maps for the classes that have been studied for Tuza's Conjecture. They show us good insights on what steps this research can be continued. We present them as open problems.

Problem 6.29. Answer Tuza's Conjecture.

Problem 6.30. Find a better upper bound for $\tau(G) / \nu(G)$ when $G$ is an arbitrary graph.

Problem 6.31. Find a better upper bound for $\tau(G) / \nu(G)$ when $G$ is a partial 6-tree. 


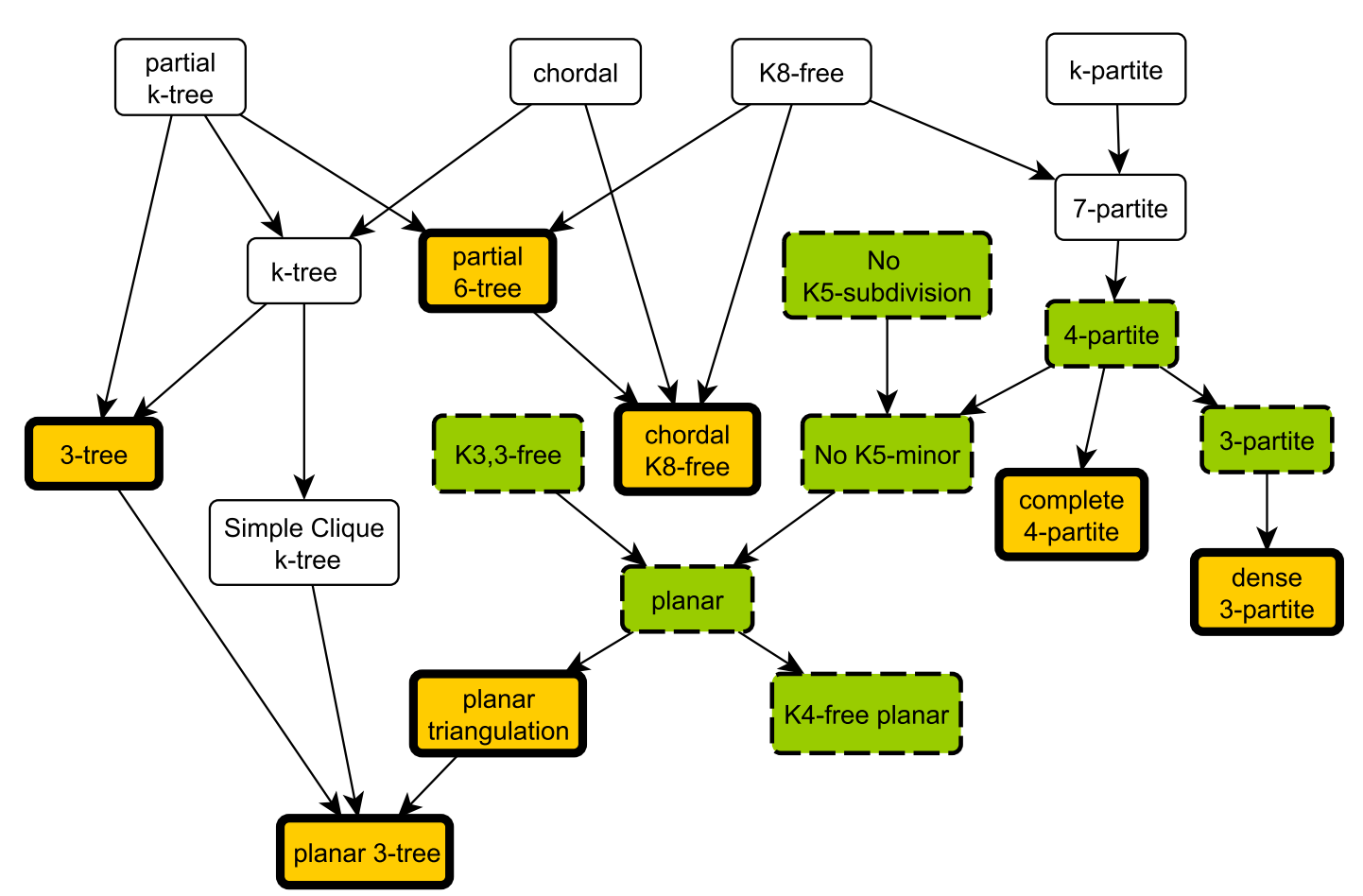

Figure 6.2: A map of the graph classes mentioned for Tuza's Conjecture. There are results for the classes indicated with a dashed line and they are due to other people. We have results for the classes indicated with a thick line. The classes indicated with a thin straight line have not yet been studied.

Problem 6.32. Find a better upper bound for $\tau(G) / \nu(G)$ when $G$ is a 3-tree.

Problem 6.33. Find a better upper bound for $\tau(G) / v(G)$ when $G$ is tripartite.

Problem 6.34. Find an upper bound for $\tau(G) / \nu(G)$ when $G$ is a chordal graph.

Problem 6.35. Find an upper bound for $\tau(G) / \nu(G)$ when $G$ is a partial $k$-tree.

Problem 6.36. Find an upper bound for $\tau(G) / \nu(G)$ when $G$ is a k-tree.

Problem 6.37. Find an upper bound for $\tau(G) / \nu(G)$ when $G$ is $K_{8}$-free.

Problem 6.38. Find an upper bound for $\tau(G) / \nu(G)$ when $G$ is 7-partite.

Problem 6.39. Find an upper bound for $\tau(G) / \nu(G)$ when $G$ is k-partite.

Problem 6.40. Characterize graphs for which $\tau(G)=2 v(G)$.

Problem 6.41. Characterize graphs for which $\tau(G)=\frac{3}{2} v(G)$.

Problem 6.42. Characterize graphs for which $\tau(G)=v(G)$. 


\subsection{Other Types of Transversals}

One can extend the question of transversal and packing of triangles to any $k$-clique. This extension was considered for the case $k=4$ by Szestopalow [Sze16, Chapter 5]. Moreover, Tuza asked a similar question when the graph considered is arbitrary and not bipartite [Tuz01, Problem 35]. It would be interesting to show new results in these directions.

Beside longest paths, longest cycles, and triangles, there are also problems regarding other types of transversals and packings that can be studied, both with algorithmic and structural approaches. We close this chapter mentioning some of the literature regarding them: transversals of odd cycles [FHRV07, Tho88, RR01, Yan78, RSV04, Huf09], transversals of maximal cliques [EGT92, CFT93, CCCY96, GR00, LS15, BNR96, CCCY96, BDLS06, LC06, GLS02, AST91, BT09, SCK08], and Woodall's conjecture [FY87, LW01, LW06]. 


\section{Bibliography}

[ALBT11] S. Aparna Lakshmanan, C. Bujtás, and Z. Tuza. Small edge sets meeting all triangles of a graph. Graphs and Combinatorics, 28(3):381-392, 2011.

[ALBT16] S. Aparna Lakshmanan, C. Bujtás, and Z. Tuza. Induced cycles in triangle graphs. Discrete Applied Mathematics, 209:264-275, 2016.

[APC90] S. Arnborg, A. Proskurowski, and D. G. Corneil. Forbidden minors characterization of partial 3-trees. Discrete Mathematics, 80(1):1-19, 1990.

[AST90] N. Alon, P. Seymour, and R. Thomas. A separator theorem for nonplanar graphs. J Am Math Soc, 3:801-808, 1990.

[AST91] T. Andreae, M. Schughart, and Z. Tuza. Clique-transversal sets of line graphs and complements of line graphs. Discrete Mathematics, 88(1):11-20, 1991.

[BDLS06] F. Bonomo, G. Durán, M. C. Lin, and J. L. Szwarcfiter. On balanced graphs. Mathematical Programming, 105(2-3):233-250, 2006.

[BFG18] F. Botler, C. G. Fernandes, and J. Gutiérrez. On tuza's conjecture for graphs with treewidth at most 6 . In 3 Encontro de Teoria da Computação (ETC), volume 3, Porto Alegre, RS, Brasil, 2018. SBC.

[BGLS04] P. N. Balister, E. Győri, J. Lehel, and R. H. Schelp. Longest paths in circular arc graphs. Combin. Probab. Comput., 13(3):311-317, 2004.

[BM08] J. A. Bondy and U. S. R. Murty. Graph theory, volume 244 of Graduate Texts in Mathematics. Springer, New York, 2008.

[BNR96] V. Balachandran, P. Nagavamsi, and C. P. Rangan. Clique transversal and clique independence on comparability graphs. Information Processing Letters, 58(4):181-184, May 1996.

[Bod98] H. L. Bodlaender. A partial $k$-arboretum of graphs with bounded treewidth. Theoretical Computer Science, 209(1):1-45, 1998.

[Bol78] B. Bollobás. Extremal graph theory. Academic Press, 1978.

[Bro41] R. L. Brooks. On colouring the nodes of a network. Mathematical Proceedings of the Cambridge Philosophical Society, 37(2):194-197, 1941.

[BT09] G. Bacsó and Z. Tuza. Clique-transversal sets and weak 2-colorings in graphs of small maximum degree. Discrete Mathematics $\mathcal{E}$ Theoretical Computer Science, 11(2):15-24, 2009. 
[CCCY96] M. S. Chang, Y. H. Chen, G. J. Chang, and J. H. Yan. Algorithmic aspects of the generalized clique-transversal problem on chordal graphs. Discrete Applied Mathematics, 66(3):189-203, 1996.

$\left[\mathrm{CDM}^{+} 14\right]$ G. Chapuy, M. DeVos, J. McDonald, B. Mohar, and D. Scheide. Packing triangles in weighted graphs. SIAM Journal on Discrete Mathematics, 28(1):226-239, 2014.

[CEF $\left.{ }^{+} 17\right]$ G. Chen, J. Ehrenmüller, C. G. Fernandes, C. G. Heise, S. Shan, P. Yang, and A. N. Yates. Nonempty intersection of longest paths in series-parallel graphs. Discrete Mathematics, 340(3):287-304, 2017.

[CFG98] G. Chen, R. J. Faudree, and R. J. Gould. Intersections of longest cycles in $k$-connected graphs. Journal of Combinatorial Theory, Series B, 72(1):143 - 149, 1998.

[CFG $\left.{ }^{+} 17\right]$ M. R. Cerioli, C. G. Fernandes, R. Gómez, J. Gutiérrez, and P. Lima. Transversals of longest paths. Electronic Notes in Discrete Mathematics, 62(Supplement C):135 - 140, 2017. LAGOS'17 - IX Latin and American Algorithms, Graphs and Optimization.

[CFT93] G. J. Chang, M. Farber, and Z. Tuza. Algorithmic aspects of neighbourhood numbers. SIAM J. Discrete Math., 6(1):24-29, 1993.

[Che15] F. Chen. Nonempty intersection of longest paths in a graph with a small matching number. Czechoslovak Mathematical Journal, 65(140):545-553, 2015.

[CHM09] Q. Cui, P. Haxell, and W. Ma. Packing and covering triangles in planar graphs. Graphs and Combinatorics, 25(6):817-824, 2009.

[CL16] M. R. Cerioli and P. Lima. Intersection of longest paths in graph classes. Electronic Notes in Discrete Mathematics, 55:139-142, 2016.

[CW16] D. W. Cranston and D. B. West. An introduction to the discharging method via graph coloring. https://arxiv.org/abs/1306.4434, 2016.

[DF55] G. Dantzig and D. Fulkerson. On the max-flow min-cut theorem of networks. Inequalities and Related Systems, 38:215-222, 1955.

[dFMW13] S. F. de Rezende, C. G. Fernandes, D. M. Martin, and Y. Wakabayashi. Intersecting longest paths. Discrete Mathematics, 313:1401-1408, 2013.

[Die10] R. Diestel. Graph Theory, 4th Edition, volume 173 of Graduate texts in mathematics. Springer, 2010.

[EGT92] P. Erdôs, T. Gallai, and Z. Tuza. Covering the cliques of a graph with vertices. Discrete Mathematics, 108(1-3):279-289, 1992.

[EK68] P. Erdős and G. Katona, editors. Theory of Graphs. Proceedings of the Colloquium held at Tihany, Hungary, September 1966. Academic Press, New York, 1968. Problem 4 (T. Gallai), p. 362. 
[FF56] L. R. Ford and D. R. Fulkerson. Maximal Flow through a Network. Canadian Journal of Mathematics, 8:399-404, 1956.

[FG17] C. G. Fernandes and J. Gutiérrez. Hitting all longest cycles in a graph. In Anais do XXXVII Congresso da Sociedade Brasileira de Computação, pages 87-90, 2017.

[FHRV07] S. Fiorini, N. Hardy, B. A. Reed, and A. Vetta. Approximate min-max relations for odd cycles in planar graphs. Mathematical Programming, 110(1):7191, 2007.

[Fis89] D. C. Fisher. Lower bounds on the number of triangles in a graph. Journal of Graph Theory, 13(4):505-512, 1989.

[FT06] F. V. Fomin and D. M. Thilikos. New upper bounds on the decomposability of planar graphs. Journal of Graph Theory, 51(1):53-81, 2006.

[FY87] P. Feofiloff and D. H. Younger. Directed cut transversal packing for sourcesink connected graphs. Combinatorica, 7(3):255-263, 1987.

[Gav74] F. Gavril. The intersection graphs of subtrees in trees are exactly the chordal graphs. Journal of Combinatorial Theory, Series B, 16(1):47-56, 1974.

[GLS02] D. Guillermo, M. C. Lin, and J. L. Szwarcfiter. On clique-transversals and clique-independent sets. Annals of Operations Research, 116(1-4):71-77, 2002.

[Gol04] M. C. Golumbic. Algorithmic Graph Theory and Perfect Graphs (Annals of Discrete Mathematics, Vol 57). North-Holland Publishing Co., Amsterdam, The Netherlands, 2004.

[GR00] V. Guruswami and C. P. Rangan. Algorithmic aspects of clique-transversal and clique-independent sets. Discrete Applied Mathematics, 100(3):183-202, 2000.

[Gro14] J. L. Gross. Embeddings of graphs of fixed treewidth and bounded degree. ARS Mathematica Contemporanea, 7:379-403, 2014.

[GS16] G. Golan and S. Shan. Nonempty intersection of longest paths in $2 K_{2}$-free graphs. Available as arXiv:1611.05967, 2016.

[GT87] E. Győri and Z. Tuza. Decompositions of graphs into complete subgraphs of given order. Studia scientiarum mathematicarum Hungarica, 22:315 - 320, 1987.

[Gut18] J. Gutiérrez. Transversals of longest cycles in chordal and bounded treewidth graphs. In LATIN 2018: Theoretical Informatics - 13th Latin American Symposium, Buenos Aires, Argentina, April 16-19, 2018, Proceedings, pages 558-571, 2018.

[Hax99] P. E. Haxell. Packing and covering triangles in graphs. Discrete Mathematics, 195(1):251-254, 1999. 
[Hei13] M. Heinz. Tree-decomposition: Graph minor theory and algorithmic implications. Master's thesis, Technischen Universität Wien, 2013.

[Hel23] E. Helly. Über Mengen konvexer Körper mit gemeinschaftlichen Punkte. Jahresbericht der Deutschen Mathematiker-Vereinigung, 32:175-176, 1923.

[Hip08] T. Hippchen. Intersections of Longest Paths and Cycles. PhD thesis, Georgia State University, 2008.

[HK98] P. E. Haxell and Y. Kohayakawa. Packing and covering triangles in tripartite graphs. Graphs and Combinatorics, 14(1):1-10, 1998.

[HKT12a] P. E. Haxell, A. Kostochka, and S. Thomassé. Packing and covering triangles in $K_{4}$-free planar graphs. Graphs and Combinatorics, 28(5):653-662, 2012.

[HKT12b] P. E. Haxell, A. Kostochka, and S. Thomassé. A stability theorem on fractional covering of triangles by edges. European Journal of Combinatorics, 33(5):799-806, 2012.

[Hor72] W. A. Horn. Three results for trees, using mathematical induction. Journal of Research of the National Bureau af Standards - B. Mathematical Sciences, 76B:39-43, 1972.

[Huf09] F. Huffner. Algorithm engineering for optimal graph bipartization. J. Graph Algorithms Appl., 13(2):77-98, 2009.

[JKLW16] A. S. Jobson, A. E. Kézdy, J. Lehel, and S. C. White. Detour trees. Discrete Applied Mathematics, 206:73-80, 2016.

[Joo14] F. Joos. A characterization of substar graphs. Discrete Applied Mathematics, 175:115-118, 2014.

[Joo15] F. Joos. A note on longest paths in circular arc graph. Discussiones Mathematicae Graph Theory, 35(3):419-426, 2015.

[JS97] S. Jendrol and Z. Skupień. Exact numbers of longest cycles with empty intersection. European Journal of Combinatorics, 18(5):575 - 578, 1997.

[Kö16] D. König. Über Graphen und ihre Anwendung auf Determinantentheorie und Mengenlehre. Mathematische Annalen, 77:453-465, 1916.

[KP90] S. Klavžar and M. Petkovšek. Graphs with nonempty intersection of longest paths. Ars Combinatoria, 29:43-52, 1990.

[Kri95] M. Krivelevich. On a conjecture of Tuza about packing and covering of triangles. Discrete Mathematics, 142(1-3):281-286, 1995.

[LC06] C. Lee and M. S. Chang. Distance-hereditary graphs are clique-perfect. Discrete Applied Mathematics, 154(3):525-536, 2006.

[Lim16] P. T. Lima. Interseção de caminhos mais longos em grafos. Master's thesis, Rio de Janeiro: UFRJ/COPPE, 2016. 
[LS15] Z. Liang and E. Shan. The clique-transversal set problem in claw-free graphs with degree at most 4. Information Processing Letters, 115(2):331-335, 2015.

[LW01] O. Lee and Y. Wakabayashi. Note on a min-max conjecture of woodall. Journal of Graph Theory, 38(1):36-41, 2001.

[LW06] O. Lee and A. Williams. Packing dicycle covers in planar graphs with no $k_{5}-e$ minor. In LATIN, 2006.

[Pet91] J. Petersen. Die theorie der regulären graphs. Acta Math., 15:193-220, 1891.

[Pul15] G. J. Puleo. Tuza's conjecture for graphs with maximum average degree less than 7. European Journal of Combinatorics, 49:134-152, 2015.

[RR01] D. Rautenbach and B. A. Reed. The erdos-pósa property for odd cycles in highly connected graphs. Combinatorica, 21(2):267-278, 2001.

[RS14] D. Rautenbach and J.-S. Sereni. Transversals of longest paths and cycles. SIAM J. Discrete Math., 28(1):335-341, 2014.

[RSV04] B. A. Reed, K. Smith, and A. Vetta. Finding odd cycle transversals. Operations Research Letters, 32(4):299-301, 2004.

[SCK08] E. Shan, T. C. E. Cheng, and L. Kang. Bounds on the clique-transversal number of regular graphs. Science in China Series A: Mathematics, 51(5):851863, 2008.

[ST95] I. A. Stewart and B. Thompson. On the intersections of longest cycles in a graph. Experiment Mathematics, 4(1):41 - 48, 1995.

[Sze16] Szestopalow, M. Matchings and Covers in Hypergraphs. PhD thesis, 2016.

[SZZ13] A. Shabbir, C. T. Zamfirescu, and T. I. Zamfirescu. Intersecting longest paths and longest cycles: a survey. Electronic Journal of Graph Theory and Applications, 1:56-76, 2013.

[Tho78] C. Thomassen. Hypohamiltonian graphs and digraphs. In Theory and Applications of Graphs: Proceedings, Michigan May 11-15, 1976, volume 642, pages 557-571. Springer, Berlin, Heidelberg, 1978.

[Tho88] C. Thomassen. On the presence of disjoint subgraphs of a specified type. Journal of Graph Theory, 12(1):101-111, 1988.

[Tut56] W. T. Tutte. A theorem on planar graphs. Transactions of the American Mathematical Society, 82:99-116, 1956.

[Tuz81] Z. Tuza. Conjecture, finite and infinite sets. In Proceedings of the Colloquia Mathematica Societatis Janos Bolyai, Eger, Hungary, North-Holland, Amsterdam, page $888,1981$.

[Tuz90] Z. Tuza. A conjecture on triangles of graphs. Graphs and Combinatorics, 6(4):373-380, 1990. 
[Tuz01] Z. Tuza. Unsolved combinatorial problems Part 1. Basic Research in Computer Science (BRICS), 1, 2001.

$\left[\mathrm{vABD}^{+} 15\right]$ S. A. van Aardt, A. P. Burger, J. E. Dunbar, M. Frick, B. Llano, C. Thomassen, and R. Zuazua. Destroying longest cycles in graphs and digraphs. Discrete Applied Mathematics, 186(Supplement C):251-259, 2015.

[Viz89] V. G. Vizing. On an estimate of the chromatic class of a p-graph. Diskret. Analiz., 3:25-30, 1989.

[Wag37] K. Wagner. Über eine eigenschaft der ebenen komplexe. Mathematische Annalen, 114:570-590, 1937.

[WV74] H. Walther and H.-J. Voss. Über Kreise in Graphen. VEB Deutscher Verlag der Wissenschaften, 1974.

[Yan78] M. Yannakakis. Node-and edge-deletion NP-complete problems. In Proceedings of the Tenth Annual ACM Symposium on Theory of Computing (STOC), pages 253-264, New York, NY, USA, 1978. ACM.

[Zam76] T. Zamfirescu. On longest paths and circuits in graphs. Math. Scand., 38(2):211-239, 1976. 


\section{Index}

$\left(K_{4}\right.$, gem $)$-free graph, 4

lct, 3

lpt, 3

$\simeq, 8$

$\tau, 3$

$k$-attractor, 15

$k$-clique, 9

$k$-cross, 14

$k$-fenced, 14

$k$-intersects, 14

$k$-partite graph, 10

$k$-tree, 12

$t$-inside cycle, 57

$t$-inside path, 57

$t$-inside vertex, 56

$t$-outside cycle, 57

$t$-outside path, 57

$t$-outside vertex, 56

2-connected graph, 9

adjacent, 7,8

ancestor, 83

arc, 10

attractor, 15

bag, 11

bipartite graph, 10

bipartite permutation graph, 33

bramble, 18

branch, 15

break, 14

breaking vertex, 14

bridge, 10

center of a star, 11

chordal graph, 13

clique, 9

clique tree, 13

complete $k$-partite graph, 10 complete graph, 8

connected graph, 9

cross, 14

cycle, 8

degree, 7

descendant, 83

digraph, 10

distance in $X, 37$

distance in $Y, 37$

dual graph, 101

edge, 7

edge-coloring, 8

end, 7

equivalent, 14

extreme, 8

face, 101

fence, 14

full substar graph, 30

full tree decomposition, 11

graph, 7

height, 83

hit, 37

independent set, 8

induced graph, 8

inside vertex, 57

internal vertex, 8

intersection model, 30

irreducible, 85

joined vertices, 8

jump, 57

leaf, 10

left-most path, 38 
line representatión, 33

longest cycle, 14

longest cycle transversal, 3

longest path, 14

longest path transversal, 3

matching, 8

maximum average degree, 4

neighbor, 7

node of a digraph, 10

node of a tree, 11,30

odd-wheel-free graph, 4

order of a bramble, 19

outside vertex, 57

packing, 1,83

parallel edges, 37

parent, 83

partial $k$-tree, 12

partial orientation, 10

parts of a cycle, 14

path, 8

planar embedding, 101

planar graph, 101

planar triangulation, 101

reducible, 85

representative, 84

robust, 85

rooted tree, 83

rooted tree decomposition, 83

split graph, 41

spliting of a graph, 41

star, 11

subgraph, 8

successor, 83

tails of a path, 13

transversal, 1, 83

tree, 10

tree decomposition, 11

treewidth, 11

triangle, 9

triangle packing, 3

triangle transversal, 3

triangle-3-colorable graph, 4 tripartite graph, 11

vertex, 7

width, 11 